\title{
Innovationsland Österreich F\&E, Unternehmensentwicklung und Standortattraktivität
}

\author{
Christian KEUSCHNIGG \\ Universität St. Gallen, FGN-HSG, und Wirtschaftspolitisches Zentrum Wien \\ Christian.Keuschnigg@unisg.ch \\ Brigitte ECKER und Sascha SARDADVAR \\ WPZ Research GmbH, Wien \\ Christian REINER \\ Lauder Business School Vienna und WPZ Research GmbH, Wien
}

6. Juni 2017

\begin{abstract}
Das Innovationssystem hat wesentlichen Einfluss auf die Entwicklung und die internationale Wettbewerbsfähigkeit der Unternehmen. F\&E-intensive Unternehmen wachsen schneller, schaffen mehr Arbeitsplätze und sind krisenrobuster. Sie stärken das Wachstum und stabilisieren die Konjunktur. Der Staat ist auf drei Ebenen gefordert: (i) Mit den Investitionen in die Grundlagenforschung, die forschungsnahe Ausbildung und die technologische Infrastruktur erbringt er zentrale Vorleistungen für die private F\&E. (ii) Mit dem Abbau von Marktstörungen durch Schutz intellektueller Eigentumsrechte, Beseitigung von Finanzierungsengpässen und mit günstigen rechtlichen und steuerlichen Rahmenbedingungen verbessert er Umfang und Qualität der privaten F\&E. (iii) Mit komplementären Maßnahmen, die den Strukturwandel erleichtern und die Standortattraktivität wahren, stärkt er die günstigen Auswirkungen der privaten Innovation auf die gesamtwirtschaftliche Entwicklung. In zehn Punkten fasst die Studie die Prioritäten für eine neue Innovationsstrategie zusammen.
\end{abstract}

\footnotetext{
* Wir danken dem Rat für Forschung und Technologieentwicklung für Auftrag und Finanzierung. Bei Brigitte Tschudi, Hannah Winterberg und David Bader bedanken wir uns für die wertvolle Forschungsassistenz.

WPZ - Wirtschaftspolitisches Zentrum WPZ - Wirtschaftspolitisches Zentrum - St. Gallen

Forschungsgemeinschaft für Nationalökonomie (FGN-HSG)
Universität St. Gallen, Varnbüelstrasse 19, CH-9000 St. Galle

Forschungsgemeinschaft für Nationalökonomie (FGN-HSG)
Universität St. Gallen, Varnbüelstrasse 19, CH-9000 St. Gallen

www.fgn.unisg.ch/wpz; office@wpz-fgn.com

WPZ - Wirtschaftspolitisches Zentrum - Wien c/o Konrad \& Partner

Rotenturmstrasse 13, A-1010 Wien www.wpz-fgn.com; office@wpz-fgn.com Tel.: +4369910494150
} 


\section{Inhalt}

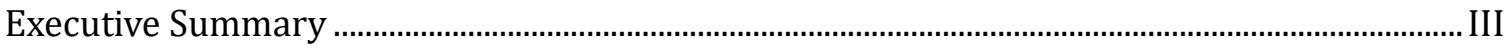

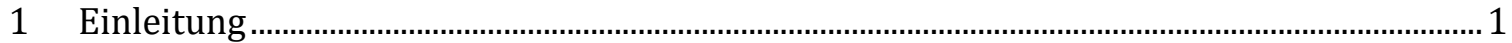

2 Innovationsland Österreich: Ein statistisches Porträt............................................................... 4

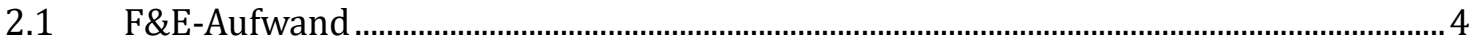

2.1.1 Entwicklung der Forschungsquoten............................................................................ 4

2.1.2 Österreichs Position in der Grundlagenforschung..........................................................

2.1.3 F\&E nach Betriebsgrößen und Sektoren..........................................................................

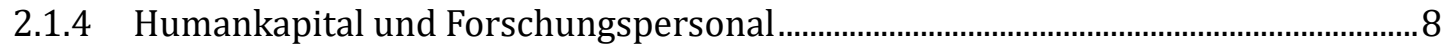

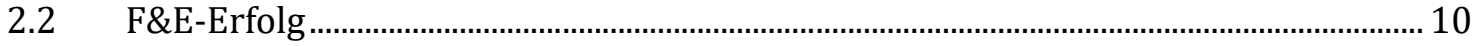

2.2.1 Österreich in internationalen Rankings .......................................................................10

2.2.2 Patentierungen im internationalen Vergleich............................................................ 12

2.2.3 Prozessinnovationen und Produktivität nach Branchen..............................................113

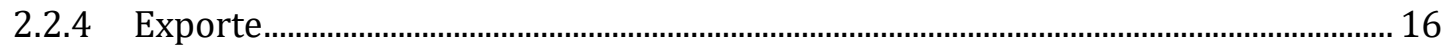

2.2.5 Wissenschaftliche Publikationen .......................................................................................16

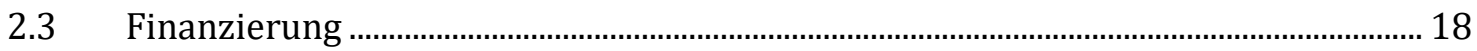

2.3.1 Staatliche und privatwirtschaftliche Finanzierung .................................................... 18

2.3.2 Drittmittelfinanzierung an den Universitäten...............................................................2.

2.3.3 Finanzierungsbeitrag ausländischer Unternehmen....................................................... 22

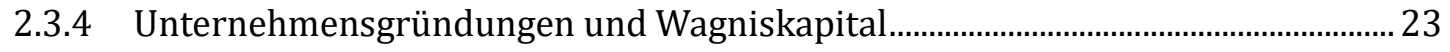

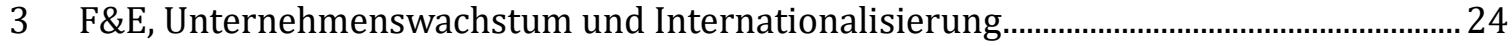

3.1 Produktivitätsentwicklung in der Industrie.....................................................................25

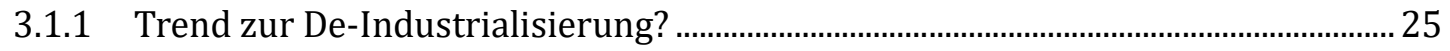

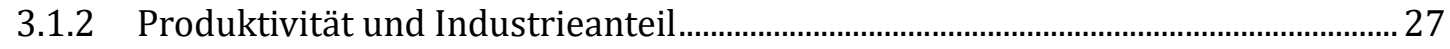

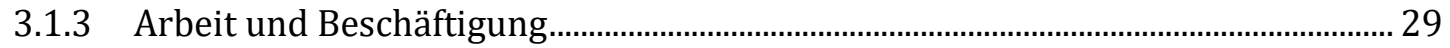

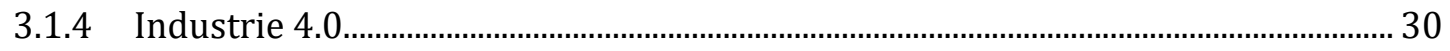

3.2 F\&E und Unternehmenswachstum ............................................................................... 32

3.2.1 F\&E, Investition und Beschäftigung.......................................................................33

3.2.2 Unternehmenswachstum im Branchen- und Ländervergleich....................................36

3.2.3 Heterogene F\&E-Effekte auf das Unternehmenswachstum........................................... 39

3.2.4 Wachstumshindernisse.................................................................................................. 41

3.2.5 Fördert Innovation die Krisenrobustheit? ................................................................... 42

3.3 F\&E und Internationalisierung der Unternehmen ........................................................... 46

3.3.1 F\&E, Produktivität und Exporte ....................................................................................... 46

3.3.2 Produktivitäts- und Exportentwicklung in Österreich............................................... 49

3.3.3 F\&E und ausländische Direktinvestitionen ...................................................................52

3.3.4 Determinanten der F\&E-Standortwahl......................................................................... 55

3.3.5 Räumliche Koppelung von F\&E und Produktion.........................................................57

3.3.6 Rückkehr der Industrie aus dem Ausland .......................................................................58

4 Wirtschaftspolitische Rahmenbedingungen .............................................................................60

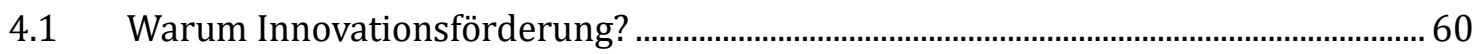

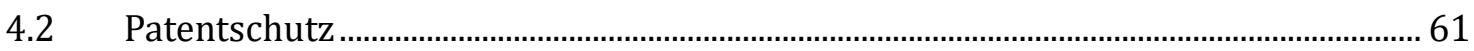

4.3 Grundlagenforschung ....................................................................................................... 64 


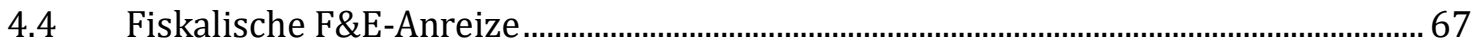

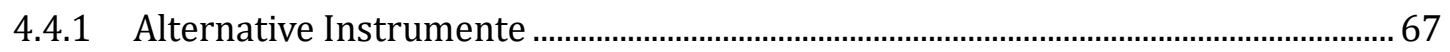

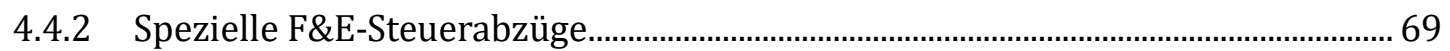

4.4.3 Begünstigung von F\&E-Erträgen in Patentboxen ........................................................ 71

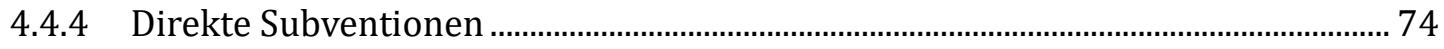

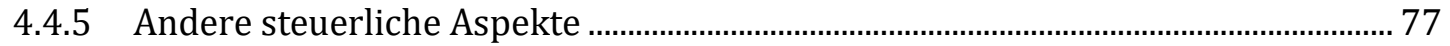

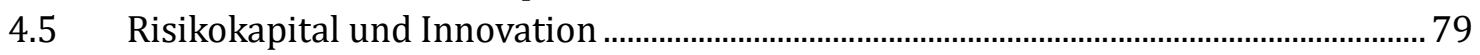

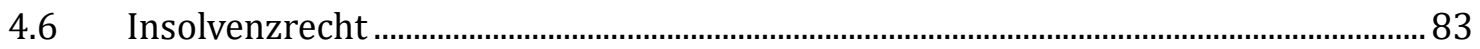

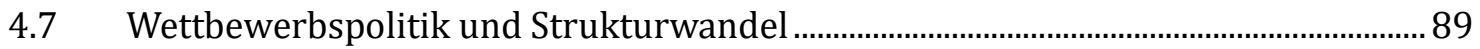

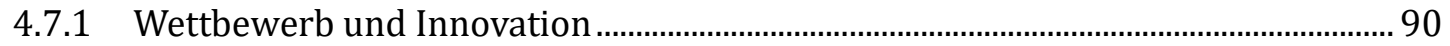

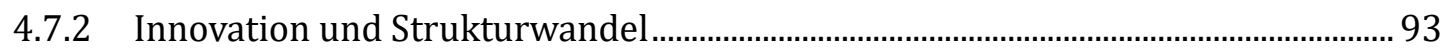

5 Zentrale Elemente einer neuen Innovationsstrategie ........................................................... 101

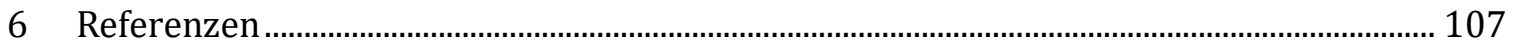

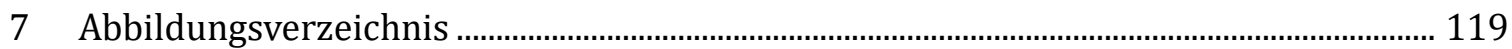

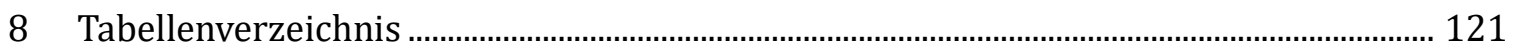




\section{Executive Summary}

Innovation treibt das Wachstum der Unternehmen und der Volkswirtschaft. Österreich hat in den letzten 20 Jahren bei den F\&E-Investitionen aufgeholt. Der Anteil der F\&E-Investitionen am BIP lag Mitte der 1990er-Jahre weit hinter den führenden Industriestaaten und hat inzwischen die meisten überholt. Damit übertrifft Österreich bereits heute das von der EU für 2020 anvisierte Ziel eines F\&E-Anteils am BIP von 3\%. Das Volumen der F\&E-Investitionen hat sich seit 1995 mehr als verdoppelt. Eine wichtige Rolle in diesem Aufhol- und Überholprozess spielt der industrielle Sektor. Die Betriebe des Verarbeitenden Gewerbes geben knapp 9\% der Bruttowertschöpfung für F\&E-Investitionen aus. Mit 62\% entfällt der überwiegende Anteil der privaten F\&E-Investitionen auf das Verarbeitende Gewerbe. Dennoch liegt die F\&E-Intensität deutlich niedriger als in anderen, vergleichbaren EU-Staaten wie Finnland oder Schweden. Auch wenn in den mittleren Technologiesegmenten die Produktivität bemerkenswert hoch und das Produktivitätswachstum gut ist, hat es Österreich bisher noch nicht geschafft, zu den führenden Innovationsländern wie Schweiz, Dänemark, Deutschland, Finnland, die Niederlande und Schweden aufzuschließen. Umso wichtiger ist es, die Wertschöpfungskette der Innovation vom Beginn bis zum Ende systematisch auf Stärken und Schwächen zu überprüfen und einen systemischen Politikansatz zu entwickeln.

Am Beginn stehen die Grundlagenforschung und die universitäre Ausbildung an der Grenze der Forschung. Diese erbringen Vorleistungen für die private F\&E wie neue Anwendungen, Patente und hoch qualifiziertes Forschungspersonal. Die empirische Forschung schätzt, dass je nach Branche die privaten F\&E-Ausgaben um 7\% steigen, wenn die Ausgaben für Grundlagenforschung um 10\% wachsen. Private F\&E treibt das Unternehmenswachstum. Mit Produkt- und Prozessinnovationen erringen die Unternehmen einen Qualitätsvorsprung und Kostenvorteile, welche Investitionen und Beschäftigung erst rentabel machen.

Für stark wachsende Firmen wird der Heimmarkt rasch zu klein. Spezialisierte und innovative Firmen müssen den Weltmarkt erschließen, um ihr Wachstumspotenzial auszuschöpfen. Dort treffen sie auf weltweite Konkurrenz und können sich nur mit überlegener Innovation behaupten. Je größer die Exportfirmen und je wichtiger die Auslandsmärkte werden, desto eher entwickeln sie sich zu großen Konzernen, die in vielen Ländern aktiv sind. Multinationale Unternehmen sind die produktivsten und forschungsintensivsten. Gerade wegen ihrer überdurchschnittlichen F\&E-Intensität und anhaltenden Innovationsfähigkeit sind sie so groß und produktiv geworden. Wegen ihrer Größe sind sie am ehesten selbst in der Grundlagenforschung aktiv und suchen häufig die Kooperation mit Universitäten und Zentren der Grundlagenforschung. Die empirische Forschung schätzt, dass Exportfirmen etwa 40\% und multinationale Unternehmen um bis zu $55 \%$ produktiver sind als rein national orientierte Firmen.

Weil sie mit Exporten ausländische Märkte bearbeiten, sind innovative Firmen überaus mobil und können leicht den Standort verlegen, um direkt am Absatzort zu produzieren. Die Standortattraktivität bestimmt, wie viel an Investition und Beschäftigung im Inland oder im Ausland stattfindet. Sie ist für die produktivsten und innovativsten Unternehmen mit dem größten Wachstumspotenzial besonders wichtig. Für die Beschäftigung ist entscheidend, dass Unternehmen nicht nur die F\&E in Österreich durchführen, sondern der Standort auch für die industrielle Produktion attraktiv bleibt. Der Trend zur digitalen, webbasierten 
Wirtschaft macht es leichter, die Wertschöpfungskette weltweit zu verteilen, um Kosten zu sparen oder andere Standortvorteile zu nutzen. Dabei ist die Standortattraktivität nicht nur für die Unternehmen als Ganzes, sondern für einzelne Funktionen wie F\&E, Produktion und Vertrieb sowie Finanzierung und Konzernführung am Hauptsitz separat zu überprüfen. Die Standortfrage stellt sich in allen Phasen der Innovationskette. Auch Universitäten müssen weltweit um die Starforscher und größten Studierendentalente konkurrieren.

Digitalisierung und Automatisierung von standardisierten Arbeitsschritten lösen einen kräftigen Strukturwandel aus und fordern sowohl Unternehmen als auch Bildungs- und Forschungspolitik heraus. Viele berufliche Tätigkeiten werden sich stark verändern. Neue und bessere Qualifikationen werden notwendig. Unternehmen werden mit großem F\&EAufwand neue Geschäftsmodelle entwickeln und traditionelle Produktionsmodelle ersetzen. Je mehr und je grundlegender die Innovationen sind, desto stärker ist der Strukturwandel, der sowohl innerhalb etablierter Unternehmen wie auch zwischen den Unternehmen durch Marktzutritt und Austritt erfolgt. Große Konzerne müssen ihren Produktzyklus erneuern, Produktion und Beschäftigung umstellen und mit ständiger Innovation ihre Marktstellung behaupten und ausbauen. Der Unternehmenssektor als Ganzes entwickelt sich durch Marktaustritt und Neugründungen weiter. Kapital und Arbeit werden neu eingesetzt. Die Produktivität kann nur dann steigen, wenn Kapital und Arbeit gezielt in die ertragreicheren Aktivitäten fließen. Innovationen können sich erst durchsetzen und nur dann mehr Einkommen schaffen, wenn der Strukturwandel gelingt und die Märkte erfolgreich Kapital und Arbeit von schrumpfenden auf wachsende Branchen und Unternehmen hinlenken.

Je weiter ein Land technologisch voranschreitet, umso eher fällt ihm die Rolle zu, selbst die Technologiegrenze zu verschieben, anstatt vorhandene Technologien zu verbessern. Zwar ist der Anteil der Grundlagenforschung an den gesamten F\&E-Investitionen gestiegen, dennoch liegt dieser Anteil am BIP weit hinter führenden Ländern wie Japan oder der Schweiz zurück. Ein Grund ist das Fehlen einer größeren Anzahl von multinationalen Konzernen, die am ehesten in die Grundlagenforschung investieren. Aber auch im universitären Bereich steckt bezüglich Lehrbelastung, Ausstattung mit Forschungsmitteln und Attraktivität für international führende ForscherInnen und Top-Talente ein erheblicher Investitionsbedarf.

Die wohl größte Herausforderung Österreichs besteht darin, dass die Unternehmen radikalere Innovationsstrategien verfolgen, neue Produkte und Prozesse mit größerem technologischem Vorsprung entwickeln und sich damit auf dem Weltmarkt eine dominantere Position sichern. Oft sind es gerade Start-Ups, die nicht von den Erfahrungen mit vorhandenen Produkten und Prozessen geprägt sind und eher ganz neue Lösungen ausprobieren. Für Start-Ups ist es hierzulande schwieriger als anderswo, privates Wagniskapital und damit Finanzierung und strategische Beratung aus einer Hand zu erhalten. Aktive Wagniskapitalgeber drängen ihre Unternehmen zu radikaleren Innovationsstrategien und trimmen sie auf Wachstumskurs. In der Intensivierung der Grundlagenforschung an den Universitäten und der Bereitstellung von Risiko- und Wagniskapital für innovative Unternehmen liegt das größte Potenzial zur weiteren Verbesserung.

Eine neue Innovationsstrategie für den Aufstieg zum Innovation Leader muss die gesamte Wertschöpfungskette der Innovation berücksichtigen, von der Grundlagenforschung über die private F\&E bis hin zur Kommerzialisierung in der Produktion. Sie soll eine klare Aufgabenteilung zwischen Staat und Privatwirtschaft ermöglichen, wonach der Staat 
wichtige Vorleistungen für die private Innovation erbringt und mit fiskalischen und regulatorischen Instrumenten Marktstörungen abbaut, damit der Wettbewerb das bestmögliche Ergebnis erzielen kann. Sie soll die Heterogenität der Firmen berücksichtigen. Junge innovative Unternehmen leiden häufig an Finanzierungsproblemen wie Mangel an Risikokapital, welcher die rasche Expansion drosselt. Große Konzerne, die den größten Teil der privaten F\&E-Ausgaben verantworten, haben dagegen wenig Mühe, Finanzierung aufzutreiben. Sie sind an vielen Standorten weltweit präsent und international mobil. Bei ihnen hat die Standortattraktivität Priorität. Damit ist die Politik gefordert, die zunehmende F\&E-Förderung im Ausland wie auch andere Standortnachteile wie z.B. den kleinen Binnenmarkt wenigstens teilweise zu kompensieren.

Die Innovationsstrategie soll einen systemischen Ansatz verfolgen und die Komplementaritäten zwischen den verschiedenen Politikfeldern ausnutzen. Allzu oft wird der Fokus zu eng auf die F\&E-Förderung gelegt. Diese kann jedoch einen kleinen oder großen volkswirtschaftlichen Ertrag haben, je nachdem ob an anderen Stellen Engpässe in der Wertschöpfungskette der Innovation bestehen. Wenn es an Innovationsfinanzierung mangelt und die Grundlagenforschung vernachlässigt wird, dann kann auch eine noch so großzügige F\&E-Förderung nur beschränkte Wirkung entfalten. Nur wenn ein Politikelement die Wirksamkeit anderer Maßnahmen positiv beeinflusst, also die Elemente komplementär zueinander sind, wird das Ganze mehr als die Summe der Teile. Die folgende Innovationsstrategie in zehn Punkten ist daher als Gesamtkonzept zu verstehen.

(1) Grundlagenforschung ausbauen. Die Bedeutung der Grundlagenforschung im Innovationssystem steigt, je mehr sich ein Land der Technologiegrenze annähert. Sie erbringt zentrale Vorleistungen für die private Innovation, indem sie F\&E-Personal an der Grenze der Forschung ausbildet und selbst neues, allgemein zugängliches Wissen schafft, das für die Kommerzialisierung verfügbar ist. Der Ausbau der Grundlagenforschung soll zudem effektive Anreize für den Technologietransfer in die Privatwirtschaft setzen.

(2) Patentierung unterstützen. Für Erfindungen mit großem wirtschaftlichem Potenzial ist eine Patentierung die vielleicht wichtigste Vorkehrung für einen wirksamen Schutz der Eigentumsrechte, damit mehr Investitionssicherheit entsteht und große F\&E-Investitionen sich besser rechnen.

(3) Gründungsdynamik stärken. Große Konzerne finanzieren zwar den größten Teil der privaten F\&E-Ausgaben, kleine Firmen sind jedoch pro Euro an F\&E-Ausgaben effektiver. Aktives Unternehmertum braucht eine Wertschätzung der Risikobereitschaft und nicht eine Stigmatisierung des Scheiterns. Das Insolvenzrecht soll zwar einen angemessenen Gläubigerschutz gewährleisten, aber nicht die zweite Chance mit einem Neustart verhindern. Entrepreneurship-Trainings und Gründerparks an Universitäten, erleichterte Arbeits- und Aufenthaltsbewilligung für gründungswillige Personen und Bürokratieerleichterungen wie z.B. One-Stop-Shops können Gründungen erleichtern.

(4) Steuerliche Diskriminierung des Risikokapitals abbauen. Je ehrgeiziger die Innovationen und je größer die Risiken sind, desto mehr braucht es Risikokapital. Um mehr Risikokapital zu mobilisieren, soll die Besteuerung einen vollen Verlustausgleich und Verlustvortrag auf Investorenseite garantieren und auf Unternehmensebene den Steuerabzug von kalkulatorischen Eigenkapitalzinsen zulassen. 
(5) Fiskalische F\&E-Förderung erhalten. Erstens schafft private F\&E neben eigenen Erlösen zusätzliche unentgeltliche Erträge bei anderen Nutzern. F\&E-Förderung soll diese nach dem Verursacherprinzip kompensieren. Zweitens ist es sinnvoll, den Nachteil des kleinen Binnenmarktes auszugleichen und ausländische Begünstigungen mit eigener F\&EFörderung wettzumachen. Drittens bauen F\&E-Steuerabzüge gezielt Finanzierungsprobleme dort ab, wo sie am häufigsten vorkommen, nämlich bei jungen innovativen Wachstumsfirmen. Daher sollte die Forschungsprämie generös ausgestaltet bleiben.

(6) Wagnisfinanzierung stärken. Wagniskapitalisten finanzieren hoch riskante Innovationen, wo Banken meist keinen Kredit mehr geben. Sie stärken zudem das Unternehmenswachstum mit strategischer Beratung und Überwachung. Angesichts des viel zu kleinen Kapitalmarkts sollte die aws wenigstens vorübergehend das Fund-in-Fund Geschäft stark ausbauen und sich mehr in privaten Beteiligungsgesellschaften engagieren, die ihrerseits die Portfolio-Investments auswählen und betreuen. Alle Maßnahmen zur Stärkung des Kapitalmarktes würden der Wagnisfinanzierung zusätzlichen Schub geben.

(7) Kapitalmarkt und Börse stärken. Eigenkapitalfinanzierung erfolgt über den Kapitalmarkt. Ein Bündel von Reformen ist notwendig, um den Finanzplatz auf zwei starke Beine zu stellen: leistungsfähige Banken und liquide Kapitalmärkte. Neben der Beseitigung von steuerlichen Hindernissen, einem starken Kapitalmarktrecht für mehr Rechtssicherheit, und einer Verbesserung des Finanzwissens über gute Anlageprinzipien müsste ein moderater Aufbau einer kapitalgedeckten Säule der Pensionsversicherung erfolgen. Ein wesentlich größeres Anlagevolumen kann nur von institutionellen Großanlegern kommen.

(8) Märkte offen halten. Wenn der Zugang zu den in- und ausländischen Absatzmärkten versperrt ist, machen Innovationen wenig Sinn. Ein leichterer Marktzugang erfordert ein starkes Wettbewerbsrecht mit einer klaren Insolvenzordnung, eine weniger kostspielige Regulierung, und eine schnelle und unbürokratische Abwicklung von Gründungen. Der Binnenmarkt ist für innovative Firmen viel zu klein, um stark zu wachsen. Deshalb müssen die Weiterentwicklung der EU-Mitgliedschaft sowie der Abbau von Handelshemmnissen mit anderen Handelszonen ein wichtiger Bestandteil einer Innovationsstrategie sein.

(9) Reallokation und Strukturwandel erleichtern. Innovation verursacht Strukturwandel. Die Faktorreallokation macht etwa die Hälfte des Produktivitätswachstums aus. Die Defizite des Kapitalmarkts behindern die Reallokation des Kapitals und sind zu beseitigen. Auf dem Arbeitsmarkt sind ein moderater Kündigungsschutz und eine ausgebaute aktive Arbeitsmarktpolitik mit dem Wandel gut vereinbar. Angefangen von der Stärkung der IKTKompetenzen in Schulen bis hin zur Umschulung und Weiterbildung für ältere Arbeitende (lebenslanges Lernen) kann die Bildungspolitik die Innovationsprozesse unterstützen.

(10) Innovationsstandort für multinationale Unternehmen sichern. Die multinationalen Unternehmen sind besonders F\&E-intensiv und spielen eine zentrale Rolle im Innovationssystem. Bei zunehmender Globalisierung wird die Standortattraktivität wichtiger. Die Präsenz von Spitzenuniversitäten wie auch die Verfügbarkeit von hoch qualitativem Humankapitel sind zentrale Standortvorteile für die F\&E-Einheiten multinationaler Unternehmen. Neben fiskalischen Maßnahmen ist daher eine Stärkung der universitären Ausbildung und der Grundlagenforschung vordringlich. 


\section{Einleitung}

Innovation treibt das Wachstum der Unternehmen und der Volkswirtschaft. Am Beginn stehen die Grundlagenforschung und die universitäre Ausbildung an der Grenze der Forschung. Diese erbringen Vorleistungen für die private Forschung und Entwicklung (F\&E) wie neue Anwendungen, Patente und hoch qualifiziertes Forschungspersonal. Mit Produktund Prozessinnovationen der privaten F\&E erringen die Unternehmen jenen Qualitätsvorsprung bzw. Kostenvorteil, welcher ihnen auf dem Markt eine höhere Wettbewerbsfähigkeit verschafft und private Investitionen erst rentabel macht.

Ökonomen beklagen ein Investitionsdefizit der österreichischen Wirtschaft und sehen die Ursache häufig in einem Verlust an Wettbewerbsfähigkeit und Standortattraktivität. Ist das Investitionsdefizit auf ein Innovationsdefizit zurückzuführen? Wie gut ist der F\&E-Standort Österreich positioniert? F\&E-Intensität und Investitionen werden oft isoliert betrachtet. Die Studie will beide Fragen verbinden und untersucht speziell die Standortattraktivität Österreichs für industrielle F\&E-Aktivitäten. Der Fokus auf den industriellen Sektor liegt nahe, weil die Industrie nach wie vor die F\&E-Aktivitäten in Österreich dominiert. Außerdem hat sich die Politik zum Ziel gesetzt, dass (i) Österreich bis 2020 zum Innovation Leader aufsteigen soll und (ii) die EU den Anteil der Industrie an der Wertschöpfung bis dahin auf 20 Prozent steigern will. In den letzten 20 bis 30 Jahren haben die europäischen Industriestaaten unterschiedliche Wege genommen: Während in manchen Ländern wie Großbritannien und Frankreich der Industrieanteil an der Bruttowertschöpfung deutlich abgenommen hat, ist er in anderen wie z.B. Österreich annähernd konstant geblieben. Eine Ausweitung der industriellen Produktion in anderen EU-Mitgliedstaaten verschärft jedoch den Wettbewerb und fordert auch den Industriestandort Österreich heraus.

Innovation steigert die Profitabilität und beschleunigt das Wachstum der Unternehmen. Für besonders innovative Firmen wird der Heimmarkt rasch zu klein, insbesondere wenn es sich um eine Marktnische handelt. Spezialisierte und innovative Firmen müssen daher den Weltmarkt erschließen, um ihr Wachstumspotenzial auszuschöpfen. Dort treffen sie auf eine weltweite Konkurrenz und können sich zumeist nur mittels eines überdurchschnittlichen Innovationsgrads behaupten. Im Querschnitt sind exportorientierte heimische Produzenten und multinationale Unternehmen daher auch überdurchschnittlich innovativ und produktiv.

Weil sie mit Exporten ausländische Märkte bearbeiten, sind innovative Unternehmen überaus mobil und können leicht den Standort verlegen, um direkt am Absatzort zu produzieren. Standortattraktivität ist für die produktivsten und innovativsten Unternehmen mit dem größten Wachstumspotenzial besonders wichtig. Für die Beschäftigung ist entscheidend, dass Unternehmen nicht nur die F\&E in Österreich durchführen, sondern der Standort auch für die industrielle Produktion attraktiv bleibt. Standortattraktivität ist nicht nur für die Unternehmen als Ganzes, sondern für einzelne Unternehmensfunktionen wie F\&E, Produktion, Vertrieb, Finanzierung und Konzernführung im Hauptsitz separat zu überprüfen. Die Wertschöpfungskette besteht aus vielen kleinen Schritten. Der Trend zur digitalen, webbasierten Wirtschaft macht es leichter, die Wertschöpfungskette weltweit zu verteilen, um Kosten zu sparen oder andere Standortvorteile zu nutzen. Große Teile der Wertschöpfung können ausgelagert und aus dem Ausland zugekauft werden, sodass im Inland nur „zusammengeschraubt und verpackt" wird. 
Die Digitalisierung der Wirtschaft und die zunehmende Automatisierung standardisierter Arbeitsschritte lösen einen kräftigen Strukturwandel aus und fordern sowohl Unternehmen als auch Bildungs- und Forschungspolitik heraus. So werden Unternehmen mit großem F\&EAufwand neue Geschäftsmodelle entwickeln und traditionelle Produktionsmodelle ersetzen. Die Digitalisierung, die Nutzung großer Datenmengen und der Einsatz von Robotern ermöglichen große Produktivitäts- und Einkommenszuwächse. Die Anforderungen an die Arbeit ändern sich stark. Neue und bessere Qualifikationen werden notwendig. Roboter können eine Reihe von Arbeitsschritten ersetzen. Viele berufliche Tätigkeiten werden sich stark verändern. Völlig neue Berufe entstehen, während manche traditionelle Berufe ganz verschwinden. Diese Entwicklung kann nicht verhindert werden. Am Ende werden jene Länder in der wirtschaftlichen Entwicklung am meisten vorankommen, welche den dadurch ausgelösten Strukturwandel aktiv unterstützen und nicht bremsen. Die Wirtschaftspolitik ist daher angehalten, die notwendigen Anpassungen frühzeitig zu antizipieren und die neuen Anforderungen an die Bildungs- und Forschungspolitik einschließlich der Bedeutung der Grundlagenforschung rasch zu klären.

Je mehr und je grundlegender die Innovationen ausfallen, desto stärker ist der Strukturwandel, der sowohl innerhalb etablierter Unternehmen wie auch zwischen den Unternehmen durch Marktzutritt und Austritt erfolgt. Große Konzerne müssen ihren Produktzyklus erneuern, Produktion und Beschäftigung umstellen und mit ständiger Innovation ihre Marktstellung behaupten und ausbauen. Durch Marktaustritt und Neugründungen entwickelt sich der Unternehmenssektor weiter. Kapital und Arbeit werden neu eingesetzt. Die Produktivität steigt, wenn Kapital und Arbeit gezielt in die ertragreicheren Aktivitäten fließen. Damit sich Innovationen durchsetzen, benötigen Banken und Kapitalmärkte eine hohe Fähigkeit, die Kapitalverwendung zu überwachen und Finanzierung von schrumpfenden auf wachsende Branchen und Unternehmen zu lenken.

Die Grundlagenforschung und tertiäre Ausbildung erbringen kritische Vorleistungen, stärken die Rentabilität und die Wirksamkeit der privaten F\&E und stoßen mehr industrielle Innovation an. Private F\&E treibt das Unternehmenswachstum, wobei innovative Unternehmen rasch durch Export in ausländische Märkte vorstoßen müssen, um wachsen zu können. Je größer die Unternehmen und je bedeutender die Auslandsmärkte für die weitere Entwicklung werden, desto eher gründen sie ausländische Niederlassungen und entwickeln sich zu großen Konzernen, die in vielen Ländern aktiv sind. Multinationale Unternehmen sind üblicherweise die produktivsten und forschungsintensivsten. Gerade wegen ihrer anhaltenden Innovationsfähigkeit sind sie so groß und produktiv geworden. Wegen ihrer Größe sind sie am ehesten selbst in der Grundlagenforschung aktiv, weil sie die ex ante unbekannten Ergebnisse der Grundlagenforschung potenziell in einer Vielzahl von Produktlinien anwenden können. Die großen Konzerne suchen daher häufig die Kooperation mit Universitäten und anderen Zentren der Grundlagenforschung und sind die wichtigsten Geldgeber für die industrielle Drittmittelfinanzierung der Universitäten. Diese Rückkoppelung schließt den Innovationskreislauf, wobei die dunkelblauen Pfeile in Abbildung 1 kausale Effekte andeuten.

Mit Prozessinnovationen erhöhen die Unternehmen ihre Produktivität und steigern ihre preisliche Wettbewerbsfähigkeit. Mit Produktinnovationen bauen sie ihre Marktanteile aus oder erringen die Marktführerschaft auf ganz neuen Märkten. Erst dadurch werden 
Investitionen und Beschäftigung rentabel. In allen Phasen der Innovationskette stellt sich dabei die Standortfrage, wie die grauen Pfeile in Abbildung 1 anzeigen. Universitäten konkurrieren weltweit um die StarforscherInnen und größten Talente unter den Studierenden. Darüber hinaus zeigt sich die Wettbewerbsfähigkeit der Grundlagenforschung auch in der Fähigkeit zur Anwerbung internationaler Forschungsgelder z.B. im Rahmen der EU-Forschungsprogramme. Die privaten F\&E-Einheiten der Unternehmen können in verschiedenen Ländern angesiedelt werden. Unternehmen können aus dem Inland durch Export die Weltmärkte beliefern oder abwandern, indem sie die Produktion ins Ausland nahe an den Absatzmärkten verlegen. Die Standortattraktivität bestimmt, wie viel an Investition oder Produktion im Inland oder im Ausland stattfindet.

Abbildung 1: Innovation, Unternehmensentwicklung und Standortattraktivität

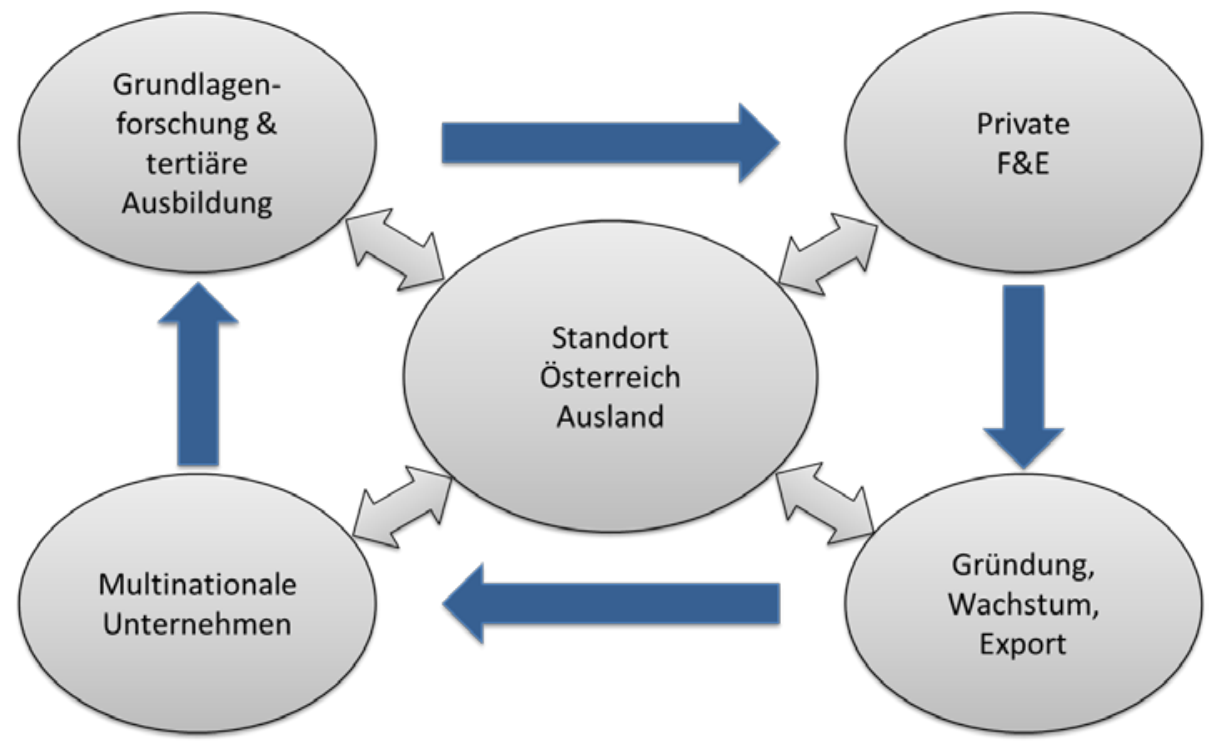

Quelle: eigene Darstellung

Abbildung 1 dient als organisierendes Prinzip für die vorliegende Studie. Kapitel 2 zeichnet ein statistisches Porträt vom Innovationsstandort Österreich. Wie stellt sich die Entwicklung im Vergleich zu anderen Innovationsländern dar? Kapitel 3 arbeitet die Bedeutung privater F\&E für die Unternehmensentwicklung heraus. Wie weit können höhere F\&E-Ausgaben mehr Investitionen in Ausrüstung und Anlagen anstoßen und das Unternehmenswachstum multiplizieren? Wie stark erhöhen F\&E-Ausgaben die Wahrscheinlichkeit, ausländische Märkte zu erschließen und die Exporttätigkeit aufzunehmen? Wie weit sind F\&E-Aktivitäten und industrielle Produktion gekoppelt oder können sie an verschiedenen Standorten angesiedelt werden? Kapitel 4 geht der Bedeutung der wirtschaftspolitischen Rahmenbedingungen für die industriellen F\&E-Aktivitäten in Österreich nach. Wieweit wirksam sind fiskalische F\&E-Anreize wie Steuerabsetzbeträge, Patentboxen und direkte Subventionen? Ist es möglich, mit fiskalischer Förderung gezielt die innovativsten und international mobilsten Unternehmen zu erreichen und damit das öffentliche Budget zu schonen? Wie kann Wagnisfinanzierung die Wirkung der F\&E-Ressourcen steigern und welche Maßnahmen wären notwendig, um die Wagnisfinanzierung in Österreich zu beleben? Kapitel 5 zieht Schlussfolgerungen und leitet Handlungsempfehlungen ab. 


\section{Innovationsland Österreich: Ein statistisches Porträt}

Österreich hat in den letzten beiden Jahrzehnten $\mathrm{zu}$ den führenden Innovationsländern aufgeschlossen. Die EU klassifiziert Dänemark, Deutschland, Finnland, die Niederlande und Schweden als Innovation Leader. Ziel ist es, in diese Gruppe vorzustoßen. Bei einigen Kennzahlen kann Österreich mit den führenden Ländern mithalten oder liegt sogar vor ihnen. Eine Reihe von Schwächen lässt jedoch den Innovations-Output trotz hoher F\&E-Quote relativ gering ausfallen. Das Kapitel identifiziert Stärken und Schwächen und stellt die Position im Innovationswettbewerb dar. Es beschreibt mit wichtigen Inputfaktoren die F\&E-Intensität, wendet sich dem Erfolg (Output-Indikatoren) zu und geht zuletzt auf die Finanzierung ein.

\subsection{F\&E-Aufwand}

Das Innovationspotenzial hängt von den F\&E-Investitionen ab, deren Erfolg den Forschungsoutput wie Erfindungen, Patente und wissenschaftliche Publikationen bestimmt. Typische Input-Faktoren sind F\&E-Ausgaben, das Forschungspersonal sowie der Aufwand für Grundlagenforschung. In Österreich scheint es allerdings Probleme mit der Produktivität des Innovationssystems zu geben. Obwohl das Land eine der höchsten F\&E-Quoten in der EU aufweist, fallen einige Ergebniskennzahlen gegenüber führenden Innovationsländern ab.

\subsubsection{Entwicklung der Forschungsquoten}

Die Forschungs- bzw. F\&E-Quote misst die betrieblichen F\&E-Ausgaben in Prozent des BIP. Die EU hat sich im Rahmen ihrer „Europa 2020“-Strategie zum Ziel gesetzt, im Jahr 2020 eine Forschungsquote von 3\% erreicht zu haben, ausgehend von 1,93\% in 2010.

Abbildung 2: F\&E-Quote Österreichs im Vergleich mit den führenden Industriestaaten, 1995-2015

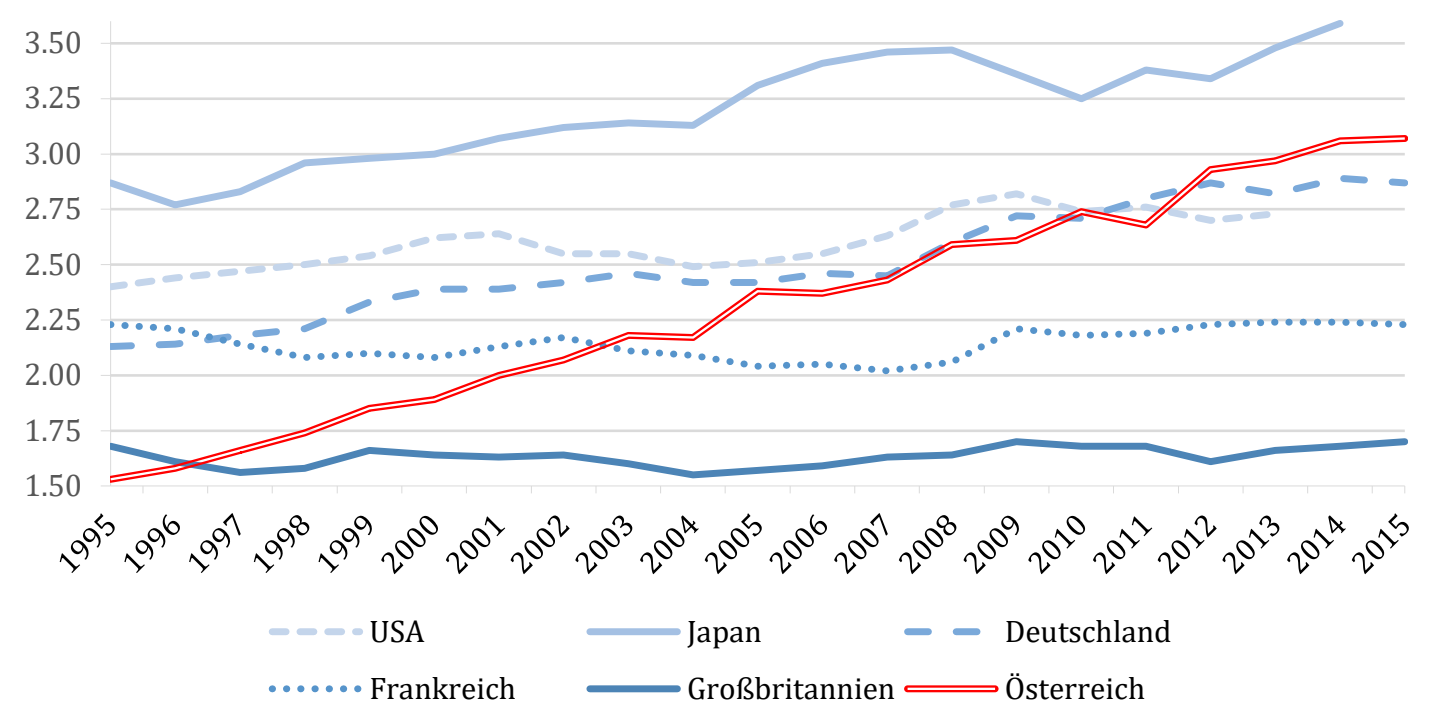

Anmerkung: USA 2014, 2015, Japan 2015: keine Daten

Quelle: Eurostat, eigene Darstellung 
Die Bundesregierung strebt im Rahmen der Europa-2020-Strategie für Österreich sogar eine Forschungsquote von 3,76\% im Jahr 2020 an (FTI-Strategie der Bundesregierung vom Jahr 2011), was nahezu einer Verdopplung innerhalb eines Jahrzehnts entspräche. Dieses Ziel erscheint sehr ambitioniert. Jedoch hat Österreich bereits in den letzten 20 Jahren bemerkenswert zu den führenden Industriestaaten aufgeholt. Nach Abbildung 2 konnte Österreich seit dem EU-Beitritt 1995 seine Forschungsquote stetig erhöhen und hat Deutschland, Frankreich, Großbritannien und die USA überholt. Lediglich Japan scheint vorerst außer Reichweite. Österreich befand sich hinsichtlich des BIP je Einwohner bereits auf recht hohem Niveau und verzeichnete zumindest bis 2008 ein recht hohes Wachstum. Umso bemerkenswerter ist die Steigerung der F\&E-Quote. In absoluten Zahlen hat sich das F\&E-Volumen von 1995 bis 2012 inflationsbereinigt mehr als verdoppelt.

Abbildung 3: F\&E-Intensität des Verarbeitenden Gewerbes, 2009-2013

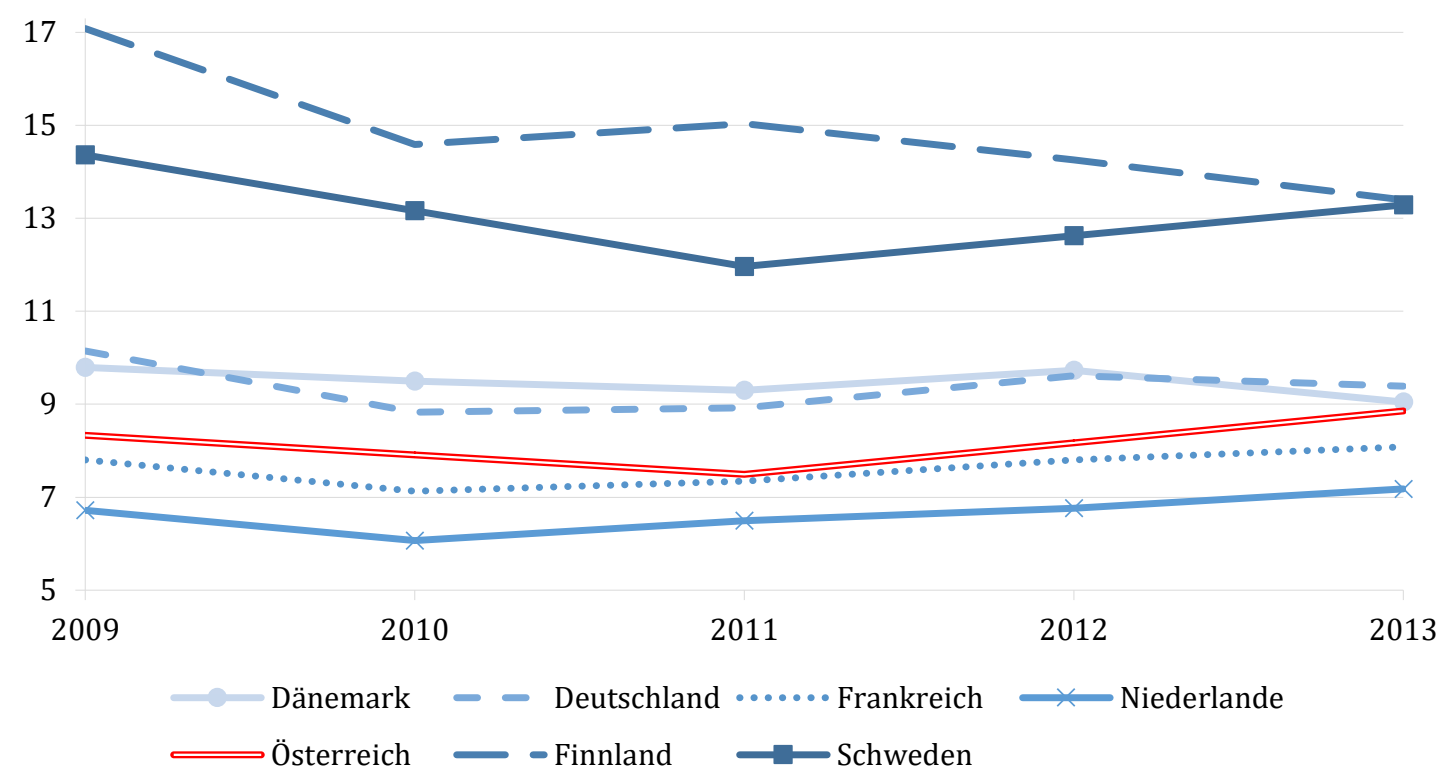

Quelle: Eurostat, eigene Berechnungen und Darstellung

In Österreich gaben dabei die Betriebe des Verarbeitenden Gewerbes im Jahr 2013 durchschnittlich 8,9\% der Bruttowertschöpfung für F\&E-Investitionen aus.1 Die F\&EIntensität lag damit nach Abbildung 3 in etwa gleichauf mit Dänemark und deutlich vor den Niederlanden und Frankreich. In Schweden und Finnland sind dagegen die Betriebe deutlich forschungsintensiver. Während Österreich seit 2012 bei der F\&E-Quote vor Deutschland liegt, ist die F\&E-Intensität des Verarbeitenden Gewerbes nach wie vor geringer. Ähnlich liegt der

1 Das Verarbeitende Gewerbe (NACE-Kode, Rev. 2: C) stellt den Großteil der Industrie und erzielte in Österreich 2013 18,5\% der gesamten Bruttowertschöpfung (Statistik Austria). Zur Industrie im weiteren Sinn zählen auch der Bergbau (NACE-Kode B, Anteil der Bruttowertschöpfung 0,5\%), Energieversorgung (D, 1,8\%), Wasser- und Abfallentsorgung (E, 1,1\%) und das Baugewerbe (F, 6,4\%). Die Studie fokussiert auf das Verarbeitende Gewerbe, da es den größten Teil des industriellen Sektors einnimmt und der Vorstellung hochproduktiver industrieller Produktion, getrieben von Innovation und Investitionen, am nächsten kommt. Die Studie verweist bei empirischen Angaben stets auf die Datengrundlage („Verarbeitendes Gewerbe“, oder „gesamter industrieller Sektor“, „Industrie ohne Baugewerbe“ etc.), gebraucht jedoch im verbalen Teil die Begriffe synonym. 
Fall Schweden. Die F\&E-Quote 2013 übertraf jene von Österreich um ca. 12\%, die F\&EIntensität des Verarbeitenden Gewerbes lag dagegen um etwa 50\% höher. Diese Unterschiede haben mit dem kleineren Anteil von Firmen mit Spitzentechnologie zu tun.

\subsection{2 Österreichs Position in der Grundlagenforschung}

Die meisten Länder entwickeln neue Technologien nicht selber, sondern übernehmen sie weitgehend von den Innovationsführern (Acemoglu, 2009). Je mehr jedoch ein Land zu den technologisch führenden Staaten aufholt, umso mehr muss es die Technologiegrenze selbst hinausschieben. Es genügt immer weniger, bestehende Technologien partiell zu verbessern. Die Grundlagenforschung wird daher wichtiger. Sie schafft die Grundlagen für neue Produkte und Anwendungen, die später revolutionären Charakter haben. Der Erfolg der Grundlagenforschung ist jedoch höchst ungewiss. Die Erkenntnisse sind ohne Rivalität von allen nutzbar. Es ist nur schwer möglich, die Erträge auf die investierenden Unternehmen zu beschränken und jene auszuschließen, die nicht dafür zahlen wollen. In Folge wird die Privatwirtschaft zu wenig investieren, so dass der Staat einspringen muss, um dieses öffentliche Gut zu finanzieren. Daher erfolgt die Grundlagenforschung vorwiegend an den Universitäten, während die Unternehmen nur wenig investieren (Abbildung 4).

Abbildung 4: Ausgaben für F\&E nach Durchführungssektoren und Forschungsarten, Mio. Euro, 2013

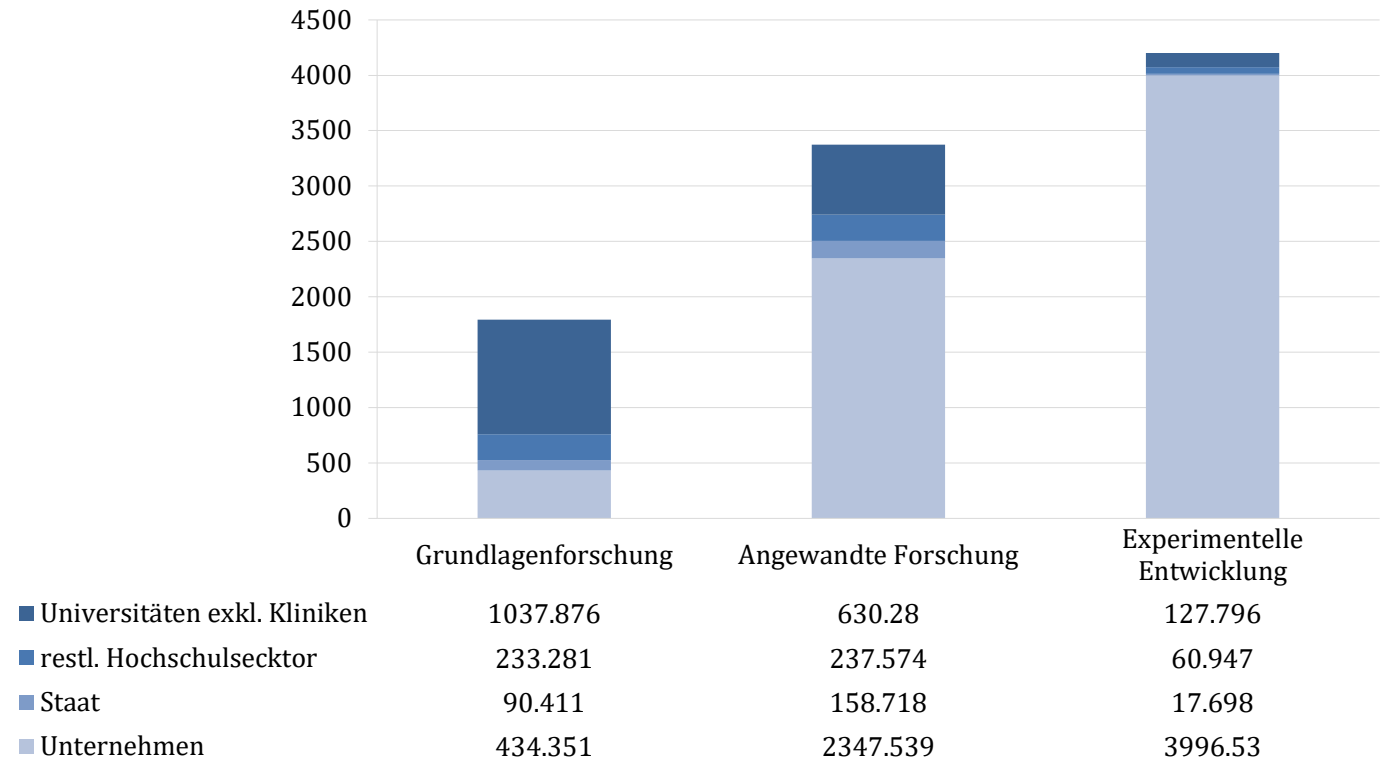

Anmerkung: wegen seiner geringen Bedeutung ohne privaten gemeinnützigen Sektor Quelle: Statistik Austria, eigene Berechnungen

Der Anteil Grundlagenforschung an den gesamten F\&E-Ausgaben ist von ca. 17\% Mitte der 2000er auf 19\% während der 2010er-Jahre gestiegen. Zwar liegt Österreich bei den F\&EAusgaben im internationalen Spitzenfeld, beim Anteil der Grundlagenforschung am BIP hinkt es jedoch deutlich den führenden Ländern wie Japan oder der Schweiz hinterher. Auch der Anteil der Grundlagenforschung an den gesamten F\&E-Ausgaben ist deutlich niedriger als in Ländern mit vergleichbaren F\&E-Quoten wie z.B. Frankreich (2013: 24,3\%) oder den 
Niederlanden (2013: 28,7\%, nach Eurostat). Ein Grund für diese Schwäche liegt wohl darin, dass in Österreich relativ wenige große, multinationale Konzerne vertreten sind, welche am ehesten selbst in Grundlagenforschung investieren können. Umso mehr ist der Staat gefordert, eine Ausweitung der Grundlagenforschung zu finanzieren. Die Bereitstellung öffentlicher Güter gehört auch in einer Marktwirtschaft zu den klassischen Staatsaufgaben.

\subsubsection{F\&E nach Betriebsgrößen und Sektoren}

Mit 62,0\% entfiel in 2013 der überwiegende Anteil der von Unternehmen durchgeführten F\&E-Investitionen auf das Verarbeitende Gewerbe. Allerdings hat sich dieser Anteil seit 2004 um 9,1 Prozentpunkte reduziert, während der F\&E-Anteil des Dienstleistungssektors von 27,4\% auf 37,0\% gestiegen ist.2 Die F\&E-Intensität im Sachgüterbereich fällt mit 7,9\% deutlich höher aus als im Dienstleistungsbereich mit 1,2\%. Sowohl das Verarbeitende Gewerbe als auch der Dienstleistungssektor konnten die F\&E-Intensität seit 2004 gegenüber den damaligen Werten von 6,1\% bzw. 0,7\% deutlich steigern (Statistik Austria). Etwa 40,5\% der F\&E-Ausgaben entfallen auf Betriebe mit mindestens 1.000 Beschäftigten. Die Tochtergesellschaften ausländischer Konzerne tätigen einen hohen Anteil der privaten F\&EInvestitionen und haben stark zum Aufholprozess Österreichs beigetragen (Dachs, 2016).

Abbildung 5: Anteile des Verarbeitenden Gewerbes an BWS und F\&E-Ausgaben, EU15-Staaten, 2014

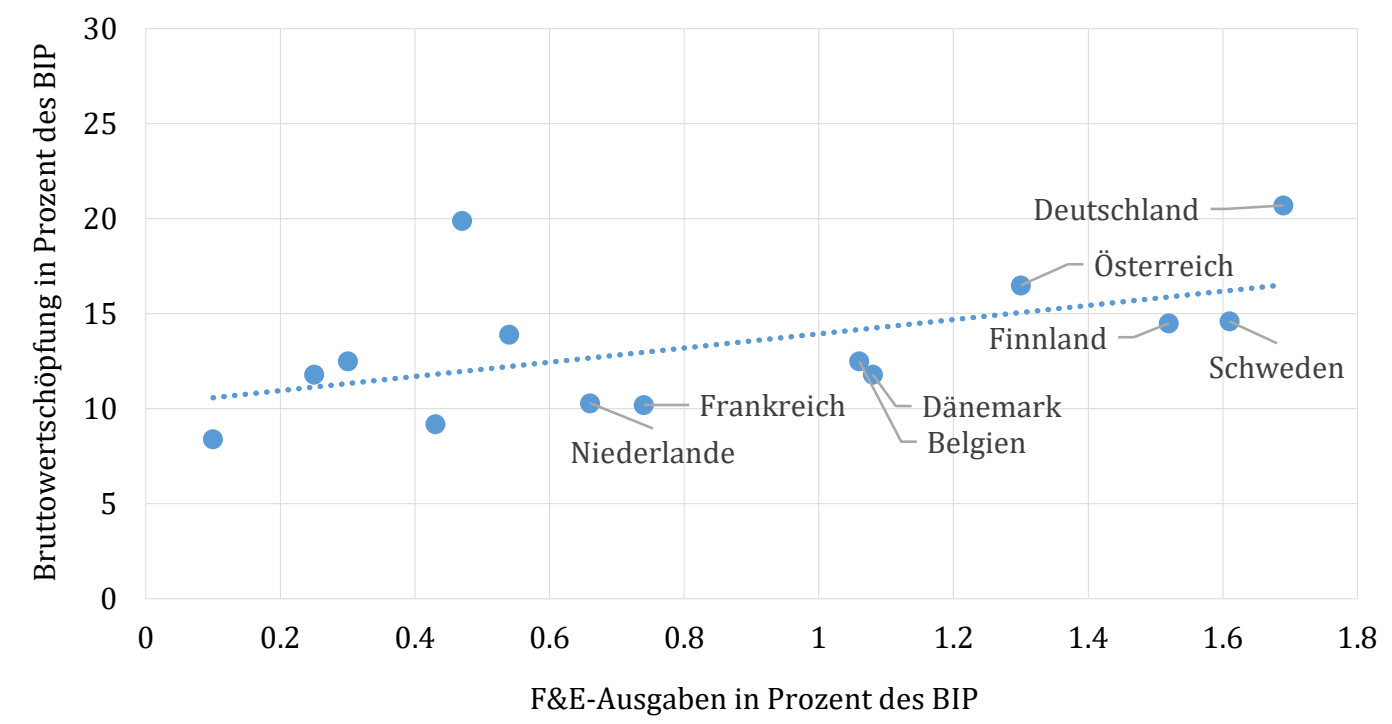

Anmerkung: Belgien, Irland, Frankreich, Griechenland, Österreich, Schweden: Daten für 2013; Luxemburg: keine Daten

Quelle: Eurostat, eigene Darstellung

Österreichs Stärke liegt im mittleren Technologiesegment (BMWF u.a., 2013). Auf dieses Segment entfällt relativ zu führenden Industriestaaten ein hoher Anteil der industriellen Wertschöpfung wie auch der F\&E-Ausgaben. Viele Unternehmen sind in Nischenbereichen erfolgreich. Die hohe F\&E-Intensität mag sich zumindest teilweise durch den vergleichsweise hohen Industrieanteil in Österreich erklären. Ein Indiz dafür ist, dass in Ländern wie

2 Der Dienstleistungssektor umfasst alle Branchen außer dem Agrar- und Industriesektor. Die auf 100\% fehlenden 1,0\% entfallen auf den Agrarsektor (2013: 0,05\%) sowie die nicht zum Verarbeitenden Gewerbe zählenden industriellen Zweige. 
Großbritannien, wo der Anteil der Industrie an der Bruttowertschöpfung deutlich fällt, tendenziell auch der Anteil des Unternehmenssektors an den F\&E-Ausgaben abnimmt. Hinzu kommt, dass alle von der EU als Innovation Leader klassifizierten Länder einen großen Industrieanteil aufweisen, wie Abbildung 5 für die EU15-Staaten zeigt.3 Je größer der Anteil des verarbeitenden Gewerbes an der gesamten Wirtschaftsleistung, umso höher sind tendenziell auch die F\&E-Ausgaben. Der Korrelationskoeffizient der Daten in Abbildung 5 beträgt 0,54. Österreich befindet sich damit in guter Gesellschaft der Innovation Leader und liegt dabei leicht über der Trendlinie. Im Verhältnis zu seinem F\&E-Anteil ist der BIP-Anteil der Industrie relativ niedrig. Diese Befunde belegen die hohe Bedeutung der Industrie für nationale Innovationssysteme (BMWFW, 2014b). Allerdings sind die USA als technologisch führende Volkswirtschaft durch eine weitgehende De-Industrialisierung gekennzeichnet. Es sind also auch alternative Entwicklungspfade möglich.

\subsubsection{Humankapital und Forschungspersonal}

Obwohl die Akademikerquote (Anteil der Bevölkerung mit tertiärem Abschluss) deutlich unter dem Durchschnitt der EU liegt, konnte Österreich seine F\&E-Quote in den letzten zwei Jahrzehnten stark steigern. Die Industrie benötigt im mittleren Technologiesegment offenbar keine hohe Akademikerquote, um international wettbewerbsfähig zu sein (BMWF u.a., 2013). Eine Variation der Akademikerquote hat in Österreich auch kaum einen Einfluss auf das regionale Beschäftigungswachstum (Sardadvar und Reiner, 2016).

\section{Abbildung 6: Akademikerquoten und F\&E-Personal in den EU-Mitgliedstaaten, 2014}

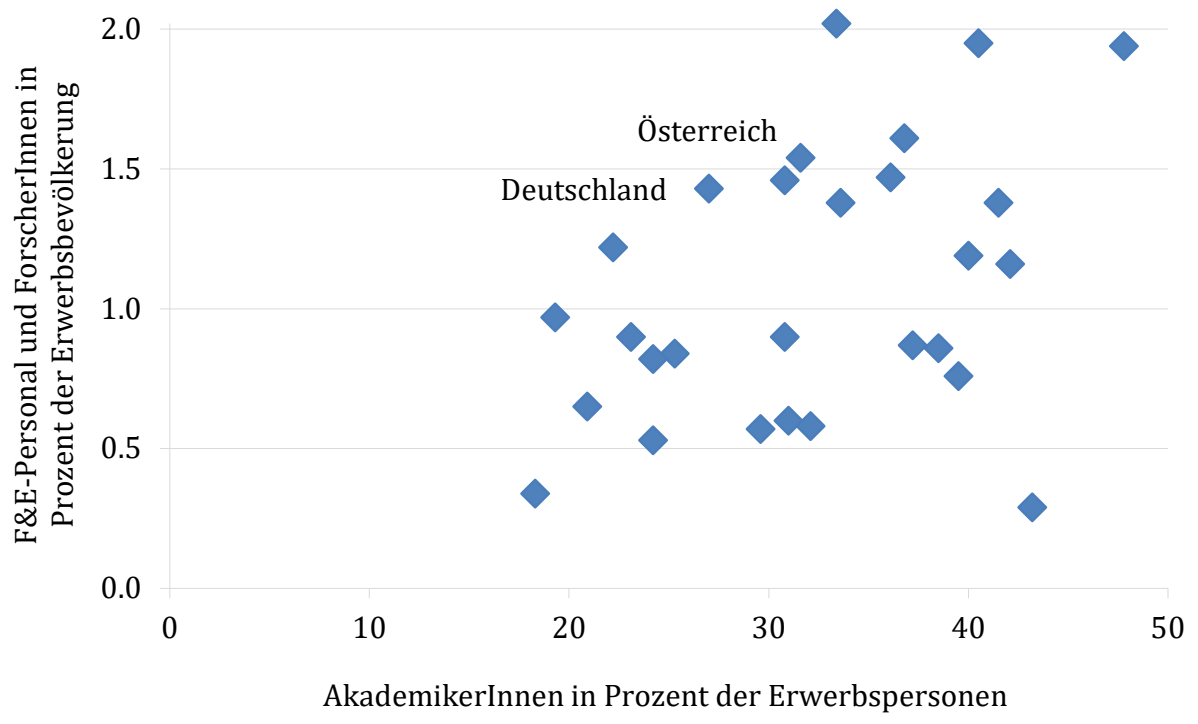

Quelle: Eurostat, eigene Darstellung

Die wissenschaftliche Literatur sieht dagegen in der Akademikerquote einen wichtigen Faktor für die Innovationsfähigkeit eines Landes. Die offensichtliche Schwäche dieses Indikators ist, dass nur ein Bruchteil der AkademikerInnen tatsächlich in der Forschung tätig

3 Mit „EU15“ sind jene 15 Mitgliedstaaten gemeint, welche der EU vom 1. Jänner 1995 bis zum 30. April 2004 angehört haben. 
ist. Österreich besetzt in der EU bei der Akademikerquote nur Rang 15, in der Kategorie „F\&EPersonal und ForscherInnen in Prozent der Erwerbsbevölkerung" jedoch Rang 5. Ähnliches gilt auch für Deutschland. Abbildung 6 zeigt zwar für 28 EU-Mitgliedstaaten einen positiven Zusammenhang, der Korrelationskoeffizient ist jedoch mit 0,4 nicht besonders hoch.

Deutschland und Österreich haben gemeinsam, dass sie über ein sehr gutes Ausbildungssystem im sekundären Bereich verfügen, was in anderen Ländern oft über die tertiäre Ausbildung kompensiert wird (Culpepper, 2007). Zwar sind AkademikerInnen für den Einsatz in F\&E zweifellos unverzichtbar. Für Österreich stellt sich allerdings eher die Frage, welche AkademikerInnen ausgebildet und angeworben werden sollen.

Um die Grundlagenforschung weiter zu stärken, hat die Regierung eine Reihe von Maßnahmen mit dem Ziel der Förderung und Steigerung der „Exzellenz“ angekündigt. Die FTI-Strategie nennt dabei den Ausbau der Drittmittelfinanzierung der Hochschulforschung sowie die Einrichtung von Exzellenzclustern bis 2020 als Ziele. Vor allem sollen bestehende exzellente Forschungsgruppen gestärkt und ausgebaut werden (BMWFW und BMVIT, 2016). Eine fast schon chronische Schwäche Österreichs betrifft allerdings die finanzielle Ausstattung der Universitäten einschließlich der Zahl der ProfessorInnen. Tabelle 1 vergleicht Österreich und die Schweiz für ausgewählte Universitäten und insgesamt. Österreich wird dabei von der Schweiz in jeder Hinsicht deutlich übertroffen.

Tabelle 1: Vergleich Universitäten in Österreich und der Schweiz, 2013

\begin{tabular}{lllllll}
\hline & $\begin{array}{l}\text { TU } \\
\text { Wien }\end{array}$ & $\begin{array}{l}\text { ETH } \\
\text { Zürich }\end{array}$ & $\begin{array}{l}\text { Universität } \\
\text { Wien }\end{array}$ & $\begin{array}{l}\text { Universität } \\
\text { Zürich }\end{array}$ & $\begin{array}{l}\text { Österreich } \\
\text { gesamt }\end{array}$ & $\begin{array}{l}\text { Schweiz } \\
\text { gesamt }\end{array}$ \\
\hline Studierende & 27.111 & 17.309 & 91.362 & 26.351 & 299.355 & 138.624 \\
Budget (Mrd. $€$ ) & 0,3 & 1,1 & 0,5 & 0,5 & 3,6 & 5,4 \\
THE-Ranking & $\leq 250$ & 14 & 170 & 121 & - & - \\
Anzahl ProfessorInnen & 137 & 482 & 423 & 533 & 2.377 & 3.555 \\
Studierende/ProfessorIn & 198 & 36 & 216 & 49 & 126 & 39 \\
\hline
\end{tabular}

Bemerkung: Angaben für beide Länder ohne Fachhochschulen

Quellen: RFTE (2014), BMWFW (2014a), teilweise eigene Berechnungen

In Österreich mehren sich Anzeichen für eine Abwanderung von AkademikerInnen und Forschungspersonal. Ein Bericht der Statistik Austria (Wisbauer und Fuchs, 2014) deutet darauf hin, dass das Bildungsniveau der auswandernden Personen auffallend hoch ist. So weisen im Zeitraum 2010 bis 2012 Personen mit Hochschulabschluss die höchsten Wegzugsraten auf, gefolgt von Personen mit AHS-Matura. Nach Fachrichtung bzw. Ausbildungsfeld liegen die Naturwissenschaften an erster Stelle, nach dem Alter sind es die 25- bis 35-Jährigen. Umgekehrt wird der niedrige Anteil an hoch und höchst qualifizierten ZuwanderInnen beklagt. Österreich ist "das OECD-Land mit dem geringsten Anteil (11,3 Prozent) von AkademikerInnen unter den im Ausland geborenen Personen" (BMWF u.a., 2009).

Mögliche Gründe für die Schwäche, Forschungspersonal anzuwerben und zu halten, sind die schlechten Karriereoptionen an den Universitäten sowie vergleichsweise niedrige Gehälter in der Industrie. Nach Miguélez und Fink (2013) weist Österreich bei den 
Wanderungsströmen von ErfinderInnen im Zeitraum 2001 bis 2010 klar eine negative Nettoposition auf. Deutlich mehr als ErfinderInnen klassifizierte Personen haben das Land verlassen als zugezogen sind.

\subsection{F\&E-Erfolg}

Die Unternehmen gehen mit ihren F\&E-Investitionen ein hohes Risiko ein und müssen oft lange auf den Erfolg warten. Erfolgreiche Innovationen lassen die Unternehmen expandieren, Misserfolg führt zum Scheitern. Der Wettbewerb führt daher zu einem Ausleseprozess, der die Produktivität in der Gesamtwirtschaft steigert und das gesamtwirtschaftliche Wachstum unterstützt. Dabei nehmen Innovationen unterschiedliche Formen an. Produktinnovationen erschließen mit neuen Produkten neue Märkte oder bauen mit Qualitätssteigerungen die Marktstellung aus. Prozessinnovationen bzw. organisatorische Innovationen verbessern das Zusammenspiel in den Unternehmen und steigern die Produktivität der eingesetzten Ressourcen im Produktionsprozess. In der Tradition von Schumpeter erklärt die moderne Theorie innovatives Wachstum mit vier Ideen (vgl. Aghion und Howitt, 1992, und Aghion, 2016): (i) Produktivitätswachstum entsteht aus dem Profitstreben der Unternehmen; (ii) Innovationen führen zu schöpferischer Zerstörung („creative destruction“), indem sie mit neuen Produkten und Prozessen existierende Praktiken obsolet machen. Arbeit und Kapital müssen umgelenkt und neu eingesetzt werden; (iii) an der Technologiegrenze müssen Innovationen ganz neues Wissen und neue Technologien schaffen, im Aufholprozess finden Verbesserungen und Modifikationen vorhandener Technologien statt; (iv) der technologische Fortschritt ist zudem von bahnbrechenden Erfindungen (Schumpeterianische Wellen) geprägt, die zu einem neuen technologischen Paradigma, wie z.B. Digitalisierung und Industrie 4.0, führen.

\subsection{1 Österreich in internationalen Rankings}

Innovation hat viele Auswirkungen auf den Unternehmenserfolg mit neuen Produkten und Leistungen, auf Einkommen, Verteilung und Beschäftigung nach Qualifikationen, auf die internationale Spezialisierung der Wirtschaft und vieles mehr. Ein eher heuristischer, aber populärer Ansatz erfasst die Innovationsfähigkeit eines Landes mit einer Vielzahl von Kennzahlen und verdichtet diese in einem Ranking $\mathrm{zu}$ einem Gesamtbild. Der wissenschaftliche Wert ist eher zwiespältig, da die Gewichtung der verschiedenen Indikatoren teilweise willkürlich und die Positionierungen der Länder nicht robust sind. Andererseits können ähnliche Positionen in verschiedenen Rankings das Bild verfestigen, und es wird möglich, gewisse Trends im Zeitverlauf zu verfolgen. Details zu den Teilergebnissen können Aufschluss über spezifische Stärken und Schwächen geben.

Die EU gibt mit dem European Innovation Scoreboard (EIS, früher: Innovation Union Scoreboard) seit 2001 jährlich einen Ranking-Bericht heraus, der den politischen Einfluss solcher Reihungen veranschaulicht. So hat sich z.B. die Republik Österreich (2011) zum Ziel gesetzt, in diesem Bericht zu den Innovation Leader gezählt zu werden. Der aktuelle EISBericht führt Österreich in der zweiten von vier Klassen als sogenannten Strong Innovator (frühere Bezeichnung: „Innovation Follower") und damit noch vor den niedrigeren Kategorien Moderate Innovator und Modest Innovator. Damit zählt Österreich nach dem EIS zu jenen Mitgliedstaaten, deren Innovationleistung über oder nahe am EU-Durchschnitt liegt. 
Die Schweiz als Nicht-EU-Mitgliedstaat erfährt im EIS-Bericht Anerkennung als innovativstes Land Europas. Österreich liegt als Strong Innovator gleichauf mit Belgien, Frankreich, Großbritannien, Irland, Luxemburg und Slowenien. Zu den Innovation Leader aufgestiegen sind die Niederlande, die nun mit Dänemark, Deutschland, Finnland und Schweden die Bezugsgruppe für Österreich bilden. Vergleicht man im EIS 2016 Österreich mit dem Aufsteiger Niederlande, so fällt zuerst die chronische Schwäche bei der tertiären Bildung einschließlich Doktoratsstudierenden auf. Dafür liegt Österreich bei der sekundären Bildung vorne. Besonders schlecht ist das Ergebnis bei den Nicht-EU-Doktoratsstudierenden als Maßzahl für offene, exzellente Forschungssysteme. Diese Indikatoren zählen allerdings eher zu den Input-Faktoren. Recht gut hält sich Österreich bei den Output-Faktoren wissenschaftliche Publikationen und Zitaten, wobei die Niederlande hier zur Spitze gehören und noch besser abschneiden. Deutliche Schwächen zeigen die Niederlande hingegen bei den F\&E-Investitionen des Unternehmenssektors.

Klare Unterschiede zwischen den Niederlanden und Österreich treten bei den OutputFaktoren zutage. Obwohl Österreich bei den angemeldeten Patenten recht gut abschneidet, erzielen die Niederlande hier Spitzenwerte, insbesondere bei den Patenten zu Gesundheit und Klimawandel. Andererseits liegt Österreich bei eingetragenen Markennamen und Designs sogar vor der Gruppe der Innovation Leader. Besser als die Niederlande schneidet Österreich allgemein bei firmenbezogenen Kennzahlen ab, und im Speziellen bei den Kategorien „Anteil der KMU mit Marketing- oder organisatorischen Innovationen“ sowie bei der „Beschäftigung in schnell wachsenden Firmen innovativer Branchen“.

Bei den „wirtschaftlichen Auswirkungen“ schneidet Österreich im Allgemeinen wesentlich schwächer ab, nicht nur im Vergleich zu den Niederlanden, sondern auch zum EUDurchschnitt. Allerdings erzielt Österreich in zwei Subkategorien, nämlich „Exporte von Mittel- und Hochtechnologiebranchen“ sowie „Anteile neuer Produkte im Firmenumsatz" zwar im EU-Vergleich keine Spitzenwerte, liegt aber deutlich vor den Niederlanden. Schwächer als die Niederlande, aber über dem EU-Schnitt ist die Beschäftigung in wissensintensiven Branchen. Weit unter dem EU-Schnitt liegt Österreich hingegen bei Exporten wissensintensiver Dienstleistungen (die Niederlande liegen hier knapp über dem EU-Schnitt) sowie bei Lizenz- und Patent-Erträgen aus dem Ausland. In der letzten Kategorie nehmen die Niederlande eine Spitzenposition ein, während Österreich nicht nur abgeschlagen zurückliegt, sondern obendrein in den letzten acht Jahren ein negatives Wachstum aufweist.

Bei aller Vorsicht gegenüber Reihungen kristallisieren sich bei diesem Vergleich Stärken und Schwächen des Innovationslandes Österreich heraus. Österreich liegt nicht nur im EIS 2016 auf Platz zehn, sondern findet sich auch in anderen Reihungen, bereinigt um Nicht-EUMitglieder, in seinen Innovationsleistungen auf den Rängen sechs bis neun wieder (Global Competitiveness Report 2015/16, Subindex „innovation and sophistication factors“: Rang 7; Global Innovation Index 2015: Rang 9; Innovationsindikator der Deutschen Telekom Stiftung 2015: Rang 6). Somit fällt der Befund, dass sich Österreich als Innovationsland innerhalb der EU im vorderen Mittelfeld befindet, insgesamt recht robust aus. Eine Schwäche liegt jedoch darin, die hohen F\&E-Ausgaben in ebenso starken wirtschaftlichen Erfolg umzusetzen. 


\subsubsection{Patentierungen im internationalen Vergleich}

Patente bilden ein frühes Stadium der Wissensproduktion und der experimentellen Forschung $a b$, während andere Innovationen erst mit der kommerziellen Verwertung sichtbar werden. Patente gelten als Frühindikatoren von technologischen Entwicklungen (ISI, 2002). Allerdings können Patente nicht alle Forschungsleistungen erfassen, weil nicht alle Unternehmen ihre Erfindungen patentieren, und weil nach dem Europäischen Patentübereinkommen nicht jede Neuerung patentierfähig ist. Dazu zählen auch weite Teile der universitären Forschung wie z.B. allgemeine wissenschaftliche Theorien und mathematische Methoden. Auch ästhetische Gestaltungen wie Designs, die in vielen Branchen wesentliche Innovationen darstellen, sind nicht patentierbar. Für eine Patentierung nicht zugelassen sind z.B. auch Software und DNS-Kodes, die geradezu repräsentativ für den aktuellen technologischen Fortschritt sind.

Diese Einschränkungen können die Messung der Innovationsleistungen zuungunsten jener Länder verzerren, die überdurchschnittlich viele nicht patentierbare Forschungsoutputs erbringen. Zudem können die Kosten von Patentanmeldungen sowie der tatsächliche wirtschaftliche Wert von Patenten sich erheblich nach Branchen und Ländern unterscheiden und die Patenthäufigkeit beeinflussen. Die Patentierung ist eine strategische Entscheidung; oftmals werden Erfindungen bewusst nicht patentiert, weil mit einer Patentierung auch Informationen preisgegeben werden müssen, die lieber geheim gehalten werden. Aus diesen Gründen werden auch Prozessinnovationen tendenziell seltener patentiert. Die Neigung zur Patentierung unterscheidet sich stark zwischen Branchen, weshalb die Branchenstruktur eines Landes auch Auswirkungen auf die Zahl der Patente hat.

Tabelle 2 vergleicht für das Jahr 2014 Österreichs aufgewendete Arbeitsstunden, die gesamte Bruttowertschöpfung, die gesamten F\&E-Investitionen sowie sämtliche Patentanmeldungen mit der gesamten EU, den Innovation Leader, Frankreich und Großbritannien als weitere führende Industriestaaten sowie mit der Schweiz. Der österreichische Anteil an den F\&EAusgaben der EU liegt um 47\% (=(3,46/2,36-1)*100) höher als der Anteil an der BWS, was bedeutet, dass Österreich relativ zur Wirtschaftsleistung überproportional zur F\&E der EU beiträgt. Im Vergleich dazu liegt der Anteil der Innovation Leader an den F\&E-Investitionen nur um 37\% höher als ihr Anteil an der BWS. Der summierte Anteil von Frankreich und Großbritannien an den F\&E-Ausgaben der EU ist sogar niedriger als ihr Anteil an der BWS, obwohl diese Länder bestrebt waren, besonders forschungsintensive Industriezweige teilweise zulasten traditioneller Branchen auszubauen. Österreich liegt bei allen Kennzahlen erwartungsgemäß hinter der Schweiz. 
Tabelle 2: Produktion, F\&E, Patente im Vergleich, 2014

\begin{tabular}{lllll}
\hline & $\begin{array}{l}\text { Arbeitsinput } \\
\text { (Mio. Stunden) }\end{array}$ & $\begin{array}{l}\text { BWS } \\
\text { (Mrd. €) }\end{array}$ & $\begin{array}{l}\text { F\&E } \\
\text { (Mrd. €) }\end{array}$ & $\begin{array}{l}\text { Patente } \\
\text { (Anzahl) }\end{array}$ \\
\hline EU & 369.606 & 12.487 & 283,89 & 56.561 \\
Innovation Leader & 86.551 & 4.004 & 124,79 & 30.724 \\
Österreich & 6.941 & 294 & 9,83 & 1.958 \\
\hline Innovation Leader/EU & $23,42 \%$ & $32,07 \%$ & $43,96 \%$ & $54,32 \%$ \\
Österreich/EU & $1,88 \%$ & $2,36 \%$ & $3,46 \%$ & $3,46 \%$ \\
Österreich/Innovation Leader & $8,02 \%$ & $7,35 \%$ & $7,88 \%$ & $6,37 \%$ \\
\hline Großbritannien & 51.514 & 2.015 & 38,32 & 5.349 \\
Frankreich & 40.074 & 1.918 & 48,11 & 9.117 \\
Schweiz & 7.712 & 512 & 15,36 & 3.238 \\
\hline (GB + F)/EU & $24,78 \%$ & $31,49 \%$ & $30,45 \%$ & $25,57 \%$ \\
Österreich/Schweiz & $90,00 \%$ & $57,49 \%$ & $64,03 \%$ & $60,47 \%$ \\
\hline
\end{tabular}

Anmerkung: Innovation Leader sind Dänemark, Deutschland, Finnland, Niederlande und Schweden. Quelle: Eurostat, eigene Berechnungen

Besonders große Unterschiede treten bei den Patentanmeldungen hervor. Mehr als die Hälfte aller Patente, die beim Europäischen Patentamt 2014 angemeldet wurden, werden einem der fünf Innovation Leader zugeordnet, womit der Anteil um fast ein Viertel höher ist als der Anteil dieser Länder an den F\&E-Investitionen. Im Unterschied dazu sind die Anteile Österreichs bei Patenten und F\&E-Investitionen mit jeweils 3,46\% nahezu identisch. Im Gegensatz zu den Innovation Leader ist also die Ausbeute an Patenten im Verhältnis zum F\&EAufwand nicht hoch. Wären Patente ein perfekter Indikator für den F\&E-Erfolg, dann müsste man feststellen, dass Österreichs F\&E-Investitionen als Input wesentlich weniger Ertrag abwerfen als die F\&E-Investitionen in der Gruppe der Innovation Leader.

\subsubsection{Prozessinnovationen und Produktivität nach Branchen}

Die Arbeitsproduktivität ist einer der wichtigsten, nach Ansicht von Paul Krugman (1994) sogar der einzig relevante, Indikatoren für die Wettbewerbsfähigkeit. Sie misst den Output pro Einheit Arbeitsinput und folgt direkt aus den Prozessinnovationen. Aus Tabelle 2 ist bereits ein Maß der Produktivität ablesbar, nämlich BWS/Arbeitsstunden. Nachdem der Anteil Österreichs an der Bruttowertschöpfung (BWS) der EU mit 2,36\% den Anteil an den Arbeitsstunden von 1,88\% übersteigt, liegt die Arbeitsproduktivität über dem Schnitt der EU. Der Vorsprung beträgt 26\% (d.h. (2,36/1,88-1)*100). Allerdings ist der Vorsprung der Innovation Leader in der Arbeitsproduktivität mit 37\% deutlich größer, obwohl Österreich ein größeres BIP je Einwohner aufweisen kann als die meisten Innovation Leader. Der technologische Fortschritt und insbesondere die Prozessinnovationen sind zwar wichtige, jedoch nicht die einzigen Determinanten. Die Arbeitsproduktivität hängt auch von anderen Faktoren wie Infrastruktur, Kapitaleinsatz und Konjunktur ab. 
Abbildung 7 vergleicht die Produktivitätsentwicklung von 1998 bis 2015 mit jener in anderen Ländern und Regionen. Österreichs Produktivität ist schneller gestiegen als jene Frankreichs, Großbritanniens und der Schweiz. Die EU und Österreich liegen in 2015 praktisch gleichauf. Zusammen mit Tabelle 2 kann man daraus schließen, dass Österreich seinen absoluten Vorsprung gegenüber dem Schnitt der EU28 seit 1998 auf weitgehend gleichem Niveau hält. Das kumulierte Wachstum der Arbeitsproduktivität seit 1998 wird nur von Schweden nennenswert übertroffen, aber nicht von den anderen Innovationsführern. In Finnland und Schweden sind die F\&E-Quoten seit Mitte der 2000er zurückgegangen. Seitdem verzeichnet Schweden nur mehr ein geringes und Finnland sogar ein negatives Produktivitätswachstum. Auch in Dänemark, Deutschland und den Niederlanden ist seit 2007 die Produktivität um jeweils weniger als 5\% (Österreich: 5,3\%) gestiegen. Diese Länder liegen auch über den gesamten Zeitraum hinter Österreich.

\section{Abbildung 7: Entwicklung der Arbeitsproduktivität, 1998-2015 (1998 = 100)}

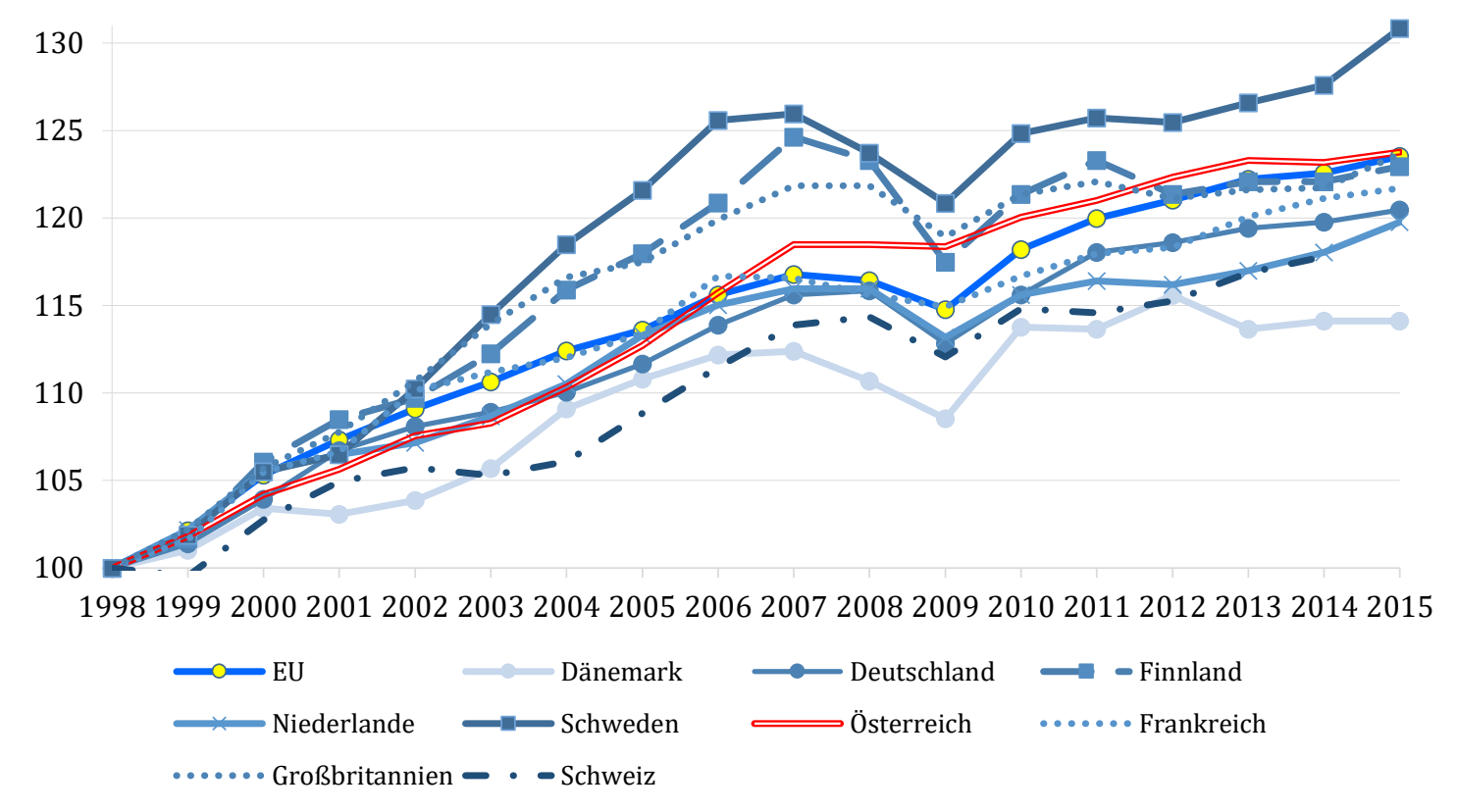

Quelle: Eurostat, eigene Berechnung und Darstellung

Die Industrialisierung hat hohe Produktivitätszuwächse gebracht, da der industrielle Sektor traditionell raschere Produktivitätsfortschritte erzielt als die Dienstleistungsbranchen. Daher kann im Ländervergleich ein höheres Produktivitätswachstum auch auf einen stärker zunehmenden (oder schwächer fallenden) Anteil des industriellen Sektors an der gesamten BWS zurückzuführen sein. Eine Untersuchung von Weyerstraß (2016) zeigt, dass Österreich in verschiedenen Industriebranchen in der Produktivität innerhalb der EU im vorderen Feld positioniert ist. In der Fahrzeugindustrie liegt Österreich hinter Deutschland und in der Metallerzeugung und -bearbeitung hinter Belgien jeweils an zweiter Stelle. Die führenden Innovationsländer sind nicht immer die produktivsten und liegen keineswegs immer vor Österreich. Abbildung 8 vergleicht Österreich mit den Innovation Leader in den nach Eurostat definierten vier Technologieklassen „niedrig“, „mittelniedrig“, „mittelhoch“ und „hoch“. Das 
Bild ist zwiespältig. Zwar liegt Österreich in der Hochtechnologie zurück, schlägt aber in den Klassen Mittelniedrig- und Mittelhoch-Technologie alle Innovation Leader. Deutschland fällt sogar in allen Klassen gegenüber Österreich zurück.

Abbildung 8: Arbeitsproduktivität nach Technologieklassen in Tsd. Euro je Beschäftigten, 2014

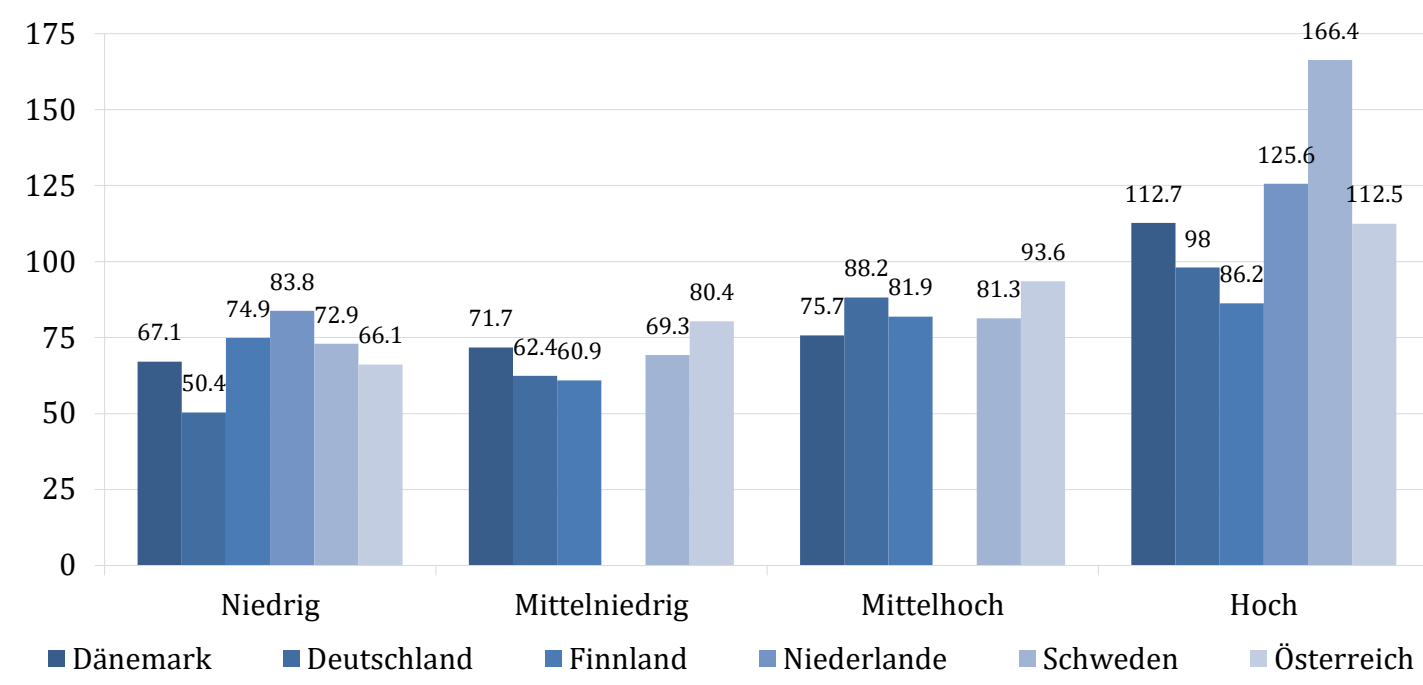

Anmerkung: Dänemark: Daten für 2008, Schweden: Daten für 2010; keine Daten für die Niederlande für die Klassen mittelniedrig und mittelhoch

Quelle: Eurostat, eigene Darstellung

Tabelle 3: Anteil innovativer Unternehmen (Rang innerhalb der EU in Klammern), 2010-2012

\begin{tabular}{llllll}
\hline & $\begin{array}{l}\text { Innovative } \\
\text { Unternehmen }\end{array}$ & $\begin{array}{l}\text { Produkt- } \\
\text { innovationen }\end{array}$ & $\begin{array}{l}\text { Prozess- } \\
\text { innovationen }\end{array}$ & $\begin{array}{l}\text { Organisator. } \\
\text { Innovationen }\end{array}$ & $\begin{array}{l}\text { Marketing- } \\
\text { innovationen }\end{array}$ \\
\hline EU-28 & $48,9 \%$ & $23,7 \%$ & $21,4 \%$ & $27,5 \%$ & $24,3 \%$ \\
\hline Dänemark & $51,1 \%(14)$ & $24,8 \%(12)$ & $22,9 \%(17)$ & $32,2 \%(9)$ & $29,5 \%(11)$ \\
Deutschland & $66,9 \%(1)$ & $35,8 \%(1)$ & $25,5(12)$ & $32,2 \%(8)$ & $34,4 \%(3)$ \\
Finnland & $52,6 \%(10)$ & $31,0 \%(5)$ & $29,3(5)$ & $29,7 \%(11)$ & $26,5 \%(13)$ \\
Niederlande & $51,4 \%(12)$ & $31,9 \%(2)$ & $25,9 \%(9)$ & $27,3 \%(13)$ & $23,2 \%(16)$ \\
Schweden & $55,9 \%(5)$ & $31,5 \%(4)$ & $23,9 \%(15)$ & $25,3 \%(16)$ & $30,4 \%(8)$ \\
\hline Österreich & $54,4 \%(8)$ & $26,6 \%(9)$ & $28,7 \%(6)$ & $36,4 \%(2)$ & $29,5 \%(9)$ \\
\hline Frankreich & $53,4(9)$ & $24,2(13)$ & $24,1(13)$ & $34,2(5)$ & $25,4(14)$ \\
Großbritannien & $50,3(15)$ & $24,0(14)$ & $14,1(21)$ & $34,2(4)$ & $16,8(23)$ \\
\hline
\end{tabular}

Quelle: Eurostat, eigene Berechnungen

Der Community Innovation Survey (CIS) der EU erhebt die Innovationstätigkeiten in den Kernbranchen der Industrie und des Dienstleistungssektors. Der Survey stuft Unternehmen als innovativ ein, wenn sie im Beobachtungszeitraum Produkt-, Prozess-, organisatorische oder Marketinginnovationen durchgeführt haben. Tabelle 3 (für die Schweiz sind keine Daten 
verfügbar) zeigt wieder, dass Österreich zwar keine Spitzenposition einnimmt, aber in allen Kategorien besser als einige oder sogar besser als alle Innovation Leader abschneidet, und in allen Kategorien vor Frankreich und Großbritannien liegt. Die Zahl der Unternehmen, die neue Produkte auf den Markt bringen, ist relativ gering. Österreich liegt EU-weit nur auf Rang neun, Deutschland besetzt den ersten Rang. Bei den Prozessinnovationen wird Österreich nur von Finnland geschlagen. Dagegen liegt es bei den organisatorischen Innovationen EU-weit sogar auf Platz zwei (hinter Luxemburg) und mit großem Abstand vor allen Innovation Leader. Sämtliche Indikatoren sehen also die Stärken eher bei Prozessinnovationen und weniger bei Produktinnovationen. Im Hinblick auf den Trend zur „Industrie 4.0", der

vorwiegend auf den Produktionsprozess abstellt, könnte hier ein Vorteil liegen. Österreich sollte die Möglichkeiten aus „Industrie 4.0“ offensiv ausschöpfen.

\subsubsection{Exporte}

Mit 53,1\% (2015, inkl. Dienstleistungen) hat Österreich eine recht hohe Exportquote, platziert sich innerhalb der EU jedoch nur im Mittelfeld. Die Zahl ist freilich mit Vorsicht zu interpretieren, da sie stark von der Größe des Landes abhängt und nicht um Vorleistungen korrigiert ist. Ebenso vorsichtig zu interpretieren sind auch die Anteile der Mittel- und Hochtechnologie-Exporte an allen Exporten. Österreich liegt hier knapp über dem EU-Schnitt; mit Ausnahme Deutschlands weisen alle Innovation Leader unterdurchschnittliche Werte auf. Spitzenreiter ist Ungarn, das im EIS nur als Moderate Innovator eingestuft wird. Auch die Slowakei und Tschechien platzieren sich deutlich vor Österreich.

Die Wettbewerbsfähigkeit einer Volkswirtschaft liegt in der Wettbewerbsfähigkeit ihrer Unternehmen begründet, welche wiederum von deren Produktivität und Größe abhängt. Nur Unternehmen, die überaus innovativ und produktiv sind, werden zu Exporteuren (Melitz, 2003, und Altomonte u.a., 2012). Um die Exportkosten zu tragen, muss ein Unternehmen wiederum eine gewisse Größe aufweisen, um die Fixkosten decken zu können. Für Österreich stellt Oberhofer (2014) fest, dass mehr als zwei Drittel aller Exporte in den EU-Raum auf nur 9\% aller Unternehmen mit mehr als 50 Beschäftigten entfallen. Bei Exporten in den NichtEU-Raum dominieren die großen Unternehmen noch mehr. Im EU-Vergleich sind Klein- und Mikro-Betriebe mit weniger als zehn Beschäftigten in Österreich überrepräsentiert. Trotz ihrer zahlenmäßigen Dominanz sind die KMU beim Export jedoch unterrepräsentiert. Die F\&E-Förderung soll Marktstörungen abbauen, indem sie für externe Innovationserträge kompensiert und Finanzierungsbeschränkungen auflockert. Da kleine KMU und Wachstumsunternehmen am meisten unter diesen Marktstörungen leiden, entfaltet die F\&EFörderung dort ihren größten Wachstumsimpuls. Damit wird ihre Wettbewerbsfähigkeit gesteigert und werden mehr von ihnen ausländische Märkte erschließen, sodass am Ende die KMU stärker im Export vertreten sein werden.

\subsubsection{Wissenschaftliche Publikationen}

Der Erfolgsindikator der Grundlagenforschung sind wissenschaftliche Publikationen. Abbildung 9 zeigt im Zeitraum 1996 bis 2014 für Österreich die absolute Zahl zusammen mit dem Anteil an den zitierfähigen Publikationen weltweit. Die wachsende Publikationsleistung in der Anzahl dürfte die gestiegenen Forschungsausgaben widerspiegeln. Allerdings schwanken die Wachstumsraten und fielen nach 2000 besonders hoch aus. So betrug das 
kumulative Wachstum zwischen 2000 und 2010 103,5\% und verdoppelte sich damit die Zahl der Publikationen. Seitdem ist das Wachstum allerdings wieder abgeflacht und betrug von 2010 bis 2014 kumulativ nur 19,7\%. Bemerkenswerterweise entwickelte sich Österreichs weltweiter Anteil nicht parallel zu den internen Wachstumsraten. Zwar gab es einen erheblichen Anstieg des „wissenschaftlichen Marktanteils“ bis 2003, als mit knapp 0,8\% ein Maximum erreicht wurde; seitdem nahm der Anteil allerdings ab und fiel bis 2014 auf nur noch 0,7\%, was dem Anteil von 1996 entspricht. Dieser Anteil ist freilich auch von globalen Entwicklungen abhängig. So hat z.B. China die Zahl seiner Publikationen von 1996 bis 2014 von 28.656 auf 470.281, also um das Sechzehnfache, gesteigert.

Die wissenschaftlichen Publikationen sind innerhalb der EU weniger ungleich verteilt als die Patente. Tabelle 4 vergleicht in Analogie zu Tabelle 2 Österreichs wissenschaftliche Publikationsleistung mit der gesamten EU und mit den Innovation Leader. Mit einem 29,5\%Anteil an allen Publikationen der EU ist die Dominanz der Innovation Leader bei weitem nicht so stark ausgeprägt wie bei den Patentanmeldungen, wo der Anteil 54,3\% beträgt. Frankreich und Großbritannien liegen hier gleichauf, bei den Zitierungen je Publikation sogar weit vor den Innovation Leader. Relativ zu ihren F\&E-Ausgaben sind die Innovation Leader ebenso wie Österreich bei den Publikationen unterrepräsentiert.

Abbildung 9: Zitierfähige wissenschaftliche Publikationen aus Österreich, 1996-2014

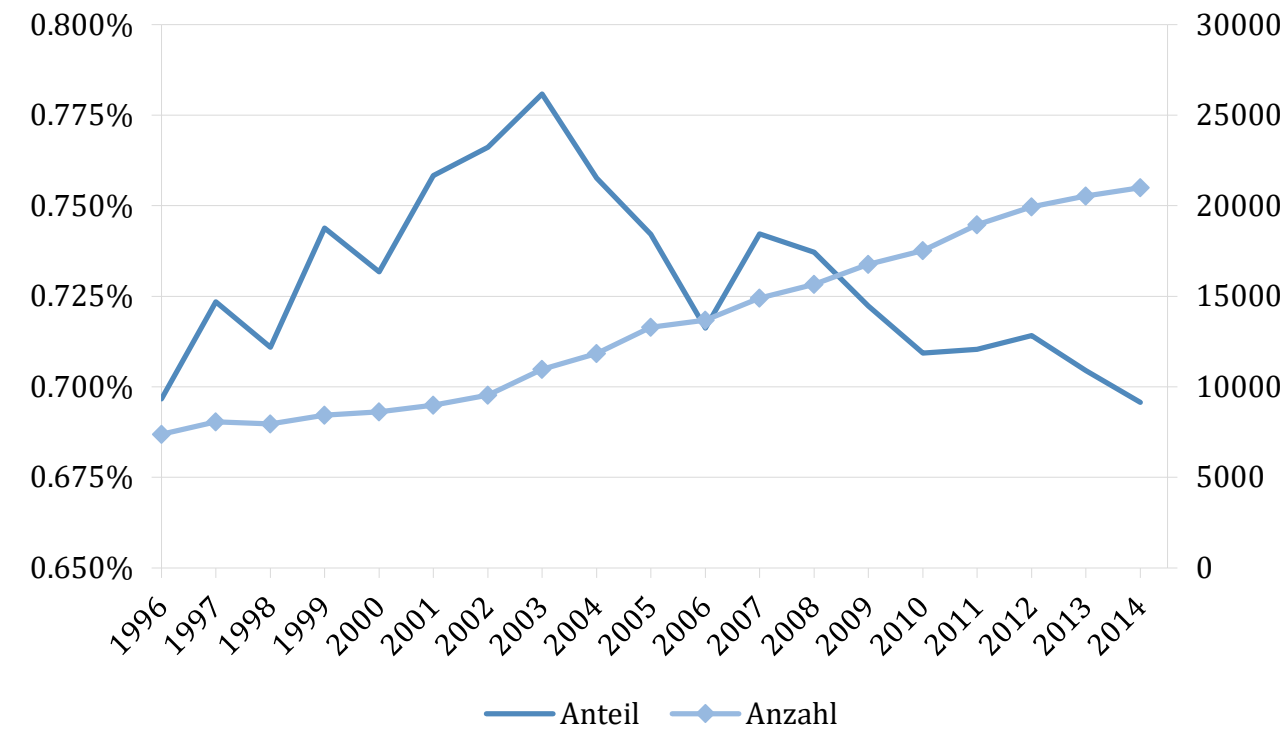

Bemerkung: Weltweiter Anteil [linke Skala] und absolute Anzahl [rechte Skala]. Quelle: Scimago Journal \& Country Rank, eigene Berechnungen und Darstellung

Der Anteil Österreichs an den Publikationen der EU ist etwas geringer als der Anteil an der BWS. Es besteht ein gewisses bislang nicht ausgeschöpftes Potenzial. Die Zahl der Zitierungen kann als Indikator für die Publikationsqualität gelten. Bei den Zitierungen je Publikation weist Österreich zwar einen Vorsprung gegenüber der EU auf, allerdings zugleich auch einen Rückstand und somit Aufholbedarf gegenüber den Innovation Leader. 
Tabelle 4: Publikationen und Zitierungen, Summen 2010-2014

\begin{tabular}{lllll}
\hline & $\begin{array}{l}\text { Publikationen } \\
{[\text { in 1.000] }}\end{array}$ & $\begin{array}{l}\text { Zitierfähige } \\
\text { Publ. [in 1.000] }\end{array}$ & $\begin{array}{l}\text { Zitierungen } \\
\text { [in 1.000] }\end{array}$ & $\begin{array}{l}\text { Zitierungen je } \\
\text { Publikation }\end{array}$ \\
\hline EU & $4.797,98$ & $4.313,13$ & $39.665,26$ & 8,267 \\
Innovation Leader & $1.417,02$ & $1.286,31$ & $13.342,18$ & 9,416 \\
Österreich & 108.11 & 97,95 & 974,97 & 9,018 \\
\hline Innovation Leader/EU & $29,53 \%$ & $29,82 \%$ & $33,64 \%$ & $113,89 \%$ \\
Österreich/EU & $2,25 \%$ & $2,27 \%$ & $2,46 \%$ & $109,09 \%$ \\
Österreich/Innovation Leader & $7,63 \%$ & $7,62 \%$ & $7,31 \%$ & $95,78 \%$ \\
\hline Großbritannien & 887,64 & 735,10 & 7711,29 & 8,687 \\
Frankreich & 561,11 & 515,13 & 4518,23 & 8,052 \\
Schweiz & 195,35 & 177,30 & 2276,33 & 11,652 \\
\hline (GB \& F)/EU & $30,19 \%$ & $28,99 \%$ & $30,83 \%$ & $202,49 \%$ \\
Österreich/Schweiz & $55,34 \%$ & $55,25 \%$ & $42,83 \%$ & $77,39 \%$ \\
\hline
\end{tabular}

Anmerkung: Innovation Leader sind Dänemark, Deutschland, Finnland, Niederlande und Schweden. Quelle: Scimago Journal \& Country Rank, eigene Berechnungen

\subsection{Finanzierung}

Österreich ist in der Lage, erhebliche Ressourcen insbesondere für die unternehmerische F\&E aufzubringen. Dabei spielt der Staat eine wichtigere Rolle als in vergleichbaren Ländern. Ohne Zweifel ist die dynamische Entwicklung der Innovationsaktivitäten in Österreich ohne die „sichtbare Hand“ des Staates schwer zu erklären. Nicht zuletzt hat der stetige Ausbau der indirekten, steuerlichen Forschungsförderung für Unternehmen dazu beigetragen, die seit 2016 eine Förderhöhe von 12\% bietet. Die private Initiative scheint dagegen nachzuhinken. Damit einhergehend stellt sich daher auch die Frage nach dem richtigen Verhältnis in der Förderung der privaten F\&E und der staatlichen Grundlagenforschung.

\subsubsection{Staatliche und privatwirtschaftliche Finanzierung}

Die gesamten Forschungsausgaben in Österreich beliefen sich in 2013 auf insgesamt 9,57 Mrd. Euro, wovon 70,8\% im Unternehmenssektor und 24,4\% im Hochschulsektor getätigt wurden. Die Anteile der Sektoren variieren, haben sich aber über die letzten zehn Jahre nicht wesentlich geändert.4 Bei der Finanzierung ist der Anteil ausländischer Quellen zwar leicht zurückgegangen, im Großen und Ganzen haben sich jedoch auch hier die Finanzierungsanteile in den letzten zehn Jahren kaum verändert.5

4 Die Anteile betrugen für die Jahre 2004/2007/2011/2013 im Hochschulsektor 26,7\%/23,8\%/25,6\%/24,3\%, im Sektor Staat $5,1 \% / 5,3 \% / 5,1 \% / 4,4 \%$, im privaten gemeinnützigen Sektor $0,4 \% / 0,3 \% / 0,5 \% / 0,4 \%$ und im Unternehmenssektor 67,8\%/70,6\%/68,8\%/70,9\% (Quelle: Statistik Austria).

5 Die Anteile betrugen für die Jahre 2004/2007/2011/2013 für den öffentlichen Sektor 33,0\%/32,9\%/36,4\%/34,2\%, für den Unternehmenssektor 47,1\%/48,7\%/46,2\%/48,7\%, für den privaten gemeinnützigen Sektor jeweils 0,5\%, für das Ausland ohne EU 17,7\%/16,4\%/15,1\%/14,7\%, für die EU 1,7\%/1,5\%/1,8\%/1,9\% (Quelle: Statistik Austria). 


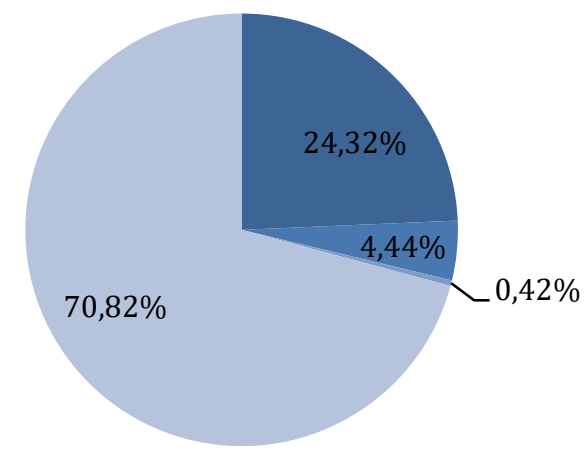

- Hochschulsektor

Sektor Staat

- Privater gemeinnütziger Sektor

- Unternehmenssektor

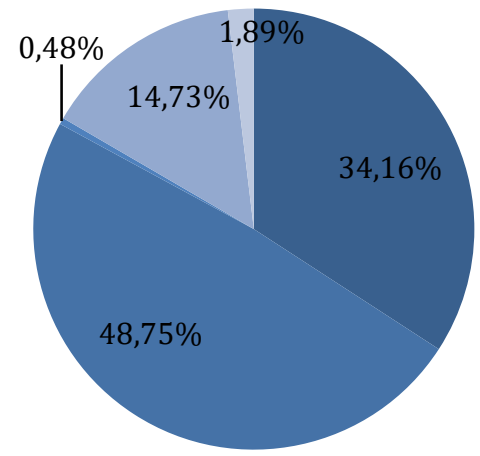

- Öffentlicher Sektor

- Unternehmenssektor

- Privater gemeinnütziger Sektor

- Ausland ohne EU

$\square \mathrm{EU}$

Quelle: Statistik Austria, eigene Berechnungen Anreize und Förderungen

Im Jahr 2013 finanzierten der Staat 34,2\%, die Unternehmen 48,7\%, das Ausland ohne EU 14,7\% und die EU 1,9\%. Der allergrößte Teil aus dem „Ausland ohne EU“ stammt dabei von ausländischen Unternehmen auch aus der EU, sodass der Unternehmenssektor insgesamt mit einem Finanzierungsanteil von 63,5\% den Löwenanteil beisteuert. Im internationalen Vergleich ist die Finanzierung durch ausländische Unternehmen relativ hoch und trägt diese $23,2 \%(=14,7 / 63,5)$ der Finanzierung des Unternehmenssektors. Trotzdem erreicht der Finanzierungsanteil der Unternehmen an den gesamten Forschungsausgaben immer noch nicht die Zielvorgaben. Der von der EU für das Jahr 2020 angestrebte Wert beträgt 66\%. Dasselbe Ziel hat auch die FTI-Strategie des Bundes von 2011 gesetzt. Abbildung 10 zeigt zudem, dass zwischen der von Unternehmen durchgeführten und der von Unternehmen finanzierten F\&E eine Lücke von 7,4 Prozentpunkte klafft, was etwa 10,4\% der von den Unternehmen durchgeführten F\&E entspricht. Mit anderen Worten wird ein wesentlicher Teil der unternehmerischen Forschungsinvestitionen staatlich subventioniert.

Österreich verfügt über ein Portfolio von Förderprogrammen, welche die F\&E-Aktivitäten der Unternehmen und ihre Kooperation mit der Wissenschaft unterstützen. Die Förderungen erfassen nach Abbildung 11 ca. 40\% aller Unternehmen mit Produkt- und/oder Prozessinnovationen. Im OECD-Vergleich liegt Österreich damit an sechster Stelle und, mit Ausnahme der Niederlande, vor allen im Survey erfassten Innovation-Leader. Vor allem Deutschland und die Schweiz sind mit Förderungen deutlich knapper. Der Anteil an unterstützten Großunternehmen ist in Deutschland nur halb so groß wie in Österreich. In der Schweiz werden nur etwa 23\% der Großunternehmen mit öffentlichen Geldern unterstützt, in Österreich sind es 66\%. Österreich unterstützt anteilsmäßig etwa gleich viele Großunternehmen wie die Niederlande, aber um 9 Prozentpunkte weniger KMU. Der Anteil geförderter Großunternehmen ist in Österreich um ca. 78\% höher als der KMU-Anteil. 
Abbildung 11: Geförderte in \% aller Unternehmen mit Produkt- und/oder Prozessinnovationen

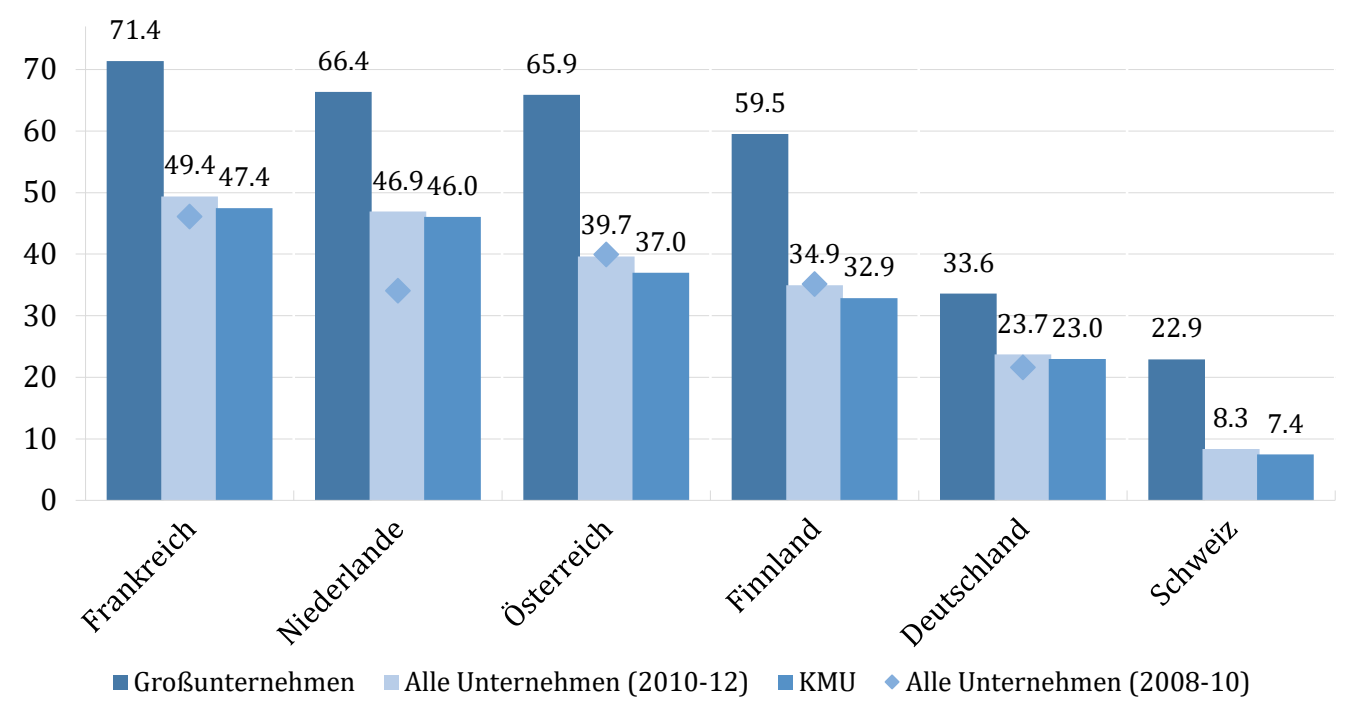

Quelle: OECD (2015) basierend auf CIS 2010 und CIS 2012, eigene Darstellung

Dem hohen Anteil an geförderten Unternehmen stehen auf der anderen Seite hohe fiskalische Kosten gegenüber. Abbildung 12 zeigt die Ausgaben für direkte und indirekte F\&E-Förderung in \% des BIP für das Jahr 2013. Auch hier nimmt Österreich nach Frankreich einen Spitzenplatz ein und liegt vor allen Innovation Leader, und gibt in Prozent des BIP 13-mal mehr aus als die Schweiz, und um 60\% mehr als der nächstplatzierte Innovation Leader, die Niederlande.

Abbildung 12: Direkte und indirekte F\&E-Fördermaßnahmen in \% des BIP, 2013

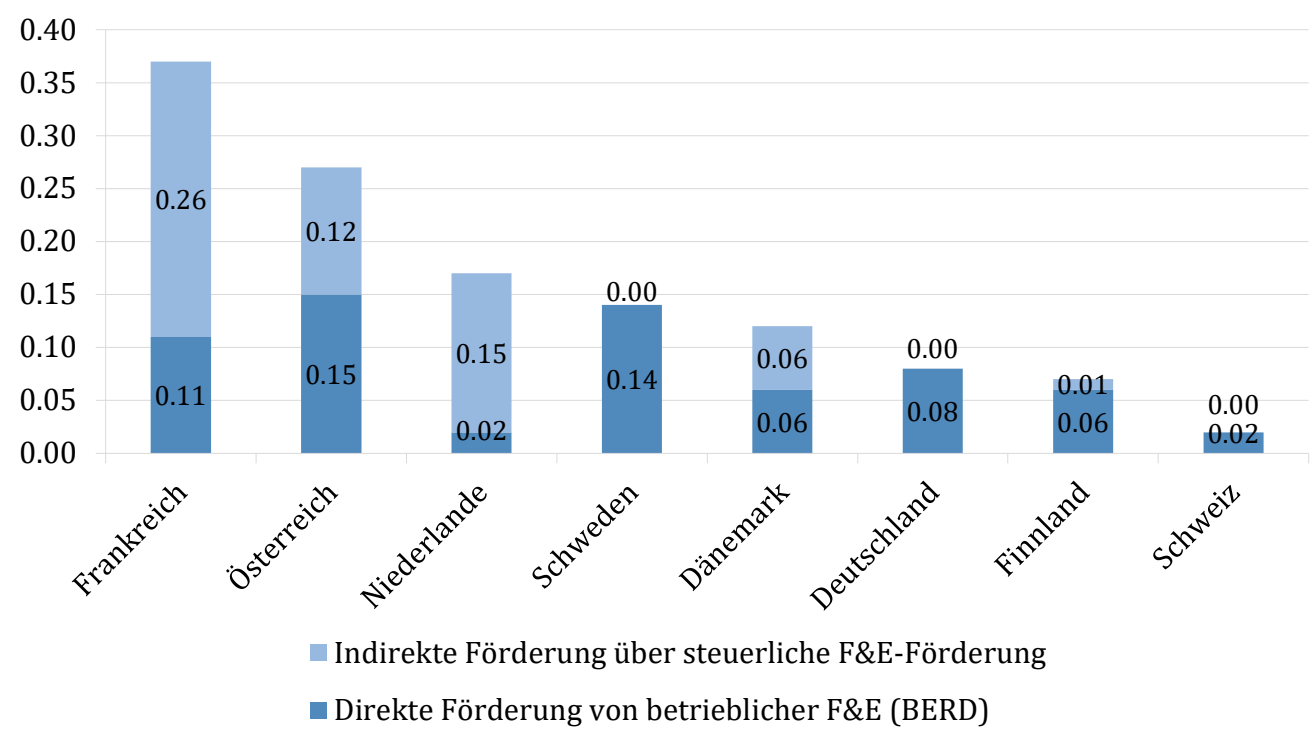

Quelle: OECD 2015, eigene Darstellung 
Das Verhältnis zwischen direkter und indirekter Förderung ist im Vergleich zu den anderen Ländern ausgewogen. Die indirekte Forschungsförderung in Österreich, die Forschungsprämie, besteht aus einer steuerlichen Gutschrift für die F\&E-Aufwendungen der Unternehmen. Das gesamte Volumen der Forschungsprämie betrug in 2013 467,7 Mio. Euro. Im Gegensatz zur direkten Förderung steht die Steuergutschrift allen Unternehmen zu und ist neutral hinsichtlich des Inhalts und des Charakters der F\&E-Projekte. Diese niedrige Zutrittsschwelle ist ein besonderer Vorteil gerade auch für die KMU.

\subsubsection{Drittmittelfinanzierung an den Universitäten}

Der Hochschulsektor in Österreich steuert etwa ein Viertel der gesamten F\&E bei, das sind 0,73\% des BIP im Jahr 2014. Damit überholte Österreich bereits in 2008 den neuen Innovation Leader, die Niederlande, liegt abwechselnd knapp vor und hinter Finnland und reiht sich heute innerhalb der EU an dritter Stelle hinter Dänemark $(1,01 \%)$ und Schweden (0,92\%) ein. Der Anteil des Staates an der universitären F\&E hat von 1998 bis 2013 von 95,2\% auf $85,4 \%$ abgenommen.

Tabelle 5: Finanzierung der universitären F\&E, 2013

\begin{tabular}{llllllll}
\hline & Staat & $\begin{array}{l}\text { Hochschul } \\
\text {-sektor }\end{array}$ & $\begin{array}{l}\text { inländ. } \\
\text { Unter- } \\
\text { nehmen }\end{array}$ & $\begin{array}{l}\text { ausländ. } \\
\text { Unter- } \\
\text { nehmen }\end{array}$ & $\begin{array}{l}\text { private } \\
\text { Organisa } \\
\text {-tionen }\end{array}$ & $\begin{array}{l}\text { EU- } \\
\text { Kommis- } \\
\text { sion }\end{array}$ & $\begin{array}{l}\text { sonstige } \\
\text { ausländ. } \\
\text { Mittel }\end{array}$ \\
\hline Dänemark & $79,1 \%$ & $\mathrm{k}, \mathrm{A}$, & $2,5 \%$ & $1,0 \%$ & $10,7 \%$ & $4,4 \%$ & $2,3 \%$ \\
Deutschland & $80,6 \%$ & $\mathrm{k}, \mathrm{A}$, & $14,0 \%$ & $\mathrm{k}, \mathrm{A}$, & $\mathrm{k}, \mathrm{A}$, & $5,2 \%$ & $0,2 \%$ \\
Finnland & $80,0 \%$ & $1,1 \%$ & $5,0 \%$ & $1,2 \%$ & $3,9 \%$ & $7,6 \%$ & $1,2 \%$ \\
Niederlande & $77,1 \%$ & $0,0 \%$ & $7,6 \%$ & $\mathrm{k}, \mathrm{A}$, & $7,6 \%$ & $\mathrm{k}, \mathrm{A}$, & $7,8 \%$ \\
Schweden & $76,2 \%$ & $3,2 \%$ & $3,8 \%$ & $0,8 \%$ & $10,3 \%$ & $4,3 \%$ & $1,4 \%$ \\
\hline Österreich & $85,4 \%$ & $2,4 \%$ & $5,1 \%$ & $1,1 \%$ & $1,2 \%$ & $3,9 \%$ & $0,9 \%$ \\
\hline Frankreich & $88,3 \%$ & $4,0 \%$ & $2,8 \%$ & $0,5 \%$ & $1,2 \%$ & $2,6 \%$ & $0,5 \%$ \\
Großbrit. & $62,9 \%$ & $3,9 \%$ & $4,1 \%$ & $1,9 \%$ & $13,8 \%$ & $7,5 \%$ & $5,9 \%$ \\
Schweiz & $80,0 \%$ & $4,0 \%$ & $10,9 \%$ & $\mathrm{k}, \mathrm{A} \%$ & $0,2 \%$ & $0,0 \%$ & $4,8 \%$ \\
\hline
\end{tabular}

Anmerkung: Zellen ohne Angabe (k.A.) ergeben sich aus abweichenden Definitionen, die Zeilensummen ergeben jeweils $100 \%$.

Quelle: Eurostat, eigene Berechnungen

Tabelle 5 vergleicht die Finanzierung universitärer F\&E Österreichs mit jener der Innovation Leader. Obwohl sich die EU-Mittel seit 2004 mit einer Steigerung von 96,1\% nominell nahezu verdoppelt haben, sind sie immer noch auffallend niedrig. Der Anteil der unternehmensfinanzierten F\&E ist in Österreich deutlich niedriger als in Deutschland und auch in den Niederlanden, aber gleichauf mit Finnland. In Großbritannien trägt der Staat mit $62,9 \%$ besonders wenig zur Finanzierung bei, umso mehr jedoch die privaten Organisationen. Auch in der Schweiz finanzieren Unternehmen mehr als das Doppelte als in Österreich. Die Möglichkeiten der Drittmittelfinanzierung dürften somit offenbar erheblich von der Präsenz multinationaler Unternehmen abhängen, die in Österreich vergleichsweise unterrepräsentiert sind. Die heimischen Unternehmen haben ihren Finanzierungsanteil von 1998 bis 2007 mit einem Anstieg von 1,75\% auf 5,74\% mehr als verdreifacht, in nominellen 
Geldeinheiten sogar mehr als verfünffacht. Seit 2007 stagnierte das Engagement, sodass der Unternehmensanteil bis 2013 wieder auf 5,1\% zurückfiel (vgl. Tabelle 5).

Die Drittmittelforschung spielt eine immer wichtigere Rolle. Der Wettbewerb verschärft sich und der Aufwand für die Einwerbung von Drittmitteln nimmt zu. Während die Globalfinanzierung nur mehr 73,4\% der universitären Budgets ausmachte, stammten im Jahr 2014 bereits 16,1\% aus Drittmitteln.6 Die heimischen Unternehmen steuern dabei nur etwa ein Viertel bei (BMWFW, 2016).

\subsubsection{Finanzierungsbeitrag ausländischer Unternehmen}

Darstellungen wie Abbildung 10 sind insofern irreführend, als die Kategorie „Ausland ohne EU“ nur aus dem Ausland stammende Finanzierung umfasst. 7 Nicht darin enthalten sind F\&EInvestitionen ohne direkte Finanzierung aus dem Ausland, die von in Österreich ansässigen, aber ausländisch kontrollierten Unternehmen durchgeführt werden. Berücksichtigt man auch diese Ausgaben - gleichgültig, ob die ausländische Mutter nun tatsächlich die F\&E in Österreich finanziert oder nicht - so fällt der Anteil ausländischer F\&E-Finanzierung in Österreich wesentlich höher aus. Rund die Hälfte der unternehmerischen F\&E-Investitionen werden nach dieser Methode dem Ausland zugeschrieben. Dieser Anteil hat während der 2000er-Jahre erheblich zugenommen und ist im Vergleich zu anderen EU- und OECD-Staaten hoch (Dachs u.a., 2013 und 2015). Trotz Internationalisierung stammen die Mittel der ausländisch finanzierten unternehmerischen F\&E mit etwa 60\% überwiegend aus Deutschland und der Schweiz und weitere 15\% aus den USA. Dabei ist der Anteil des Dienstleistungssektors in den letzten Jahren erheblich gestiegen und machte in 2013 bereits ein Drittel aus. Der weitaus größte Anteil entfällt auch hier auf die Mittelhoch- und Hochtechnologiebranchen des industriellen Sektors (Dachs u.a., 2015).

Mehrere Studien (Sieber, 2008; Berger u.a., 2011; BMWF u.a., 2010; Pöchacker Innovation Consulting und IMAS International, 2013) haben die Motive internationaler Konzerne für F\&E in Österreich untersucht. Sie stützen sich auf Befragungen mit gewissen methodischen Problemen (niedrige Rücklaufquoten, niedrige Fallzahlen). Dennoch kristallisieren sich nach Leitner u.a. (2015) wichtige Motive gereiht nach abnehmender Bedeutung heraus: (i) Erfolg heimischer Töchter bei konzerninternen Ausschreibungen von F\&E-Projekten; (ii) Verfügbarkeit qualifizierter Arbeitskräfte und wissenschaftlichen Personals; (iii) gute Kooperationsbedingungen mit Universitäten und sonstigen Forschungseinrichtungen; (iv) günstige Förderbedingungen sowie (v) Nähe zu Absatzmärkten. Offensichtlich zählen in erster Linie die Wettbewerbsfähigkeit österreichischer F\&E und danach die Leistungen des Staates. Die Verfügbarkeit von qualifiziertem Humankapital ist besonders bedeutend. Tatsächlich zeigen neuere Daten (Dachs, 2016), dass knapp 40\% des F\&E-Personals im Unternehmenssektor bei ausländischen Firmen angestellt sind. Neben dem bloßen Finanzierungsbeitrag zeitigt die Forschung ausländischer Unternehmen weitere Vorteile für

6 Außerdem: Studienbeiträge 6,1\%, Einnahmen aus universitärer Weiterbildung 1,0\%, sonstige Erlöse und Kostenersätze 3,4\%; Daten nach BMWFW (2016).

7 Darin enthalten sind Mittel von ausländischen verbundenen Unternehmen (d.h. Unternehmen, die zum selben Konzern wie das F\&E betreibende Unternehmen gehören), Mittel von anderen ausländischen Unternehmen sowie sonstige Finanzierung aus dem Ausland (d.h. von ausländischen öffentlichen sowie privaten gemeinnützigen Einrichtungen). 
die heimische Wirtschaft wie z.B. die Übertragung von technologischem Wissen und Knowhow der Muttergesellschaften auf das Gastland.

\subsubsection{Unternehmensgründungen und Wagniskapital}

Wagniskapital kann die Entwicklung junger, innovativer Firmen stark beflügeln (siehe auch Abschnitt 4.5). Ein Grund ist die mangelnde Kreditwürdigkeit junger Unternehmen, weshalb die Finanzierung über Banken oft nicht möglich ist. Diese Lücke schließt das Wagniskapital. Viele der Firmen verfügen über hohes Wachstumspotenzial und sind daher für risikofreudige Investoren interessant. In den USA erzielen die Wagnisfinanziers rund 85\% aller Profite aus nur 10\% aller Investitionen (Ewens und Rhodes-Kropf, 2015). Das überaus hohe Risiko wird mit den enormen Gewinnen entgolten, welche die wenigen überlebenden Start-ups beisteuern. Wagnisfinanzierte Firmen wachsen wesentlich schneller als andere. In den USA werden nur etwa 1.000 der 600.000 jährlich neu gegründeten Unternehmen mit Wagniskapital finanziert, diese machen aber mehr als die Hälfte aller Börseneinführungen aus (Nanda und Rhodes-Kropf, 2016). Wagnisfinanzierung ist eine chronische Schwäche des heimischen Innovationssystems. Österreich liegt beim Verhältnis Wagniskapital/BIP in der EU abgeschlagen an 16. Stelle (Europäische Kommission, 2016). Allerdings nimmt die Quote immerhin überdurchschnittlich stark zu, mehr als bei allen Innovation Leader.

Innovative Unternehmensgründungen spielen für das Wachstum (und für die Nachfrage nach Wagniskapital) eine große Rolle. Bei der Einfachheit, Geschäfte zu betreiben („ease of doing business"), sieht die Weltbank Österreich nur im 21. von 89 Rängen. Zu einer ähnlichen Einschätzung kommt die Reihung des Weltwirtschaftsforums (Schwab, 2015) nach der Wettbewerbsfähigkeit, wo Österreich im vorderen Mittelfeld den 23. Rang belegt. Mit Ausnahme der Niederlande schneiden alle Innovation Leader besser ab. In der Einfachheit, ein Unternehmen zu gründen, liegt Österreich weit abgeschlagen auf dem 106. Rang. Beim Zugang zu Krediten sieht die Weltbank Österreich nur an 59. Stelle. Ähnlich ungünstig schätzt das Weltwirtschaftsforum die Verfügbarkeit von Wagniskapital ein (58. Rang). 


\section{$3 \quad$ F\&E, Unternehmenswachstum und Internationalisierung}

F\&E treibt die Unternehmensentwicklung und die industrielle Dynamik. Das betont die neuere theoretische Forschung (z.B. Melitz und Redding, 2012) und bestätigen die Ergebnisse der darauf aufbauenden mikroökonometrischen Untersuchungen (Altomonte u.a., 2011). Vor allem die Internationalisierung der Firmen hängt stark von den Innovationsaktivitäten und dem Produktivitätsniveau ab (vgl. z.B. Syverson, 2011; Cassiman und Golovko, 2011). Exportunternehmen und multinationale Firmen sind produktiver, innovativer und größer als die auf den Inlandsmarkt orientierten Unternehmen. Ein kleiner und sehr wettbewerbsfähiger Anteil innovativer und international orientierter Unternehmen dominiert die handelbaren Branchen und folglich die gesamte Wirtschaft.

Der österreichische Absatzmarkt gemessen am BIP macht etwa 1\% der Welt und 5\% der EU aus. Der Zugang zum Weltmarkt erschließt damit den innovativen Unternehmen weit größere Wachstumschancen, als eine Beschränkung auf den Inlandsmarkt je erlauben würde. Viele innovative und hoch spezialisierte Hidden Champions erzielen oft 80 bis $100 \%$ des Umsatzes im Ausland. Dabei treffen sie nicht nur auf heimische Konkurrenten, sondern müssen sich gegenüber der weltweiten Konkurrenz behaupten. Daher müssen sie auch mit permanenter Innovation ihren Qualitäts- und Kostenvorsprung erhalten und ihre Wettbewerbsvorteile verteidigen. Die Internationalisierung verstärkt somit wiederum die F\&E-Aktivitäten, wie Abbildung 13 veranschaulicht.

Abbildung 13: Innovation, Unternehmenswachstum und Internationalisierung

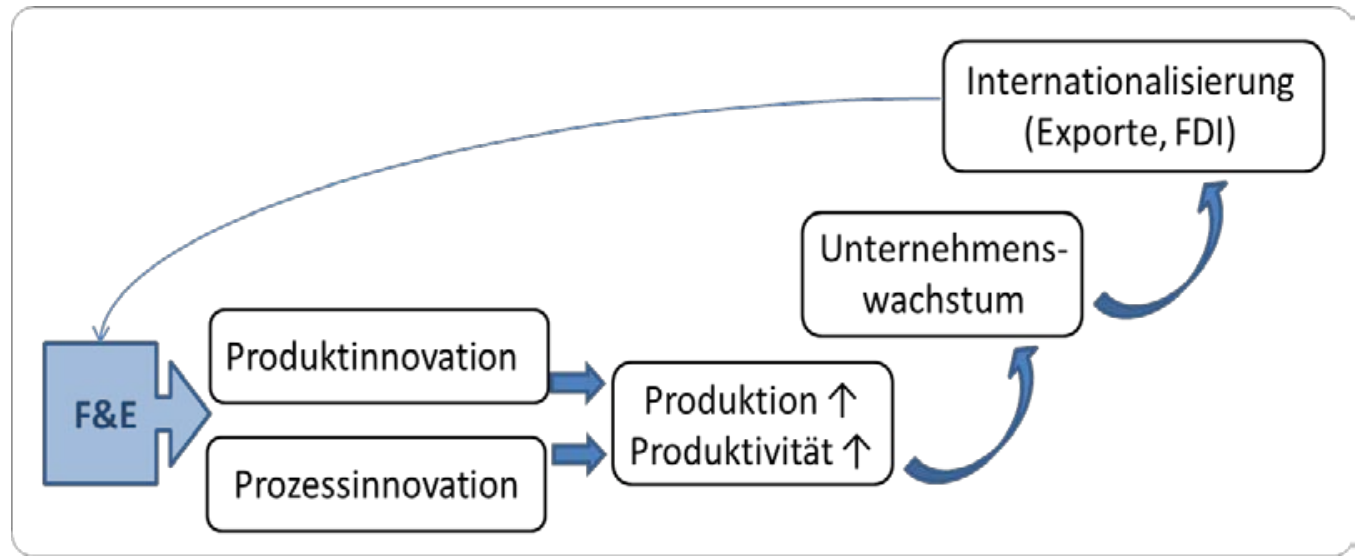

Quelle: eigene Darstellung

Das aktuelle Kapitel folgt dieser Logik. Abschnitt 3.1 zeigt, wie F\&E auf Produktion und Produktivität wirkt, und geht auf Industrie 4.0 ein. Abschnitt 3.2 widmet sich dem F\&Einduzierten Unternehmenswachstum. Danach wendet sich Abschnitt 3.3 der Internationalisierung mittels Exporten und Direktinvestitionen und der Standortfrage zu. 


\subsection{Produktivitätsentwicklung in der Industrie}

\subsubsection{Trend zur De-Industrialisierung?}

Die österreichische Industrie zeichnet sich vor allem in den mittleren Technologiesegmenten durch eine hohe Produktivität und Wettbewerbsfähigkeit aus. Die Exportqualität der heimischen technologieorientierten Industriebetriebes liegt gleichauf mit jener der Innovation Leader (RFTE, 2016). Das ist an sich eine gute Voraussetzung auch im Hinblick auf das Ziel der EU, den Anteil der Industrie an der Gesamtproduktion (gemessen am BIP) auf $20 \%$ zu erhöhen.

Abbildung 14: Anteil Industrie an Bruttowertschöpfung und Arbeitsinput in Stunden, 2015

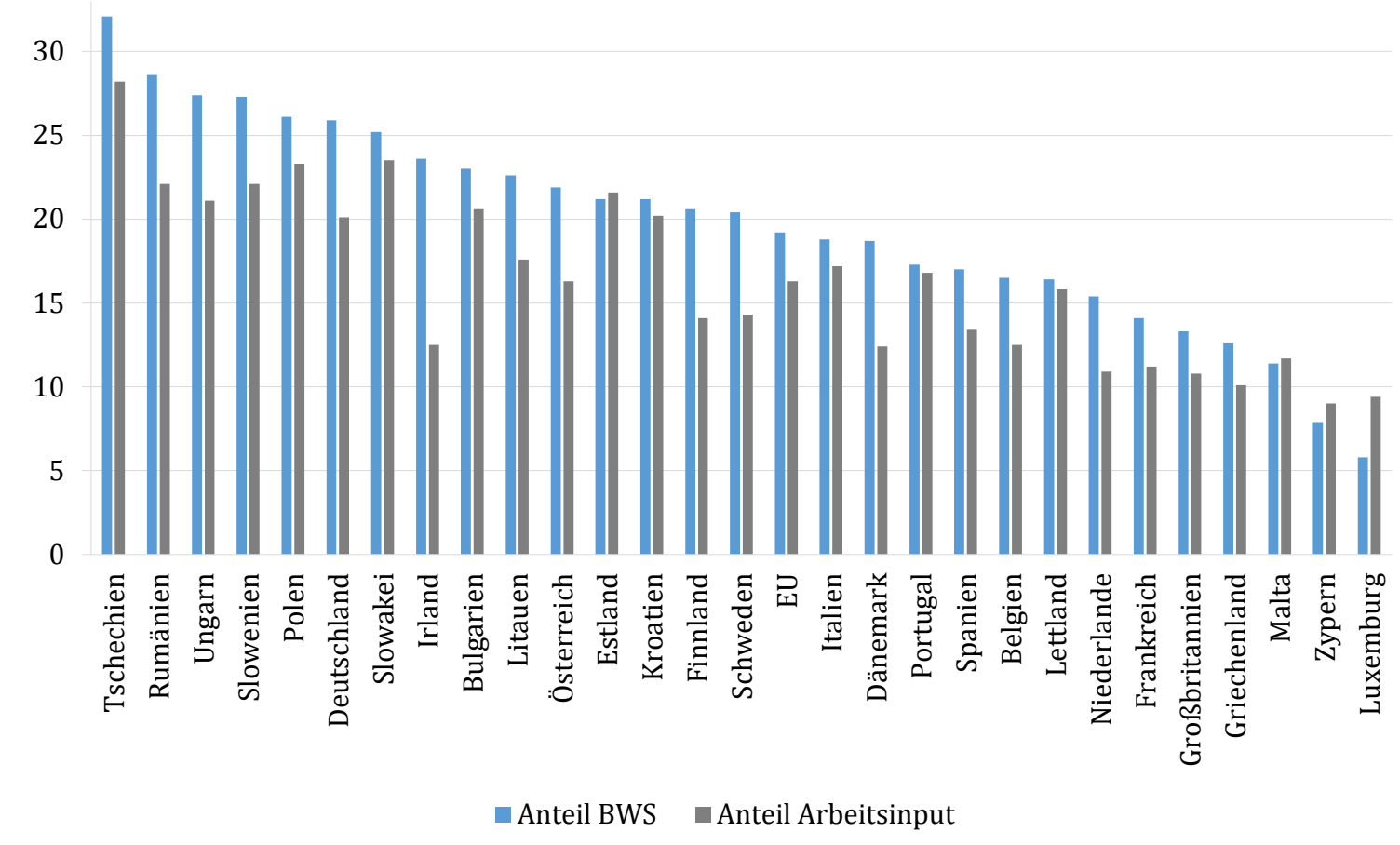

Anmerkung: Industrie ohne Baugewerbe. Belgien: 2014, Rumänien: 2013;

Quelle: Eurostat, eigene Berechnungen und Darstellung

Abbildung 14 vergleicht die Anteile der Industrie an der Bruttowertschöpfung (BWS) und an den geleisteten Arbeitsstunden in der EU.9 Unter den ersten zehn Ländern mit der höchsten industriellen BWS finden sich neben Deutschland und Irland nur neue Mitgliedstaaten (NMS). Österreich liegt mit dem elften Platz deutlich über dem EU-Schnitt und befindet sich damit in

s Die Exportqualität berechnet sich als Quotient aus den Exporten der technologieorientierten Sachgüterindustrie im höchsten und mittleren Preissegment und den Gesamtexporten der technologieorientierten Sachgüterindustrie.

9 Das BIP inkludiert im Gegensatz zur BWS indirekte Steuern und Subventionen. In Bezug auf die tatsächliche Bedeutung verschiedener Branchen und Sektoren für die Gesamtwirtschaft stellt die BWS daher die besser geeignete Bezugsgröße dar. Hinzu kommt, dass indirekte Steuern und Subventionen in verschiedenen Ländern naturgemäß variieren, weshalb das BIP als Bezugsgröße zu verzerrten Darstellungen führt. 
guter Gesellschaft zu den Innovation Leader. Unter den EU15-Staaten weisen sonst nur noch Finnland und Schweden überdurchschnittliche Anteile auf. Allerdings ist im Vergleich zu den Innovation Leader die Gründungsdynamik im österreichischen Industriesektor niedriger. Nachdem ein ähnlicher Befund auch für den Dienstleistungssektor gilt, liegt der Schluss nahe, dass die gründungsrelevanten Rahmenbedingungen in Österreich nach wie vor eine verhaltene Gründungsdynamik zur Folge haben (RFTE 2016).

Abbildung 15 veranschaulicht den sinkenden Anteil der Industrie an der BWS in Österreich und in anderen Regionen. Die „De-Industrialisierung“ der EU scheint vor allem in jenen etablierten Mitgliedstaaten stattzufinden, die nicht zu den Innovation Leader zählen. Im Unterschied zu diesen als „EU9“ bezeichneten Ländern konnten die Innovation Leader, die NMS und Österreich ihre Industrieanteile im Großen und Ganzen halten. Der Rückgang der Industrie passiert also vor allem in den etablierten Industriestaaten Westeuropas. Tatsächlich weisen Großbritannien und Frankreich hinter den Kleinstaaten Luxemburg, Malta und Zypern sowie Griechenland mittlerweile die niedrigsten Industrieanteile in der EU auf.

Abbildung 15: Anteil Industrie (ohne Baugewerbe) an der Bruttowertschöpfung, 1995-2015

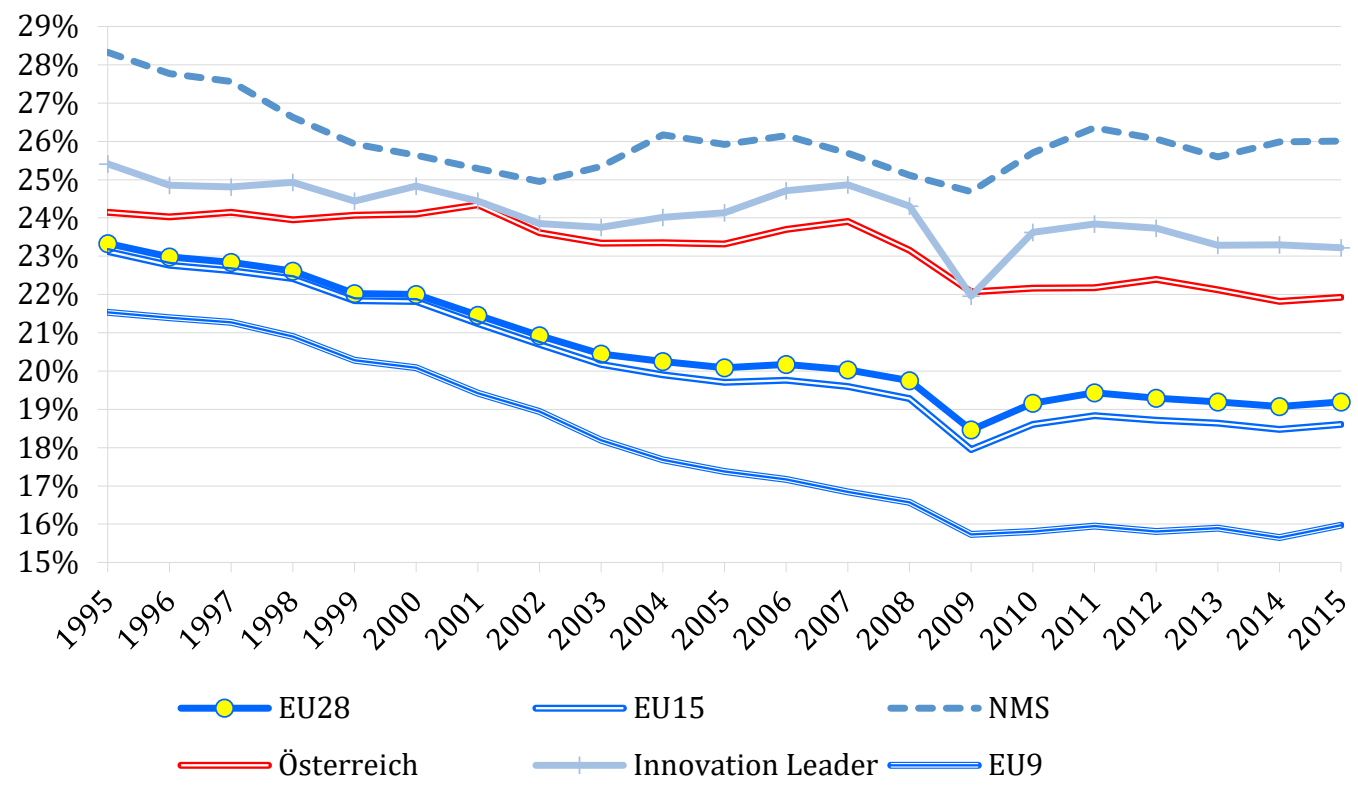

Anmerkung: Industrie ohne Baugewerbe; EU9 ist EU15 ohne Innovation Leader und Österreich Quelle: Eurostat, eigene Berechnungen und Darstellung

Die stetig steigende Produktivität der Industrie relativ zu anderen Branchen hat zwangsläufig eine Abnahme der Beschäftigung im industriellen Sektor in Österreich und anderswo zur Folge. Wenn sich F\&E, Innovation und Produktivitätssteigerungen z.B. in Form zunehmender Automatisierung überdurchschnittlich stark auf die Industrie konzentrieren, dann steigt die industrielle Produktivität wesentlich rascher als in der gesamten Wirtschaft. Bleibt die Nachfrage nach Industriegütern als Anteil am Gesamteinkommen konstant, dann benötigt die Industrie immer weniger Beschäftigte, um die Nachfrage zu befriedigen, während die BWS weniger stark betroffen ist. Die Anteile der Industrie an der BWS und Beschäftigung sollten 
unterschiedlich stark reagieren. Gerade die produktivsten und innovativsten Länder haben in Abbildung 14 tendenziell die geringsten Beschäftigungsanteile in der Industrie, wenn nicht andere Faktoren wie z.B. überdurchschnittliche Industrieexporte den Rückgang aufhalten.

\subsubsection{Produktivität und Industrieanteil}

Die Industrialisierung war historisch ein Treiber des Produktivitätswachstums. Die Industrie ist geradezu zur Basis einer breiten Mittelschicht in modernen Gesellschaften geworden.10 In vielen Ländern scheint sich der Prozess jedoch umzukehren und die Bedeutung der Industrie abzunehmen. Bis zum Ausbruch der Krise schien der Rückgang des industriellen Sektors in den etablierten Industriestaaten Frankreich, Großbritannien und den USA keine besonderen Probleme zu verursachen. Als Ausgleich nahmen Anteil und Produktionswert der Hochtechnologie-Industrie sowie wissensintensiver Dienstleistungen stetig zu. In der Krise erwiesen sich jedoch Länder mit hohem Industrieanteil deutlich widerstandsfähiger (Reiner, 2012). Industriegüter können offenbar eher exportiert werden als Dienstleistungen und damit Schwankungen der heimischen Nachfrage und ein höheres Produktivitätswachstum in der Industrie durch Exporte leichter ausgleichen.

Abbildung 16: Anteil Industrie an Bruttowertschöpfung und Produktivitätswachstum, EU15-Staaten

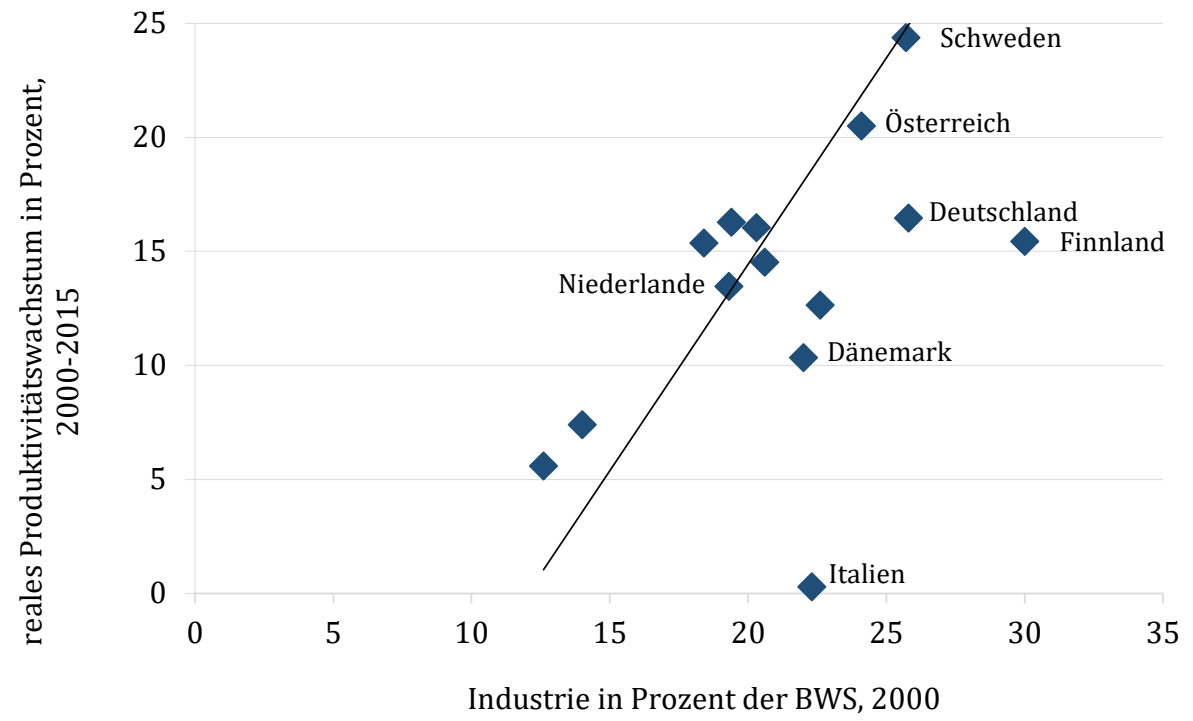

Anmerkung: Industrie ohne Baugewerbe; Belgien: 1999-2014

Quelle: Eurostat, eigene Berechnungen und Darstellung

Ein hoher Industrieanteil scheint ein hohes Produktivitätswachstum zu begünstigen. Nach Abbildung 16 gibt es innerhalb der EU tatsächlich einen positiven Zusammenhang zwischen dem industriellen Anteil an der BWS und dem Produktivitätswachstum. Für 2000 bis 2015 und für die Länder mit einem kumulierten Produktivitätswachstum von weniger als 30\% beträgt der Korrelationskoeffizient 0,42. Mit Ausnahme der Kleinstaaten Malta und Zypern

10 Vgl. dazu Dani Rodrik www.project-syndicate.org/commentary/the-manufacturing-imperative?barrier=true, abgefragt am 20.9.2016, sowie Rodrik (2015). 
konnten alle NMS ihre Produktivität kumuliert um mehr als 30\% steigern. Abgesehen von Irland konnte jedoch kein EU15-Staat ein solch hohes Produktivitätswachstum erzielen. Es liegt also ein Aufholprozess der NMS vor, der den Zusammenhang womöglich verzerrt. Tatsächlich liegt der Korrelationskoeffizient für die EU15-Staaten allein sogar bei 0,52.

Eine hohe Spezialisierung auf die Industrie muss allerdings mit der internationalen Arbeitsteilung und den komparativen Vorteilen der Länder vereinbar sein. DeIndustrialisierung kann die Folge eines ganz normalen Strukturwandels sein, der sich aus überdurchschnittlichen Produktivitätssteigerungen in der Industrie und aus Verschiebungen der internationalen Spezialisierung ergibt. Dieser Strukturwandel ist ein Fortschritt, wenn die freigesetzten Arbeitskräfte im Dienstleistungssektor und in anderen Branchen neue, ertragreiche Beschäftigung finden und die Investitionen anderswo rentabler zum Einsatz kommen und mehr Einkommen erwirtschaften. Der Strukturwandel wird jedoch dann zum Problem, wenn es an Innovation fehlt und an anderer Stelle nichts Neues entsteht. In diesem Fall kann De-Industrialisierung dazu führen, dass ganze Regionen mit hoher Arbeitslosigkeit zu kämpfen haben, Industriearbeitsplätze durch schlecht bezahlte Jobs in wenig produktiven Dienstleistungen ersetzt werden und die Erwerbseinkommen vieler Haushalte sinken.

Die Nachfrage ist offensichtlich nicht der Grund für den Trend zur De-Industrialisierung (vgl. Rodrik, 2015). Peneder und Streicher (2016) zeigen für die EU von 1995 bis 2011, dass der Anteil der Güter des Verarbeitenden Gewerbes am Konsum kaum gefallen ist.11 Dagegen hat sich der Anteil des Verarbeitenden Gewerbes an der Produktion (nach der BWS) deutlich reduziert.12 Der Aufstieg Chinas und anderer Schwellenländer folgt einem starken Wachstum der Industrie, die auf den Weltmarkt drängt und zu Produktionsverlagerungen führt. In den USA fällt die De-Industrialisierung mit dem zunehmenden industriellen Output von China zusammen. Der Aufholprozess steigert auch in Europa und in Österreich die Exporte, führt aber auch zu mehr Importen von Industriegütern und löst einen Strukturwandel aus. Dazu kommt eine gewisse Verlagerung der Produktion innerhalb der EU in die NMS. Der Anteil der industriellen Produktion in der EU liegt aber nach wie vor höher als der Konsum. Offenbar konnte Österreich sich in dieser Neuordnung der internationalen Arbeitsteilung im Gegensatz zu anderen etablierten Industriestaaten bislang gut behaupten.

Die De-Industrialisierung ist damit zumindest teilweise ein künstliches Phänomen. Die Industriefirmen lagern laufend Teile der Wertschöpfung aus (Outsourcing), wenn andere Unternehmen besser und billiger anbieten, sparen damit Kosten und bleiben wettbewerbsfähig. Solche Entscheidungen spalten die industrielle Wertschöpfungskette global auf. Die standardisierte Produktion geht dabei häufig in kostengünstige Schwellenländer. In den Industrieländern konzentrieren sich die Unternehmen immer mehr auf die wissens- und technologieintensiven Teile der Wertschöpfung, die der Produktion vorund nachgelagert sind. Traditionelle Industrieunternehmen spezialisieren sich zunehmend auf Forschung, Entwicklung, Engineering, Design, Marketing und Vertrieb und mutieren dabei schleichend zu produktionsnahen Dienstleistern ohne Fabrik. Sie sind nach dieser

11 Zu Verbraucherpreisen fiel der Konsumanteil von 34,2\% auf 31,2\%. Zu Herstellungspreisen abzüglich Nahrung und Getränke schrumpfte der Rückgang auf einen Prozentpunkt zusammen, nämlich von 15,6\% auf 14,5\%.

12 Der Anteil an der BWS fiel von 20,1\% auf 15,8\%. Wegen unterschiedlicher Definitionen von „Industrie“ und „verarbeitendem Gewerbe" weichen die Zahlen in den Abbildungen teilweise von Peneder und Streicher (2016) ab (vgl. hierzu Kap. 2.1.3). 
Restrukturierung häufig F\&E-intensiver und weisen eine höher qualifizierte Beschäftigungsstruktur auf als vorher.

Obwohl die Unternehmen unverändert auf den gleichen Absatzmärkten konkurrieren, zählen sie nach ihrer Reorganisation häufig zum Dienstleistungssektor. Statistisch findet damit eine Verschiebung von der Industrie zum Dienstleistungssektor statt, obwohl die Unternehmen faktisch auf produktionsnahe Tätigkeiten spezialisiert bleiben. So ist offenbar Dänemark mehr als andere Innovation Leader von der „De-Industrialisierung“ betroffen. Bernard u.a. (2016) dokumentieren, dass ein erheblicher Teil der Firmen dabei nicht ausgeschieden ist, sondern weiterhin „nicht industriell produziert“ und sich zu produktionsnahen Dienstleistern entwickelt hat. Die Unterscheidung zwischen Industrie und Dienstleistungssektoren verwischt sich. Auch Automobilkonzerne verbinden ihre Produkte zunehmend mit Serviceangeboten (Degryse, 2016). Dazu zählt nicht nur die Entwicklung des selbstfahrenden Automobils, sondern auch die Ausstattung mit Software, die dem Benutzer zur Unterhaltung während der Fahrt und für andere Services zur Verfügung steht.

\subsubsection{Arbeit und Beschäftigung}

Die neuere Forschung (Acemoglu und Autor, 2011; Autor und Handel, 2013; Autor u.a., 2003) unterscheidet zwischen den Aufgaben (tasks) eines Jobs und den erforderlichen Fertigkeiten (skills) der ArbeitnehmerInnen Der Einsatz von Informations- und Kommunikationstechnologien (IKT) erleichtert vor allem die Auslagerung und Automatisierung von mittel bis hoch qualifizierten Aufgaben. Parallel zum Rückgang der Beschäftigung im industriellen Sektor senkt diese Entwicklung schon seit Jahrzehnten die Reallöhne insbesondere niedrig qualifizierter Männer (Acemoglu und Autor, 2011). Dieser Prozess leitete schließlich auch eine Polarisierung auf dem Arbeitsmarkt ein, indem die Arbeitsplätze sowohl für hoch qualifizierte und hoch bezahlte Arbeit als auch für niedrig qualifizierte Arbeit mit geringen Löhnen zunahmen.13

Eine Aufgabe ist umso schwieriger zu automatisieren, je mehr Interaktion und kognitive Fähigkeiten erforderlich sind. Anfällig für Automatisierung sind vor allem Jobs mit Aufgaben, die Informationsaustausch, Verkauf oder manuelle Tätigkeiten umfassen. Die OECD (Arntz u.a., 2016) berechnet die Veränderungen der Arbeitsnachfrage als Folge der Automatisierung und unterscheidet dabei zwischen Aufgaben und Berufen. Indem sie die Analyse auf die zu verrichtenden Aufgaben abstellt, kommt sie zum Ergebnis, dass die Automatisierung hauptsächlich einzelne Aufgaben und nicht so sehr ganze Berufe ersetzt. Die Automatisierung erfasst damit die verschiedenen Berufe viel gleichmäßiger als oft angenommen. Das mag die irreführende, aber weitverbreitete Vorstellung der weitreichenden Arbeitsplatzzerstörung entkräften. Allerdings wird die Automatisierung die Anforderungen und notwendigen Qualifikationen der betroffenen Berufe stark verändern und stellt die ArbeitnehmerInnen nichts desto weniger vor große Herausforderungen.

Tatsächlich zeigt die OECD-Studie, dass sich die Zahl der automatisierbaren Jobs von mitunter genannten $47 \%$ auf $9 \%$ reduziert. Die Unterschiede zwischen den Ländern sind auf Arbeitsplatzorganisation, bereits erfolgte Investitionen in die Automatisierung sowie Ausbildung der ArbeiterInnen zurückzuführen. Ebenso zeigt die Studie, dass der Anteil der

13 Langfristig geht die OECD jedoch davon aus, dass diese Polarisierung wieder abnimmt. 
gefährdeten Jobs ausgerechnet in Österreich mit 12\% am größten ist. Die heimischen ArbeiterInnen führen zwar typischerweise weniger automatisierbare Aufgaben durch als ihre KollegInnen in den USA; Österreich weist allerdings einen hohen Anteil niedrig und mittel qualifizierter ArbeiterInnen auf, die mehr automatisierbare Aufgaben verrichten. Die Größe der Industrie allein spielt offensichtlich nicht die entscheidende Rolle. Südkorea ist das Land mit dem höchsten Anteil der Industrie an der BWS, weist aber den niedrigsten Anteil gefährdeter Jobs auf. Dort führen die ArbeiterInnen eben vergleichsweise wenige automatisierbare Aufgaben durch und weisen zudem ein hohes Ausbildungsniveau auf.

Die Automatisierungswelle steigert die Produktivität und ersetzt repetitive, standardisierte Arbeit. „Industrie 4.0“ könnte bald die neue Realität sein, in der Roboter in den Fabrikhallen miteinander vernetzt sind und große Teile der traditionellen Arbeit verrichten. Profitieren werden hiervon vor allem SoftwareentwicklerInnen, IngenieurInnen, ForscherInnen und andere ArbeiterInnen mit den Fertigkeiten und der Ausbildung, die in dieser „schönen neuen industriellen Welt" erforderlich sind (Roubini, 2015). Führt technologischer Fortschritt zu Massenarbeitslosigkeit? Mit Sicherheit wandeln sich die nachgefragten Fertigkeiten. Die Arbeitslosigkeit kann temporär steigen, wenn die Qualifikationen sich nicht rasch genug an den Strukturwandel anpassen. Automatisierung ist jedoch ein allmählicher Prozess; d.h., es werden weniger die Jobs wegfallen, sondern die Aufgaben werden sich ändern; neue Arbeitsplätze und Berufe kommen an anderer Stelle hinzu; und in der Industrie freigesetzte Arbeit wird zudem verstärkt in den Dienstleistungssektor fließen. Bislang zeigen Studien eher das Gegenteil von massenhaften Jobverlusten (vgl. Valsamis, 2015), wobei ein zentrales Ergebnis ist: Die Anzahl gefährdeter Jobs ist umso geringer, je weiter ein Land in der Digitalisierung fortgeschritten ist.

\subsubsection{Industrie 4.0}

Automatisierung und Digitalisierung, also die Nutzung von computergestützten Maschinen und Robotern, das Internet der Dinge, Big Data und ähnliche neue Phänomene können die Arbeitswelt nachhaltig verändern. Dass mit dem technologischen Fortschritt die Arbeit ausgeht, kann wirtschaftshistorisch nicht bestätigt werden (vgl. z.B. Gregory u.a., 2016). Allerdings verändern sich Anforderungen und die Einsatzfelder der Arbeit. Die Zahl der Industrieroboter stieg zwischen 2007 und 2012 weltweit um 24\% und in den beiden folgenden Jahren um weitere 20\%. Zwischen 2014 und 2018 ist abermals mit einem Zuwachs von 57\% zu rechnen (Stettes, 2016). Neben Nordamerika und den asiatischen Ländern wie China, Südkorea und Japan erscheint Deutschland als ein Hauptanwender der Robotertechnik. Der Einsatz von Industrierobotern in Deutschland ist bislang allerdings eher begrenzt und konzentriert sich auf eine relativ kleine Zahl von Industrieunternehmen. Er ist von der Unternehmensgröße und vom Umfang der Losgrößen in der Produktion abhängig und vornehmlich bei mittlerer Produktkomplexität rentabel (Jäger u.a., 2015).

Graetz und Michaels (2015) zeigen für 17 Länder und verschiedene Industriezweige zwischen 1993 und 2007, dass die zunehmende Automatisierung sich positiv auf Arbeitsproduktivität und Wirtschaftswachstum auswirkt. Demnach erhöhte der Einsatz von Robotern das BIP-Wachstum um durchschnittlich 0,37\%, während die Gesamtzahl der Arbeitsstunden unverändert blieb. Auch nach der Arbeit von Kromann u.a. (2011) für sieben Länder auf Branchenebene steigt mit zunehmendem Einsatz von Industrierobotern die 
Arbeitsproduktivität. Die Autoren weisen zudem darauf hin, dass die Beschäftigung kurzfristig tendenziell sinken und erst längerfristig wieder steigen mag, wobei die Effekte nicht besonders signifikant sind. Kurzfristig, bei konstantem Output und Kapitalstock, dürfte der technische Fortschritt z.B. bei einer geringen Substitutionselastizität zwischen Arbeit und Kapital wohl mit Beschäftigungseinbußen einhergehen. Längerfristig führen die geringeren Grenzkosten zu mehr Investitionen, einem höheren Kapitalstock und steigern den Output.

Die Digitalisierung geht jedenfalls weit über die Industrie hinaus. So findet z.B. IW Köln (2015) in den unternehmensnahen Dienstleistungen einen höheren Digitalisierungsgrad als in der Industrie. Die Digitalisierung geht einher mit dem Anteil von digitalisierungsbezogenen an allen Patentanmeldungen in einer Branche und dem Umfang der aus hoch digitalisierten Branchen bezogenen Vorleistungen. Die höchsten Digitalisierungsgrade im Jahr 2012 weisen Sektoren wie Audiovisuelle Medien und Rundfunk (58,2 \%), Telekommunikation (52,4\%), IT- und Information (47,5\%), Finanzen $(47,3 \%)$ und Rechts-, Steuerberatung und Unternehmensberatung $(47,1 \%)$ auf (PROGNOS, 2015).

In digitalen Netzwerken wie Internet und Mobiltelefone werden riesige Mengen an persönlichen, kommerziellen, geographischen und verhaltensbezogenen Daten (Big Data) verfügbar (Degryse, 2016). Bislang konzentriert sich die Kapitalisierung der digitalen Ökonomie auf wenige Unternehmen wie Google, Facebook, Apple, Amazon und IBM. Sie sammeln riesige Datenmengen über ihre Kunden, wandeln diese mit speziellen Algorithmen in nutzbare Informationen um und machen sie auf speziellen Plattformen für mobile Anwendungen und weitere Geschäftsaktivitäten verfügbar. Der Zuwachs an Daten wächst exponentiell. So sind 90\% der heute im Internet verfügbaren Daten weniger als zwei Jahre alt. Der Big-Data-Sektor wächst um 40\% pro Jahr, das ist sieben Mal höher als das Wachstum des Informationsdatenmarkts (EC, 2015). Kennzeichnend für Big Data sind die 3 Vs: Volume, Velocity (Tempo der Datensammlung) und Variety. Die Kombination aus Volumen, Geschwindigkeit der Verfügbarkeit im virtuellen Raum und aus der Verschiedenartigkeit der Daten erfordert eine „intelligente“ Nutzung von Big Data mit speziellen Suchalgorithmen und Lernmaschinen (Degryse, 2016). Brynjolfsson und McAfee (2011) sprechen daher nicht nur von einer neuen digitalen Wirtschaft, sondern auch von einem zweiten Maschinenzeitalter, welches von der Expansion digitaler Daten und dem Robotereinsatz getragen wird.

Digitalisierung und Big Data ändern die Organisation der Unternehmen (Mettling, 2015). „Traditionelle Unternehmen“ passen die neuen Technologien für ihre Zwecke an und nutzen sie z.B. in Form von Teleworking, mobiles Arbeiten oder gemeinschaftliche Infrastrukturen. „Digitale Unternehmen“ verdanken die Gründung und ihre ganze Existenz den neuen Technologien. Die Umwälzungen im Unternehmenssektor werden starke Auswirkungen auf die Arbeit haben. Sie reichen von Arbeitsplatzschaffung (durch neue Sektoren, Produkte und Dienstleistungen), Arbeitsplatzveränderung (durch Digitalisierung, intelligente MenschMaschine-Interaktionen und neues Management) bis hin zum Arbeitsplatzabbau (durch Automatisierung und Roboterisierung) und Arbeitsplatzumschichtung (in Richtung digitale Plattformen, Crowd Sourcing und Sharing Economy). Roubini (2015) sieht diese Entwicklung pessimistisch. In den Fabrikhallen der Zukunft würden nur noch wenige hoch qualifizierte IngenieurInnen hunderte Maschinen betreuen, während andere nur noch niedrige Jobtätigkeiten wie z.B. Reinigungsarbeiten übernehmen können. Ford (2015) kommt zum Schluss, dass zunehmend auch Routineaufgaben hoch qualifizierter Arbeit wie 
Programmierer, Anwälte etc. automatisiert oder in Niedriglohnländer wie z.B. Indien ausgelagert werden können. Nach Brynjolfsson und McAfee (2011) besteht das größte Problem wohl darin, dass Organisation und Qualifikation der Arbeit den sich rasch wandelnden Anforderungen der Digitalisierung und Automatisierung hinterher hinken. Nach ihrer Ansicht müsse daher eine erfolgreiche Strategie die Arbeit in die Lage versetzen, mit den Maschinen vorne mitzuhalten anstatt gegen sie anzukämpfen.

Digitalisierung und Automatisierung werden auch Österreich umfassend betreffen. Arbeitsinhalte werden sich auch hier verstärkt von Routine- zu Nicht-Routine-Tätigkeiten verschieben (Peneder u.a., 2016). Berufe mit analytischen und interaktiven Tätigkeiten werden mehr Kompetenzen und höhere Qualifikationen erfordern. Zwar ist eine JobPolarisierung bislang kaum zu bemerken, doch wird der Druck auf manuelle RoutineTätigkeiten und auf gering Qualifizierte auch in Österreich weiter zunehmen. Die Verstärkung von IKT-Kompetenzen wird zum entscheidenden Faktor (Falck u.a., 2016). Die aktiv Beschäftigten haben die Erstausbildung abgeschlossen und müssen den raschen Wandel der Anforderungen daher durch massive Investitionen in Aus- und Weiterbildung bewältigen. Die Verstärkung von IKT-Kompetenzen in den Schulen bereitet nur die nächsten Generationen auf die neue Arbeitswelt vor. Dabei haben Internetkompetenzen auch eine positive Auswirkung auf spätere akademische Leistungen. Davon profitieren gerade die Studierenden mit einem niedrigeren sozialen Status (Pagani u.a., 2015). Tatsächlich kann eine Reform der Erstausbildung den Bestand der aktiv Beschäftigten nicht mehr erreichen. Sie wirkt sich viel zu langsam auf die Qualifikationen der gesamten Arbeitnehmerschaft aus. Umso wichtiger sind daher die Investitionen der Unternehmen in Aus- und Weiterbildung und das lebenslange Lernen der ArbeitnehmerInnen.

\subsection{F\&E und Unternehmenswachstum}

Innovative Wachstumsunternehmen schaffen Konsumentenwohlfahrt mit neuen Produkten und Anwendungen höherer Qualität zu günstigen Preisen. Mit ihren Produktivitätsgewinnen ermöglichen sie steigende Löhne und Gewinne und sind eine Quelle für Beschäftigung und neue Exportchancen. Dieser Abschnitt zeigt, wie F\&E-Investitionen die Wettbewerbsfähigkeit stärken und das Unternehmenswachstum anschieben. Bereits Schumpeter (1942) beschrieb den Prozess der „schöpferischen Zerstörung“, wonach Pionierunternehmen völlig neue Märkte erschließen, mit ihren Neuerungen existierende Märkte aufmischen und mit einem besseren Preis-Leistungsverhältnis die weniger leistungsfähigen Konkurrenten verdrängen. Etablierte Leitbetriebe können angesichts der Herausforderung durch neue Anbieter ihre Stellung nur behaupten, indem sie selbst mit permanenter Innovation ihre Produktpalette erneuern. Im Produktzyklus steuern neue profitable Angebote den Hauptteil der Gewinne bei, während reife Produkte zunehmend von der Konkurrenz attackiert werden und die Gewinnspannen dahinschmelzen. Wenn die Unternehmen sich nicht schnell genug anpassen und ihren Produktzyklus zu langsam erneuern, steigt das Risiko einer Übernahme durch erfolgreichere Konkurrenten oder einer Insolvenz. Was Schumpeter als kreative Zerstörung bezeichnet, bestätigen ManagerInnen regelmäßig in Befragungen, indem sie typischerweise Innovation als wichtigste Wachstumsstrategie nennen (vgl. z.B. PWC, 2013). 


\subsubsection{F\&E, Investition und Beschäftigung}

Der private Ertrag der F\&E kann nur in den später erzielbaren Umsätzen und Gewinnen bestehen. Damit die Unternehmen mit Innovationen Gewinne erzielen, müssen sie zuvor Investitionen tätigen, Produktionskapazitäten aufbauen und danach den Vertrieb organisieren. Dabei kann die Wertschöpfungskette in viele Schritte aufgespalten werden und innerhalb oder außerhalb des innovierenden Unternehmens stattfinden. Deshalb ist Innovation zwar zentral für die Unternehmensentwicklung, muss sich allerdings nicht automatisch in mehr Sachkapitalinvestitionen niederschlagen.

Abbildung 17: Bruttoinvestitionen und Bruttoinlandsausgaben für F\&E in \% des BIP, 1995-2015

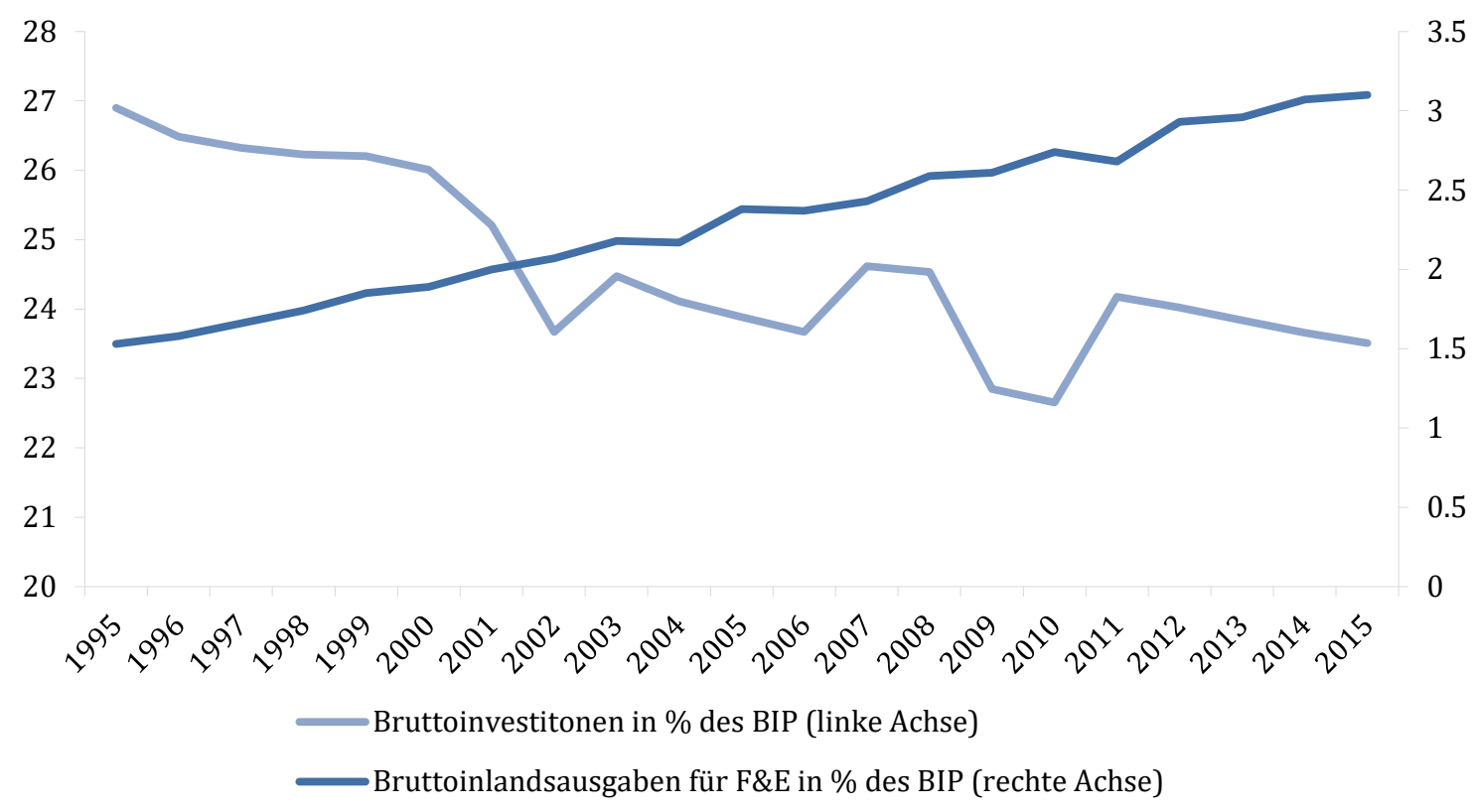

Quelle: Statistik Austria, eigene Berechnungen und Darstellung

Innovative Unternehmen können große Teile der Produktion an andere Firmen (Outsourcing) oder an Tochtergesellschaften (Direktinvestitionen) im In- oder Ausland auslagern und die bezogenen Vorleistungen und Komponenten zum Endprodukt „zusammenbauen“. Damit ist der Zusammenhang zwischen Innovation und Ausrüstungsinvestitionen innerhalb des Unternehmens aufgebrochen. F\&E schafft eine hohe Wertsteigerung, aber nicht unbedingt hohe Investitionen vor Ort. Auch in Österreich geht eine langfristig abnehmende Investitionsquote mit einer steigenden F\&E-Quote einher, wie Abbildung 17 zeigt.

Ein weiterer Grund für einen schwächeren Zusammenhang zwischen F\&E und Ausrüstungsinvestitionen ist, dass sich ein Teil der Unternehmen ganz auf F\&E, Engineering und Design spezialisiert. Sie treten gegen Lizenzgebühren die kommerzielle Verwertung der Technologien an andere Unternehmen ab. Solche Unternehmen sind überaus wissensintensiv und können äußerst profitabel sein, indem sie sich auf den wertschöpfungsintensivsten Teil der innovativen Produktion konzentrieren (Bernard u.a., 2016). Der Zusammenhang zwischen F\&E und Ausrüstungsinvestitionen schwächt sich ab, wenn Innovationen weniger kapitalintensiv werden. Die riesigen Unternehmenswerte in der neuen digitalen Ökonomie 
spiegeln vorwiegend Humankapital wider und sind wenig kapital- und beschäftigungsintensiv. Es dominieren Algorithmen und Internetplattformen anstatt Fabrikhallen.14

Hohe Lohnkosten und eine hohe Steuerbelastung erzeugen Druck, die Produktion in lohngünstige Länder auszulagern. Die österreichische Wirtschaft ist mit umfangreichen Direktinvestitionen sehr stark in Osteuropa vertreten. Neben den Lohnkosten und der Verfügbarkeit von qualifizierten Arbeitskräften bestimmen auch Transportkosten und die Nähe zum Absatzmarkt die Standortentscheidungen für die Investitionen. Damit Innovationen zu mehr Beschäftigung und Wertschöpfung im Inland führen können, braucht es Standortattraktivität für den Werkplatz Österreich, also für die der F\&E nachgelagerten Teile der Wertschöpfungskette. Damit innovative Unternehmen ihr Wachstumspotenzial voll ausschöpfen können, brauchen sie Zugang zum Weltmarkt und müssen ihre Produktions- und anderen Aktivitäten internationalisieren. Der Fokus auf Standortattraktivität darf keinesfalls die Internationalisierung der Unternehmen bremsen, sondern soll lediglich eine künstliche Produktionsverlagerung über das natürliche Niveau hinaus verhindern.

Der Zusammenhang zwischen F\&E und Produktion verändert sich im Laufe des Produktzyklus. In der ersten Phase besteht noch ein erheblicher Entwicklungs- und Perfektionierungsbedarf. Die Produktionsverfahren sind nicht standardisiert und müssen angepasst werden. Die Nähe zur F\&E erleichtert die Testläufe und hilft, Fehler („Kinderkrankheiten“) und Qualitätsdefizite gering zu halten. Umgekehrt können die Erfahrungen in der Produktion und im Vertrieb leichter an die F\&E-Abteilung zurückgemeldet werden, um das Angebot mit qualitätssteigernden Modifikationen zu perfektionieren. Mit fortschreitender Produktreife werden die Spezifikationen der Komponenten präziser und die Produktionsabläufe zunehmend standardisiert. Es wird leichter, die Fertigung von Komponenten an unabhängige und kostengünstigere Unternehmen auch im Ausland auszulagern. Die Verbindung von F\&E und Produktion wird loser. Umso eher können die Anlagen- und Ausrüstungsinvestitionen im Ausland erfolgen, wo die großen Absatzmärkte näher liegen und die Lohnkosten und andere schwer transportierbare Vorleistungen wie Energie billiger sind. Deshalb erfolgt die Produktionsverlagerung eher in späteren, reiferen Phasen des Produktzyklus (Antras, 2005). Tomiiura (2008) untersucht Mikrodaten über 118.300 japanische Industrieunternehmen und zeigt in der Tat, dass Outsourcing in F\&E-intensiven Branchen weniger bedeutend ist als in anderen. Innerhalb von Branchen gilt, dass Exporte vom Heimatstandort eine höhere F\&EIntensität aufweisen, als jene Produkte, die an unabhängige ausländische Unternehmen ausgelagert worden sind.

Ökonometrische Studien mit individuellen Unternehmensdaten aus wissensintensiven Branchen zeigen einen positiven Einfluss von F\&E-Investitionen auf Sachkapitalinvestitionen (Lach und Schankerman, 1989). Auch nach Chiao (2002) hängen Sachkapitalinvestitionen von erfolgreichen F\&E-Projekten ab. Die Kommerzialisierung von Innovationen verlangt nach neuen Investitionen zum Aufbau der Produktionskapazitäten. Die Arbeit weist zudem auf

14 Die drei größten Autoproduzenten aus Detroit erzielten im Jahr 1990 einen Umsatz von ca. 250 Mrd. US-Dollar, erreichten eine Marktkapitalisierung von 36 Mrd. und umfassten 1,2 Mio. MitarbeiterInnen. Die drei größten Unternehmen aus Silicon Valley erzielten 2014 gemeinsam einen Umsatz von 247 Mrd. Dollar, wiesen eine Marktkapitalisierung von über 1.000 Mrd. aus und erreichten dieses Ergebnis mit lediglich 137.000 Beschäftigten (The Economist, 17. September 2016). 
eine umgekehrte Kausalität hin. Hohe Sachkapitalinvestitionen in der Vorperiode stimulieren zusätzliche F\&E-Ausgaben in den Folgeperioden, um erfolgreiche Produkte weiterzuentwickeln und die Marktposition zu festigen oder weiter auszubauen.

Der Effekt von F\&E auf das Unternehmenswachstum ist keine rein mechanische Angelegenheit. Technologie alleine genügt nicht. Es gibt viele Entwicklungsfaktoren und Rahmenbedingungen, welche das Unternehmenswachstum positiv beeinflussen können, aber auch den Effekt von F\&E-Investitionen größer oder kleiner ausfallen lassen (siehe hierzu auch die Ausführungen zu Risikokapital und Strukturwandel in den Abschnitten 4.5 und 4.6). Dieselben F\&E-Investitionen der Unternehmen können mehr Wachstum und Beschäftigung anstoßen, wenn das Management professioneller, die Rechtssicherheit größer, der Zugang zum Kapitalmarkt leichter, der Marktzutritt weniger kostspielig, und die staatliche Vorleistung in Grundlagenforschung und Ausbildung von F\&E-Personal besser ist.

Je nach Kapitalintensität der Branche sind Investition und Beschäftigungszunahme aneinander gekoppelt. Die Bedeutung von F\&E für die Unternehmensentwicklung erschließt sich daher auch aus dem Beschäftigungswachstum. Abbildung 18 veranschaulicht den Zusammenhang anhand von Firmen, die in den FFG-Basisprogrammen erfasst sind, was dem größeren Teil der F\&E-aktiven Industrieunternehmen in Österreich entspricht. Das Diagramm zeigt einen positiven Zusammenhang zwischen F\&E-Intensität gemessen als F\&EAusgaben in Prozent des Umsatzes und dem Beschäftigungswachstum. Dieser Zusammenhang gilt sowohl für kleine als auch für große Unternehmen, wobei der Zusammenhang bei kleineren Unternehmen noch stärker ausgeprägt ist. Konkret ergibt sich etwa für Großunternehmen (mehr als 249 Beschäftigte) mit einer F\&E-Intensität von mehr als 20\% ein durchschnittliches jährliches Beschäftigungswachstum im Zeitraum 1996-2006 von 4\%. Im Gegensatz hierzu kam es bei Großunternehmen mit F\&E-Ausgaben unter 1,5\% des Umsatzes zu einem leichten Beschäftigungsrückgang.

Abbildung 18: Beschäftigungswachstum, F\&E-Intensität und Unternehmensgröße

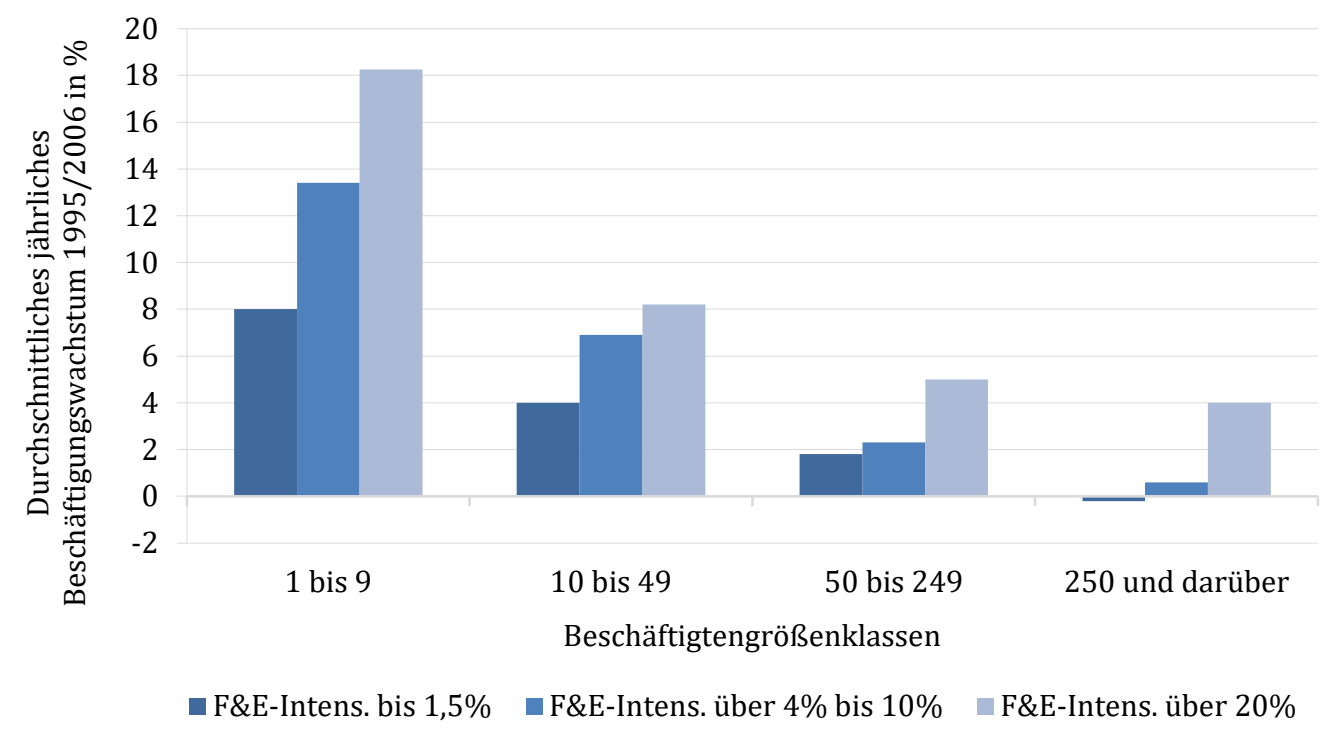

Quelle: Falk 2009, eigene Darstellung 
Die ökonometrische Analyse der FFG-Daten von 1996 bis 2006 zeigt ferner, dass eine Steigerung der F\&E-Personalintensität (F\&E-MitarbeiterInnen in Prozent der gesamten Beschäftigung) um 10\% zu einer Zunahme des Beschäftigungswachstums in den folgenden zwei Jahren von ca. 0,6 bis 1,8 Prozentpunkten führt. Dabei lag der Median der F\&EPersonalintensität im Jahr 2000 etwa in der Mitte des Untersuchungszeitraums bei 8,0. Der Median des jährlichen Beschäftigungswachstums der forschenden Unternehmen lag im selbigen Zeitraum bei ca. 3,5\%; im Lauf der Zeit ist der Effekt allerdings etwas kleiner geworden. Dass ein Großteil des Beschäftigungswachstums von einem sehr kleinen Teil innovativer und rasch wachsender Unternehmen stammt, wird in zahlreichen Studien bestätigt. Die meisten Firmen weisen kein Wachstum auf, während jeweils nur wenige schrumpfen oder wachsen (Coad u.a., 2014).

\subsubsection{Unternehmenswachstum im Branchen- und Ländervergleich}

Die Statistik Austria stuft Unternehmen mit einem Beschäftigungswachstum von mehr als $10 \%$ als rasch wachsend ein.15 Ihr Anteil beträgt in Österreich im Zeitraum 2008-2014 zwischen 6,5\% bis $9 \%$ und schwankt beträchtlich im Konjunkturverlauf. Während der Finanz- und Wirtschaftskrise konnten nur wenige Firmen ein Beschäftigungswachstum von mehr als 10\% realisieren. Daher erreichte ihr Anteil im Jahr 2010 mit 5,8\% ein Minimum und nahm erst im folgenden Aufschwung wieder zu. Tabelle 6 vergleicht die Anteile in Industrie und wissensintensiven Dienstleistungen. Die Unternehmen der Finanz- und Versicherungsbranche weisen die geringste Wachstumsdynamik auf. In der Informationsund Kommunikationsbranche sowie in den freiberuflichen/technischen Dienstleistungen sind die rasch wachsenden Unternehmen wesentlich häufiger vertreten. Die Industrie weist im Durchschnitt einen Anteil von 6,1\% auf und liegt damit um einen Prozentpunkt unter dem Wert der Gesamtwirtschaft. Auch für die Industrie gilt, dass der Anteil von 8,4\% im Vorkrisenjahr noch nicht wieder erreicht wurde.

Tabelle 6: Anteil rasch wachsender Unternehmen in Industrie und wissensintensiven Dienstleistungen

\begin{tabular}{llllllll}
\hline & $\mathbf{2 0 0 8}$ & $\mathbf{2 0 0 9}$ & $\mathbf{2 0 1 0}$ & $\mathbf{2 0 1 1}$ & $\mathbf{2 0 1 2}$ & $\mathbf{2 0 1 3}$ & $\mathbf{2 0 1 4}$ \\
\hline Insgesamt (ÖNACE 2008, B-S) & 9,1 & 6,5 & 5,8 & 6,4 & 6,9 & 7,8 & 7,5 \\
Information \& Kommunikation (IKT) & 13,8 & 10,2 & 8,9 & 10,0 & 9,8 & 13,0 & 12,5 \\
Freiberufliche/techn. Dienstleist. & 10,2 & 8,8 & 7,8 & 8,7 & 7,7 & 9,2 & 8,9 \\
Herstellung von Waren & 8,4 & 4,7 & 4,1 & 4,9 & 6,5 & 7,4 & 6,3 \\
Finanz- und Versicherungsleistungen & 7,2 & 4,2 & 3,7 & 2,6 & 3,4 & 4,8 & 5,2 \\
\hline
\end{tabular}

Bemerkung: Anteil schnell wachsender Unternehmen mit mind. zehn unselbständig Beschäftigten. Quelle: Statistik Austria, Statistik zu schnellwachsenden Unternehmen (ab 2015)

15 Die Statistik Austria stuft jene Unternehmen als rasch wachsend ein, die über einen dreijährigen Zeitraum ein durchschnittliches jährliches Beschäftigungswachstum von mehr als 10\% aufweisen. Um die Anteile der schnell wachsenden an allen Unternehmen nicht zu verzerren, werden alle Firmen ausgeschieden, die zu Beginn der Beobachtungsperiode weniger als 10 Beschäftigte aufweisen. Bei diesen würde nämlich schon ein kleiner absoluter Stellenzuwachs sehr hohe Prozentsteigerungen ergeben. 
In der Industrie herrschen höhere Kapitalintensitäten und Kostenvorteile durch Massenproduktion (Economies of Scale) vor. Die Unternehmen sind nur mit größeren Betriebsgrößen wettbewerbsfähig. Sie haben daher von vornherein mehr Beschäftigte und können umso weniger zusätzliches Beschäftigungswachstum erzielen. Der hohe Kapitaleinsatz erhöht die Eintrittsbarrieren, so dass die Großunternehmen dominieren und weniger junge innovative Wachstumsunternehmen zu erwarten sind. Im Gegensatz dazu kann die IKT-Branche aufgrund ihrer kleinteiligen Struktur eher Unternehmen mit einem Beschäftigungszuwachs von mehr als $10 \%$ hervorbringen.

Aus den rasch wachsenden Unternehmen rekrutiert sich die nächste Generation von multinationalen Konzernen. Innovation macht die Unternehmen groß und mit anhaltender Innovation und unter Ausnutzung von Kostenvorteilen durch Massenproduktion verteidigen sie den Vorsprung gegenüber der Konkurrenz und bleiben groß. Deshalb sind größere Unternehmen in der Regel forschungsintensiver, produktiver, internationaler und in Bezug auf ihren Standort mobiler. Mit einem kleinen Anteil rasch wachsender Unternehmen kann es schlussendlich auch nur wenige große Konzerne geben. Ist der Anteil größerer Unternehmen sehr gering, kann man auf potenzielle Wachstumsbarrieren und nicht realisierte Wachstumspotenziale schließen (Rubine u.a., 2012).

Tabelle 7: Unternehmensgrößen nach Beschäftigung im Industriesektor 2014, Anteile in \%

\begin{tabular}{llllll}
\hline & $\mathbf{0 - 9}$ & $\mathbf{1 0 - 1 9}$ & $\mathbf{2 0 - 4 0}$ & $\mathbf{5 0 - 2 4 9}$ & $\mathbf{2 5 0}$ \\
\hline Deutschland & 65,1 & 17,4 & 7,7 & 7,8 & 2,0 \\
Österreich & 72,8 & 11,1 & 8,5 & 5,7 & 1,8 \\
Schweiz & 53,9 & 20,8 & 14,4 & 9,1 & 1,8 \\
Dänemark & 71,2 & 12,0 & 9,2 & 6,4 & 1,3 \\
Verein. Königreich & 76,1 & 10,4 & 7,5 & 4,9 & 1,1 \\
Finnland & 81,0 & 8,1 & 6,1 & 3,9 & 0,9 \\
Belgien & 83,8 & 6,5 & 5,6 & 3,3 & 0,9 \\
Ungarn & 84,2 & 6,6 & 5,0 & 3,4 & 0,8 \\
Frankreich & 87,6 & 5,4 & 4,1 & 2,4 & 0,6 \\
Slowenien & 88,6 & 5,1 & 3,1 & 2,6 & 0,6 \\
Schweden & 88,1 & 5,1 & 3,8 & 2,4 & 0,6 \\
Niederlande & 86,5 & 5,5 & 4,3 & 3,1 & 0,5 \\
Tschechische Rep. & 92,9 & 2,6 & 2,3 & 1,8 & 0,5 \\
Slowakei & 92,9 & 3,4 & 1,8 & 1,5 & 0,4 \\
Italien & 82,9 & 9,9 & 4,8 & 2,1 & 0,3 \\
\hline
\end{tabular}

Quelle: Eurostat, Structural Business Statistics

Tabelle 7 zeigt die Größenverteilung der Industrieunternehmen nach Beschäftigten im Jahr 2014. Die Länder sind in absteigender Reihenfolge nach dem Anteil der Unternehmen mit mehr als 250 Beschäftigten sortiert. Österreich nimmt mit einem Anteil von 1,8\% gleichauf mit der Schweiz und hinter Deutschland den zweiten Platz ein, während Dänemark mit 1,3\% bereits deutlich weniger Großunternehmen beheimatet. Betrachtet man die nächst kleinere Größenklasse mit 50-249 Beschäftigten, so belegt Österreich mit 5,7\% den vierten Rang, während die Schweiz mit 9,1\% den Maximalwert erreicht. Österreich liegt damit deutlich vor 
Ländern wie den Niederlanden oder Belgien, aber auch wesentlich besser als die Nachbarländer in Ostmitteleuropa, die im Industriesektor als potenzielle Standortkonkurrenten gelten. Tabelle 7 verdeutlicht zudem die Strukturprobleme von Ländern wie Italien oder Frankreich, die gemessen an ihrem Entwicklungsniveau einen überaus kleinen Anteil großer Unternehmen aufweisen. In Summe ergibt der internationale Vergleich ein durchaus positives Bild vom Wachstum der heimischen Industrieunternehmen. Österreich ist eben nicht nur ein Land der KMU.

Das positive Bild von der Größenverteilung verdeckt allerdings eine unterdurchschnittliche Unternehmensdynamik in Österreich. Der Anteil rasch wachsender Unternehmen in der Industrie ist im internationalen Vergleich niedrig. Tabelle 8 zeigt, dass Österreich hier mit einem Durchschnittswert von 6,7\% am unteren Ende der Vergleichsländer steht. Ähnliche Länder wie z.B. Deutschland sind wesentlich dynamischer. Dies bedeutet, dass die „Aufstiegschancen“ zu gering sind und zu wenige der heimischen Unternehmen zu großen Konzernen mit vielen Beschäftigten heranwachsen.

Tabelle 8: Anteil rasch wachsender Unternehmen in der Industrie

\begin{tabular}{lllll}
\hline & $\mathbf{2 0 1 2}$ & $\mathbf{2 0 1 3}$ & $\mathbf{2 0 1 4}$ & Mittelwert \\
\hline Ungarn & 13,7 & 13,8 & 13,5 & 13,7 \\
Slowakei & 14,8 & 13,4 & 12,5 & 13,6 \\
Vereinigtes Königreich & 11,2 & 12,6 & 13,2 & 12,3 \\
Tschechische Republik & 12,6 & 11,5 & 8,7 & 10,9 \\
Schweden & 10,5 & 10,8 & 9,0 & 10,1 \\
Niederlande & 9,3 & 11,2 & 8,5 & 9,7 \\
Deutschland & 10,7 & 9,7 & 7,5 & 9,3 \\
Dänemark & 9,5 & 8,8 & 8,7 & 9,0 \\
Finnland & - & 10,5 & 7,2 & 8,8 \\
Slowenien & 8,4 & 8,0 & 7,2 & 7,8 \\
Frankreich & 7,6 & 6,9 & 6,8 & 7,1 \\
Österreich & 6,5 & 7,3 & 6,3 & 6,7 \\
Belgien & 6,7 & 6,2 & 5,9 & 6,3 \\
\hline
\end{tabular}

Quelle: Eurostat, Structural Business Statistics, bd_9pm_r2

Der European Innovation Scoreboard weist mit dem Beschäftigungsanteil rasch wachsender Unternehmen in innovativen Branchen für die Jahre ab 2012 eine neue Maßzahl aus. Der Indikator soll zeigen, inwieweit der Unternehmenssektor fähig ist, sich an neue Nachfragemuster anzupassen (European Commission, 2016). Der Anteil Österreichs liegt im Jahr 2015 mit einem Anteil von 19,4\% unter dem EU-Durchschnitt und konnte bislang noch keinen überdurchschnittlichen Wert erzielen (siehe Tabelle 9). Der Mittelwert von 17,3\% liegt gleichauf mit Finnland und vor Belgien sowie den Niederlanden. Allerdings verzeichnet Österreich mit einem jährlich steigenden Indikatorwert einen deutlich positiven Trend. Der Abstand zu den dynamischsten Ländern hat sich damit deutlich verringert. In Summe weisen die Statistiken allerdings auf ein Defizit an industrieller Dynamik bei innovativen Unternehmen hin. Dieses Ergebnis deckt sich auch mit Untersuchungen über weiter zurückliegende Zeiträume (Hölzl, 2011). 
Tabelle 9: Beschäftigungsanteile von Wachstumsunternehmen in innovativen Branchen, in \%

\begin{tabular}{lcccccc}
\hline & $\mathbf{2 0 1 2}$ & $\mathbf{2 0 1 3}$ & $\mathbf{2 0 1 4}$ & $\mathbf{2 0 1 5}$ & $\begin{array}{c}\text { Mittelwert } \\
\mathbf{2 0 1 2 - 2 0 1 5}\end{array}$ & Entwicklung \\
\hline Dänemark & 20,7 & 21,7 & 19,1 & 20,1 & 20,4 & - \\
Frankreich & 19,7 & 20,8 & 19,3 & 21,7 & 20,4 & + \\
Schweiz & 20,5 & 20,6 & 19,9 & 19,6 & 20,2 & - \\
Schweden & 21,0 & 19,2 & 18,9 & 19,6 & 19,7 & - \\
Deutschland & 18,5 & 18,5 & 19,5 & 21,0 & 19,4 & + \\
Slowakei & 19,4 & 16,9 & 19,3 & 20,9 & 19,1 & + \\
Ungarn & 18,2 & 18,7 & 19,1 & 19,2 & 18,8 & + \\
GB & 16,6 & 18,8 & 18,6 & 18,7 & 18,2 & + \\
EU 28 & 17,3 & 18,2 & 17,9 & 18,8 & 18,0 & + \\
Tschechien & 16,4 & 17,0 & 18,3 & 18,4 & 17,5 & + \\
Österreich & 16,0 & 16,7 & 17,2 & 19,4 & 17,3 & + \\
Finnland & 18,5 & 15,3 & 17,1 & 18,4 & 17,3 & - \\
Belgien & 17,4 & 17,3 & 15,4 & 16,9 & 16,8 & - \\
Niederlande & 17,1 & 16,4 & 16,2 & 16,9 & 16,6 & - \\
Slowenien & 15,2 & 15,3 & 15,3 & 16,0 & 15,4 & + \\
\hline
\end{tabular}

Anmerkung: Beschäftigung rasch wachsender Unternehmen in innovativen Branchen in \% der Beschäftigung aller Wachstumsunternehmen; Wert 2015 war größer (+) oder kleiner (-) als 2012 Quelle: European Innovation Scoreboard Data 2016

\subsubsection{Heterogene F\&E-Effekte auf das Unternehmenswachstum}

Die Forschung über den Einfluss von F\&E-Investitionen auf das Unternehmenswachstum arbeitet vorwiegend mit Mikrodaten (Literaturüberblicke bieten z.B. Audretsch u.a., 2014, und Coad, 2007). Die WissenschaftlerInnen operationalisieren Unternehmenswachstum mit verschiedenen Maßzahlen wie Umsatz, Beschäftigung, Unternehmenswerte und anderen Kennzahlen. Audretsch u.a. (2014, S. 745) fassen die Ergebnisse zusammen: Die empirische Evidenz zeigt einen positiven Effekt von Innovation auf das Firmenwachstum, der je nach Firmencharakteristiken, Marktverhältnissen und geographischer Umgebung unterschiedlich stark ausfällt. In der Folge zeigen einzelne Studien exemplarisch die wichtigsten Ergebnisse.

Produkt- und Prozessinnovation wirken unterschiedlich auf das Beschäftigungswachstum. Harrison u.a. (2008) zeigen für den Industriesektor in Deutschland, Frankreich, Spanien und Großbritannien, dass Produktinnovationen positive Beschäftigungseffekte auslösen, während Prozessinnovationen schwache Jobverluste zur Folge haben. Kosteneinsparungen durch Prozessinnovationen schlagen sich zwar in Jobverlusten nieder; sie schaffen jedoch auch den Spielraum für Preissenkungen, die den Absatz steigern und damit die Jobverluste teilweise wieder kompensieren. Auch die Ergebnisse von Hall und Oriani (2006) bestätigen den Konsens der empirischen Literatur im Hinblick auf positive Beschäftigungseffekte von Produktinnovationen und eines nicht eindeutigen Effekts von Prozessinnovationen.

Die empirische Forschung weist zudem darauf hin, dass vor allem rasch wachsende Unternehmen von F\&E-Investitionen profitieren, während ein „durchschnittliches“ Unternehmen mit F\&E weniger Umsatzwachstum erzielt (Coad, 2007). Diesen 
asymmetrischen Effekt von F\&E mit einer Konzentration des Beschäftigungswachstums bei rasch wachsenden Unternehmen bestätigten Capasso u.a. (2015) auch für die Niederlande. Ähnliches zeigt sich in Österreich (Falk, 2009): Bei den sehr schnell wachsenden Unternehmen im 80\%-Quantil (das sind die Top-20\% Unternehmen, während 80\% der anderen Unternehmen ein geringeres Beschäftigungswachstum verzeichnen) führt eine Zunahme der F\&E-Personalintensität um 10\% zu einer Steigerung des Beschäftigungswachstums in den folgenden zwei Jahren um ca. 1,8 Prozentpunkte. Dagegen liegt bei den $20 \%$ der am langsamsten wachsenden Unternehmen der entsprechende Effekt etwa bei Null.

Diese Ergebnisse sind mit einem typischen Lebenszyklus von Unternehmen gut vereinbar, wonach junge und F\&E-intensive Unternehmen in der ersten Lebensphase stark expandieren und den etablierten Anbietern Marktanteile abjagen. Große etablierte Firmen werden daher mit F\&E weniger ein weiteres Umsatzwachstum erzielen, sondern müssen ihre Produktpalette erneuern und qualitativ verbessern, um ihre Marktanteile zu behaupten. Zudem gilt, dass die Unternehmen ein umso größeres Wachstumspotenzial erschließen, je radikaler und grundlegender die Innovationen sind. Im Einklang damit finden Garcia-Manjon und Romero-Merino (2012) für 754 europäische Großunternehmen im Zeitraum 2003-2007, dass nur Hochtechnologieunternehmen mit ihren F\&E-Investitionen ein stärkeres Umsatzwachstum erzielen. Unternehmen im Niedrigtechnologiesegment und im Dienstleistungssektor mit geringer Wissensintensität sind dabei weniger erfolgreich. Demirel und Mazzucato (2012) untersuchen Unternehmensdaten der US-Pharmabranche von 1950 bis 2008, um den Effekt von F\&E auf das Umsatzwachstum festzumachen. Es zeigt sich, dass nur ein kleiner Teil der Unternehmen, nämlich junge und permanent innovierende, mit F\&E das Umsatzwachstum steigern können, nicht jedoch die großen. Als wichtigstes Ergebnis streichen die ForscherInnen heraus, dass es permanente Innovationen braucht, um mit F\&EInvestitionen nachhaltig expandieren zu können.

Die Unternehmen setzen mit ihren F\&E-Investitionen auf zukünftige Märkte und müssen daher antizipieren, wo der Bedarf der Nachfrager liegen wird. Dabei können die schnellsten Anbieter auf die größten Marktanteile hoffen. Mit Sicherheit werden für viele Jahrzehnte Umweltschutz und die Ökologisierung der Wirtschaft eine dominierende Rolle spielen. Ein früher Einstieg in umweltfreundliche Technologien könnte also den Unternehmen größere Marktanteile in stark expandierenden Märkten verschaffen.16 Colombelli u.a. (2015) untersuchen einen Datensatz von fast einer Million Industrieunternehmen aus Frankreich, Italien, Spanien, Schweden, Großbritannien und Deutschland für die Jahre 2002-2010, um die Effekte von ökologischen Innovationen festzumachen. Wieder zeigt sich wie vorhin, dass vorwiegend die jungen und rasch wachsenden Unternehmen mit ökologischen Innovationen ein höheres Umsatzwachstum erzielen und weniger die etablierten Anbieter.

Falk (2014) hat für den österreichischen Industriesektor die quantitativen Effekte von F\&EInvestitionen auf das Unternehmenswachstum untersucht. Die Einführung eines neuen Produkts bringt demnach ein um 1,7 Prozentpunkte höheres jährliches Beschäftigungswachstum als bei Nicht-Innovatoren, während der differentielle

16 Beim Umweltschutz ist die Rolle der Unternehmen als Anbieter und Nachfrager zu unterscheiden. Wenn die Firmen hohe Umweltstandards erfüllen müssen, werden sie zu Nachfragern von Umwelttechnologien und müssen höhere Kosten verdauen. Sich selbst mit F\&E als führender Anbieter von Umwelttechnologien zu etablieren, schafft dagegen neues Wachstumspotential. 
Wachstumsbeitrag von Prozessinnovationen bei 1,3 Prozentpunkten liegt. In dieser Untersuchung ergibt sich also auch für Prozessinnovationen ein deutlich positiver Beschäftigungseffekt, wenngleich dieser geringer ist als bei Produktinnovationen.

\subsubsection{Wachstumshindernisse}

In Österreich steht einer relativ vorteilhaften Größenverteilung der Industrieunternehmen eine geringe Dynamik bei rasch wachsenden Unternehmen gegenüber. Gibt es Hindernisse, welche jungen Unternehmen den Weg zum Wachstum verbauen? Abschnitt 2 hat erste Hinweise auf einige ungünstige Rahmenbedingungen gegeben, die sich im mittelmäßigen Abschneiden Österreichs im Doing-Business Report der Weltbank zeigen. Dazu kommt der Mangel an privatem Wagniskapital, das für die Finanzierung junger Technologieunternehmen eine wichtige Rolle spielen könnte. Rubine u.a. (2012) gehen über beschreibende Statistiken hinaus und schätzen ein strukturelles Modell, um in der Tradition von Melitz (2003) das Unternehmenswachstum zu erklären. Dabei stellen sie auf drei Wachstumsbremsen für Exportunternehmen ab, nämlich (i) Innovationskosten, (ii) Handelskosten und (iii) Regulierungskosten, operationalisiert durch Gewinn- und Lohnsteuern.

Tabelle 10: Wachstumsbarrieren und Unternehmenswachstum, in \%

\begin{tabular}{lccccccc}
\hline & Österreich & Frankreich & Deutschland & Ungarn & Italien & Spanien & GB \\
\hline Innovationskosten & 1,16 & 1,19 & 1,19 & 1,22 & 1,16 & 1,17 & 1,24 \\
Handelskosten & 1,24 & 0,62 & 0,42 & 0,21 & 0,69 & 0,68 & 0,49 \\
Gewinnsteuern & 0,2 & 0,11 & 0,28 & 0,21 & 0,34 & 0,01 & 0,37 \\
Lohnsteuer & 0,13 & 0,11 & 0,11 & 0,1 & 0,14 & 0,16 & 0,16 \\
\hline
\end{tabular}

Bemerkung: Effekte einer Reduktion von Wachstumsbarrieren um 1\%

Quelle: Rubine u.a. (2012)

Tabelle 10 zeigt für sieben europäische Länder, wie stark eine Reduktion der aufgelisteten Wachstumsbarrieren um 1\% das Wachstum von Exportfirmen steigern könnte. In Österreich würde demnach eine Reduktion der Innovationskosten um 1\%, z.B. mittels gezielter Subventionen oder F\&E-Steueranreize, das Wachstum der Exportfirmen um 1,16\% steigern. Die Gewinn- und Lohnsteuern spielen dagegen eine wesentlich geringere Rolle. Ein großer Teil der Innovationskosten (nach der F\&E-Erhebung im Jahr 2013 etwa 50\%) fällt als Lohnkosten für F\&E-Personal an, ist zu 100\% abzugsfähig und damit bereits von der Steuer vollständig entlastet. Eine Variation des Steuersatzes kann daher die Innovationskosten nur schwach beeinflussen. Eine Innovationsprämie oder eine steuerliche Abzugsfähigkeit über $100 \%$ würde dagegen die Innovationskosten direkt senken und das Unternehmenswachstum kräftig anstoßen.

Neben einer Senkung der Innovationskosten beflügelt vor allem ein reibungsloser Zugang zu den großen Absatzmärkten das Wachstum der Exportfirmen. Erst der Marktzugang macht den Umsatz möglich, ohne den eine Innovation sich niemals rechnen könnte. Österreich ist das einzige Land in Tabelle 10, für welches eine Reduktion der Handelskosten sogar noch wichtiger zu sein scheint als eine weitere Absenkung der Innovationskosten. Auf alle Fälle ist eine Reduktion der Handelskosten gerade für kleine Länder wie Österreich, die den hoch 
spezialisierten innovativen Unternehmen einen viel zu kleinen Heimmarkt bieten, viel wichtiger als für große Länder. Je spezialisierter ein Unternehmen, desto geringer ist die Bedeutung des Heimmarktes und desto wichtiger der Zugang zum Weltmarkt. Die vielen Hidden Champions in Österreich exportieren zwischen 80 bis 100\% ihrer Produktion. Genau deshalb sind die EU-Mitgliedschaft und die Teilnahme an weltweiter Handelsliberalisierung für die Innovation im kleinen exportorientierten Österreich auch so entscheidend (Keuschnigg und Sardadvar, 2016).

Der erleichterte Handel hat noch weitere wachstumsfördernde Wirkungen. Wenn die durchschnittliche Produktivität steigen soll, muss das Gewicht der hoch produktiven Firmen zunehmen und jenes der weniger produktiven abnehmen. Geringe Handelskosten stimulieren den Handel und begünstigen die Expansion der hoch produktiven Exportfirmen, während die verschärfte Importkonkurrenz die weniger produktiven Firmen bremst. Neben diesem Produktivitätseffekt ist der Handel auch ein Kanal, auf dem sich das technologische Wissen leichter über die Grenzen ausbreitet. Der bessere Zugang zum weltweiten Wissensbestand erleichtert den Unternehmen die weitere Entwicklung und stärkt ihre Innovationsfähigkeit.17 Angesichts der geographischen Lage mitten in Europa und der Kleinheit des nationalen Marktes ist der Zugang zum großen europäischen Binnenmarkt für den Innovationsstandort Österreich von größter Bedeutung.

\subsubsection{Fördert Innovation die Krisenrobustheit?}

Mit F\&E stärken die Unternehmen ihre Wettbewerbsfähigkeit. Daher zeigt die empirische Evidenz, dass eine hohe F\&E-Intensität nicht nur das Unternehmenswachstum steigert, sondern auch die Krisenrobustheit stärkt. F\&E-aktive Unternehmen haben ein geringeres Risiko zu schrumpfen oder ganz aus dem Markt auszuscheiden. Wettbewerbsfähige Firmen können Nachfrageeinbrüche leichter auffangen und wirken daher stabilisierend. In einer Wirtschaft mit vielen F\&E-intensiven Unternehmen fallen Rezessionen tendenziell weniger drastisch aus. In der EU hat der Nachfrageeinbruch im Jahr 2009 unabhängig von ihrer Technologieintensität alle Unternehmen getroffen. Allerdings sind die Unternehmen der Spitzentechnologie wesentlich besser durch die Krise gekommen. Die anderen Industrieunternehmen mussten dagegen mit ca. $-10 \%$ einen drastischen Einbruch hinnehmen, und zwar ähnlich stark in allen drei nachfolgenden Technologieklassen. 18 In der darauffolgenden Erholung sind erneut die Unternehmen der Spitzentechnologie am raschesten und deutlichsten wieder auf einen Wachstumskurs eingeschwenkt und haben als einzige Gruppe das Vorkrisenniveau bereits wieder überschritten. Die Firmen der mittleren Hochtechnologie konnten am ehesten noch die Krise hinter sich lassen, während die weniger innovativen Unternehmen mit mittelniedriger und niedriger Technologie sich kaum mehr erholen konnten.

Im Unterschied zur gesamten EU mussten österreichische Industrieunternehmen deutlich geringere Produktionseinbrüche in Kauf nehmen. Auch in Österreich haben die

17 Die von Romer $(1986,1990)$ geprägte endogene Wachstumstheorie und eine breite, darauf aufbauende empirische Forschung betonen die Bedeutung solcher Wissens-Spillovers für die Innovation. Genau aus diesem Grund sind die sozialen Erträge der privaten Innovation viel höher als die privaten.

18 ec.europa.eu/eurostat/statistics-explained/index.php/Glossary:High-tech_classification_of_manufacturing_industries/de enthält die genaue Definition zu den Technologieklassen. 
Spitzentechnologieunternehmen die Krise am besten gemeistert und sich im Anschluss am dynamischsten von allen entwickelt (Abbildung 19). Die Unternehmen mit mittlerer Hochtechnologie haben sich gut erholt und ebenfalls das Vorkrisenniveau bereits wieder überschritten. Eher erstaunlich ist, dass in Österreich die Unternehmen der niedrigsten Technologieklasse sich als krisenrobuster erwiesen haben als die Firmen in der nächst besseren, mittelniedrigen Technologieklasse. Nichtsdestoweniger gilt auch für Österreich, dass die technologieintensiveren Firmen sich durch größere Krisenrobustheit auszeichnen als die weniger innovativen Unternehmen. Dieses Bild zeigt sich nicht nur bei der Industrieproduktion, sondern auch bei der Entwicklung des Beschäftigungsniveaus von Unternehmen unterschiedlicher Technologieklassen. Innovative Unternehmen müssen in einer Krise weniger Beschäftigte abbauen als weniger innovative.

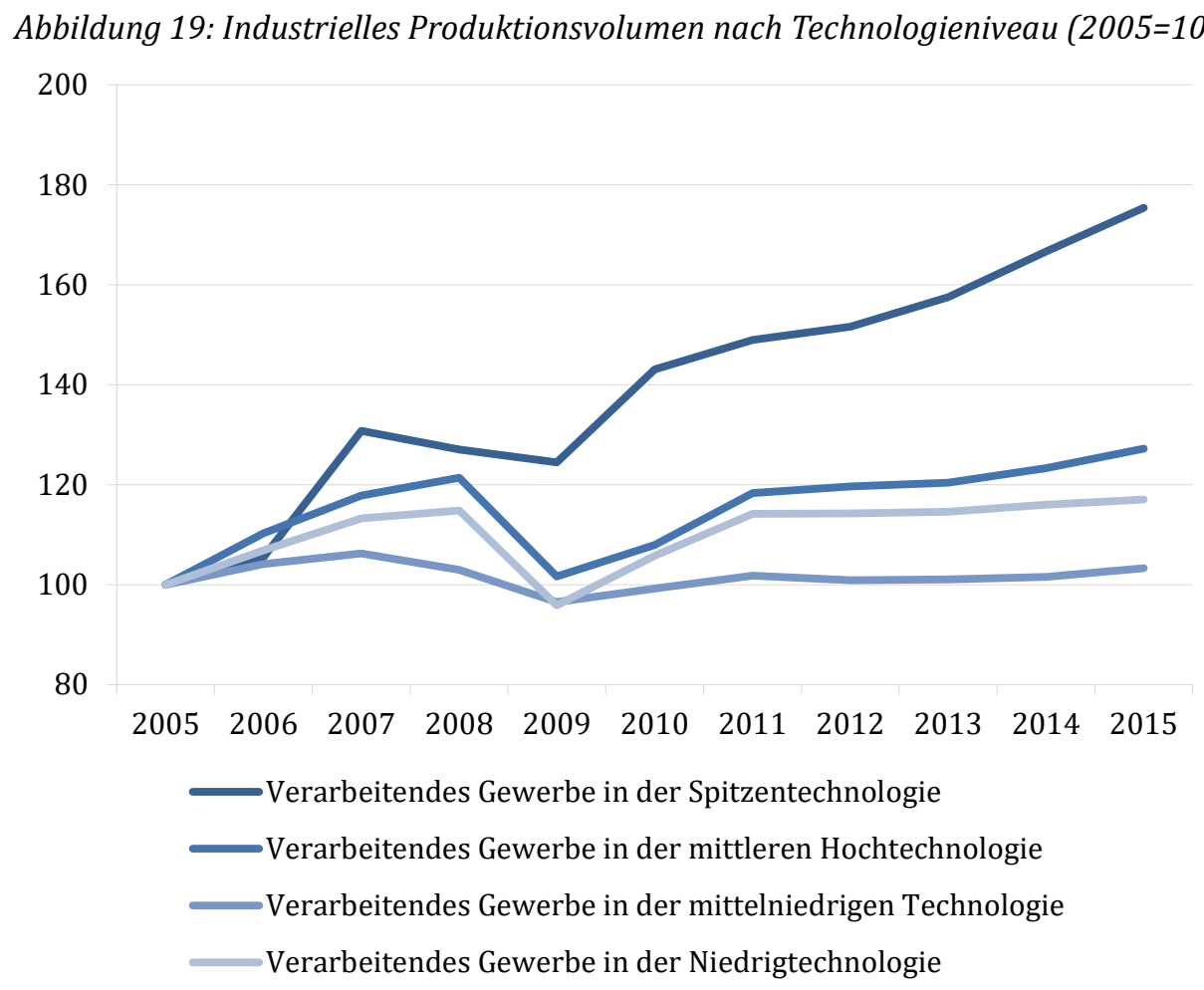

Quelle: Eurostat, Konjunkturstatistik

Auch Campagna u.a. (2012) bescheinigen der österreichischen Industrie eine hohe Krisenrobustheit. Die ForscherInnen werten eine umfassende Befragung von Industrieunternehmen in Österreich, Frankreich, Deutschland, Ungarn, Italien, Spanien und Großbritannien 19 aus und kommen zum Ergebnis, dass die heimische Industrie die Wirtschaftskrise von 2008/2009 am besten gemeistert hat. Die Ursache sehen sie vor allem im starken Fokus der österreichischen Industrieunternehmen auf Investition und Innovation. Die für die heimische F\&E so wichtigen ausländischen Tochtergesellschaften haben dabei im Vergleich zu den heimischen Unternehmen zwar stärker bei Beschäftigung und

19 Siehe http://bruegel.org/publications/datasets/efige/. 
Sachkapitalinvestitionen gekürzt, dafür aber weniger bei F\&E-Investitionen eingespart. Tatsächlich zeigen Untersuchungen der OECD (2015), dass F\&E-Investitionen generell eine geringere Volatilität als Sachkapitalinvestitionen aufweisen und in der großen Rezession deutlich weniger eingebrochen sind als Investitionen in Maschinen und Ausrüstung.

Peters u.a. (2014) untersuchen Daten aus fünf europäischen Innovationserhebungen, um die konjunkturellen Beschäftigungsschwankungen von Industrieunternehmen in Abhängigkeit von ihrer Innovationsstrategie zu erklären. Abbildung 20 zeigt das Beschäftigungswachstum im Aufschwung, Boom, Abschwung und in der Rezession in Abhängigkeit vom Innovationstyp. Innovative Unternehmen steigern die Beschäftigung im Boom wesentlich stärker und schränken sie in der Rezession weniger stark ein als nicht innovative Unternehmen. Der Beschäftigungsverlust in der Rezession ist nur halb so groß $(-2,5 \%$ anstatt $-5,6 \%)$. Ein ähnliches Muster zeigt sich in der Unterscheidung zwischen Produkt- und Prozessinnovationen. Industrieunternehmen mit Produktinnovationen büßen in der Rezession mit -2,2\% weniger Beschäftigung ein als Firmen mit Prozessinnovatoren $(-3,2 \%)$.

Abbildung 20: Innovation und Beschäftigungswachstum im Konjunkturzyklus, Industrie 1998-2008, in \%

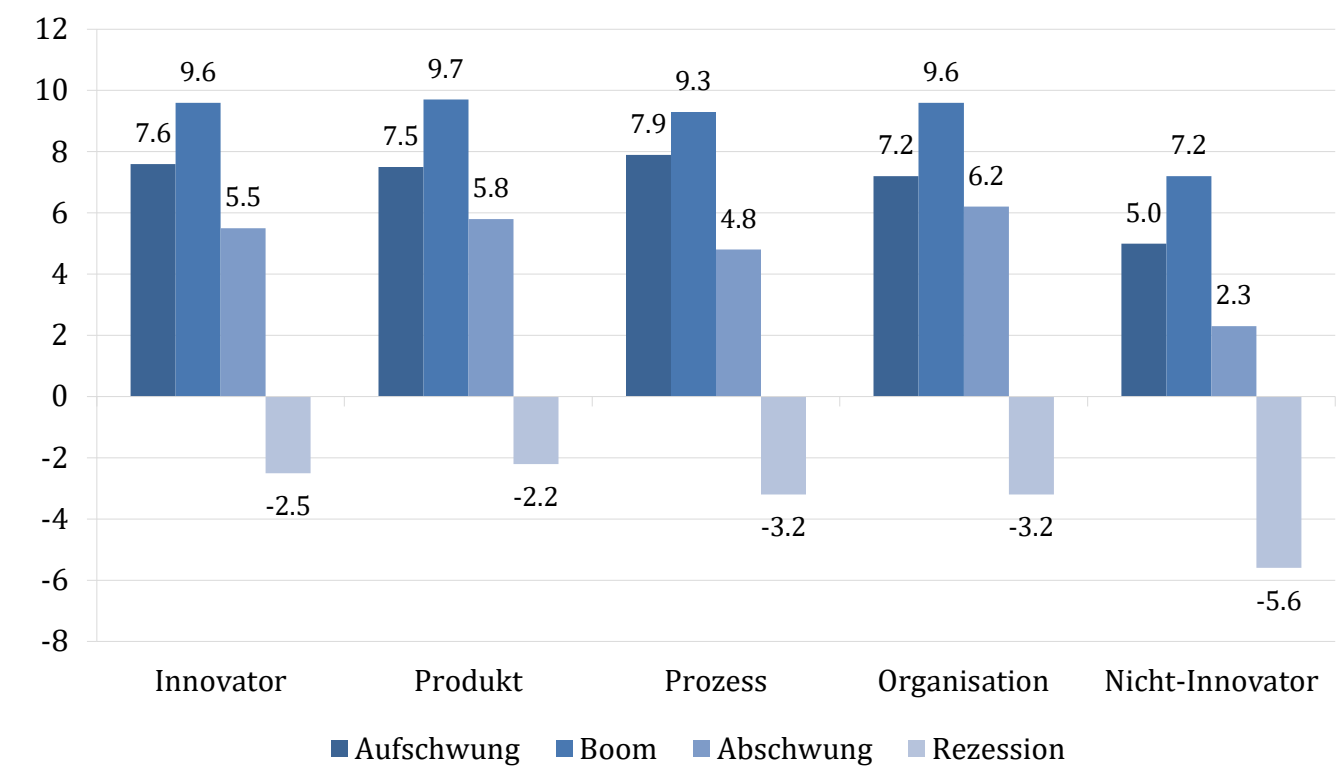

Quelle: Peters u.a. (2014)

Mit ökonometrischen Methoden gelingt es, das Beschäftigungswachstum von Unternehmen wie in Abbildung 21 in einzelne Elemente innerhalb jeder Konjunkturphase zu zerlegen, um den separaten Beitrag von Produkt- und Prozessinnovationen sowie von Veränderungen der Nachfrage nach alten und neuen Produkten zum Konjunkturzyklus deutlich zu machen. Neue Produkte haben einen negativen Verdrängungseffekt auf alte Produkte, aber gleichzeitig einen positiven Beschäftigungseffekt durch die Erschließung neuer Absatzmärkte. Die beiden letzten, schraffierten Balken zeigen diese beiden gegenläufigen Effekte. Der drittletzte Balken illustriert den Nettoeffekt. Dieser zusammen mit den vier Balken davor ergibt die gesamte Beschäftigungsänderung im ersten roten Balken. 
Abbildung 21: Innovationen und Beschäftigungswachstum im Konjunkturzyklus, 1998-2008, in \%

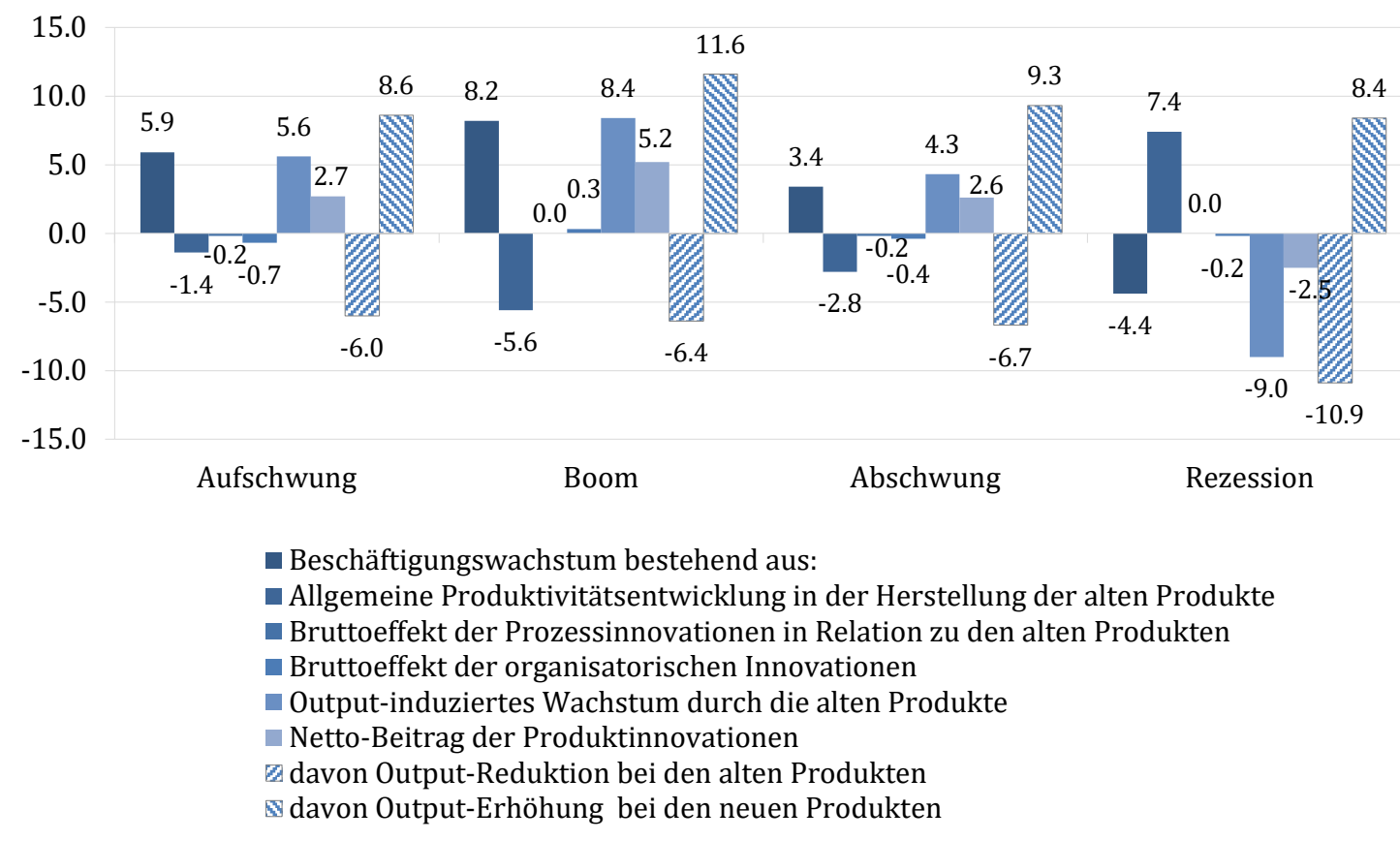

Quelle: Peters u.a. (2014)

Abbildung 21 zeigt für alle Konjunkturphasen außer für die Rezession ein positives Beschäftigungswachstum. Die Produktivitätsentwicklung (zweiter Balken) wirkt antizyklisch, indem sie den Beschäftigungszuwachs im Aufschwung, Boom und Abschwung bremst und gleichzeitig mit einem positiven Beschäftigungsbeitrag den Beschäftigungsverlust in der Rezession dämpft. Die nächsten zwei Balken in Abbildung 21 geben die Effekte von kostensparenden Prozess- und Organisationsinnovationen wieder, die in der Regel über alle Konjunkturphasen leicht negativ auf die Beschäftigung wirken. Die Balken 5 und 6 zeigen die Wirkungen von alten und neuen Produkten. Im Konjunkturzyklus verändern sich die Nachfrage nach beiden Produkttypen und in der Folge die Beschäftigungsbeiträge jeweils in dieselbe Richtung. In einer Rezession tragen beide negativ, ansonsten positiv zum Beschäftigungswachstum bei. Allerdings ändert sich das Verhältnis der Wachstumsbeiträge im Konjunkturzyklus markant. Im Aufschwung, Boom und Abschwung trägt die Nachfrage nach alten Produkten wesentlich stärker zum Beschäftigungszuwachs bei. In der Rezession bricht dagegen die Nachfrage nach alten Produkten wesentlich stärker ein und verursacht einen drastischeren Beschäftigungsverlust, der dreimal so stark ist wie bei neuen Produkten (-9\% anstatt -2,5\%). Alte Produkte haben häufig nahe Substitute und sind einem stärkeren Preiswettbewerb ausgesetzt, sodass die Nachfrage wesentlich stärker schwankt. Dagegen sind Produktinnovationen neu und haben noch wenig Konkurrenz. Der Preiswettbewerb ist weniger hart. Die Konsumenten können nur schwer auf andere Substitute ausweichen, sodass sich die Nachfrage stabiler entwickelt. Innovative Unternehmen mit einem hohen Anteil neuer Produkte in ihrem Angebotsportfolio 
sind krisenresistenter. Viele Unternehmen mit innovativen Produkten stabilisieren somit das Beschäftigungswachstum im Konjunkturverlauf und federn Krisen besser ab.

\subsection{F\&E und Internationalisierung der Unternehmen}

Mit radikalen Produktinnovationen starten die Unternehmen den Produktzyklus und erschließen ganz neue Märkte. Mit qualitätssteigernden Innovationen bauen sie ihre Marktanteile aus und schützen den Absatz vor der Konkurrenz. Mit Prozessinnovationen sparen sie Kosten und halten sich preislich wettbewerbsfähig. Im Zuge des Unternehmenswachstums erweitern sie die Produktpalette, nehmen obsolete und unprofitabel gewordene Angebote vom Markt und ersetzen diese durch ein neues Angebot. In den großen Konzernen treibt permanente Innovation den Produktzyklus und ersetzt diese alte durch neue Angebote. Bei den vielen kleinen Unternehmen erfolgt die Erneuerung der Produkte und Dienstleistungen im Wettbewerb durch Marktzutritt und -austritt. Neugründungen machen zu jedem Zeitpunkt etwa 7\% der Unternehmen aus, und etwa ebenso viele scheiden z.B. durch Übernahmen oder Insolvenzen aus.

Unternehmen starten klein und wachsen mit anhaltender Innovation. Wenn der Erfolg der Innovation ausbleibt, schrumpft die Profitabilität und sie müssen konsolidieren, sich gesund schrumpfen oder ganz ausscheiden. In diesem Lebenszyklus der Unternehmen ist ein typisches Muster der Internationalisierung angelegt. Unternehmen starten klein und national. Je spezialisierter das Angebot, desto weniger reicht die potenzielle Nachfrage auf dem heimischen Binnenmarkt und desto mehr müssen sie den Weltmarkt erschließen, indem sie das Exportgeschäft aufnehmen und schließlich mit Direktinvestitionen ausländische Produktionskapazitäten aufbauen. Mit Direktinvestitionen bedienen sie den Markt vor Ort und nahe am Konsumenten, um Transportkosten zu sparen. Mit Direktinvestitionen oder Outsourcing an unabhängige Produzenten verlagern sie Teile der Produktion von wichtigen Komponenten und Vorleistungen, um die Produktionskosten zu senken. Je spezialisierter die Nischenanbieter sind und je kleiner der heimische Binnenmarkt ist, desto eher müssen die Unternehmen den Export aufnehmen und Direktinvestitionen tätigen, und desto internationaler ist die Wirtschaft im Querschnitt der Unternehmen ausgerichtet.

\subsubsection{F\&E, Produktivität und Exporte}

Im Zuge des Wachstums innovativer Unternehmen wird vor allem in kleinen Ländern der Binnenmarkt rasch zu klein. Die Unternehmen müssen ausländische Märkte erschließen. Der Markteintritt im Ausland ist jedoch mit hohen Fixkosten verbunden und stellt eine große Investition dar, die sie erst mit dem zusätzlichen Absatz auf dem Weltmarkt erwirtschaften müssen. Zu diesen Fixkosten des Exportgeschäfts zählen Marktforschung, Anpassung der Produktspezifikationen nach abweichenden ausländischen Vorschriften und Standards, Produktzulassung, Aufbau eines Vertriebsnetzes, Verhandlungen und Vertragsgestaltung mit Großhändlern und vieles mehr. Dazu kommen die laufenden Kosten für Zollformalitäten im Handel außerhalb der EU und Transportkosten je nach Produkttyp und Entfernung. Beim Aufbau von ausländischen Produktionskapazitäten kommen weitere Fixkosten wie z.B. für Management, Unternehmenskontrolle und Administration zur Erfüllung ausländischer Steuer-, Arbeitsmarkt- und Regulierungsvorschriften hinzu. Diese zusätzlichen Fixkosten müssen die Firmen durch höhere Produktivität und Profitabilität kompensieren. Zudem 
setzen sich international ausgerichtete Firmen einem wesentlich schärferen Wettbewerb aus, weil sie nicht nur auf die inländische, sondern auch auf die weltweite Konkurrenz treffen. Aus diesen Gründen müssen international orientierte Firmen wesentlich produktiver und größer sein als heimische Firmen. Nach Ergebnissen von Helpman u.a. (2004) für die USA sind Exportfirmen um 39\% produktiver als rein national orientierte Firmen, und multinationale Unternehmen um 54\%, also um 15 Prozentpunkte produktiver als Exportfirmen.

Die höhere Produktivität erlangen die Unternehmen (i) durch permanente Innovation und (ii) durch Ausnutzung von Größenvorteilen (Skalenerträge). Vor allem die Präsenz von Fixkosten führt zu abnehmenden Stückkosten und damit zu einer höheren preislichen Wettbewerbsfähigkeit durch Massenproduktion. Aus diesen Gründen sind Exportfirmen innovativer und größer als rein nationale Firmen. Zu den Skalenerträgen innerhalb eines Unternehmens kommen externe Skaleneffekte, die $\mathrm{zu}$ geringeren Stückkosten der Unternehmen bei zunehmender Größe der gesamten Branche führen. Die Produzenten sind dabei auf verschiedenste Inputs, wie hochspezialisierte Dienstleistungen, Zwischenprodukte und Arbeitskräfte, angewiesen. Je größer die Branche ist, desto größer ist das Netzwerk der Zulieferer und desto größer die Zahl der spezialisierten ArbeitnehmerInnen. Desto größer ist zugleich auch die Chance, die kostengünstigsten Inputs in der geeigneten Qualität zu erhalten. Deshalb gibt es nicht nur eine kritische Unternehmensgröße, sondern auch eine kritische Größe für die gesamte Branche, welche $\mathrm{zu}$ weiteren Kostenvorteilen führt und den Unternehmen Wettbewerbsvorteile verschafft.20

Das Produktivitätsniveau schwankt sehr stark zwischen verschiedenen Unternehmen nicht nur in der Gesamtwirtschaft, sondern auch innerhalb einer Branche (Bartelsman u.a., 2013), wobei die produktiveren Unternehmen typischerweise international orientiert sind. Bernard u.a. (2012) zeigen für die USA, dass sich Exporteure in mehreren Eigenschaften systematisch von nicht exportierenden Firmen unterscheiden:

- Innovation: Exportfirmen investieren mehr in F\&E und sind humankapitalintensiver.

- Finanzierung: Exportfirmen finanzieren sich angesichts des höheren Risikos mehr mit Eigenkapital, allerdings in Europa weniger als in den USA.

- Humankapital: Löhne und Gehälter stellen mehr auf Leistung ab und das Management ist tendenziell professioneller.

- Eigentümerstrukturen: Die Präsenz ausländischer Investoren und die Integration in globale Wertschöpfungsketten wirken sich positiv auf die Exportneigung aus.

Altomonte u.a. (2012) betonen, dass kleine Unternehmen, wenn sie mit ihren Innovationen erfolgreich sind, produktiver werden, stark wachsen und bald das Exportgeschäft aufnehmen müssen, um ihr Potenzial auszuschöpfen. Die ForscherInnen untersuchen einen umfangreichen Datensatz von knapp 15.000 Firmen aus sieben EU-Mitgliedstaaten, darunter 443 Firmen aus Österreich, und kommen zu folgenden Ergebnissen:

- Je höher die Produktivität ist, umso eher sind die Firmen Exporteure. Im obersten Dezil der produktivsten Firmen sind mehr als 80\% im Export tätig.

20 Das vielleicht bekannteste Beispiel für solche Netzwerkvorteile ist das Silicon Valley in Kalifornien, wo sich eine kritische Größe an Pools von Anbietern und Arbeitskräften der Hard- und Softwareindustrie entwickelt hat, die den Start-ups lokale Kostenvorteile beschert. 
- Unter sonst gleichen Umständen (d.h., es wird für andere Faktoren kontrolliert) ist die Korrelation zwischen Export und Produktivität umso höher, je größer die Firmen sind.

F\&E-Investitionen haben einen zentralen Einfluss auf die Produktivität und Exportneigung der Unternehmen. Dieser Zusammenhang ist empirisch durch viele Studien belegt; so zeigen Esteve-Pérez und Rodríguez (2013) in einer ökonometrischen Studie für Spanien unter Berücksichtigung weiterer Einflussfaktoren eine um 22,5\% höhere Wahrscheinlichkeit, dass KMU, die in der Vorperiode in F\&E investiert haben, ihre Güter exportieren werden. Für Belgien zeigen Muûls und Pisu (2009), dass Handel treibende Unternehmen produktiver sind als solche, die nur den Heimmarkt bedienen.

Innovation treibt die Internationalisierung der Unternehmen, und umgekehrt ist der internationale Wettbewerb ein Produktivitäts- und Innovationsansporn für heimische Firmen. Mion und Zhu (2013) zeigen für Belgien, dass die Importkonkurrenz aus China zwar das Beschäftigungswachstum der Firmen bremst, aber den Anteil höher qualifizierter Beschäftigung im Verarbeitenden Gewerbe mit niedrigem Technologiegrad kräftig steigert (SkillUpgrading). Von der beobachteten Verbesserung der Qualifikationsstruktur gehen 27\% auf die Personalpolitik innerhalb der Firmen zurück, und 48\% auf die Expansion von Firmen mit höher qualifizierter Beschäftigung auf Kosten jener mit weniger qualifizierter Belegschaft. Iacovone u.a. (2013) zeigen, wie der Importwettbewerb durch chinesische Anbieter die mexikanischen Produzenten zu Produktivitätssteigerungen zwingt. Die Firmen geben weniger profitable Produkte auf oder scheiden ganz aus, während die produktivsten Firmen weiter expandieren. In der Folge steigt die durchschnittliche Produktivität und ermöglicht höhere Einkommen. Die Ergebnisse von Aghion u.a. (2009) und Aghion (2016) betonen dieselben heterogenen Effekte des internationalen Wettbewerbs auf unterschiedliche Firmen, wonach die Ansiedlung ausländischer Betriebe grundsätzlich das Potenzial hat, die Technologiegrenze im Gastland nach außen zu verschieben. Allerdings sind zwei Effekte zu unterscheiden:

- Das Produktivitätswachstum von Firmen, die nahe der globalen Technologiegrenze operieren, beschleunigt sich. Sie erhalten ihre Konkurrenzfähigkeit und verteidigen ihre Marktstellung, indem sie verstärkt in F\&E investieren.

- Dagegen verlangsamt sich das Produktivitätswachstum von Firmen, die einen technologischen Rückstand haben. Die Importkonkurrenz führt zu Resignation bis hin zur Geschäftsaufgabe.

Ökonometrische Schätzungen (Aghion, 2009) zeigen, dass die Erhöhung des Anteils ausländischer Betriebe um einen halben Prozentpunkt für jene einheimischen Betriebe, die sich hinsichtlich ihrer Entfernung zur Technologiegrenze im obersten Dezil befinden, die Arbeitsproduktivität um zwei Prozentpunkte erhöht. Demgegenüber liegt der Effekt für einen einheimischen Betrieb, der technologisch den Median repräsentiert, bei 0,7 Prozentpunkten. Einheimische Betriebe, die sich hinsichtlich ihrer Entfernung zur Technologiegrenze im untersten Dezil befinden, erleiden einen Produktivitätsrückgang von 0,6 Prozentpunkten.

Darüber hinaus kann Internationalisierung an sich die Innovationstätigkeit der Unternehmen anregen (Aw u.a., 2011). In diesem Zusammenhang heben Altomonte u.a. (2013) hervor, dass sich die Internationalisierung nicht auf den Güterhandel beschränkt, sondern auch Auslandsdirektinvestitionen und Outsourcing umfasst. Sie zeigen, dass F\&E- und 
Innovationstätigkeiten das Ausmaß der Internationalisierung der Unternehmen erhöht. Aus diesem Grund sollten auch Maßnahmen zur Exportförderung darauf abzielen, die Innovationsfähigkeit einheimischer Unternehmen (etwa durch steuerliche Anreize oder Förderungen) zu erhöhen, da auf diese Weise die Internationalisierung intensiviert wird. Fritsch und Görg (2015) kommen in Bezug auf 28 Ökonomien Mittel- und Osteuropas sowie Zentralasiens $\mathrm{zu}$ einem vergleichbaren Ergebnis: Outsourcing und Importe durch Unternehmen erhöhen die Innovationstätigkeit.

\subsubsection{Produktivitäts- und Exportentwicklung in Österreich}

Trotz steigender Forschungsquote und einer relativen Verbesserung des BIP je Einwohner steht der Wirtschaftsstandort zunehmend unter Kritik. Eine Reihe von Indikatoren weist in den letzten Jahren auf eine sinkende Wettbewerbsfähigkeit hin. Reduziert man die Darstellung aus Abbildung 7 in Abbildung 22 auf den Zeitraum ab 2010, dann hinkt Österreich dem Produktivitätswachstum der meisten Innovation Leader und auch der EU als Ganzes hinterher. Ein Abstand von ein bis zwei Prozentpunkten über einen Zeitraum von fünf Jahren ist zwar nicht sehr groß; ein kumulatives Produktivitätswachstum von ca. $3 \%$ in fünf Jahren ist allerdings auch nicht gerade hoch. Entscheidend ist, dass sich dieser Trend nicht verfestigt und die F\&E-Investitionen wieder mehr Ertrag entfalten.

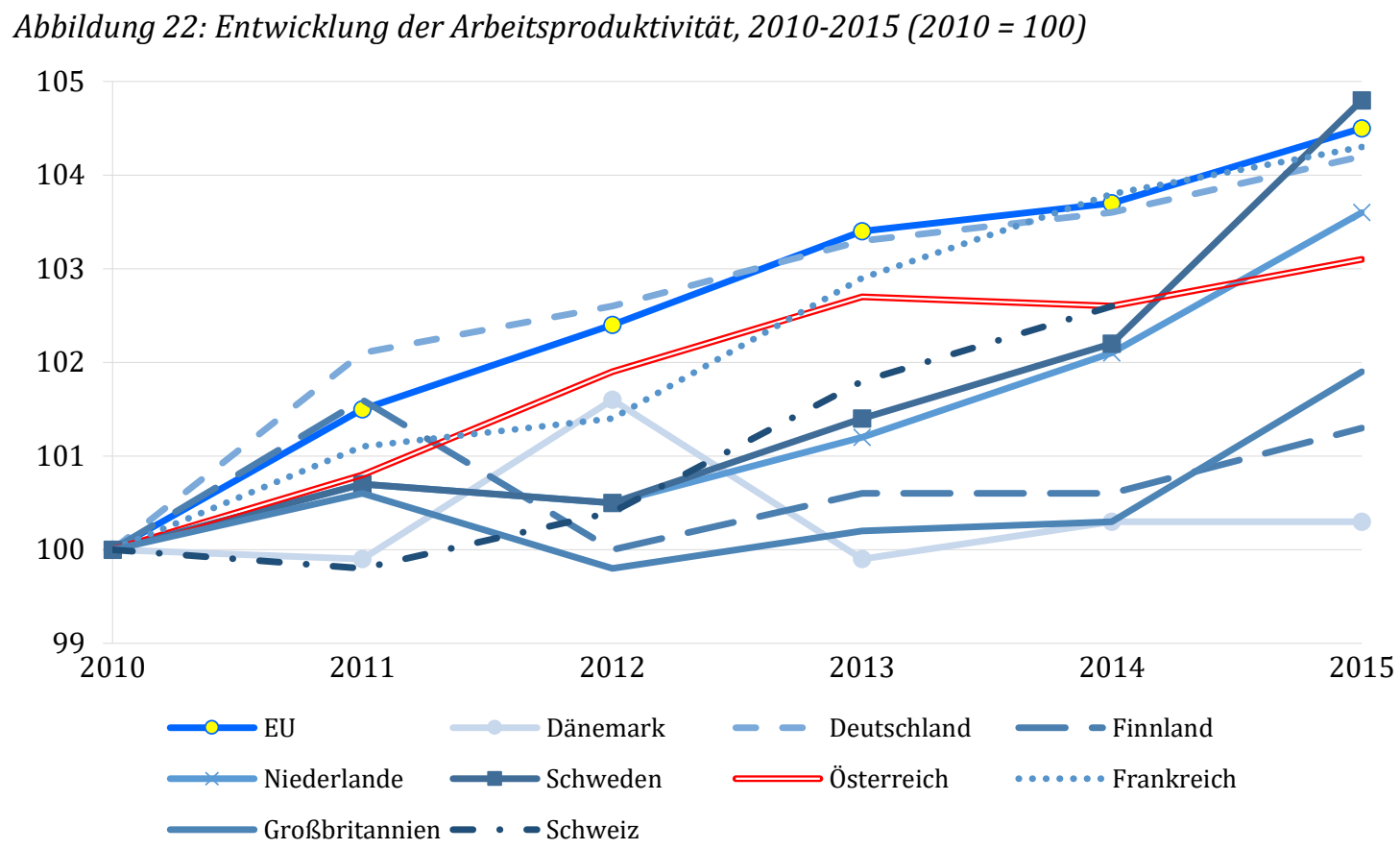

Quelle: Eurostat, eigene Berechnung und Darstellung

Das verlangsamte Produktivitätswachstum fällt mit Anzeichen einer Schwäche auf den Exportmärkten zusammen. Zwar erzielt Österreich regelmäßig einen Überschuss in der Leistungsbilanz und konnte das Land seine Exportquote von 33,6\% des BIP im Jahr des EUBeitritts 1995 auf 53,1\% im Jahr 2015 erhöhen. Allerdings erreichte die Exportquote bereits in 2006 einen Wert von 50,8\% und hat sich diese seither kaum noch verbessert. Ein 
Leistungsbilanzüberschuss bedeutet zunächst nur, dass ein Land mehr exportiert als importiert, sagt aber noch nichts darüber aus, welche Güter und zu welchem Preis gehandelt werden. Die Zusammensetzung der Exporte und Importe zeigt die Stärken und Schwächen und damit die komparativen Vorteile einer Wirtschaft.

Tabelle 11: Absolute und komparative Handelsvorteile Österreichs, 2014

\begin{tabular}{|c|c|c|c|c|}
\hline $\begin{array}{l}\text { SITC-Kode } \\
\text { (Rev. 3) }\end{array}$ & Bezeichnung & $\begin{array}{l}\text { Importe } \\
\text { (Tsd. €) }\end{array}$ & $\begin{array}{l}\text { Exporte } \\
\text { (Tsd. €) }\end{array}$ & RCA \\
\hline 51 & Organische chemische Erzeugnisse & 1.897 & 970 & $-65,48$ \\
\hline 52 & Anorganische chemische Erzeugnisse & 994 & 430 & $-82,18$ \\
\hline 53 & Farbmittel, Gerbstoffe und Farben & 1.019 & 714 & $-33,94$ \\
\hline 54 & Medizinische und pharmazeutische Erzeugnisse & 9.629 & 11.233 & 17,01 \\
\hline 55 & $\begin{array}{l}\text { Ätherische Öle, Körperpflege-, Putz-, Polier -, } \\
\text { Reinigungsmittel }\end{array}$ & 1.758 & 1.000 & $-54,84$ \\
\hline 56 & Düngemittel (ausgenommen solche der Gruppe 272) & 277 & 53 & $-163,93$ \\
\hline 57 & Kunststoffe in Primärformen & 3.205 & 2.499 & $-23,26$ \\
\hline 58 & Kunststoffe in anderen Formen als Primärformen & 2.049 & 2.500 & 21,50 \\
\hline 59 & Chemische Erzeugnisse und Waren, a.n.g. & 2.384 & 1.871 & $-22,65$ \\
\hline 61 & Leder, Lederwaren, a.n.g., und zugerichtete Pelzfelle & 378 & 706 & 64,14 \\
\hline 62 & Kautschukwaren, a.n.g. & 1.618 & 748 & $-75,49$ \\
\hline 63 & Kork- und Holzwaren (ausgenommen Möbel) & 1.176 & 3.053 & 97,04 \\
\hline 64 & $\begin{array}{l}\text { Papier und Pappe; Waren aus } \\
\text { Papierhalbstoff/Papier/Pappe }\end{array}$ & 2.615 & 5.022 & 66,86 \\
\hline 65 & Garne, Gewebe, fertiggestellte Spinnstofferzeugnisse, a.n.g. & 2.226 & 2.152 & $-1,77$ \\
\hline 66 & Waren aus nicht-metallischen mineralischen Stoffen, a.n.g. & 2.534 & 2.484 & $-0,39$ \\
\hline 67 & Eisen und Stahl & 4.715 & 9.037 & 66,66 \\
\hline 68 & NE-Metalle & 4.249 & 3.847 & $-8,36$ \\
\hline 69 & Metallwaren, a.n.g. & 7.167 & 8.850 & 22,69 \\
\hline 71 & Kraftmaschinen und Kraftmaschinenausrüstungen & 4.886 & 8.904 & 61,61 \\
\hline 72 & Arbeitsmaschinen für besondere Zwecke & 4.882 & 9.569 & 68,89 \\
\hline 73 & Metallbearbeitungsmaschinen & 1.100 & 1.938 & 58,24 \\
\hline 74 & Maschinen, Apparate und Geräte, a.n.g. & 9.335 & 10.591 & 14,22 \\
\hline 75 & $\begin{array}{l}\text { Büromaschinen, automatische Datenverarbeitungs- } \\
\text { maschinen }\end{array}$ & 3.238 & 1.929 & $-50,20$ \\
\hline 76 & $\begin{array}{l}\text { Geräte Nachrichtentechnik; Bild- und } \\
\text { Tonaufzeichnungsgeräte }\end{array}$ & 4.299 & 3.787 & $-11,08$ \\
\hline 77 & Elektrische Maschinen, Geräte, Einrichtungen, a.n.g. & 10.527 & 11.521 & 10,61 \\
\hline 78 & Straßenfahrzeuge (einschliesslich Luftkissenfahrzeuge) & 16.868 & 14.709 & $-12,10$ \\
\hline 79 & Andere Beförderungsmittel & 2.002 & 3.559 & 59,16 \\
\hline 81 & $\begin{array}{l}\text { Vorgefertigte Gebäude; Sanitäranlagen, Heizung, } \\
\text { Beleuchtung }\end{array}$ & 1.476 & 2.009 & 32,39 \\
\hline 82 & Möbel; Bettausstattungen, Sprungrahmen, ähnliche Waren & 2.591 & 1.512 & $-52,27$ \\
\hline 83 & Reiseartikel, Handtaschen und ähnliche Behältnisse & 533 & 129 & $-140,19$ \\
\hline 84 & Bekleidung und Bekleidungszubehör & 6.348 & 2.700 & $-83,87$ \\
\hline 85 & Schuhe & 1.966 & 1.052 & $-60,97$ \\
\hline 87 & Mess-, Prüf- und Kontrollinstrumente, a.n.g. & 2.951 & 3.246 & 11,14 \\
\hline 88 & $\begin{array}{l}\text { Fotoapparate, Zubehör, optische Waren, a.n.g.; } \\
\text { Uhrmacherwaren }\end{array}$ & 1.089 & 788 & $-30,69$ \\
\hline 89 & Verschiedene bearbeitete Waren, a.n.g. & 8.155 & 7.646 & $-4,84$ \\
\hline
\end{tabular}

Anmerkung: „a.n.g. " bezeichnet in der Beschreibung von Zolltarifnummern „anderweitig nicht genannt“ Quelle: OECD (www.keepeek.com/Digital-Asset-Management/oecd/trade/austria_itcs-v2015-4-2-en\#. V_UXQ4VOKUk\#page12), berechnet wie im Text dokumentiert 
Der industrielle Schwerpunkt in mittleren Technologien spiegelt sich auch in der Exportstatistik wider (Tichy, 2015). Über Jahrzehnte betrachtet hat sich zwar das technologische Niveau der Exportwirtschaft erhöht. Einmaleffekte wie der EU-Beitritt und die Ostöffnung haben die Nachfrage nach österreichischen Produkten belebt. Der relative Technologiegehalt der Exporte blieb allerdings konstant, sodass Österreich nicht zu anderen exportierenden Ländern technologisch aufholen konnte (Reinstaller, 2014). Da immer mehr aufstrebende Länder in die mittleren Technologiesegmente vorstoßen, wird dort die Konkurrenz intensiver und es fällt schwerer, eine hohe Wertschöpfung zu erzielen. Es scheint in Österreich nicht zu gelingen, sich vom mittleren Technologiesegment abzusetzen und an die Technologiegrenze vorzustoßen. Seit der Jahrtausendwende findet das Exportwachstum ganz überwiegend am intensiven Rand durch Mengenausweitung im Rahmen bereits bestehender Exportbeziehungen statt (Türkcan, 2014, und Stöllinger, 2015), und weniger am extensiven Rand durch Aufbau neuer Exportbeziehungen und Erschließung neuer Exportmärkte. Diese Entwicklung setzt dem Exportwachstum Grenzen, weil die traditionellen Exportmärkte in Westeuropa über längere Zeit nur schwach wachsen dürften.

Ein Aufholen zur Technologiegrenze und ein Aufstieg zum Innovation Leader würden dagegen eher zu neuen Exportbeziehungen führen und vor allem die komparativen Vorteile in den technologieintensiven Branchen stärken. In diesem Zusammenhang zeigt Türkcan (2014) ein Wachstum des extensiven Rands in Bezug auf wachsende Märkte wie China und Indien bzw. allgemein in den Extra-EU-Raum. Generell ergeben sich von 1998 bis 2011 höhere ExportWachstumsraten in aufstrebende Märkte - darunter auch die NMS - und relativ niedrige Export-Wachstumsraten in die als Innovation Leader klassifizierten Länder. Tabelle 11 zeigt die Handelsvorteile Österreichs.

Die komparativen Vorteile und damit die Stärken und Schwächen der Wirtschaft lassen sich mit dem sogenannten RCA-Index (Revealed Comparative Advantage, auch als Balassa-Index bezeichnet) darstellen, der Importe und Exporte zur aggregierten Außenhandelsposition in Beziehung setzt:

$$
R C A=100 \cdot \ln \left(\frac{X_{k} / \sum X_{k}}{M_{k} / \sum M_{k}}\right)
$$

Es bezeichnen $X_{k}$ die Menge an Exporten in der Branche $k$ und $M_{k}$ die Menge an Importen. Der Zähler im Klammerausdruck gibt den Anteil der Branche $k$ an den gesamten Exporten wieder, der Nenner steht für den Anteil der Branche an den gesamten Importen. Wenn der Exportanteil den Importanteil der Branche übersteigt und der Bruch einen Wert größer als eins annimmt, dann trägt die Branche relativ mehr zu den Exporten des Landes als zu den Importen bei. Der Index wird positiv und zeigt damit einen komparativen Vorteil an. Negative Werte entsprechen einem komparativen Nachteil im entsprechenden Sektor.

Tabelle 11 listet die komparativen Vorteile Österreichs in den wichtigsten Handelsbranchen auf. Komparative Nachteile sind vorwiegend bei chemischen Erzeugnissen in der Gruppe 5, und zwar in allen Untergruppen mit Ausnahme „medizinischer und pharmazeutischer Erzeugnisse“ (Untergruppe 54) sowie „Kunststoffe in anderen Formen als Primärformen“ (58), zu verzeichnen. Komparative Nachteile weisen auch die nicht sehr technologieintensiven Warengruppen 6 und 8 auf. Demgegenüber bestehen Vorteile in wichtigen Kategorien der Gruppe 7, Maschinenbauerzeugnisse und Fahrzeuge. Auch hier 
zeigt sich, dass Österreich über Vorteile im mittleren Technologiesegment verfügt. Damit ist aber das Risiko verbunden, dass Mitteltechnologie-Produkte vor allem aus aufstrebenden Ländern wie den BRICS-Staaten wettbewerbsfähiger werden und diese Länder auf den Absatzmärkten zunehmend mit Österreich konkurrieren.

\subsubsection{F\&E und ausländische Direktinvestitionen}

Im Folgenden wird zunächst mit deskriptiver Statistik ein Überblick zur Standortwahl von F\&E-Aktivitäten gegeben. Welche Standortfaktoren begünstigen ausländische Direktinvestitionen (FDI - Foreign Direct Investment) von forschungsintensiven Unternehmen? Sind F\&E und Produktion räumlich aneinander gekoppelt? Wie weit ist in der internationalen Wertschöpfungskette eine räumliche Aufspaltung und Fragmentierung von F\&E und Produktion möglich? Und zuletzt, findet neuerdings ein Reshoring, also eine Rückverlagerung von Produktionsaktivitäten aus Schwellenländern in reiche Länder statt?

Ein immer größerer Teil des internationalen Handels findet innerhalb multinationaler Unternehmen (Multis) anstatt zwischen unabhängigen Handelspartnern statt. Um Kosten zu senken, haben die Multis Produktionskapazitäten in Schwellenländern aufgebaut und zunächst arbeitsintensive und später auch komplexere Produktionsschritte verlagert. Auch die Leitbetriebe-Standortstrategie des BMWFW weist darauf hin, dass Leitbetriebe, d.h. wettbewerbsfähige international ausgerichtete Großunternehmen, regelmäßig und kritisch ihre Investitionsstandorte analysieren. Dabei wird in der Regel 10 bis 15 Jahre im Voraus geplant, weswegen der langfristigen Berechenbarkeit der Standortpolitik eine hohe Bedeutung zukommt (BMWFW, 2014b). Mittlerweile internationalisieren die Multis auch zunehmend ihre F\&E-Aktivitäten, die bei weitem nicht mehr nur an den Hauptsitz der Multis in ihrem Heimatland gebunden sind (Colovic und Mayrhofer, 2011, und Sachwald, 2008). Die zunehmende Mobilität wirft in den Hochlohnländern die Frage nach der Standortattraktivität für F\&E-Funktionen auf (OECD, 2011, und Reiner, 2012).

Tatsächlich treiben zwei Motive die Internationalisierung von F\&E und verwandten wissensintensiven Dienstleistungen: Erstens bauen Multis ausländische F\&E-Kapazitäten auf, um von der Nähe zur Nachfrage und zur lokalen unternehmenseigenen Produktion zu profitieren und die Produkte und Prozesse an die lokalen Verhältnisse auf dem ausländischen Absatzmarkt anzupassen. Auf diesem Weg fließt Wissen vom Heimatland der Multis an die ausländischen Standorte. Zweitens können die Multis mit ihren F\&E-Standorten im Ausland leichter das dort lokal verfügbare Wissen und Know-how nutzen. Ein Beispiel wären die Investitionen von europäischen IT-Unternehmen in ein eigenes Forschungslabor in Silicon Valley, um von der dortigen Forschungsumgebung zu profitieren. Dabei fließen Know-how und Wissen vom ausländischen Standort in das Heimatland.

Abbildung 23 nutzt die fDi Markets Database, wohl die umfassendste Datenbasis zu diesem Thema, und zeigt die globale Verteilung ausländischer Direktinvestitionen in Forschung, Entwicklung, Design und Testen (RDDT) nach der Zahl der Investitionen von 2003 bis 2011. Dabei dominieren die Elektronikindustrie und die IKT-Dienstleistungsbranche mit mehr als 40\% an der Gesamtzahl an RDDT-Investitionsprojekten; Nordamerika, Europa und Asien sind die zentralen Akteure; Afrika und Lateinamerika spielen nur eine untergeordnete Rolle; und Asien ist der größte „Nettoempfänger" von wissensintensiven Investitionsprojekten. Sowohl Nordamerika als auch Europa investieren deutlich öfter in Asien als umgekehrt. Die USA und 
Kanada sind mit 1.192 RDDT Investitionsprojekten in Asien wesentlich aktiver als die Europäer mit nur 826. Zudem haben die USA und Kanada etwa doppelt so viele RDDTInvestitionen in Europa getätigt als umgekehrt. Die Bedeutung des europäischen Binnenmarkts zeigt sich auch daran, dass mit 640 Projekten wesentlich mehr Investitionen innerhalb Europas erfolgten als innerhalb Asiens (471) oder Nordamerikas (110).

Abbildung 23: Investitionsprojekte in Forschung, Entwicklung, Design und Testen (RDDT), 2003-2011

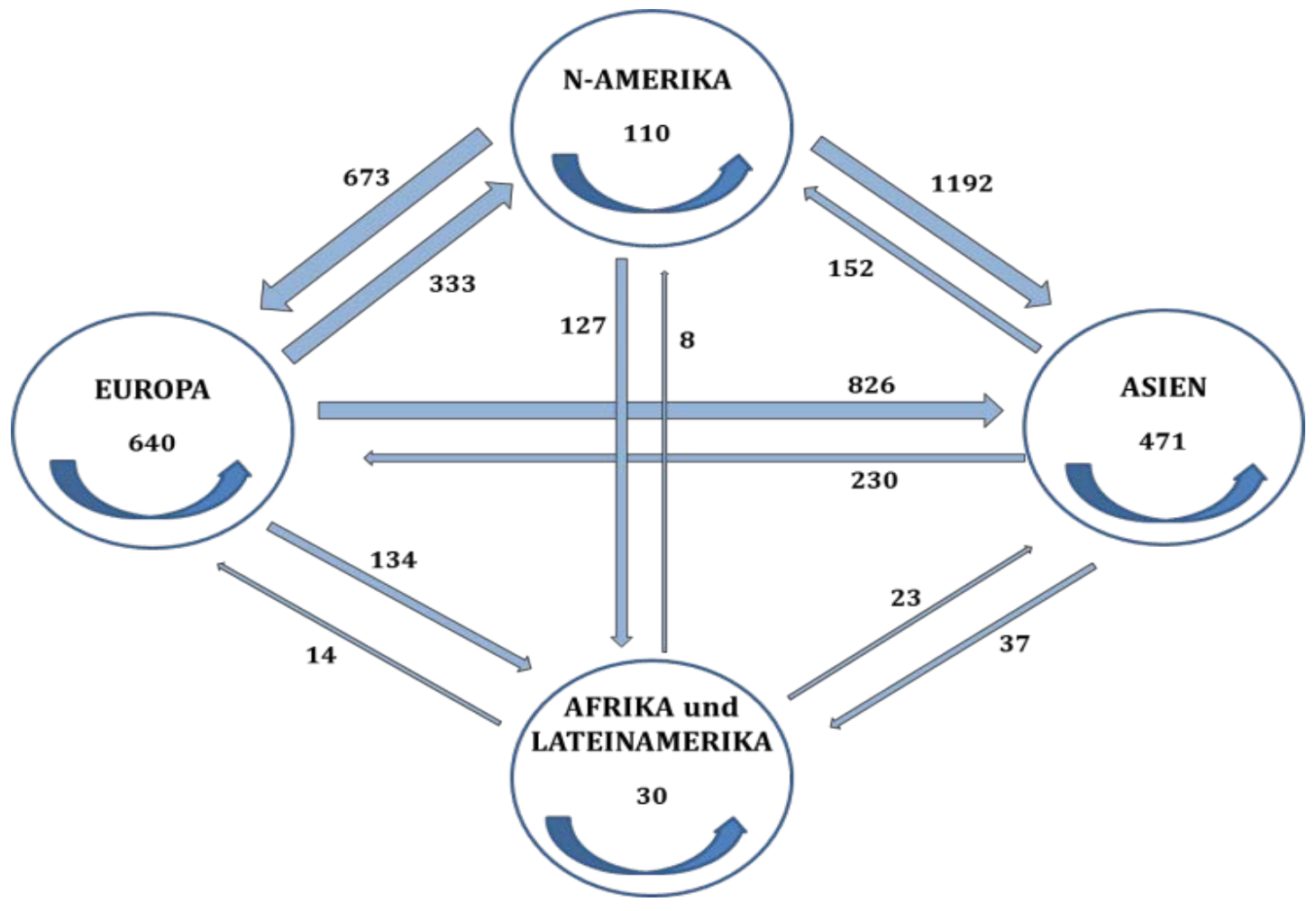

Anmerkung: Anzahl der Investitionsprojekte auf der grünen Wiese

Quelle: Belderbos u.a. (2016)

Europa tätigt demnach weniger F\&E-Investitionen auf ausländischen Wachstumsmärkten als die USA und dürfte daher auch weniger von externen Wissensquellen profitieren. Die Schätzungen von Belderbos u.a. (2016) zeigen, dass ausländische RDDT-Direktinvestitionen die wissensintensiven Aktivitäten am Heimatstandort anregen. Dachs und Ebersberger (2013) bestätigen dieses Ergebnis mit europäischen Daten aus sieben Ländern (inklusive Österreich) für das Jahr 2009. So führten 76\% der Unternehmen mit Offshoring-Aktivitäten von 2006 bis 2009 ein neues Produkt ein, während derselbe Anteil bei vergleichbaren Unternehmen ohne Offshoring-Aktivitäten bei 62\% lag. Der Unterschied von 14 Prozentpunkten ist höchst signifikant (Dachs und Ebersberger 2013, Tabelle 4-5). Von überragender Bedeutung sind die Globalen Städte, das sind urbane Agglomerationen, die nicht nur für die nationale Wirtschaft, sondern auch in der internationalen Arbeitsteilung eine wichtige Zentrumsfunktion ausüben. Etwa 40\% aller RDDT-Direktinvestitionen von 2003 bis 2011 fanden in nur 57 Städten statt (Belderbos u.a., 2016). 
Der R\&D Survey der EU von 2015 ermöglicht einen Ausblick auf die zu erwartende räumliche Verteilung von F\&E-Investitionen in den nächsten Jahren (Tübke u.a., 2015). Ausgehend von 149 europäischen Großunternehmen, die im EU Industrial R\&D Scoreboard von 2014 unter den Top 1.000 vertreten waren, investieren die Unternehmen etwa 25\% ihrer F\&EInvestitionen im Ausland, wobei Nordamerika (11 Prozentpunkte von ca. 25\%) und die VR China (6 Prozentpunkte) die beiden wichtigsten Empfängerregionen sind. Die erwarteten F\&E-Investitionen nehmen innerhalb der EU nur verhalten zu, während Indien und China deutliche Zuwächse erwarten können. Das erwartete F\&E-Investitionswachstum für die USA und Kanada liegt deutlich über dem europäischen Wert (+5,8\% versus 2,6\% jährlich für die nächsten 3 Jahre). In Folge dürfte daher auch der Anteil Europas an den F\&E-Investitionen weiter abnehmen, während jener für Indien, China und die USA zunimmt. Dieses Muster zeigte sich schon in den vergangenen $R \& D$ Surveys und scheint einen stabilen Trend darzustellen, der für Europa weiter schrumpfende F\&E-Anteile erwarten lässt.

Abbildung 24: Ausländischer Anteil an den privaten F\&E-Ausgaben in \%, 2003 und 2013

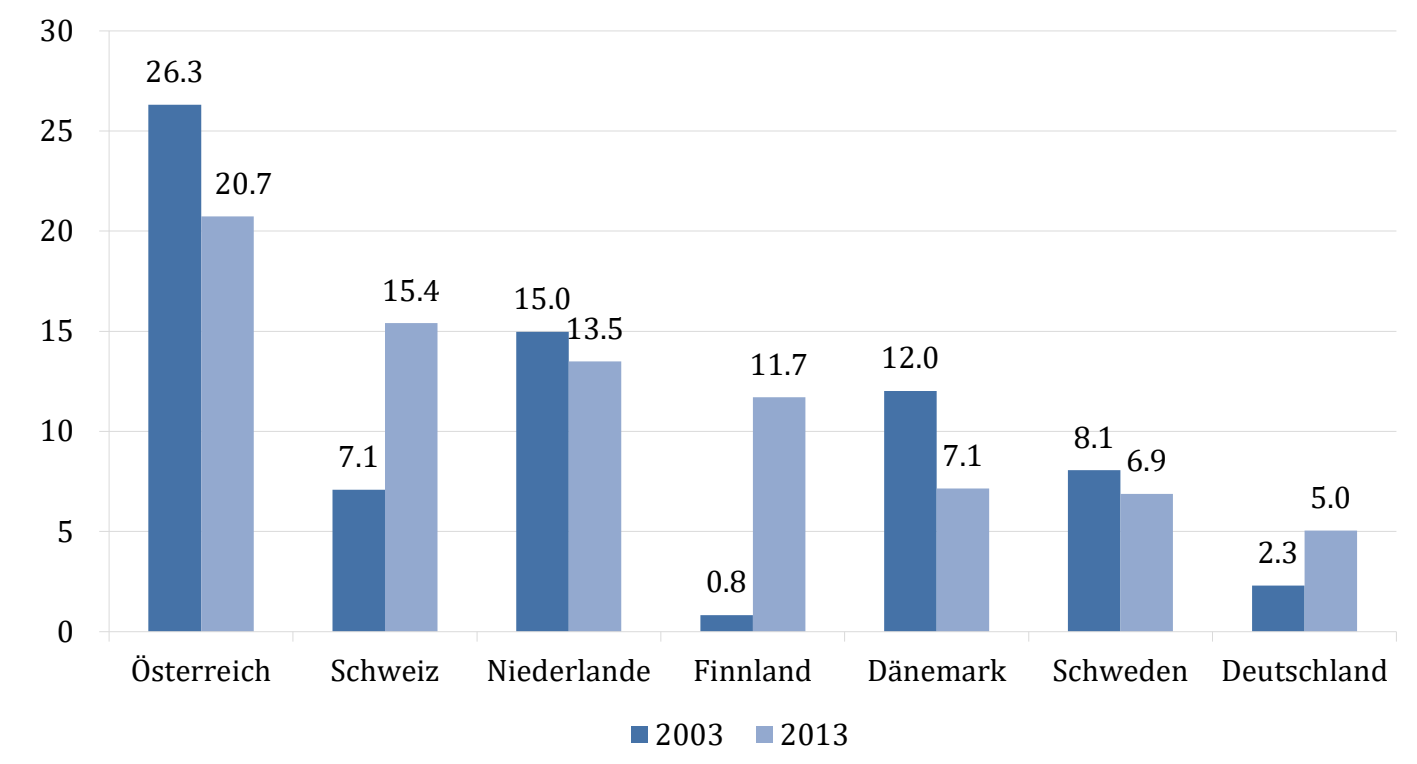

Anmerkung: Anteil des Auslands an den gesamten F\&E-Ausgaben des Unternehmenssektors (BERD) Quelle: Daten: OECD, MSTI

Österreich konnte die Internationalisierung der F\&E-Aktivitäten bislang gut nutzen (Gassler und Nones, 2008). Ein hoher Anteil an F\&E-Investitionen, die vom Ausland finanziert werden, deutet auf eine hohe Standortattraktivität für ausländische F\&E-Investitionen hin. Österreich weist in Abbildung 24 mit deutlichem Abstand den höchsten Anteil an ausländisch finanzierten F\&E-Investitionen des Unternehmenssektors auf. Auch wenn der Anteil seit 2003 von 26,3 auf 20,7\% gesunken ist, beträgt der Abstand zur Schweiz im Jahr 2013 noch immer 5 Prozentpunkte. Vorteilhaft schlagen sich die gut ausgebildeten Arbeitskräfte, die geographische Nähe zu den Heimatmärkten von wichtigen Multis in Deutschland, Italien und der Schweiz sowie die Nähe zu den NMS nieder. 
Der hohe Auslandsanteil an F\&E-Finanzierung zeigt eine hohe Standortattraktivität, aber zugleich auch eine gewisse Abhängigkeit des heimischen F\&E-Standorts auf. Die ausländischen F\&E-Einheiten in Österreich sind dabei relativ intensiv mit den heimischen Unternehmen und Universitäten vernetzt (Gassler und Nones, 2008), was die Bindung an den heimischen Standort verstärkt. Andererseits zeigen Håkanson und Kappen (2016) für ausländische F\&E-Einheiten in Schweden, dass die Gefahr einer Schließung mehr von ihrer Rolle innerhalb des multinationalen Konzerns als von den lokalen Standortfaktoren abhängt.

\subsubsection{Determinanten der F\&E-Standortwahl}

Je nach Branche, Art der Innovationsaktivität (Grundlagenforschung, angewandte Forschung, experimentelle Entwicklung) oder strategischer Orientierung (Nähe zum Absatzmarkt bzw. Produktionsstandort oder Nutzung lokaler F\&E-Kompetenz) stellen Multis unterschiedliche Ansprüche, die auf unterschiedliche F\&E-Standortvorteile treffen (Veliyath und Sambharya, 2011; Siedschlag u.a., 2013; OECD, 2011). Colovic (2010) nennt viele Standortfaktoren, die von der Qualität des Humankapitals, der Exzellenz der Grundlagenforschung über Agglomerationsvorteile, die Infrastruktur, die Marktgröße und das Marktwachstum bis hin zu der fiskalischen F\&E-Förderung und der Attraktivität des Finanzplatzes reichen.

Tabelle 12: Motive für F\&E-Aktivitäten von deutschen Multis im Ausland (2011)

\begin{tabular}{lc}
\hline & wichtig oder sehr wichtig \\
\hline Markterschließung, z.B. Produktanpassung für den ausländischen Markt & $57 \%$ \\
$\begin{array}{l}\text { Erschließen von spezifischem Wissen oder Fachkräften im Zielland, das bzw. die } \\
\text { in Deutschland nicht vorhanden sind }\end{array}$ & $55 \%$ \\
Einsparungen bei Löhnen und Lohnnebenkosten & $34 \%$ \\
Finanzierungsvorteile, z.B. staatliche F\&E-Förderungen, Steuervorteile & $22 \%$ \\
Politische Auflagen der Regierung im Zielland, z.B. Forderung nach F\&E- & $8 \%$ \\
Tätigkeit als Voraussetzung für Marktzugang & \\
Schwächere regulatorische Auflagen im Zielland, z.B. schwächere & $2 \%$ \\
Umweltschutzauflagen, geringere ethische Einschränkungen der Forschung & \\
\hline
\end{tabular}

Quelle: Czernich (2014)

Tabelle 12 zeigt die Ergebnisse einer Befragung von 113 deutschen Unternehmen, die für etwa 50\% der gesamten Auslandsforschung deutscher Unternehmen verantwortlich sind. Als hauptsächliche Beweggründe liegen dabei mit 57\% bzw. 55\% der Nennungen Markterschließung durch lokale Produktanpassungen und die Nutzung von lokalem Knowhow fast gleichauf an erster Stelle. Bereits deutlich weniger wichtig sind lohnkostenorientierte Faktoren und fiskalische Vorteile (mit 34\% bzw. 22\% der Nennungen). Diese Befragungsergebnisse entsprechen weitgehend den vielzitierten Ergebnissen von Thursby und Thursby (2006) für amerikanische Unternehmen. Dabei wurden 246 USUnternehmen über ihre internationalen F\&E-Aktivitäten befragt. Demnach sind im Wesentlichen vier Faktoren für die Standortwahl ausschlaggebend: Marktpotenzial, Qualität des F\&E-Personals, Zusammenarbeit mit Universitäten und intellektuelle Eigentumsrechte. Thursby und Thursby (2006) und Abramovsky u.a. (2007) streichen vor allem die wichtige 
Rolle von Universitäten heraus. Generell zählt offenbar mehr die Qualität der F\&EInputfaktoren, während Kostenfaktoren eine eher untergeordnete Rolle spielen.

Abbildung 25 zeigt die Motive deutscher Unternehmen für die Standortwahl ihrer F\&EInvestitionen im Ausland. Fällt die Entscheidung für hochentwickelte Staaten, dann sind Lohnkosten wenig relevant. Vielmehr gibt der Zugang zu spezifischem Wissen den Ausschlag. Die Lohnkosten sind dagegen sehr wohl entscheidend, wenn die Wahl auf weniger entwickelte Regionen fällt. Deshalb sind auch die F\&E-Aktivitäten in den BRIC-Staaten andere als jene in den USA oder Europa. Während etwa in Europa Grundlagenforschung und angewandte Forschung dominieren, findet in den BRIC-Staaten vor allem experimentelle Entwicklung statt (Czernich, 2014).

\section{Abbildung 25: Motive für F\&E-Aktivitäten von deutschen Unternehmen im Ausland, in \%}

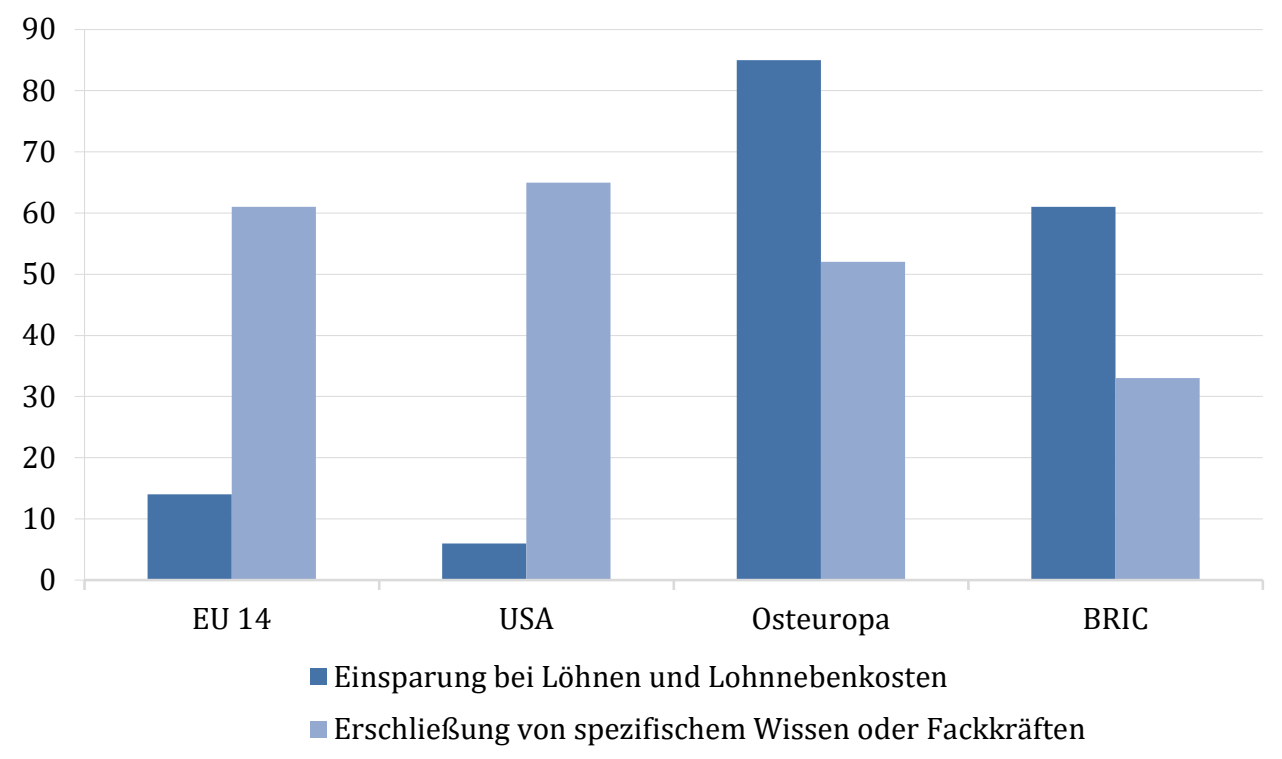

Anmerkung: In \% der Unternehmen, welche die Faktoren für wichtig bzw. sehr wichtig erachteten Quelle: Eigene Darstellung nach Czernich (2014)

Anders als die deskriptive Evidenz ergeben die Schätzungen von Belderbos u.a. (2016), dass Kosten in der späteren Phase der Standortsuche sehr wohl eine Rolle auch in hochentwickelten Ländern spielen, insbesondere wenn die anderen Standortfaktoren gut ausgeprägt sind. Des Weiteren zeigen die Ergebnisse, dass die Knappheit und die hohen F\&ELohnkosten einen wichtigen Push-Faktor für die Verlagerung von F\&E ins Ausland darstellen. Ein Anstieg der Lohnkosten in einer Stadt (die Untersuchung von Belderbos u.a. 2016 verwendet Daten auf regionaler Ebene) um 10\% reduziert die Wahrscheinlichkeit für eine ausländische Direktinvestition in den Bereichen Forschung, Entwicklung, Design oder Testen (RDDT) um ca. 9\%. Die Schätzungen von Lewin u.a. (2009) bestätigen dieses Ergebnis.

Eine weitere empirische Arbeit, wie jene von Siedschlag u.a. (2013), untersucht die Einflussfaktoren auf die F\&E-Standortwahl von multinationalen Unternehmen zwischen 1999 und 2006, wobei die Konzerne ihren Hauptsitz in den USA und in Europa haben. Im Gegensatz zu anderen Studien trägt die Arbeit der hohen Heterogenität innerhalb von 
Ländern Rechnung und betrachtet nicht Staaten, sondern Regionen als Investitionsstandorte. Es stehen also 233 europäische Regionen als mögliche Standorte für 446 Investitionsprojekte zur Verfügung. Allerdings unterscheidet die Arbeit mangels detaillierterer Daten nicht nach den verschiedenen Formen von F\&E. Es zeigt sich, dass die Wahrscheinlichkeit für eine F\&EAnsiedlung positiv auf die Agglomerationsdichte (Zahl ausländischer F\&E-Einheiten in der Region plus einer distanzgewichteten Zahl ausländischer F\&E-Einheiten in allen anderen Regionen), Verfügbarkeit von Humankapital, Nähe zu exzellenten Forschungseinrichtungen sowie Forschungs- und Innovationskapazitäten reagiert. Keinen signifikanten Einfluss haben hingegen Marktpotenzial, Lohnniveau, Arbeitslosenquote und Unternehmenssteuersätze. Die Elastizitäten geben jeweils die Veränderung relativ zum Durchschnittswert der Variablen an. Wenn demnach der Anteil der tertiär ausgebildeten Erwerbsbevölkerung um einen Prozentpunkt steigt (ausgehend vom Mittelwert von 23\%), nimmt die Wahrscheinlichkeit für eine ausländische F\&E-Direktinvestition in dieser Region um 0,6\% zu. Darüber hinaus können Regionen mit einer Top-500-Universität nach dem QS World University Ranking im Vergleich zu anderen Regionen ohne eine solche Universität eine um 1,4\% höhere Wahrscheinlichkeit für eine ausländische F\&E-Investition verbuchen. Wenn die Agglomerationsdichte (ausgehend vom Mittelwert 14, d.h. es befinden sich ca. 14 ausländische F\&E-Einheiten in der Region bzw. in deren Nähe) um eine Einheit steigt, nimmt die Wahrscheinlichkeit für eine ausländische F\&E-Investition um 0,035\% zu.

\subsubsection{Räumliche Koppelung von F\&E und Produktion}

Pisano und Shih (2009) erörtern die Rolle der Industrie für das nationale Innovationssystem der USA. Die Entwicklung einer wettbewerbsfähigen Industrie hängt dabei von einer kritischen Größe der industriellen Basis („industrial commons“) ab. Diese Basis besteht aus dem gleichzeitigen Vorhandensein von Kompetenzen in F\&E, Ingenieurswesen und in Produktionsprozessen, die komplementär zueinander sind. Die Kompetenzen sind einerseits auf verschiedene Akteure wie Universitäten, Unternehmen und spezialisierte Dienstleister verteilt und andererseits räumlich konzentriert. Komplementarität heißt, dass die Rentabilität der Innovation umso höher ist, je mehr F\&E-intensive Produktionsprozesse angesiedelt sind, und umgekehrt. Ähnlich wie bei Netzwerken gibt es eine gegenseitige Verstärkung, so dass eine ausreichende Profitabilität erst beim Erreichen einer kritischen Größe erzielt wird. Umgekehrt könnte eine Erosion der industriellen Basis eine negative Kettenreaktion auslösen, wenn komplementäre Ressourcen für Innovationsprozesse verloren gehen. Mit der umfangreichen Produktionsverlagerung ins Ausland (z.B. in der Elektronikindustrie) haben nach Pisano und Shih (2009) die USA bereits wesentliche Voraussetzungen für die zukünftige Entwicklung von High-Tech-Branchen verloren. Die Ansicht, die USA könnten sich alleine auf F\&E, Design und Marketing spezialisieren, sei eine völlig falsche Vorstellung von Innovationsprozessen. Aghion u.a. (2011) betonen eine weitere Ursache für den engen Zusammenhang von Produktion und Innovation: Der Transfer von Forschungsergebnissen in die Fabrik und ihre industrielle Anwendung tragen entscheidend zur Entwicklung technologischer Fähigkeiten bei, welche über positive externe Effekte wie z.B. Arbeitskräftemobilität auch für andere Unternehmen verfügbar werden.

Die Abwanderung reifer Industrien in kostengünstige Länder ermöglicht zwar eine internationale Arbeitsteilung, allerdings kann dieser Prozess auch negative Konsequenzen haben, wenn damit komplementäre Elemente der industriellen Basis verloren gehen. Nach 
Pisano und Shih (2009) ist die Entstehung neuer Industriezweige bisweilen von einer hinreichenden Präsenz reifer Industrien als Teil der industriellen Basis abhängig. So habe die Abwanderung der Halbleiterindustrie die Voraussetzungen für eine erfolgreiche Entwicklung von Solarzellen entscheidend beeinträchtigt, weil die Entwicklung und Produktion von Solarzellen Kompetenzen in der Halbleiterproduktion voraussetzt.

Mit der internationalen Aufspaltung der Wertschöpfungsketten und der Verteilung von Unternehmensfunktionen auf Standorte mit komparativen Vorteilen stellt sich damit auch die Frage, welche Funktionen räumlich gekoppelt sind und besser gemeinsam angesiedelt werden. Empirische Untersuchungen zeigen, dass die Produktion die stärkste Anziehungskraft für andere Unternehmensfunktionen aufweist (OECD, 2011). Insbesondere neigen Unternehmen dazu, „Design und Entwicklung“ in räumlicher Nähe zur Produktion anzusiedeln. Aber auch die Standortwahl von F\&E-Funktionen hängt mit dem Produktionsstandort zusammen. Befunde aus der Regionalökonomie zeigen, dass der Aufbau einer industriellen Basis die Ansiedlung von hochwertigen produktionsnahen Dienstleistungen begünstigt. Regionen, die noch nicht als Zentren für hochwertige Dienstleistungen etabliert sind, haben ohne industrielle Basis meist geringe Chancen, einen wissensintensiven Dienstleistungssektor zu entwickeln (Paqué, 2009).

Die Vorteile der räumlichen Nähe von Produktion und F\&E bedeuten jedoch noch nicht, dass eine Produktionsverlagerung automatisch die Wahrscheinlichkeit einer Abwanderung der F\&E steigert. Belderbos (2016) kann einen solchen „Push-Effekt“ nicht feststellen. Ist jedoch eine Entscheidung zur Verschiebung von F\&E-Aktivitäten gefallen, dann siedeln die Firmen mit höherer Wahrscheinlichkeit die F\&E an jenem Standort an, der zuvor als Produktionsstandort gewählt wurde. Dachs und Ebersberger (2013) werten Daten des European Manufacturing Surveys von 2009 für 3.106 Unternehmen aus und zeigen, dass eine Produktionsverlagerung ins Ausland nicht nur keine negativen Effekte auf heimische Innovationsaktivitäten hat, sondern diese im Gegenteil sogar verstärken kann. Für die USA stellen Bilir und Morales (2016) ähnliche Zusammenhänge fest. Nach Daten von 1989 bis 2008 investieren etwa 92,5\% der amerikanischen Muttergesellschaften in F\&E, aber nur $47,8 \%$ haben mindestens eine ausländische Filiale, die auch in F\&E investiert. Der Anteil der Auslandstöchter an den F\&E-Ausgaben der Konzerne macht nur 8,5\% aus. Die F\&E am Konzernsitz stärkt die Marktposition und Profitabilität der ausländischen Standorte und ist zur lokalen F\&E komplementär. Ein Anstieg um 1\% der F\&E-Ausgaben der Mutter wirkt etwa vier Mal so stark auf die Wertschöpfung der Tochter wie ein 1 prozentiger Anstieg der F\&EAusgaben der Tochter selbst. Die F\&E-Ausgaben der Mutter sind umso wirksamer, je mehr eine Auslandstochter selbst F\&E-aktiv ist. Würden die Vorteile der F\&E-Ausgaben am Stammsitz wegfallen, würden bei sonst gleichen Bedingungen die Leistungen einer durchschnittlichen Tochtergesellschaft langfristig um 58\% abnehmen.

\subsubsection{Rückkehr der Industrie aus dem Ausland}

Die Verlagerung der Produktion von den Industriestaaten in die lohngünstigen Entwicklungsund Schwellenländer hat lange Zeit die Globalisierung geprägt. Seit Beginn des Jahrzehnts ist jedoch vereinzelt eine Umkehr der Produktionsverlagerung zu beobachten. Eine solche Heimkehr der Industrie („Reshoring“) könnte insbesondere in den USA (Tate, 2014) und in Großbritannien (Baileya und De Propris, 2014) eine teilweise Re-Industrialisierung einleiten, 
da diese Länder zuvor besonders stark von einer De-Industrialisierung betroffen waren. 21 Letztlich ergibt sich dieser Trend aus einer Überprüfung der Standortentscheidungen in einer sich stets ändernden Welt (Gray u.a., 2013), um die Produktion wieder dort anzusiedeln, wo die Standortbedingungen den neuen technologischen und institutionellen Anforderungen am ehesten entsprechen. So wurden in der Vergangenheit mitunter standardisierte Produktionsschritte mit vielen Routineaufgaben und hohen Lohnkosten ausgelagert. Eine Auslagerung ist jedoch nicht mehr notwendig, wenn solche Arbeitsplätze mit fortschreitender Automatisierung und dem Einsatz von Industrierobotern wegfallen.

Der Überblick von Ellram u.a. (2013) zählt eine Reihe von Gründen für die Rückkehr der Industrie auf, wie z.B. höhere Produktivität und niedrigere Lohnkosten pro Output-Einheit in den Industrieländern (Automatisierung), steigende Lohnkosten in Niedriglohnländern, wachsendes Umweltbewusstsein und steigende Umweltkosten in den Entwicklungsländern, steigende Treibstoff- und Transportkosten, Diebstahl intellektuellen Eigentums, Korruption, mangelnde Rechtssicherheit und andere institutionelle Defizite in den Entwicklungsländern. Zudem können die Unternehmen mit der Heimkehr der industriellen Produktion auch die Vorteile der örtlichen Nähe von F\&E und Produktion besser nutzen.

21 US-Präsident Obama und der britische Premierminister Cameron erklärten, eine solche Reindustrialisierung zu unterstützen, vgl. www.whitehouse.gov/the-press-office/2012/01/11/remarks-president-insourcing-american-jobs, Statement vom 11. 1. 2012, sowie The Economist, 4. 12. 2014. Auch Österreich könnte von einem „Reshoring“ profitieren, da heimische Unternehmen hohe Direktinvestitionen im Ausland getätigt und arbeitsintensive Teile der Wertschöpfungskette ausgelagert haben. 


\section{Wirtschaftspolitische Rahmenbedingungen}

Unternehmen, die neue Produkte entwickeln und diese als erste auf den Markt bringen, erschließen neue ergiebige Absatzquellen. Mit Prozessinnovationen können sie Kosten sparen, preisliche Wettbewerbsvorteile erlangen und ihre Marktposition ausbauen. Innovative Firmen sind daher profitabler, wachsen rascher und schaffen mehr Jobs und Einkommen. Eine hohe F\&E-Intensität befähigt die Unternehmen zudem, neue Entwicklungen in der Grundlagenforschung aufzugreifen und in kommerzielle Anwendungen zu übersetzen, und neue Technologien rascher von anderen Unternehmen zu übernehmen und weiterzuentwickeln (Jaffe, 1986; Griffith, Redding und Van Reenen, 2004).

\subsection{Warum Innovationsförderung?}

Der positive Einfluss von Innovation auf das Wachstum begründet jedoch nicht automatisch eine staatliche Förderung, denn Innovation hat auch Kosten. Der Staat ist nur dann gefordert, wenn Marktstörungen bestehen. Im Wesentlichen sind drei Gründe für Innovationsförderung zu nennen, nämlich externe Erträge, Kreditbeschränkungen und internationaler Systemwettbewerb. Erstens kann ein Teil der gesamten F\&E-Erträge an andere, externe Nutzer fließen, ohne dass diese dafür bezahlen. Wenn die Unternehmen zwar alle Kosten selber tragen, aber die Erträge mit anderen teilen müssen, dann ist die private Rendite von F\&E-Investitionen geringer als die gesamtwirtschaftliche. Daher investieren die Firmen systematisch zu wenig in F\&E. Mit Innovationsförderung sollen sie für die externen Erträge kompensiert werden, um Innovation anzuregen. Jones und Williams (1998) schätzen die private Rendite von F\&E auf 7 bis 14\% und die soziale Ertragsrate auf etwa 30\%. Die optimale Investitionsrate läge damit um zwei- bis viermal höher als die tatsächlichen F\&E-Ausgaben.

Ein zweiter Grund für Innovationsförderung sind Finanzierungsbeschränkungen. Rentable F\&E-Investitionen kommen nicht zustande, wenn externe Kapitalgeber keine Finanzierung geben. Junge innovative Wachstumsunternehmen haben großen Finanzierungsbedarf, aber auch ein hohes Risiko und können meist nur wenige Sicherheiten anbieten. Die Finanzierung von Banken ist damit begrenzt. Die Unternehmen weisen zudem erst eine kurze Geschichte auf und sind von außen schwer zu beurteilen. Mangelnde Gewissheit über ihren Zustand wecken Zweifel über die Sinnhaftigkeit eines Investments. Fehlendes Vertrauen schreckt auch die Risikokapitalgeber ab. Am Ende verfügen junge und innovative Unternehmen über sehr rentable Investitionsmöglichkeiten, die aber mangels Finanzierung nicht zum Zug kommen.

Drittens ist eine ausgebaute steuerliche F\&E-Förderung gerade für kleine Länder aus strategischen Gründen zur Positionierung im internationalen Standortwettbewerb ratsam. Wenn das Ausland großzügige F\&E-Förderung anbietet, sollte das Inland wenigstens nachziehen, um einen Wettbewerbsnachteil für heimische Unternehmen auszugleichen. Kleine Länder müssen die Standortattraktivität offensiver verteidigen, weil sie einen wichtigen Standortnachteil gegenüber großen Ländern kompensieren müssen, nämlich den kleinen heimischen Absatzmarkt.22 Die Besteuerung soll Standortattraktivität dort schaffen, wo es am dringendsten notwendig ist, nämlich für die mobilen Unternehmen, bei denen die Abwanderungsgefahr am größten ist. Exportfirmen und Multis sind die F\&E-intensivsten,

22 Vgl. Keuschnigg (2016) zum Zusammenhang zwischen der Höhe der Unternehmensbesteuerung und der Größe eines Landes. 
produktivsten, größten und mobilsten Unternehmen. Die steuerliche F\&E-Förderung hilft, die Entlastung der KÖSt auf jene Unternehmen zu beschränken, die besonders mobil sind. Sie vermeidet Mitnahmeeffekte einer allgemeinen und daher kostspieligen Steuersenkung, die alle Unternehmen entlastet, unabhängig davon, ob Standortüberlegungen eine Rolle spielen oder nicht. Da alle Firmen Anspruch auf die steuerliche F\&E-Förderung haben, gibt es keine Diskriminierung. Faktisch gesehen ist es dennoch eine gezielte und damit sparsame Maßnahme zur Stärkung der Standortattraktivität, weil die F\&E-Ausgaben statistisch bei den mobilen Unternehmen konzentriert sind und dort die Begünstigung überdurchschnittlich stark zu Buche schlägt.

\subsection{Patentschutz}

Die erste Vorkehrung, um den Unternehmen die Erträge aus ihren F\&E-Aufwendungen zu sichern, ist der Patentschutz (vgl. z.B. Hall, 2008, und Boldrin und Levine, 2008). Der Großteil der Patentanträge geht an die Patentämter der USA, der EU und Japans, wobei ein EU-Patent Schutz in allen angeschlossenen Mitgliedstaaten gewährt und so erhebliche Kosten spart. Typischerweise wird ein Patent für 20 Jahre vergeben und sichert die exklusive Nutzung durch die Erfinderin bzw. den Erfinder. Dabei kommt es allerdings nicht nur auf den rechtlichen Schutz, sondern auch auf die effiziente Durchsetzung der Patentrechte im Hinblick auf Kosten, Dauer und Rechtssicherheit bei Patentstreitigkeiten an. Die Androhung von teuren und langwierigen Patentprozessen durch finanzkräftige Konzerne kann auf kleine innovative Unternehmen auch abschreckend wirken und teilweise F\&E-Investitionen bremsen.

Abbildung 26 zeigt die Zahl der triadischen Patentfamilien pro Million Einwohner im Jahr 2013 im internationalen Vergleich.23 Österreich liegt zwar deutlich über dem OECDDurchschnitt und etwa gleichauf mit den Niederlanden, Dänemark, Schweden und Deutschland, während Japan und vor allem die Schweiz weit mehr als doppelt so viele Patente vorweisen können. Die Position der Schweiz dürfte neben den hohen Investitionen in der Grundlagenforschung wesentlich von der Präsenz vieler schweizerischer multinationaler Unternehmen mit besonders hoher Forschungsintensität abhängen.

Die Ziele des Patentschutzes sind der Schutz der privaten F\&E-Erträge und die Förderung der Innovation. Im Austausch für zeitlich begrenzte Exklusivrechte macht die Erfinderin bzw. der Erfinder die neuen Erkenntnisse öffentlich und erleichtert anderen die Weiterentwicklung. Volkswirtschaftlich hat der Patentschutz nicht nur Vorteile, sondern auch Nachteile. Patente ermöglichen eine zeitlich begrenzte Monopolstellung, sichern damit den Ertrag von privater F\&E und machen F\&E-Investitionen erst rentabel. Die zeitlich begrenzte Einschränkung des Wettbewerbs ist eine notwendige Voraussetzung für Innovation und Wachstum. Der Wettbewerb spielt dabei über die F\&E-Aufwendungen der anderen Mitbewerber, die mit besseren Leistungen bestehende Anwendungen und Produkte verdrängen. Wenn jedoch die Innovation ein kumulativer Prozess ist und die Weiterentwicklung eines neuen Patentes die Nutzungsrechte einer Vielzahl existierender Patente benötigt, kann ein zu weitgehender Patentschutz auch innovationshemmend wirken, weil er die Kosten der nachfolgenden

23 Triadisch heißt, dass ein Patent gleichzeitig in der EU, den USA und Japan registriert und geschützt ist. Solche Patente beziehen sich auf Innovationen mit einem besonders großen Potenzial für das Unternehmenswachstum. 
Innovationen in die Höhe treibt. Je umfangreicher der Patentschutz, desto mehr Neuerungen sind den anderen Unternehmen zur weiteren Nutzung vorenthalten und desto stärker ist die Marktmacht des Patentinhabers. Diese Vor- und Nachteile muss eine optimale Ausgestaltung hinsichtlich Dauer und Umfang des Patentschutzes gegeneinander abwägen.

Abbildung 26: Triadische Patentfamilien pro Mio. Einwohner, 2013

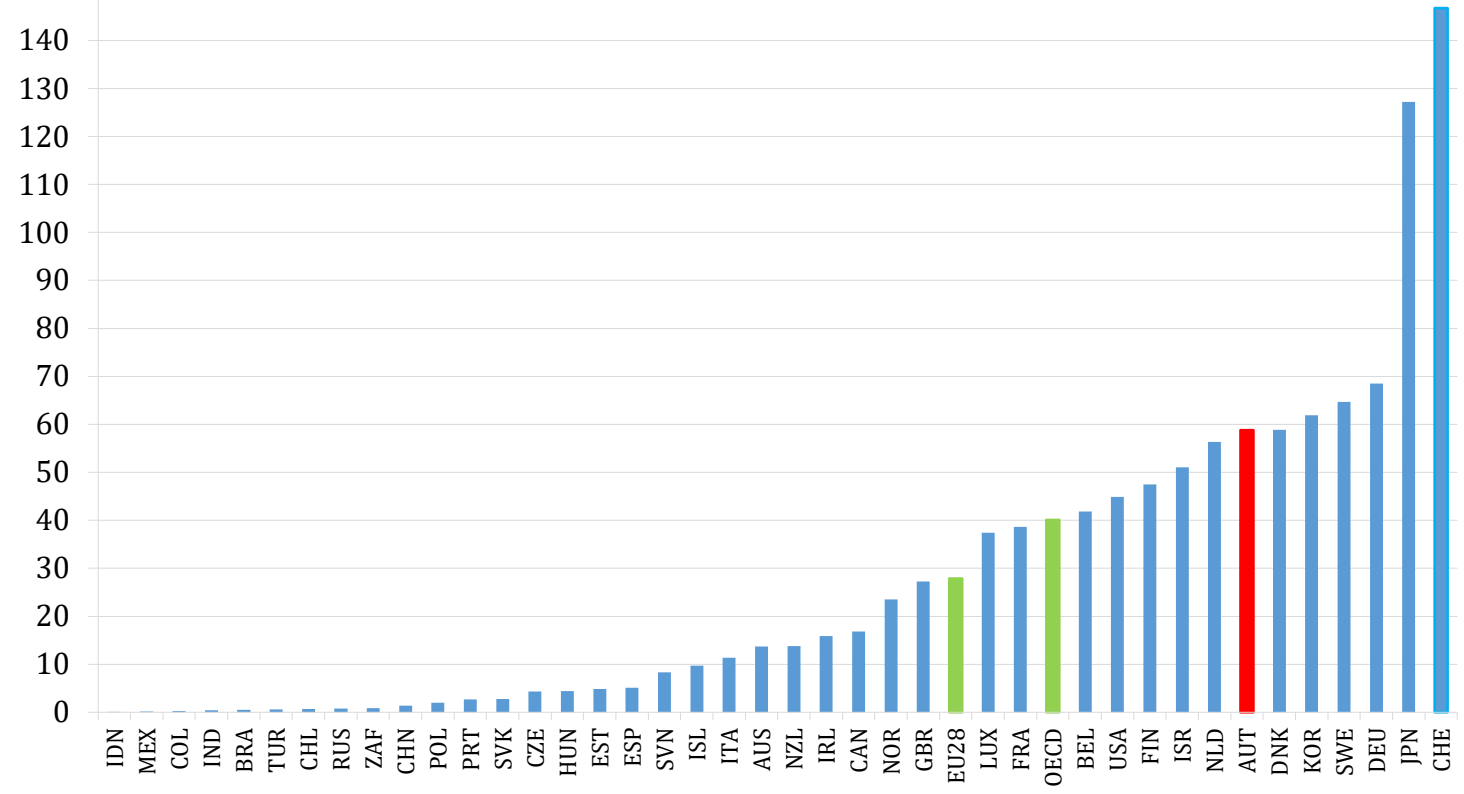

Quelle: OECD (2016), Triadic patent families (indicator). doi: 10.1787/6a8d10f4-en, Population (indicator). doi: 10.1787/d434f82b-en, eigene Berechnungen und Darstellung

Patente können die Unternehmensentwicklung stark beeinflussen. Farre-Mensa u.a. (2016) quantifizieren den kausalen Effekt einer Patentgewährung auf den nachfolgenden Erfolg einer Firma. Sie nutzen die Daten von 45.817 erstmaligen Patentanmeldungen von US-Startups von 2001 bis 2014, wovon 66\% bewilligt wurden. Im Median beschäftigten die Firmen etwa acht Angestellte und erzielten einen Umsatz von einer Mio. USD. Im Vergleich zu den Unternehmen, deren erstes Patentgesuch abgelehnt wurde, haben die erfolgreichen Firmen nach drei Jahren der Patentgewährung eine um 20,3 Prozentpunkte höhere Beschäftigung und einen um 22 Prozentpunkte höheren Umsatz. Nach fünf Jahren beträgt der Wachstumsunterschied 36 Prozentpunkte bei der Beschäftigung und 51,4 Prozentpunkte beim Umsatz. Ein Median-Start-up mit 8 Beschäftigten, dessen Patentgesuch bewilligt wurde, beschäftigt also nach fünf Jahren um 2,9 Beschäftigte mehr als ein vergleichbarer Start-up, dessen erster Patentantrag abgelehnt wurde. Eine erstmalige Patentbewilligung steigert nicht nur die Zahl, sondern auch die Qualität der nachfolgenden Patente. Relativ zur Vergleichsgruppe steigert die Patentgenehmigung die nachfolgenden Patentgesuche um $66,9 \%$ und die genehmigten Patente um 48,8\%. Die Zitationen pro bewilligtem Patent nehmen um $26,5 \%$ zu. 
Die Erteilung eines Patents beschleunigt also das Beschäftigungs- und Umsatzwachstum der Unternehmen, steigert ihre Innovationsleistung und stärkt ihre Chancen, mit einer Börseneinführung mehr Wertzuwachs zu generieren. Die Ergebnisse weisen zudem auf die hohe Bedeutung von Rechtssicherheit über die Eigentumsverhältnisse hin. In den USA beträgt z.B. die Dauer bis zum endgültigen Entscheid durchschnittlich 3,2 Jahre. Jedes zusätzliche Jahr, das die Patentbehörde für eine Entscheidung benötigt, schwächt das Beschäftigungswachstum nach drei bzw. fünf Jahren der Patentgewährung um 8,5 bzw. 21,2 Prozentpunkte. Ähnliches gilt für das Umsatzwachstum und die nachfolgende Innovationstätigkeit. Jedes Jahr an Verzögerung verringert die Zahl der nachfolgend eingereichten Patentgesuche um 13,1\% sowie die Zahl der bewilligten um 12,7\%. Auch die Qualität der eingereichten Patente leidet, denn dieselbe Verzögerung führt zu einem Rückgang in den Zitationen pro Patent um 7,1\%.

Warum wirken sich Patente so günstig auf die Unternehmen aus? Ein neu erteiltes Patent wirkt wie ein Qualitätssignal, baut die Unsicherheit externer Investoren über die weitere Unternehmensentwicklung ab und verbessert den Zugang zu Finanzierung. Tatsächlich steigt die Wahrscheinlichkeit, dass ein Start-up Risikokapital erhält, bereits ein Jahr nach der Patenterteilung um 1,2 Prozentpunkte, nach drei Jahren schon um 2,3 und nach fünf um 2,8 Prozentpunkte. Ein Anstieg um 2,3 Prozentpunkte bedeutet eine um 53\% höhere Wahrscheinlichkeit, drei Jahre nach der Patentgewährung Risikokapital zu erhalten. Die ForscherInnen zeigen außerdem, dass Patente die Kapitalbeschaffung besonders bei jenen Firmen erleichtern, die sehr jung sind, von unerfahrenen Unternehmern gegründet wurden, in Bundesstaaten mit vielen Start-ups angesiedelt und in der IT-Branche tätig sind.

Wenn eine Patenterteilung die Unternehmensentwicklung anstößt, dann sollte die Aberkennung eines Patents den umgekehrten Effekt bewirken. Galasso und Schankerman (2015) untersuchen 1.181 US-amerikanische Patentrechtsfälle von 1982 bis 2010, wobei 1.469 Patente betroffen waren. Manche Streitigkeiten betrafen mehrere Patente. Im Durchschnitt sprach das Bundesberufungsgericht in 40\% der Fälle eine Annullierung aus. Das Ergebnis ist, dass die Annullierung mindestens eines Patents innerhalb von fünf Jahren die Zahl der Patentanmeldungen der betroffenen Firma um 50\% senkt. Dieser negative Effekt stellt sich allerdings nur bei kleinen Firmen ein, nicht bei den großen. Zudem ist der Rückgang der Patenttätigkeit bei Firmen, welche das annullierte Patent als Sicherheit für Bankkredite genutzt hatten, etwa doppelt so groß wie bei anderen (Galasso und Schankerman, 2016). Der Verlust des Patentrechts steigert zudem die Wahrscheinlichkeit des Marktaustritts, aber wiederum nur bei kleinen Firmen. Bei Firmen mit einem Patentportfolio von weniger als 30 Patenten steigert ein Patentverlust die Austrittswahrscheinlichkeit um etwa 53\%. Bei Firmen mit weniger als drei Patenten steigt das Austrittsrisiko sogar auf $63 \%$ an.

Die Ergebnisse sind ein Hinweis darauf, dass kleine und innovative Unternehmen häufig mangelnden Zugang zu Kapital haben und ihre Wachstumsmöglichkeiten nicht ausschöpfen können, während große finanzkräftige Unternehmen kein Problem mit der Finanzierung haben. Indem Patente ein Qualitätssignal senden, Information und Vertrauen bei externen Kapitalgebern schaffen und als Sicherheit für Kredite zur Verfügung stehen, helfen sie innovativen KMU, mehr Finanzierung für die weitere Expansion aufzutreiben. Bei großen Unternehmen kommt dieser Effekt nicht zum Tragen, da sie die benötigte Finanzierung leicht 
erhalten. Aus diesem Grund können neue Patente die Entwicklung der KMU wesentlich kräftiger anstoßen als das weitere Wachstum der großen Konzerne.

\subsection{Grundlagenforschung}

Grundlagenforschung schafft neues Wissen, das wie ein öffentliches Gut ohne Rivalität von allen ForscherInnen nutzbar ist. Neue Einsichten und Ergebnisse bauen auf vergangenen Erkenntnissen auf. Die Ergebnisse der Grundlagenforschung sind kumulativ, sehr breit gefächert, unsicher und fallen oft mit großer Zeitverzögerung an. Spezifische Anwendungen ergeben sich erst im Laufe der weiteren Entwicklung und sind bisweilen überraschend und unvorhergesehen. Neues Wissen breitet sich rasch aus und wird allgemein bekannt. Deshalb fließen die Erträge weit über die investierende Forschungseinheit hinaus und nützen der gesamten Forschercommunity. Die soziale Ertragsrate der Grundlagenforschung ist wesentlich höher als die private Rendite. Unter diesen Umständen können sich private Firmen nur einen Bruchteil der Erträge aneignen und sind als Finanzierungsquelle oft nicht geeignet. Sie hätten auch kaum Anreize, die Verbreitung des neuen Wissens aktiv zu unterstützen, und würden damit den weiteren Fortschritt eher behindern. Aus diesem Grund ist die Finanzierung der Grundlagenforschung eine klassische Staatsaufgabe. Nach Gersbach u.a. (2015) entfallen im Durchschnitt der wichtigsten OECD-Länder etwa 22\% der gesamten Forschungsausgaben auf Grundlagenforschung und 78\% auf angewandte Forschung.

Die Universitäten und Zentren der Grundlagenforschung erbringen auf zwei Wegen wichtige Vorleistungen für die private Innovation und forschungsnahe Lehre. Die Ausbildung an der Grenze der Forschung stellt der Privatwirtschaft hochqualifiziertes Forschungspersonal bereit. Die Ergebnisse der Grundlagenforschung sind allgemein verfügbar und werden in der Privatwirtschaft $\mathrm{zu}$ kommerziell verwertbaren neuen Anwendungen und Produkten weiterentwickelt. Der Zugang zu Forschungspersonal und zu neuen Entwicklungen der Forschung ist für F\&E-intensive Unternehmen ein wichtiges Argument für die Standortentscheidung. Gerade eine hochentwickelte Wirtschaft, die an die technologische Grenze vorstößt, muss mehr in Grundlagenforschung investieren als Länder, die mit einer Nischenstrategie und kleinen hochspezialisierten Verbesserungen erst aufschließen. Mit Innovation in kleinen Schritten können die vielen Hidden Champions auch nicht zu ganz großen Konzernen heranwachsen. Die führenden Innovationsländer müssen die Grenze der Forschung selbst hinausschieben und brauchen radikale Innovationen, um auch völlig neue Märkte und Anwendungen zu erschließen und dort eine weltweit dominierende Stellung zu besetzen. Abbildung 27 zeigt im internationalen Vergleich, wie die Ausgaben für Grundlagenforschung steigen, wenn die Länder reicher werden und sich der technologischen Grenze annähern. Die Nähe zur technologischen Grenze wird dabei mit dem Abstand des BIP pro Kopf zu jenem der USA als führendes Innovationsland gemessen.

Die Ausgaben für Grundlagenforschung nützen insbesondere der Industrie und unterstützen die Spezialisierung der Wirtschaft auf den Hochtechnologiebereich. Zu Beginn des Produktzyklus besteht noch eine enge Synergie zwischen Produktion und F\&E, die daher am besten zusammen innerhalb des Unternehmens verbleiben. So können die Erfahrungen in der Produktion und im Verkauf am ehesten und schnellsten in die Weiterentwicklung des Produkts eingehen. Wenn das Produkt zunehmend ausgereift ist und ähnliche Angebote der Konkurrenz auf den Markt kommen, können die Unternehmen standardisierte Tätigkeiten 
und die Produktion mit genau spezifizierten Qualitätsmerkmalen leichter in lohngünstigere Länder auslagern, um Kosten zu senken und die Wettbewerbsfähigkeit zu erhalten (vgl. z.B. Antras, 2005). Produktion und F\&E können leichter getrennt werden. Das bedeutet, dass die Unternehmen sich auf die forschungsintensivsten Teile der Wertschöpfungskette spezialisieren, die der eigentlichen Produktion vor- und nachgelagert sind. Dazu zählen F\&E, Design, Markenbildung und ähnliches, während die standardisierte Produktion an unabhängigere und kostengünstigere Unternehmen vergeben wird.

Abbildung 27: Grundlagenforschung und wirtschaftliche Entwicklung
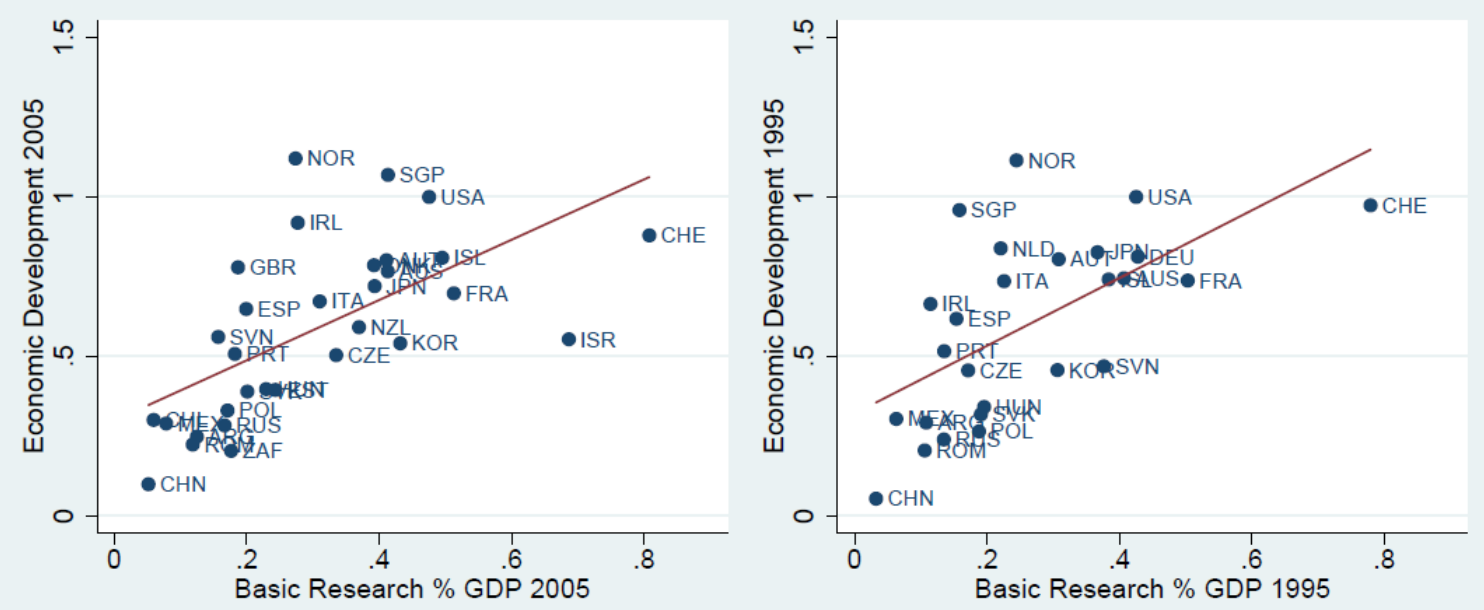

Quelle: Gersbach u.a. (2015, Figure 3) nach Daten der OECD und Weltbank; horizontal: Ausgaben Grundlagenforschung in \% des BIP 1995, vertikal: wirtschaftliche Entwicklung 2005 gemessen am Verhältnis BIP pro Kopf/U.S. BIP pro Kopf, in Kaufkraftparitäten

Bernard u.a. (2016) erklären damit einen guten Teil des Rückgangs der industriellen Beschäftigung in Dänemark. Seit Mitte der 1980er-Jahre sank die Zahl der produzierenden Firmen netto (Neugründungen minus Betriebsaufgaben) um etwa 1,4\% jährlich. Firmen können komplett schließen oder ihr Geschäft in den nicht produzierenden Sektor verlagern, indem sie sich auf F\&E, Design und Engineering, Markenbildung und Verkauf spezialisieren. Im Durchschnitt wechselten etwa $1,6 \%$ der produzierenden Firmen in den Dienstleistungssektor, umgekehrt sind es 1,1\% jährlich. Die 1.316 Firmen, die zwischen 2002 und 2007 in den nicht-produzierenden Sektor abwanderten, haben fast 10\% der aktiven, produzierenden Firmen im Jahr 2002 ausgemacht und verursachten $42 \%$ der Arbeitsplatzverluste seitdem. Während die Beschäftigung in der Industrie deutlich sank, stieg sie in den neu orientierten Unternehmen um 17\%. Nach der Neuorientierung haben viele dieser Firmen die hochqualifizierte Beschäftigung stark ausgebaut und gehörten am Ende zu den F\&E-intensivsten Unternehmen überhaupt. Weil sie nach der Restrukturierung kaum noch produzieren, werden sie als Teil des Dienstleistungssektors gezählt, obwohl sie ähnliche „produktionsnahe“ Tätigkeiten ausüben wie vorher. Der enge Fokus auf die Industrie wirkt daher in der Debatte um De- und Re-Industrialisierung etwas künstlich.

Die Grundlagenforschung ist eine Vorleistung für die private Forschung. Universitäten liefern Wissen (Patente) und F\&E-Personal. Wie stark können Mehrausgaben für die Grundlagenforschung die private Forschung anregen? Jaffe (1989) quantifizierte für die USA 
den kausalen Effekt der Grundlagenforschung auf die industrielle Innovation. Demnach steigen im Durchschnitt der betrachteten Branchen die privaten F\&E-Ausgaben um 7\%, wenn die Ausgaben für Grundlagenforschung um 10\% wachsen. Eine Zunahme der industriellen Wertschöpfung hat in etwa einen gleich großen Effekt. Auch die privaten Patentanmeldungen nehmen mit der universitären Forschung zu, und zwar mit einer Elastizität von 0,1. Steigen die Ausgaben für Grundlagenforschung um 10\%, nehmen die Patentanmeldungen in der Industrie um 1\% zu. Da die universitäre Forschung nur etwa ein Sechstel der privaten F\&EAusgaben ausmacht, ist das ein bedeutender Effekt. Die universitäre Forschung trägt aber auch indirekt zur Patenthäufigkeit bei, weil die Grundlagenforschung mehr private F\&E auslöst (Elastizität 0,7) und diese wiederum die Zahl der Patente (Elastizität 0,8) steigert. Die totale Elastizität beträgt daher 0,6. Investiert der Staat $10 \% \mathrm{mehr}$ in die Grundlagenforschung, nehmen die industriellen Patente um 6\% zu. Toole (2012) findet ähnliche Resultate speziell für die pharmazeutische Industrie. Er bildete aus dem Strom von Projekten einen kumulativen Wissensbestand der öffentlichen Grundlagenforschung. Ein Zuwachs um $1 \%$ steigert demnach den privaten F\&E-Output neuer Medikamente und Therapien um 1,8\%. Allerdings wirken sich die staatlichen Investitionen nur sehr langsam mit einer Verzögerung von 17 bis 24 Jahren aus. Zudem berechnete der Forscher eine Rendite von $43 \%$ auf die staatlichen Investitionen in die pharmazeutische Grundlagenforschung.

Die Wirkungen auf private Innovation ergeben sich jedoch nicht rein mechanisch und können durch spezielle Initiativen des Technologietransfers verstärkt werden. In Norwegen hatten universitäre ForscherInnen umfassende Rechte an den von ihnen geschaffenen Innovationen und insbesondere an den Einkünften aus Start-ups und Patenten. Hvide und Jones (2016) untersuchen, wie sich die Abschaffung dieser Anreize im Jahr 2003 auswirkte. Von den 48.844 Start-ups, die im Zeitraum zwischen 2000 und 2007 in Norwegen gegründet wurden, stammten 128 aus den Universitäten. Zwischen 1995 und 2010 wurden zudem 750 Patentanmeldungen durch 431 universitäre Forschende gezählt. In ganz Norwegen haben 6.890 ErfinderInnen total 7.341 Patente angemeldet. Während die Zahl der nichtuniversitären Start-Ups unverändert blieb, ist mit der Abschaffung des „Professorenprivilegs“ ein starker Rückgang von 56\% bei universitären Neugründungen auszumachen. Sie fielen von 24,7 vor der Reform (2000-2002) auf jährlich 10,8 Start-ups danach (2003-2007). Die Autoren schätzen, dass die Abschaffung der Anreize die Anzahl der Start-ups von universitären ForscherInnen (PhD-AbsolventInnen) zwischen 49 und 67\% reduzierte. Nicht der Weggang der ForscherInnen ist für diesen Rückgang verantwortlich, sondern die geringere Neigung, Zeit und Aufwand in die Kommerzialisierung zu stecken. Zudem scheint die Reform auch die Qualität der Neugründungen beeinträchtigt zu haben. Die Überlebenswahrscheinlichkeit universitärer Start-ups sank um 15 Prozentpunkte.

Ähnliche Ergebnisse stellen Hvide und Jones (2016) auch bei der Zahl der universitären Patente fest. Die Patentanmeldungen einer Universität gingen um 20\% und jene einer universitären Forscherin bzw. eines universitären Forschers um 48\% zurück. Die Abschaffung der Professorenbeteiligung reduziert die Wahrscheinlichkeit, dass eine akademische Forscherin bzw. ein akademischer Forscher ein Patent entwickelt, um 4,5 Prozentpunkte. Die Autoren konnten zeigen, dass der Grund für diesen Einbruch nicht im Weggang von besonders aktiven Forschenden, sondern vielmehr darin liegt, dass sie nach der Reform wesentlich seltener Patente entwickelten. Die ungünstige Anreizwirkung der Reform 
mindert auch die Qualität der universitären Patente, gemessen an der Anzahl ihrer Zitationen. Die Häufigkeit, dass ein Patent genannt wird, ging um rund 25\% zurück. Auch an den Universitäten bringt also eine Schwächung der Eigentumsrechte eine starke Abnahme der davon betroffenen Forschungsaktivitäten mit sich. Wer mehr Technologietransfer haben will, muss das Interesse der in Frage kommenden ForscherInnen wecken, kommerzialisierbare Anwendungen zu erschließen, und sie am Erfolg dieser Aktivitäten beteiligen.

Universitäten und multinationale Konzerne konkurrieren weltweit um führende ForscherInnen, die überdurchschnittlich mobil sind. Akcigit u.a. (2015) haben die Mobilität von besonders leistungsfähigen ForscherInnen und ErfinderInnen mit überaus hohem Einkommen in Abhängigkeit von den Spitzensteuersätzen der Einkommensteuer untersucht. Sie konzentrieren sich dabei auf „StarforscherInnen“, die bei besonders vielen Patenten eine wichtige Rolle spielen und überaus oft zitiert werden. Der Anteil der im Inland verbleibenden StarforscherInnen hat eine Elastizität von 0,165. Wenn also das Nettoeinkommen (nach einer Steuersenkung) um 10 Prozent steigt, nimmt der Anteil der im Inland bleibenden ForscherInnen um 1,65 Prozent zu. Dieser Zusammenhang gilt für das oberste Einkommensviertel und ist für ForscherInnen mit geringerem Einkommen nicht mehr signifikant. Die Besteuerung hat also einen Einfluss auf die Wahrscheinlichkeit, dass sich eine Erfinderin bzw. ein Erfinder oder überhaupt Forschende in einem Land ansiedeln oder dort verbleiben. Dabei reagieren einheimische und ausländische Personen unterschiedlich stark. Die Elastizität der einheimischen ErfinderInnen bezüglich des Einkommens nach Steuern beträgt 0,04, wohingegen ausländische ForscherInnen mit einer Elastizität von 1,29 deutlich sensibler reagieren. Bei einer Absenkung des Spitzensteuersatzes um 10\% würden also die USA um 0,1\% mehr von den einheimischen ErfinderInnen halten können, aber auch um 23,7\% mehr ausländische ErfinderInnen anziehen können.

\subsection{Fiskalische F\&E-Anreize}

\subsubsection{Alternative Instrumente}

Grundlagenforschung und Patentschutz ändern nichts daran, dass ein Teil der privaten F\&EErträge auf andere Firmen übergeht, ohne dass dafür ein Preis verrechnet werden könnte. Da die Firmen diese externen Erträge nicht bezahlt bekommen, investieren sie zu wenig in die Forschung. Die Gesamtwirtschaft schöpft ihr Potenzial für Wachstum und Wohlfahrt nicht aus. Um die Innovationsanreize zu stärken, kann der Staat den Verursachern ihre externen Erträge entweder mit Direktzahlungen oder mit Steuernachlässen ersetzen. Diesem Zweck dienen die direkte Forschungsförderung, spezielle F\&E-Steuerabzüge und eine begünstigte Besteuerung von Innovationserträgen im Rahmen von Patentboxen. Wie bedeutend die externen Erträge sind, und wie umfangreich die Förderung daher sein sollte, ist quantitativ schwierig festzumachen. Der internationale Vergleich in Abbildung 28 zeigt, dass die Staaten in sehr unterschiedlichem Ausmaß F\&E-Förderung betreiben. 
Abbildung 28: Steuerliche Subventionsrate der F\&E-Ausgaben, 2015

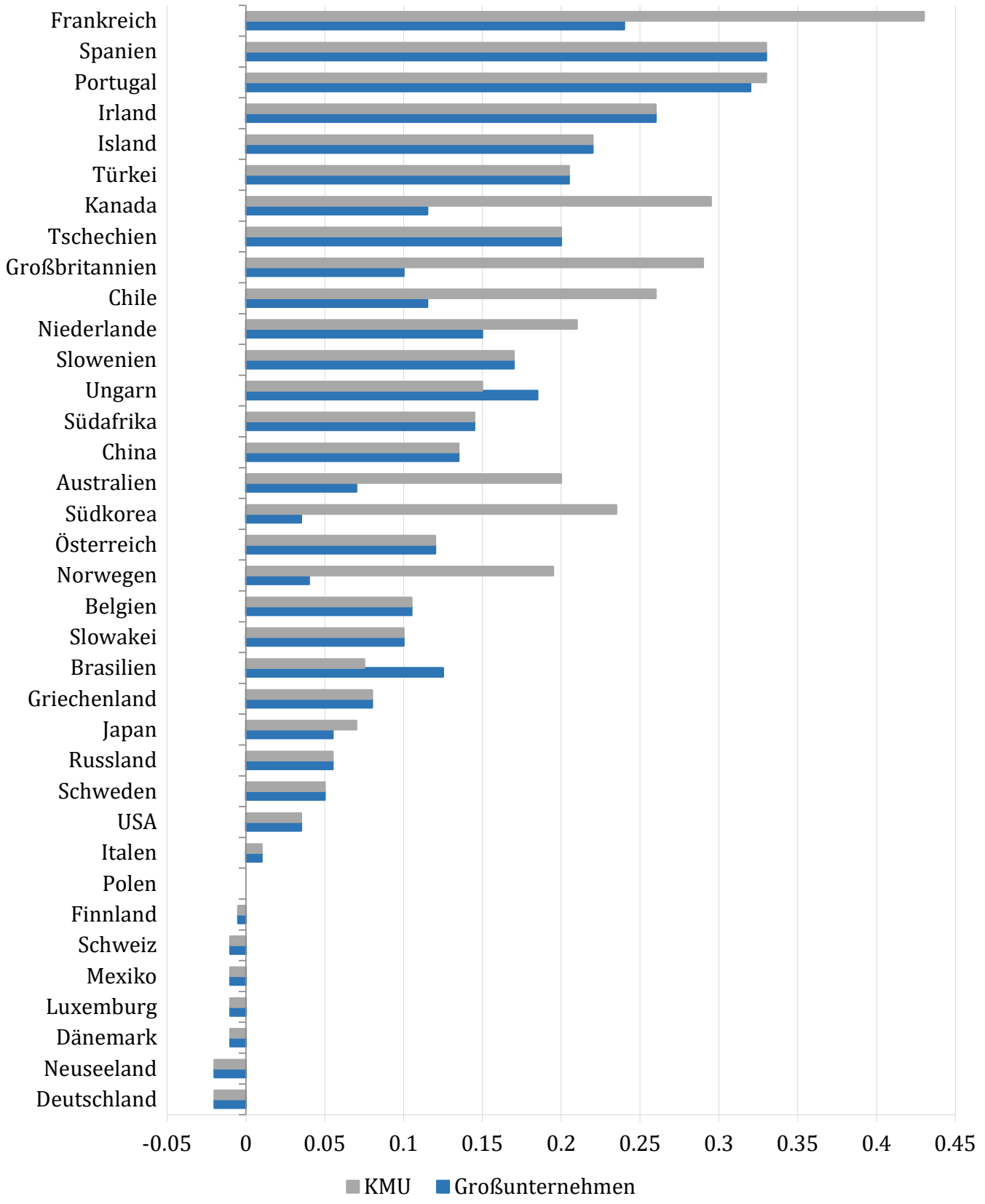

Quelle: OECD (http://dx.doi.org/10.1787/888933274335), eigene Darstellung

Die Begünstigung von Patenterträgen und ein spezieller F\&E-Steuerabzug sind tendenziell gleichwertig, wenn sie dieselbe Firma betreffen. Sie unterscheiden sich lediglich darin, dass die Entlastung beim Steuerabzug sofort eintritt, während sie bei den Patenterträgen erst später anfällt. Die Sätze können so gewählt werden, dass im Barwert dieselbe Entlastung erzielt wird. Die Steuerbegünstigung tritt automatisch ein, wenn die Firmen sie beantragen. Ein administrativer Aufwand entsteht allenfalls in Abgrenzungsfragen, was genau als F\&EAusgaben gelten kann, wenn Firmen allzu großzügig solche Abzüge beanspruchen. 
Die F\&E-Subventionen werden dagegen durch spezielle Fördergesellschaften vergeben. Sie fallen nicht automatisch an, sondern müssen beantragt werden und unterliegen einem erheblichen Risiko der Nicht-Genehmigung. Die Behörden müssen die Förderungswürdigkeit beurteilen und dann ein knappes Budget auf rivalisierende Anträge aufteilen. Ein Vorteil ist, dass knappe Steuergelder sparsam und gezielt für die Projekte mit dem größten Potenzial reserviert bleiben. Andererseits ist es fraglich, ob eine öffentliche Behörde das kommerzielle Potenzial von F\&E-Ausgaben besser als der Markt beurteilen kann. Auch die Förderinstitutionen müssen die Wirkung ihres Budgets rechtfertigen und haben daher einen Anreiz, die Gelder eher für sichere Projekte einzusetzen und an Firmen zu vergeben, die schon erfolgreich sind. Die radikalsten und riskantesten Innovationen, die am dringendsten Finanzierung brauchen, könnten zu kurz geraten, während erfolgreiche Unternehmen eine Förderung nicht brauchen und die Projekte auch sonst finanziert hätten. Dass mit dem Antragsverfahren größere Vorbereitungskosten verbunden sind, benachteiligt kleine KMU, weil sie die gleichen Fixkosten auf einen wesentlich kleineren Umsatz verteilen müssen.

Die eingesetzten Steuermittel haben viele dringende alternative Verwendungen. Auch das Budget für F\&E-Förderung muss sich daher mit seinem volkswirtschaftlichen Mehrwert rechtfertigen. Der Unterschied zwischen der sozialen und privaten Rendite von F\&E ist der volkswirtschaftliche Mehrwert, den eine F\&E-Investition erzielt. Die F\&E-Förderung rechnet sich für den Staat und die Gesellschaft nur, wenn genügend viele zusätzliche F\&EInvestitionen entstehen, deren Mehrwert das F\&E-Förderbudget übersteigt. Auch die F\&EFörderung muss den Test einer Kosten-Nutzen-Analyse bestehen. Die entscheidende Frage ist daher: Wie stark kann die F\&E-Förderung tatsächlich die privaten F\&E-Ausgaben der Wirtschaft steigern? Dazu gibt es eine umfangreiche empirische Evidenz.

\subsubsection{Spezielle F\&E-Steuerabzüge}

Wie Abbildung 28 zeigt, engagieren sich viele Staaten teilweise sehr stark in steuerlicher F\&EFörderung und differenzieren dabei teilweise zwischen großen und kleinen Unternehmen. Für die Zwecke dieser Studie ist die neue und einflussreiche Forschungsarbeit von Dechezlepretre u.a. (2016) zur steuerlichen F\&E-Förderung in Großbritannien (GB) besonders interessant. Seit 2000 können KMUs in GB 150\% der F\&E-Ausgaben für Personalund Sachaufwand von der Steuer absetzen, und große Unternehmen 125\%. Im Jahr 2008 erfolgte eine Reform. Sie weitete den Kreis der begünstigten KMUs aus, indem sie die maximalen Vermögenswerte, um als KMU zu gelten, von 43 auf 86 Mio. Euro anhob. Zudem hat die Regierung die F\&E-Steuerabzüge für KMU von 150 auf $175 \%$ und für große Unternehmen von 125 auf 130\% angehoben. Konkret untersuchen die WissenschaftlerInnen Daten von 5.888 britischen Unternehmen für den Zeitraum von 2006 bis 2011. Die Firmen verfügten vor der Reform 2008 über Aktiva von 61 bis 111 Mio. Euro. Jene, deren Vermögenswerte unter den Schwellenwert von 86 Mio. Euro fallen, wurden durch die Reform neu als KMU klassifiziert und kamen ab 2008 in den Genuss der höheren Steueranreize.

Vor der Reform beliefen sich die durchschnittlichen F\&E-Ausgaben eines Unternehmens auf $£ 72.300$. Die Reform hat die Ausgaben um $£ 75.300$ gesteigert und somit mehr als verdoppelt! Die Reform hatte den größten Effekt dort, wo bereits vorher hohe F\&E-Investitionen getätigt wurden. Ähnliches zeigt sich bei Patentanmeldungen. Vor der Reform meldete ein durchschnittliches Unternehmen 0,06 Patente pro Jahr an, nachher kamen 0,035 hinzu, das 
entspricht einer Steigerung um 58\%. Ein möglicher Einwand ist, dass die Steuerreform zu mehr, aber qualitativ schlechteren Innovationen geführt haben könnte. Die AutorInnenen zeigen, dass ein solcher Nebeneffekt unwahrscheinlich ist. Ein beim Europäischen Patentamt registriertes Patent ist sechsmal so teuer wie ein britisches Patent, aber auch wesentlich wertvoller, weil es in den allermeisten Ländern der EU gleichzeitig registriert und geschützt ist und daher eine höhere Qualität hat. Die Untersuchung zeigt, dass beide Patenttypen mit dem gleichen Faktor 1,2 zugenommen haben. Es gibt also kaum Anzeichen für Mitnahmeeffekte, und die Innovationsqualität ist eher gestiegen.

Steuervergünstigungen senken die privaten F\&E-Kosten. Die ForscherInnen schätzen eine Steuerpreiselastizität von 2,6. Demnach steigen die F\&E-Investitionen um 2,6\%, wenn die Kosten nach Einrechnung des Steuervorteils um 1\% sinken. Andere Studien kommen typischerweise auf Werte zwischen eins und zwei. Dieser Unterschied dürfte damit zu erklären sein, dass der Datensatz zahlreiche kleinere Firmen enthält. Diese haben es schwerer, an Kredite zu kommen und ihre F\&E-Aktivitäten zu finanzieren. Deshalb reagieren sie stärker auf Steuererleichterungen und steigern die F\&E-Aktivitäten relativ mehr. Um diesen Unterschied herauszuarbeiten, spalten die WissenschaftlerInnen die Stichprobe nach Firmenalter auf und schätzen bei jüngeren Firmen mit 4,7 eine wesentlich höhere Steuerpreiselastizität als bei älteren mit 1,6. Schließlich berechnen die ForscherInnen, wie viel zusätzliche F\&E der Einsatz eines britischen Pfunds an Steuergeldern auslöst. Sie stellen den durchschnittlichen Zuwachs von F\&E-Ausgaben von £1,64 Mrd. pro Jahr den entgangenen Steuereinnahmen von $£ 0,96$ Mrd. gegenüber. Das ergibt ein Preis-LeistungsVerhältnis von rund 1,7 und bedeutet, dass eine Steuerentlastung von $£ 1$ rund $£ 1,7$ an neuer F\&E ausgelöst hat. Die F\&E-Ausgaben wären ohne Reform deutlich niedriger gewesen.

Agrawal u.a. (2014) untersuchen eine Anpassung der Qualifikationsregeln für F\&ESteuerguthaben in Kanada, um die Wirksamkeit fiskalischer F\&E-Anreize für kleine Firmen zu ermitteln. In Kanada können alle Unternehmen 120\% ihrer inländischen F\&E-Ausgaben vom steuerbaren Gewinn abziehen, wobei die zusätzlichen $20 \%$ eine nicht rückzahlungspflichtige Steuergutschrift sind. KMU können weitere 15\%, also insgesamt 135\%, der F\&E-Ausgaben als Steuergutschrift geltend machen. Allerdings ist diese zusätzliche Steuergutschrift rückzahlungspflichtig und steht den Firmen nur bis zu einer Ausgaben-Höchstgrenze zu. Vor der Reform im Jahr 2004 konnten Firmen mit einem Vorjahresgewinn von bis zu 200.000 CND die ersten zwei Mio. an F\&E-Ausgaben zu 135\% absetzen. Der 15\%ige KMU-Bonus wurde mit steigenden Gewinnen auf Null abgeschmolzen. $\mathrm{Ab}$ einem Gewinn von 400.000 CND bestand nur mehr ein Anspruch auf die normale Gutschrift für 20\% (d.h. es waren nur mehr 120\% der F\&E-Ausgaben absetzbar), während der KMU-Bonus vollständig entfiel. Nach der Reform wurde die Anspruchsberechtigung auf den KMU-Bonus für die ersten 2 Mio. an F\&E-Ausgaben ausgeweitet und erst ab einem höheren Vorjahresgewinn zwischen 300.000 bis 500.000 CND wieder zurückgenommen. Kurzum, die Reform weitete die Anspruchsberechtigung auf den KMU-Bonus aus, so dass mehr Firmen von geringeren Nach-Steuer-Kosten für F\&E-Ausgaben profitieren konnten.

Die ForscherInnen haben die Steuerunterlagen von Firmen untersucht, die von 2000 bis 2007 von den Steueranreizen profitiert haben. Der Datensatz umfasste 7.239 Firmen mit 48.638 Beobachtungen. Von diesen Firmen waren 50\% im Dienstleistungssektor tätig, 29\% in der verarbeitenden Industrie und die restlichen $21 \%$ in anderen Sektoren, vornehmlich 
Landwirtschaft. Die durchschnittlichen F\&E-Ausgaben betrugen 82.887 CND pro Jahr, wovon $66 \%$ für Löhne, 17\% für ausgelagerte Forschung und 3,6\% für Kapital ausgegeben wurden. Die Schätzungen zeigen zum einen, dass Firmen, die sich für die erwähnten Steuergutschriften qualifizierten, höhere F\&E-Ausgaben tätigen als nicht anspruchsberechtigte Firmen. Bei Firmen, die nach der Reform sich erstmals für den KMUBonus qualifizieren konnten, hat die Reduktion der F\&E-Kosten im Durchschnitt um 17\% höhere F\&E-Ausgaben ausgelöst.

Um die Ergebnisse mit anderen Studien zu vergleichen, berechnen die ForscherInnen die Elastizität der F\&E-Ausgaben bezüglich der steuerlich korrigierten F\&E-Grenzkosten und erhalten einen Wert von -1,5. Wenn die privaten Grenzkosten für F\&E um 1\% sinken, steigen die F\&E-Ausgaben um 1,5\%. Dabei reagieren die Komponenten der F\&E-Ausgaben unterschiedlich stark. Während die F\&E-Ausgaben für externe AuftragnehmerInnen mit -3 überdurchschnittlich stark reagieren, ist die Kostenelastizität bezüglich F\&E-Löhnen mit 1,05 deutlich kleiner. Es ist eben kostspieliger und schwieriger, den eigenen F\&EPersonalbestand entsprechend stark anzupassen. Die durchschnittliche Elastizität von -1,5 ist etwas höher als die traditionellen Schätzungen, die z.B. nach dem Literaturüberblick von Becker (2015) um Werte von -1 streuen. Neuere Forschungsarbeiten wie die vorhin besprochene Arbeit von Dechezlepretre (2016) kommen jedoch auf durchaus höhere Werte, vor allem für kleine Firmen, die häufig mit einem beschränkten Zugang zu externer Finanzierung zu kämpfen haben.

Viele Länder in der EU geben Steueranreize für F\&E. Österreich gewährt seit 2002 eine Forschungsprämie, die schrittweise von $3 \%$ in 2002 auf 5\% in 2003, 8\% ab 2004 und 10\% ab 2011 auf zuletzt 12\% ab 1.1.2016 ausgebaut wurde (\$108c EStG). Bis 2010 konnte der Forschungsfreibetrag beansprucht werden, der danach wegfiel. Während es für die eigenbetriebliche Forschung keine Höchstgrenze gibt, ist die Forschungsprämie bei Auftragsforschung nur für Aufwendungen bis max. 1 Mio. Euro pro Jahr zulässig. Die EUKommission schlägt im Rahmen eines Vorstoßes für eine gemeinsame konsolidierte Bemessungsgrundlage der Körperschaftssteuer (ССТВ, common consolidated corporate tax base) einen gestaffelten Steuerabzug vor, wonach große Unternehmen auf die ersten 20 Mio. Euro an F\&E-Ausgaben 150\% und auf darüberhinausgehende Aufwendungen 125\% steuerlich absetzen können. Ein F\&E-Aufwand von 30 Mio. würde demnach die Bemessungsgrundlage um 42,5 Mio. Euro mindern. Kleine Unternehmen sollen nach diesem Steuerplan 200\% der F\&E-Ausgaben absetzen können. Der Steuerplan schlägt zudem vor, Eigenkapital- und Fremdkapitalfinanzierung steuerlich gleich zu behandeln, und will damit die Finanzierung mit Risikokapital auf dem Kapitalmarkt der Kreditfinanzierung über Banken steuerlich gleich behandeln (vgl. dazu Abschnitt 4.4.5).

\subsubsection{Begünstigung von F\&E-Erträgen in Patentboxen}

Marken, Patente, Urheberrechte und Prozessinnovationen leisten immer stärkere Beiträge zur Wertschöpfung und sind international mobil. Global tätige Unternehmen können die Erträge von immateriellen Wirtschaftsgütern sehr leicht verlagern, indem sie z.B. Patente in anderen Ländern anmelden und dort die Gewinne versteuern, die aus den Nutzungsgebühren der Tochterfirmen weltweit stammen. Um die Bemessungsgrundlage zu schützen und die Ergiebigkeit der Steuer zu erhalten, sind viele Länder dazu übergegangen, im Rahmen von 
Patentboxen F\&E-Erträge (Lizenzerträge aus Patenten, Markenrechten und anderen Formen des geistigen Eigentums) steuerlich zu entlasten.

Tabelle 13: Patentboxen in Europa, Stand 2014

\begin{tabular}{|c|c|c|c|c|}
\hline Land & $\begin{array}{l}\text { Steuersatz } \\
\text { IP-Box, \% }\end{array}$ & $\begin{array}{l}\text { KÖSt-Satz, } \\
\text { normal, \% }\end{array}$ & IP-Formen & $\begin{array}{l}\text { Ein- } \\
\text { führung }\end{array}$ \\
\hline Belgien & 6,8 & 33,99 & u.a. Patente und SPC & 2007 \\
\hline Zypern & 2,5 & 12,5 & $\begin{array}{l}\text { u.a. Patente, Designs, Muster, } \\
\text { Markennamen und Kundenlisten }\end{array}$ & 2012 \\
\hline Frankreich & 16,76 & 35,41 & $\begin{array}{l}\text { u.a. Patente, SPC und Produktions- } \\
\text { prozesse auf Basis von Patenten }\end{array}$ & 2000 \\
\hline Ungarn & 9,5 & 19 & $\begin{array}{l}\text { u.a. Patente, Designs, Muster, } \\
\text { Markennamen und Kundenlisten }\end{array}$ & 2003 \\
\hline Liechtenstein & 2,5 & 12,5 & $\begin{array}{l}\text { u.a. Patente, Copyrights (inkl. } \\
\text { Software), Designs und Muster }\end{array}$ & 2011 \\
\hline Luxemburg & 5,84 & 29,22 & $\begin{array}{l}\text { u.a. Patente, SPC, Designs, } \\
\text { Muster und Markennamen }\end{array}$ & 2008 \\
\hline Malta & 0 & 35 & $\begin{array}{l}\text { Patente, Markenrechte, } \\
\text { Urheberrechte }\end{array}$ & 2010 \\
\hline Niederlande & 5 & 25 & $\begin{array}{l}\text { Patente und IP, für die } \\
\text { F\&E-Zertifikate ausgestellt sind }\end{array}$ & 2007 \\
\hline Portugal & 15 & 30 & Patente, Lizenzen, Designs und Modelle & 2014 \\
\hline Spanien & 12 & 30 & $\begin{array}{l}\text { Patente, Pläne, Muster, } \\
\text { Geheimformeln und Prozeduren }\end{array}$ & 2008 \\
\hline UK & 10 & 21 & $\begin{array}{l}\text { Patente, SPC und mit Patenten } \\
\text { vergleichbare Schutzrechte }\end{array}$ & 2013 \\
\hline $\begin{array}{l}\text { Kanton } \\
\text { Nidwalden }\end{array}$ & 8,8 & 12,66 & $\begin{array}{l}\text { u.a. Patente, Geheimformeln, } \\
\text { Prozesse, Know-how, Markenrechte }\end{array}$ & 2011 \\
\hline
\end{tabular}

Quelle: Evers u.a. (2015)

Tabelle 13 stellt nach Evers u.a. (2015) die seit dem Jahr 2000 von zehn EU-Mitgliedstaaten und dem Schweizer Kanton Nidwalden gewählten Lösungen für IP-Boxen (Intellectual Property) zusammen. Oft betragen die reduzierten Sätze deutlich weniger als die Hälfte der normalen Gewinnsteuersätze. Die ForscherInnen kommen zum Schluss, dass IP-Boxen eher für kleinere Länder vorteilhaft sind, weil die Steuerausfälle aus der Gewährung des verringerten Steuersatzes durch positive Effekte aus Ansiedlungen und zusätzlichen Investitionen in IP überkompensiert werden. Bei größeren Staaten überwiegen dagegen die Steuerausfälle.

Prinzipiell kann eine Regierung mit einer begünstigten Besteuerung von Innovationserträgen eine gleich hohe Förderung erzielen wie mit zusätzlichen Steuerabzügen für F\&E-Ausgaben. Allerdings ist der Kreis der begünstigten Unternehmen bei Patentboxen meist enger gezogen, weil viele kleine KMU wegen des hohen Aufwands keine Patente anmelden oder keine ähnlich qualifizierenden Innovationserträge vorweisen können. Zudem können Patentboxen oft eine 
besonders aggressive Strategie im Steuerwettbewerb sein, wenn der Zusammenhang zu den vorher getätigten Forschungsaufwendungen fehlt. Wenn z.B. ein multinationales Unternehmen in Großbritannien ein Patent entwickelt, aber das Patent dann in Belgien ansiedelt und dort die Lizenzerträge versteuert, sind die Steuerersparnisse gewaltig. Der KÖSt-Satz in GB beträgt 21\%, wobei 125\% der F\&E-Ausgaben abzugsfähig sind, während in Belgien die Lizenzgebühren in der Patentbox nur mit 6,8\% (bei einem Normalsteuersatz von fast $40 \%$ ) besteuert werden. So kann Belgien seine Steuerbasis auf Kosten von GB deutlich steigern, ohne dass vorher die F\&E-Ausgaben zu Steuerausfällen geführt hätten. Um den Steuerwettbewerb fair zu gestalten und zu verhindern, dass Länder nur um die Steuerbasis losgelöst von der zugrundeliegenden wirtschaftlichen Aktivität konkurrieren, besteht die OECD im Rahmen der BEPS-Initiative (Base Erosion and Profit Shifting) auf das KonnexitätsPrinzip. Die Patenterträge sollen nur dann für eine Begünstigung in einer Patentbox qualifizieren, wenn auch die zugrundeliegenden F\&E-Ausgaben im selben Land stattgefunden und die nationale Bemessungsgrundlage vorher reduziert haben.

Die empirische Forschung bestätigt die Wirksamkeit von Patentboxen. Karkinsky und Riedl (2012) untersuchen einen Datensatz mit über 60.000 Beobachtungen zwischen 1995 und 2003 von 9.145 europäischen Gesellschaften, die zu multinationalen Unternehmen gehören. Pro Jahr meldet eine Gesellschaft durchschnittlich 0,9 Patente beim Europäischen Patentamt an. Der durchschnittliche Steuersatz beträgt 39\% und variiert je nach Land zwischen 10 und 59\%. Die Erhöhung des Steuersatzes um zehn Prozentpunkte reduziert im selben Land die Zahl der Anmeldungen um 35\%. Die Patentanmeldungen gehen auch dann stark zurück, wenn zwar der eigene Steuersatz unverändert bleibt, aber andere Standortländer ihre Steuersätze senken. Der Anreiz steigt, die Patente in den Ländern mit tieferer Steuerbelastung anzumelden. Eine Patentbox reduziert selektiv den Steuersatz auf Patenterlöse, steigert daher die Patentanmeldungen und zieht Patente aus anderen Ländern an.

Ziel von Patentboxen ist es, Innovationen zu fördern und die eigene Steuerbasis zu schützen. Zunächst weisen Griffith u.a. (2014) darauf hin, dass die Einführung von Patentboxen nach der bisherigen Praxis (Untersuchungszeitraum von 1985 bis 2005) zu einer starken Verlagerung der Patentanmeldungen und damit zu einer erheblichen internationalen Umverteilung von Steuereinnahmen geführt hat. Die Innovationstätigkeit im Land mit einer Patentbox muss deshalb nicht unbedingt zunehmen. Die Firmen tätigen F\&E-Investitionen in einem Land und siedeln die Patente in anderen, steuergünstigeren Ländern an, ohne dass eine Verlagerung der F\&E-Einheiten notwendig wäre. Staaten mit Patentboxen konnten ihre Steuereinnahmen auf Kosten anderer steigern. Da Multis mit der Verschiebung von Patenten ihre weltweite Steuerschuld reduzieren, müssen zwangsläufig die totalen Steuereinnahmen aller Länder abnehmen. Bradley u.a. (2015) zeigen dagegen, dass Patentboxen sehr wohl die Innovation steigern, ohne dass es zu einer signifikanten Verlagerung der Patente käme. Eine Absenkung des begünstigten Steuersatzes in der Patentbox um einen Prozentpunkt löst demnach eine Zunahme neuer Patentanträge um 3\% aus.

Diesen augenscheinlichen Widerspruch lösen Alstadsaeter u.a. (2015) auf. In ihrem Datensatz beträgt die durchschnittliche Begünstigung der Unternehmenssteuer mit einer Patentbox ca. 18\%. Die Einführung des Nexus-Ansatzes würde in der Automobilindustrie die Wahrscheinlichkeit um 60\% steigern, dass F\&E-Aktivitäten und Patentverwertung am selben Ort stattfinden. In der Chemischen Industrie bzw. in der Informations- und Computerbranche 
nähmen dieselben Wahrscheinlichkeiten um 45\% bzw. 30\% zu. Der Nexus-Ansatz kann den Anreiz zur Patentverlagerung eindämmen, so dass eine fiskalische Förderung via Patentboxen und F\&E-Steuerabzüge, d.h. eine Begünstigung der Erträge und eine Subventionierung der Kosten, ähnliche Effekte haben sollte, zumindest was die Quantität von F\&E-Aktivitäten betrifft. Ernst u.a. (2014) zeigen allerdings, dass zwar die quantitativen Effekte der beiden Instrumente ähnlich sind, aber Patentboxen im Unterschied zu F\&E-Steuerabzügen deutlich die Qualität der Innovationen steigern. Während die Steuerabzüge alle F\&E-Investitionen begünstigen, kommt der Steuervorteil in einer Patentbox überproportional den innovativeren Projekten mit hoher Profitabilität zugute. Sie messen Innovationsgrad und Profitabilität der Patente mit der Anzahl der Patentzitationen, der Größe der Patentfamilie (Anzahl der Länder, in denen das Patent geschützt ist) und der Zahl der im Patent genannten Industrieklassen. Der Effekt ist ökonomisch relevant: Ein um 10 Prozentpunkte niedrigerer Steuersatz auf Patenterträge steigert die Patentqualität um 1 bis 5\%.

\subsubsection{Direkte Subventionen}

F\&E-Subventionen sind direkte Projektbeiträge, die der Höhe nach als fixer Betrag oder als variabler, anteilsmäßiger Zuschuss fließen können. Ein fixer Betrag hat keinen direkten Einfluss auf die marginalen F\&E-Kosten, so dass im Normalfall kein Anreiz für eine Ausweitung des Umfangs der F\&E-Investition entsteht (intensiver Effekt). Eine positive Wirkung sollte sich jedoch bei finanzierungsbeschränkten Unternehmen einstellen, da jede Subvention die Finanzierungskapazität steigert und damit eine Ausweitung der F\&EAusgaben ermöglicht. Zusätzlich kann ein fixer Subventionsbetrag Unternehmen veranlassen, erstmalig F\&E-Projekte anzugehen, weil der Fixbetrag die erwartete Profitabilität eines Projekts von der Verlust- in die Gewinnzone wendet (extensiver Effekt). Einige Programme sehen eine anteilsmäßige Subvention von F\&E-Ausgaben vor, sodass sie ähnlich wie Steueranreize die F\&E-Grenzkosten senken. Das sollte die F\&E-Ausgaben sowohl entlang der intensiven als auch der extensiven Grenzen ausdehnen.

Empirische Studien zur Wirksamkeit von F\&E-Subventionen versuchen typsicherweise herauszufinden, ob die Zahlungen an die ausgewählten Unternehmen auch tatsächlich zu zusätzlichen eigenen Ausgaben führen (Additionalität). Die Auswahl der geförderten Projekte erfolgt jedoch nicht zufällig, sondern orientiert sich an Innovationsgehalt, Erfolgsaussichten und ähnlichen Kriterien. Die mit der Auswahl betrauten Personen haben oft einen Anreiz, Firmen zu fördern, die sowieso und auch ohne Förderung gute Wachstums- und Erfolgsaussichten haben. Das erhöht die Wahrscheinlichkeit, dass das Förderprogramm als Erfolg eingestuft wird, um die Reputation der Behörde und die berufliche Situation ihrer MitarbeiterInnen zu steigern. In der Folge unterscheiden sich die subventionierten Firmen systematisch von den nicht subventionierten. Die Analyse einer Gruppe ausschließlich geförderter Firmen würde den positiven Effekt von Subventionen überschätzen, weil diese auch ohne Förderung systematisch innovativer und erfolgreicher wären. Um den Auswahleffekt zu beseitigen und den Effekt der Subvention zu isolieren, sind Paarungen von Firmen mit und ohne Subventionen zu bilden, die sich in ihren sonstigen Charakteristiken möglichst ähnlich sind. Damit wird eine unverzerrte Schätzung der Wirksamkeit von F\&ESubventionen möglich (Klette u.a., 2000). 
Die neuere Arbeit von Jaffe und Le (2015) schätzt den Einfluss von F\&E-Subventionen auf den Innovationsoutput von neuseeländischen Firmen. Für die Innovationsmaße Patente und Marken stehen ihnen 298.000 Beobachtungen zu 97.000 Firmen über die Jahre 2005-2009 zur Verfügung. Für andere Maße des Innovationsoutputs haben sie Daten von 11.200 Firmen mit 26.400 Beobachtungen in Zwei-Jahres-Abständen zwischen 2005-2013. Um Selektionseffekte zu vermeiden und den Effekt der Subvention ohne Verzerrung zu isolieren, vergleichen sie geförderte Firmen mit anderen Firmen, die den ersteren so weit wie möglich ähneln, aber keinen F\&E-Zuschuss erhalten haben. Von allen beobachteten Unternehmen haben 5,3\% einen F\&E-Zuschuss in den letzten drei Jahren erhalten. Diese unterscheiden sich wesentlich von den anderen nicht geförderten Firmen. Geförderte Unternehmen sind innovativer, älter, größer (mehr MitarbeiterInnen) und stärker international orientiert. Nach dem Matching der geförderten mit nicht geförderten, aber sonst ähnlichen Unternehmen verschwinden diese Unterschiede in den Firmencharakteristiken, so dass lediglich der Unterschied im Innovationsoutput verbleibt. Das ermöglicht die Ermittlung des kausalen Effektes der F\&E-Subventionen auf den Innovationsoutput einer Firma.

In Neuseeland zeitigen F\&E Subventionen offenbar recht positive Effekte auf verschiedene Innovationsmaße. Zählt man jede Art von Innovation, dann haben Firmen ohne F\&EFörderung eine Wahrscheinlichkeit von 58\% für einen Innovationserfolg, d.h. 58\% (genau $57,7 \%$ ) der Firmen in der Kontrollgruppe konnten einen Innovationserfolg verbuchen. Bei vergleichbaren, aber geförderten Firmen steigt dieser Anteil um 5,7 Prozentpunkte auf 63,4\%, das ist ein Anstieg um 13\%. Die F\&E-Investitionen der Unternehmen können sich jedoch im Ausmaß und Risiko stark unterscheiden. So beträgt der Anteil von Prozessinnovationen in der Kontrollgruppe etwa 35\%. Eine F\&E-Subvention erhöht die Wahrscheinlichkeit einer Prozessinnovation um 5,3 Prozentpunkte, das ist um 15\% mehr. Demgegenüber steigt nach dem Zuschlag einer staatlichen F\&E-Subvention die Wahrscheinlichkeit einer Produktinnovation um 22\%, das entspricht einem Anstieg der Basiswahrscheinlichkeit (gleich 44\%) um 10 Prozentpunkte. Die Einführung eines neuen Produktes im Weltmarkt ist ein wesentlich ehrgeizigeres und riskanteres Vorhaben und hat daher eine Erfolgswahrscheinlichkeit von nur 12\%. Der Zuschlag einer F\&E-Förderung hat hier einen besonders starken Effekt und lässt die Wahrscheinlichkeit um 9,4 Prozentpunkte ansteigen, das ist eine Zunahme um 76\% relativ zur Kontrollgruppe, und löst ein Umsatzwachstum von 39\% aus. Diese Ergebnisse bleiben auch bei einer Variation der Schätzmethoden signifikant und robust.

Direkte Projektzuschüsse an Unternehmen, die bereits eine hohe F\&E-Expertise aufgebaut haben, entfalten stärkere Wirkungen als Förderprogramme, die auf einen erstmaligen Aufbau von F\&E-Aktivitäten zielen. Ein direkter Projektzuschuss an F\&E-aktive Unternehmen erhöht die Wahrscheinlichkeit einer Produktinnovation um 39\% bzw. um 17,4 Prozentpunkte ausgehend von 44\%. Dagegen vermag eine Unterstützung des erstmaligen Aufbaus von neuen F\&E-Fähigkeiten die Wahrscheinlichkeit einer Produktinnovation lediglich um 5 Prozentpunkte bzw. um $12 \%$ zu steigern. Die Analyse zeigt zudem, dass die Effekte bei kleinen und großen Unternehmen (mit weniger bzw. mehr als 50 Angestellten) recht ähnlich sind. Einer der wenigen größeren Unterschiede besteht darin, dass F\&E-Subventionen bei kleinen Unternehmen den Umsatz mit einem 50\%-Zuwachs wesentlich stärker ankurbeln als bei größeren Firmen, die in der Folge eine Umsatzsteigerung von 30\% verbuchen. 
Um den Innovationsertrag zu schützen, suchen die Unternehmen um Patentschutz an, angesichts der Kosten allerdings meist nur bei großen Innovationen mit hohem Wachstumspotenzial. Patente sind daher in der Kontrollgruppe der nicht geförderten Unternehmen mit 1,4\% ziemlich selten vertreten. Eine staatliche F\&E-Subvention steigert die Patentquote um 1,1 Prozentpunkte, was einer um 74\% höheren Wahrscheinlichkeit einer neuen Patentanmeldung entspricht. Große Unterschiede gibt es weder zwischen kleinen und großen Unternehmen noch zwischen solchen, die eine vorhandene F\&E-Kapazität ausdehnen oder erstmals neu aufbauen.

Ein vielbeachtetes Förderprogramm ist das US Small Business Innovation Research Program (SBIR), das in einem kompetitiven Auswahlverfahren Beiträge an kleine Firmen vergibt. Wallsten (2000) untersucht den Einfluss auf die F\&E-Ausgaben der Firmen, wobei er geförderte Firmen mit solchen vergleicht, die sich erfolglos um eine Subvention bewarben, und solchen, die die Bewerbungskriterien erfüllten, sich aber nicht bewarben. Die Resultate zeigen, dass geförderte Firmen schon vor der Förderung über mehr Beschäftigte verfügen, das Programm selbst aber keinen Einfluss auf die Zahl der Beschäftigten hat. Außerdem gibt es eine starke Substitution zwischen den staatlichen und privaten F\&E-Ausgaben. Die Beiträge reduzieren die firmeneigenen Aufwendungen praktisch eins zu eins. Diese Resultate beschreiben jedoch nur die kurzfristigen Auswirkungen des SBIR-Programms.

Lerner (1999) hingegen analysiert das Verhalten von SBIR-geförderten und nicht geförderten Firmen über eine Periode von 10 Jahren. Über diese Zeitspanne ergibt sich ein positiver Effekt auf Beschäftigung und Umsatz in den subventionierten Firmen. Diese hatten zudem eine höhere Wahrscheinlichkeit, zusätzliche Finanzierung in der Form von Wagniskapital zu erhalten. Diese positiven Auswirkungen waren aber nicht einheitlich, sondern beschränkten sich auf Gebiete mit einem höheren Angebot an Venture Capital und waren hauptsächlich in Hochtechnologie-Firmen zu beobachten. Zudem hatte die tatsächliche Höhe der Subvention keinen Einfluss auf die Resultate, sondern nur auf die Tatsache, dass ein Unternehmen eine solche erhalten hat. Das SBIR-Programm wirkte hauptsächlich als Zertifizierung der unterstützten Firmen. Andere Investoren wussten dadurch, dass diese Firmen ein relativ hohes Erfolgspotenzial versprachen, und waren eher bereit, auch in sie zu investieren.

Diese Zertifizierungsrolle und die Lockerung von Finanzierungsbeschränkungen legen nahe, dass F\&E-Subventionen in kleinen und großen Firmen unterschiedliche Wirkungen entfalten. Dies bestätigt auch die Studie von Lach (2002), die das größte F\&E-Subventionsprogramm Israels untersucht. In diesem werden Beiträge aufgrund eines kompetitiven Auswahlverfahrens vergeben, wobei sich die Firmen verpflichten, den Betrag eins zu eins um eigene F\&E-Aufwendungen zu ergänzen. Im Jahr, in dem sie die staatliche Zahlung erhalten, reduzieren kleine Firmen ihre eigenen Ausgaben in etwa um den dreifachen Betrag. Im Folgejahr jedoch erhöhen sie ihre F\&E-Investitionen um das 14-fache der erhaltenen Subvention, womit netto die Zunahme dem 11-fachen der Subvention entspricht. Bei großen Firmen ist allerdings keine signifikante Zunahme der F\&E-Ausgaben festzustellen. Da kleine Betriebe zudem nur einen geringen Teil der geförderten Firmen ausmachten, erbrachte das gesamte Programm keinen signifikanten Anstieg der privaten Innovationsausgaben.

Die empirische Forschung kommt insgesamt zu einem relativ breiten Konsens über die Wirksamkeit steuerlicher F\&E-Anreize. Bei direkten F\&E-Subventionen ist hingegen keine so klare Aussage möglich. Es gibt Programme, die zu einer deutlichen Erhöhung der F\&E- 
Investitionen in den geförderten Firmen führen. Andere sind dagegen mit kostspieligen Mitnahmeeffekten verbunden, sodass die Subventionen weitgehend nur eigene F\&EAusgaben ersetzen und keinen nennenswerten Nettoeffekt bewirken. Die Ausgestaltung der Subventionsprogramme, insbesondere deren Ausrichtung und die Verfahren zur Auswahl der geförderten Unternehmen, dürften einen wichtigen Einfluss auf die Wirksamkeit ausüben. Guellec und van Pottelsberghe (2000) untersuchen die relative Wirksamkeit von Steueranreizen und direkten Subventionen mit Daten von 1983 bis 1996 aus 17 OECDLändern. Die Ergebnisse deuten darauf hin, dass Steueranreize und Direktzahlungen Substitute sind. Gewährt ein Land sehr großzügige Steuererleichterungen, sind direkte Subventionen weniger stark wirksam, und umgekehrt. Für die Wirksamkeit staatlicher F\&EFörderung ist es zudem wichtig, dass eine Fördermaßnahme längerfristig in der gleichen Ausgestaltung bestehen bleibt. Die Unternehmen müssen sich darauf verlassen können, damit sie ihr Investitionsverhalten tatsächlich anpassen.

Die Förderung privater F\&E dürfte am wirksamsten sein, wenn ein Land Steueranreize nicht diskriminierend auf breiter Ebene einsetzt und Direktsubventionen nur für spezifische, begrenzte Förderziele benutzt. Steueranreize haben einen sehr breiten Einfluss, da sie allen F\&E-aktiven Unternehmen offenstehen. Wenn sie klar und einfach ausgestaltet sind und der Gesetzgeber eine konstante Anwendung gewährleistet, dann sind die Steuererleichterungen für die Unternehmen leicht planbar. Damit ist am ehesten gewährleistet, dass sie tatsächlich ihr Investitionsverhalten verändern. Gerade Österreich ist darauf angewiesen, für ausländische Unternehmen ein attraktiver Innovationsstandort zu bleiben. Einfachheit und Planbarkeit der Fördermaßnahmen stellen sicher, dass auch noch nicht im Inland angesiedelte Unternehmen die finanziellen Vorzüge der Maßnahmen genau abschätzen können. Im Gegensatz dazu ist eine Erwartungsbildung über die Teilnahme an Subventionsprogrammen deutlich schwieriger, da die Auswahlverfahren oft komplizierter sind und frühere Erfahrungen den Erfolg im Auswahlprozess deutlich erhöhen können.

\subsubsection{Andere steuerliche Aspekte}

Höherer Ertrag geht mit höherem Risiko einher. Gerade die grundlegendsten F\&EInvestitionen mit dem größten Ertrags- und Wachstumspotenzial sind auch dem höchsten Risiko ausgesetzt. In einer Wirtschaft, die sich der Technologiegrenze annähert und sich zum Technologieführer wandeln soll, müssen die Innovationen weitreichender werden und auch auf völlig neue Produkte und Prozesse zielen. Dafür müssen die Unternehmen ein wesentlich größeres Risiko nehmen und erwerben damit die Chance, die nächste Entwicklungsstufe vom Nischenanbieter und Hidden Champion zum weltweit agierenden Großkonzern zu gehen. Mehr Risiko braucht mehr Risikokapital. Risikokapital bereitzustellen ist allerdings mehr eine Aufgabe der Kapitalmärkte und weniger der Banken (Keuschnigg und Kogler, 2016). Auf dem Weg zum Technologieführer nimmt daher die Bedeutung der Kapitalmärkte relativ zur Bankenfinanzierung zu. Diese Entwicklung sollte auch das Steuersystem unterstützen, indem es die Bereitstellung von Risikokapital nicht länger steuerlich diskriminiert.

Auf der Ebene des Investors ist ein voller Verlustausgleich und Verlustvortrag im Rahmen der persönlichen Kapitalertragssteuer notwendig, um die Portfolioentscheidungen nicht zulasten riskanter Anlagen zu verzerren. Nur Erträge zu besteuern, aber sich an den Verlusten nicht $\mathrm{zu}$ beteiligen, ist eine Diskriminierung riskanter Anlageformen und behindert die 
Bereitstellung von Risikokapital. In Österreich ist es bei der Kapitalertragssteuer nur möglich, Verluste aus der Veräußerung von Kapitalvermögen mit Überschüssen aus gleichartigen Kapitalvermögen auszugleichen. Ein Ausgleich von Verlusten aus Aktienvermögen mit Zinsen auf Spareinlagen oder gar mit anderen Einkunftsarten wie Lohneinkommen ist nicht möglich. Ebenso wenig ist ein Verlustvortrag möglich. Nur die Verlustanteile von stillen Gesellschaftern können vorgetragen und mit späteren Gewinnen aus derselben stillen Beteiligung verrechnet werden (Doralt, 2012, S. 15-16). Die Beschränkung des Verlustausgleichs wird oft mit dem Schutz des Steueraufkommens motiviert. Allerdings sind die erwarteten Erträge aus riskanten Anlagen um eine marktübliche Risikoprämie höher als z.B. der Ertrag einer festverzinslichen Anleihe. Folglich sind auch die Einnahmen aus der Kapitalertragsteuer höher als bei einer sicheren, aber weniger rentierlichen Veranlagung.

Eine innovationsfreundliche Besteuerung sollte den Unternehmen die Finanzierung mit risikotragendem Eigenkapital erleichtern. Weil in praktisch allen Ländern die Zinsen für Fremdkapital, aber nicht für Eigenkapital steuerlich abzugsfähig sind, setzt die Besteuerung einen sehr großen Anreiz zur Verschuldung. 24 Praktisch alle Steuerreformvorschläge aus der Wissenschaft stellen dagegen auf Finanzierungsneutralität ab und fordern eine Gleichbehandlung von Eigen- und Fremdkapital.25 Eine Möglichkeit ist, nicht nur die Zinsen auf Fremdkapital, sondern auch eine marktübliche Verzinsung des Eigenkapitals steuerlich abzugsfähig zu machen. Dadurch würden langfristig die Einnahmen der Körperschaftssteuer erheblich schrumpfen. Allerdings sollte die Abzugsfähigkeit sich auf neu ausgegebenes Eigenkapital beschränken und nicht das historisch vorhandene Eigenkapital im Nachhinein begünstigen. Damit würde der Einnahmenausfall nur langsam in dem Ausmaß anfallen, wie neues Eigenkapital gebildet wird. Die Reform würde Investitionen und Standortattraktivität fördern und starke Wachstumsimpulse auslösen. Sie begünstigt vor allem junge innovative Wachstumsunternehmen, die ein höheres Risiko tragen und daher mehr Eigenkapital brauchen. Aus demselben Grund würde sie auch die internationale Spezialisierung der Wirtschaft auf innovative Branchen begünstigen (Egger und Keuschnigg, 2015).

24 Siehe Keuschnigg (2016) für einen Überblick zur empirischen Forschung. 25 Vgl. z.B. Mirrlees (2011) und U.S. President's Advisory Panel on Federal Tax Reform (2006). 


\subsection{Risikokapital und Innovation}

Ein Land, das zu den technologisch führenden Ländern vorstoßen will, darf sich nicht auf eine Nischenstrategie beschränken, sondern muss mehr und größere Innovationen schaffen. Die radikalsten und riskantesten Innovationen liefern die höchsten Wachstumschancen und stammen oft von risikoreichen Start-ups. Wagniskapital (Venture Capital, Private Equity, Business Angels) ist auf die Finanzierung innovativer Wachstumsunternehmen spezialisiert. Angesichts des großen Ausfallsrisikos sind besonders viele Gründungen nötig, damit einige wenige zu wirklich großen Unternehmen heranwachsen.

\section{Abbildung 29: Wagniskapital für Unternehmen, 2014 oder letztes verfügbares Jahr}

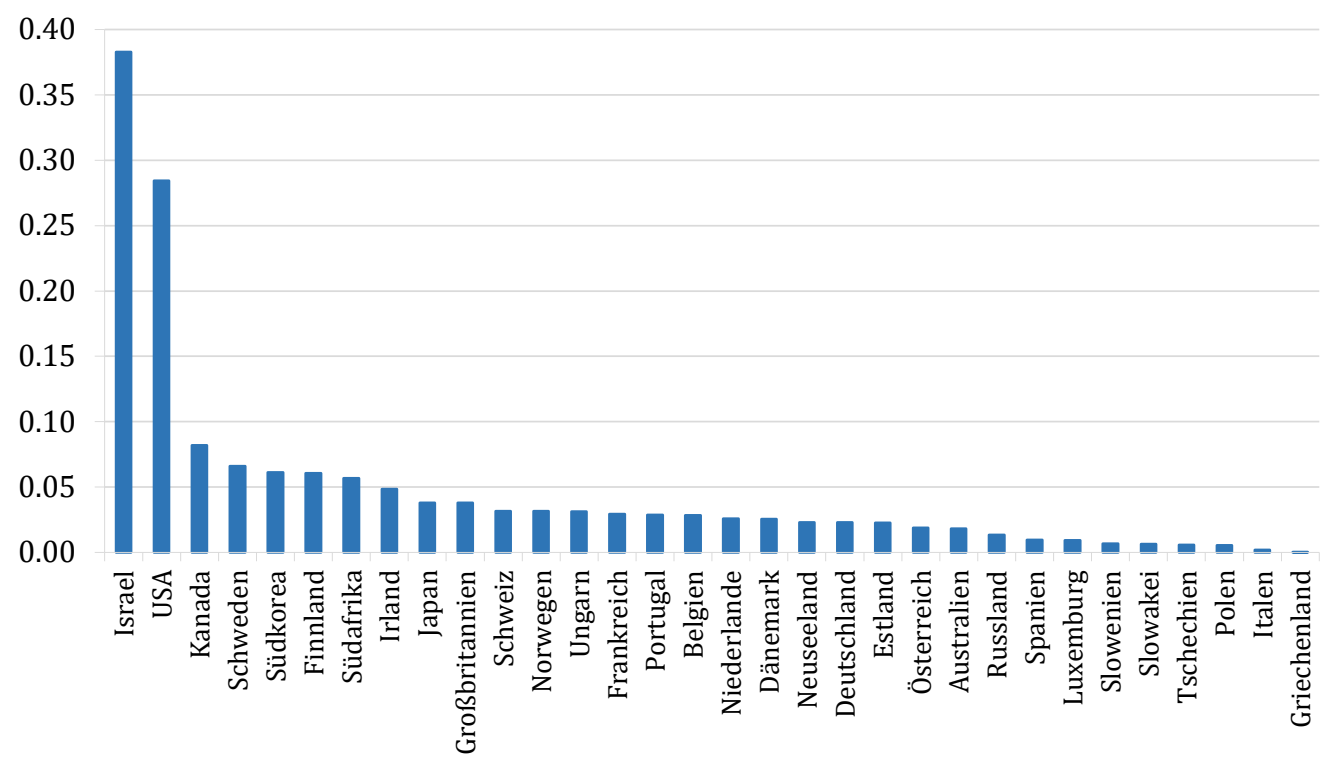

Quelle: OECD (http://www.oecd-ilibrary.org/sites/entrepreneur_aag-2015-en/07/01/g7-1.html), eigene Darstellung

Die Aufgabe des Wagniskapitals liegt darin, höchst riskante, aber sehr rentable Investitionsprojekte zu finanzieren, die sonst mangels Finanzierung nicht zustande kämen, und innovative Wachstumsunternehmen unterstützen, ihr Potenzial besser auszuschöpfen, als es sonst möglich wäre.26 Indem eine aktive Wagnisfinanzierung über die ersten kritischen Phasen hinweghilft, bereitet sie die Kreditfinanzierung und den Zugang zum Kapitalmarkt in späteren Phasen vor. Wagniskapital vervollständigt den Finanzplatz und stellt Beteiligungsfinanzierung bereit, wo Banken und andere Akteure keine Kredite vergeben

26 Vgl. Da Rin u.a. (2013) zum Stand der Forschung. Wagniskapital stärkt das innovationsgetriebene Wachstum (Keuschnigg, 2004a) und fördert die Spezialisierung auf innovative Industrien (Egger und Keuschnigg, 2015). Kortum und Lerner (2000) schätzten für die USA, dass ein Dollar an F\&E Ausgaben in wagnisfinanzierten Unternehmen mehr Patente und radikalere Innovationserfolge erzielten wie dieselben Ausgaben in Firmen ohne Wagnisfinanzierung. Demnach entfielen 1998 etwa 14 Prozent der industriellen Innovation in den U.S.A. auf wagnisfinanzierte Firmen, obwohl diese nur 3 Prozent der gesamten F\&E Ausgaben tätigten. Lerner u.a. (2011) finden, dass Patente, die in den 3 folgenden Jahren nach Hinzutritt eines Wagnisfinanziers angemeldet werden, um 79\% mehr Zitate erzielen wie jene Patente, die im selben Jahr der Wagnisbeteiligung angemeldet und daher davor erarbeitet wurden. Das wirtschaftliche Potenzial der Patente steigt. 
können. Angesichts der Rolle des Wagniskapitals in der Finanzierung der innovativsten und riskantesten Gründungen ist das geringe Volumen in Österreich (vgl. Abbildung 29) ein starkes Innovationshemmnis.

Innovative GründerInnen sind auch dem größten Risiko ausgesetzt. Ein neues Angebot ist auf dem Markt noch nicht getestet. Innovative Start-ups sind in der ersten Phase meist auf die technologischen Herausforderungen der Produktentwicklung fokussiert. Aufgrund ihrer kurzen Geschichte hatten sie wenig Gelegenheit, unternehmerische Erfahrung zu sammeln und ein professionelles Management einzurichten. Dies ist umso verhängnisvoller, als die häufigste Ursache für das Scheitern in Managementfehlern unterschiedlichster Art liegt. Angesichts der kurzen Lebenszeit konnten junge Start-ups noch wenig Eigenkapital bilden. Daher ist es kein Zufall, dass innerhalb der ersten drei bis fünf Jahre etwa die Hälfte der Unternehmen scheitert und aufgeben muss.

Eine reine Kreditfinanzierung scheidet meist aus, wenn sich die Banken angesichts eines unerprobten Geschäftsmodells und fehlender Sicherheiten einem überaus hohen Risiko gegenüber sehen, und wenn der Unternehmenserfolg und damit die Bedienung des Kredits zu sehr von der Geschäftsidee und der Kompetenz der Gründerin bzw. des Gründers abhängen. Die Banken haben nicht die Kapazität, um neben der Finanzierung auch intensive strategische Beratung und Managementkontrolle zu leisten, um den Erfolg und damit die sichere Rückzahlung der Kredite positiv zu beeinflussen. Banken müssen viele Unternehmen betreuen, während Wagnisfinanziers wegen der hohen Beratungsintensität nur ganz wenige Firmen finanzieren. Innovative Gründungen brauchen risikotragendes Beteiligungskapital sowie aktive strategische Beratung und Überwachung aus einer Hand.

Die Finanzierung erfolgt typischerweise mit Eigenkapital oder eigenkapitalähnlichen Instrumenten wie Wandelschuldverschreibungen und wird auf mehrere Runden aufgeteilt, wobei die nächste Finanzierungstranche vom Erreichen wichtiger Meilensteine der Unternehmensentwicklung abhängt. Indem sie Eigenkapital zur Verfügung stellen, nehmen die Wagnisfinanziers den Unternehmern einen erheblichen Teil des Risikos ab. Mehr Eigenkapital und das Engagement eines professionellen Investors ermöglichen meist zusätzliche Finanzierung mit Bankkrediten, die sonst nicht verfügbar wäre, und damit mehr Investition. Angesichts des Risikos sichern sich die Finanziers mit speziellen Vertragsklauseln weitere Rechte über die Beteiligung hinaus, damit sie im Notfall aktiv eingreifen und ihre Rendite retten können.27 Indem sie sich mit Eigenkapital anteilig an den Gewinnen und Verlusten beteiligen, erhalten sie starke Anreize, sich mit wertsteigernden Beratungs- und Kontrolltätigkeiten zu engagieren und aktiv auf den größtmöglichen Unternehmenserfolg hinzuarbeiten, um den eigenen Beteiligungsertrag weiter zu steigern und Fehlentwicklungen vorzubeugen. Ein Partner einer Finanzierungsgesellschaft kann nur wenige Portfoliofirmen betreuen, wenn die Qualität der begleitenden Unterstützung erhalten bleiben soll.28 Die aktive Unterstützung der Beteiligungsunternehmen macht die Wagnisfinanzierung zu einem

27 Die Studie von Kaplan und Strömberg (2000) ergab für die USA, dass Wagnisfinanziers oft 50\% der Gewinnanteile halten und die GründerInnen selbst etwa 30\%. Die Risikokapitalisten sitzen regelmäßig im Aufsichtsrat und haben in 25\% der Fälle die Mehrheit der Stimmen. Die Finanzierungsverträge sehen meist zusätzliche Eingriffs- und Kontrollrechte vor.

28 Vgl. die Diskussion in Kanniainen und Keuschnigg (2004) und Keuschnigg (2004b). 
zeitintensiven und teuren Geschäft. Aus diesen Gründen ist sie wesentlich teurer als ein Bankkredit, aber die Unternehmen erhalten auch weit mehr als nur Kapital.

Wagniskapital entschärft zwei Marktstörungen in der Finanzierung innovativer Start-ups: Zugang zu Kapital und Managementunterstützung bei mangelnder unternehmerischer Erfahrung. Erstens ermöglicht Wagniskapital die Realisierung von potenziell sehr rentablen Projekten, die sonst keine Finanzierung erhalten würden. So können Einkommens- und Beschäftigungsmöglichkeiten genutzt werden, die sonst brachliegen würden. Zweitens unterstützen die strategische Beratung und Managementkontrolle die Beteiligungsfirmen, ihr Potenzial besser auszuschöpfen und mehr Wachstum zu realisieren. Firmen, die Wagniskapital erhalten, wachsen schneller, werden größer und schaffen mehr Jobs als andere vergleichbare Unternehmen. Der wertsteigernde Einfluss macht sich bemerkbar, indem wagnisfinanzierte Firmen radikalere Innovationsstrategien verfolgen und mehr Patente anmelden; den Markteintritt beschleunigen, um eine dominierende Marktstellung zu erlangen; und schneller Management, Marketing und Personalwesen professionalisieren.29 Die GründerInnen nehmen häufig einen Verlust an Unabhängigkeit durch unbequemen Fremdeinfluss wahr, wenn die Wagnisfinanziers ihre Kontrollrechte und Eingriffsmöglichkeiten nutzen. Dies hat jedoch die positive Folge, frühzeitig vermeidbare Ausfälle verhindern und mögliche Fehlentwicklungen bezüglich des weiteren Wachstums korrigieren zu können.

Der wertsteigernde Einfluss eines Wagnisfinanziers, der seine unternehmerische Erfahrung, Marktkenntnisse, Kontakte und Netzwerke einbringt, wirkt auf andere Kapitalgeber vertrauensbildend und ermöglicht oft zusätzliche Bankkredite. Hochberg u.a. (2014) zeigen empirisch, dass Wagniskapital den Zugang zu Bankkrediten verbessert. Nach der ersten Beteiligung eines Wagnisfinanziers steigt der Finanzierungsanteil von anderen Krediten von 4,6 auf 8,3\% an, also beinahe auf das Doppelte. Bei Wagnisfinanziers mit besonders hoher Reputation ist dieser Effekt noch wesentlich stärker. 30 Je größer die eigene unternehmerische Erfahrung, Marktkenntnisse und Reputation der Wagnisfinanziers sind, desto eher macht sich der wertsteigernde Einfluss bemerkbar, und desto eher gelingt es den Finanzierungsgesellschaften, die Projekte mit besonders hohem Potenzial aufzuspüren.

Sorensen (2007) zeigt, dass der Wagnisfinanzier mit der geringsten Erfahrung in seinem Datensatz ein Portfoliounternehmen mit einer Wahrscheinlichkeit von 21,4\% an die Börse bringt, während der Investor mit der größten Erfahrung dasselbe mit einer Wahrscheinlichkeit von 38,9\% schafft, das ist ein Anstieg um 82\%. Die bessere Performance von wagnisfinanzierten Firmen im Vergleich zu anderen Start-ups lässt sich nach Sorensen (2007) zu etwa einem Drittel auf strategische Beratung und Kontrolle und zu zwei Drittel auf eine bessere Projektauswahl zurückführen, indem Wagnisfinanziers von vornherein

29 Hellmann und Puri (2000) zeigen empirisch, dass aktive Wagnisfinanziers starken Einfluss auf die Entwicklung der Firma nehmen. Nach dem Eintritt eines Wagnisfinanziers steigt im Vergleich zu anderen Firmen die Wahrscheinlichkeit, dass professionelles Personal eingestellt wird, im Marketing um 26\%, im Finanzwesen und administrativen Bereich um 44\% und im Management um 15\%. Es wird etwa 2,3 Mal so wahrscheinlich, dass eine Firma die Gründerin bzw. den Gründer durch eine professionelle Managerin bzw. einen professionellen Manager ersetzt, wenn das weitere Wachstum es erfordert. Die Wahrscheinlichkeit, als erste Firma ein neues Produkt auf dem Markt einzuführen, erhöht sich um den Faktor 1,8. Die Ergebnisse von Puri und Zarutskie (2012) gehen in dieselbe Richtung.

30 Hellmann u.a. (2008) zeigen, dass Banken eher Kredite an Firmen geben, in die sie schon in der Wagnisphase investiert haben, und auch zu deutlich besseren Bedingungen als bei jenen Unternehmen, die niemals Wagnisfinanzierung hatten. 
innovativere Projekte identifizieren und finanzieren. Das sind starke Hinweise, dass ein aktiver Markt für Wagniskapital den Gründungen zu mehr Erfolg und der Wirtschaft zu mehr Wachstum und Produktivität verhelfen kann.

Abbildung 30: Wagniskapital und Börsenkapitalisierung in \% des BIP, 2014

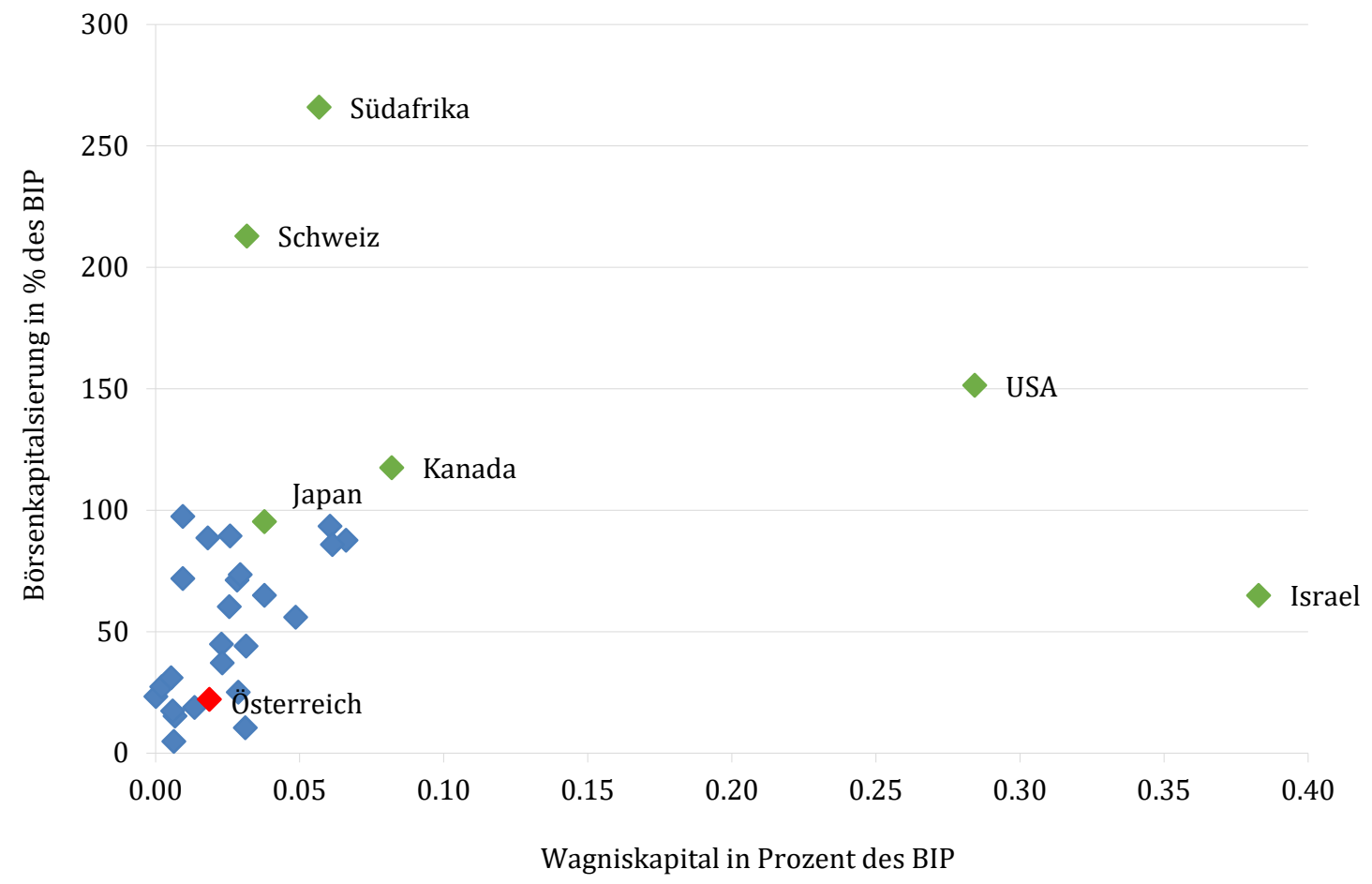

Anmerkung: Angaben für 2014 oder letztes verfügbares Jahr

Quelle: OECD für Wagniskapital, Weltbank für Börsenkapitalisierung

Die Schwierigkeiten im Zugang zu Kapital und der strategische Beratungsbedarf sind in der risikoreichen Anfangsphase am größten und lösen sich im Laufe einer erfolgreichen Firmenentwicklung immer weiter auf. Die Unternehmen bauen Risiken ab, professionalisieren sich und werden besser von außen überprüfbar, bilden mehr Eigenmittel und können zunehmend Sicherheiten für eine Kreditfinanzierung bereitstellen. Die teure Wagnisfinanzierung kann durch Kapitalmarktfinanzierung und Bankkredite abgelöst werden. Aus diesen Gründen werden Beteiligungsfonds von vornherein nur mit beschränkten Laufzeiten aufgelegt. Der Ausstieg erfolgt bei besonders erfolgreichen Beteiligungen im Zuge eines Börsengangs, womit die Gesellschaften am ehesten gute Preise für ihre Anteile erzielen. Alternativ wird die Beteiligung an andere Unternehmen oder Investoren verkauft oder muss ganz abgeschrieben werden. Die besseren und profitableren Ausstiegsmöglichkeiten mit einer liquiden Börse für junge Technologieunternehmen begünstigen die Entwicklung einer aktiven Wagniskapitalbranche.31 Das geringe Finanzierungsvolumen des Kapitalmarkts und

31 Eine liquide Börse erleichtert den Ausstieg bei reiferen Unternehmen und setzt Kapital und Managementressourcen der Wagnisfinanziers für Reinvestitionen bei neuen Start-ups frei. Da Rin u.a. (2006) zeigen, dass der Zugang zu einem aktiven 
besonders der Börse (vgl. Abbildung 30) stellt ein großes Innovationshemmnis für Österreich dar (vgl. Keuschnigg und Kogler, 2016, zum Finanzplatz Österreich).

\subsection{Insolvenzrecht}

Neue Produkte und Prozesse müssen sich in der Praxis und bei den Nachfragern bewähren. Je radikaler die Innovation, desto grösser ist das Risiko. Wenige Vorhaben und Investitionen sind derart riskant wie innovative Unternehmensgründungen. Etwa die Hälfte der neu gegründeten Unternehmen ist nach 5 Jahren nicht mehr aktiv. Ein Großteil der wagnisfinanzierten Gründungen sind Totalverluste und nur etwa 3-5 von 100 sind ein wirklicher Erfolg und schaffen es bis zur Börseneinführung (vgl. Kerr u.a., 2014). Die Gründer müssen also das Scheitern mit hoher Wahrscheinlichkeit von vornherein einkalkulieren. Für die Risikobereitschaft und die Neigung zur Unternehmensgründung ist daher die Behandlung im Insolvenzfall von großer Bedeutung. Die Ausgestaltung des Insolvenzrechts spielt daher für die Häufigkeit von Insolvenzen, den Umfang der Kapitalvernichtung und den Neueinsatz der freigesetzten Ressourcen Arbeit und Kapital und für die Verfügbarkeit von Risikokapital eine wichtige Rolle.

Das Insolvenzrecht spezifiziert ein Verfahren, wie die Ansprüche der Gläubiger erfüllt werden, wenn eine Privatperson oder ein Unternehmen seine finanziellen Verpflichtungen nicht mehr erfüllen kann (vgl. White, 2001, und Bris, 2008, für einen ökonomischen Überblick). Dabei sind drei Aspekte wichtig. Erstens legt das Insolvenzrecht Regeln fest, wie die Ansprüche unabhängig von ihrer Fälligkeit simultan behandelt werden. Es bestimmt, welche Vermögenswerte zur Abgeltung der Ansprüche herangezogen und ob auch zukünftige Einkommen zur Rückzahlung von Schulden gepfändet werden können. Zweitens regelt es, wie die vorhandene Insolvenzmasse auf die Gläubiger aufzuteilen ist. Diese Regeln müssen auch verhindern, dass besser informierte und schnell handelnde Anspruchsberechtigte sich vorzeitig bedienen, damit die Insolvenzmasse verkleinern und die übrigen Gläubiger schädigen. Das Insolvenzrecht bestimmt also sowohl die Größe als auch die Art der Aufteilung der verfügbaren Insolvenzmasse. Drittens legt das Insolvenzrecht auch die Sanktionierung des Schuldners fest, der seinen Verpflichtungen nicht mehr nachkommt. Die Bekanntmachung einer Insolvenz stigmatisiert den Schuldner, mindert seine Kreditwürdigkeit und oft auch sein berufliches Fortkommen. Das Insolvenzrecht muss auch festlegen, wie lange der Schuldner für seine Verpflichtungen haftet bzw. wie schnell die Verpflichtung zur Rückzahlung aufgehoben wird.

Bei einer Liquidation oder Abwicklung gibt es eine klare Haftungsreihenfolge bzw. Prioritätenregel für die Aufteilung der Insolvenzmasse auf die Gläubiger. Zuerst müssen besicherte und vorrangige Schulden bedient werden, deren Seniorität vertraglich vereinbart ist. Soweit die verbleibende Insolvenzmasse noch reicht, werden danach die ungesicherten Schulden bedient. Ist nicht genügend verwertbares Vermögen vorhanden, dann erhalten alle Gläubiger nur einen Anteil bzw. eine Quote und müssen den anderen Teil ihrer Forderung abschreiben. Die Eigentümer werden je nach ihrem Beteiligungsanteil zuletzt bedient, sofern die Insolvenzmasse nicht schon vorher aufgebraucht ist. Meist erleiden sie einen Totalverlust

Börsensegment für junge Technologieunternehmen die Wagniskapitalinvestitionen besonders in der Frühphase und im Hochtechnologiebereich im Vergleich zu anderen Standorten um etwa 10\% steigern könnte. 
auf ihre Beteiligung. Die beschränkte Haftung schützt das Arbeitseinkommen der Eigentümer und ihr übriges Privatvermögen, sofern sie es nicht als Sicherheit für Kredite zur Verfügung gestellt haben. Klarerweise bestimmt die Behandlung in einer Insolvenz das Risiko der Kapitalgeber und damit die Risikoprämie, die sie zur Entschädigung ihrer Risikobereitschaft fordern müssen. Vorrangige Schuldpapiere oder besicherte Kredite sind dem geringsten Risiko ausgesetzt und werfen die niedrigsten Zinsen ab. Nachrangige Schulden werden in einer Insolvenz meist nur mehr teilweise bedient, tragen ein höheres Risiko und müssen deshalb höhere Zinsen zahlen. Beteiligungskapital ist dem höchsten Risiko ausgesetzt und muss im Insolvenzfall meist einen Totalverlust verbuchen. Zur Kompensation der Risikotragung muss seine Rendite eine wesentlich höhere Risikoprämie enthalten.

Nach einer Zahlungsunfähigkeit ist alternativ zur Abwicklung auch eine Restrukturierung bzw. Sanierung eines Unternehmens möglich. Bei einer Abwicklung werden alle Vermögenswerte verwertet und gehen vollständig in die Insolvenzmasse ein, während sie bei einer Sanierung für den weiteren Betrieb im Unternehmen bleiben. Die Sanierung erfordert eine Einigung aller Gläubiger mit den Eigentümern über einen Schuldenschnitt und einen Rückzahlplan für den restlichen Teil der Verpflichtungen. Der Schuldenschnitt muss so groß ausfallen, dass die Sanierung des Unternehmens mit den entsprechenden Auflagen tatsächlich gelingt und wenigstens die Rückzahlung der verbleibenden Verpflichtungen möglich wird. Da bei der Restrukturierung auch die Eigentümer zustimmen müssen, kommt es meist zu einer freiwillig vereinbarten Abweichung von der Prioritätenregel, bei der die Eigentümer besser als bei der Liquidation abschneiden, um ihre Anreize und ihr Interesse am Gelingen der Restrukturierung zu erhalten. Volkswirtschaftlich geht es bei der Entscheidung zwischen Abwicklung und Sanierung letzten Endes darum, wo die Vermögenswerte mehr Einkommen erwirtschaften können, entweder durch die weitere Nutzung im fortgeführten Unternehmen oder durch Verkauf an andere Eigentümer (z.B. Konkurrenten) mit besseren Verwendungsmöglichkeiten. Kapital soll dort zum Einsatz kommen, wo es den höchsten Ertrag erwirtschaftet. Wenn bei einer Sanierung die Vermögenswerte mehr Einkommen abwerfen als bei Übertragung an andere Nutzer, dann steht prinzipiell auch mehr Einkommen zur Schuldentilgung zur Verfügung. So können sich auch die Gläubiger besser stellen.

Anders als bei Unternehmen ist bei einer Privatinsolvenz prinzipiell nur eine Sanierung möglich, denn eine Privatperson kann nicht abgewickelt werden. Nur das Finanz- und Sachvermögen ist verwertbar. Der wichtigste Vermögenswert ist jedoch die eigene Arbeitskraft, die nicht verkauft werden kann. Dem steht prinzipiell nicht entgegen, dass künftiges Arbeitseinkommen teilweise gepfändet und zur Begleichung der Schulden herangezogen wird. Das Verfahren zur Privatinsolvenz ist für kleine Unternehmen sehr relevant, weil die Inhaber von Personenunternehmen mit ihrem gesamten Vermögen haften und die Inhaber von kleinen Kapitalgesellschaften oft mit ihrem Privatvermögen die Schulden des Unternehmens garantieren. Indem das Insolvenzrecht einen Teil des Privatvermögens freistellt, d.h. vor dem Zugriff der Gläubiger schützt, und auch die Pfändung von künftigen Lohneinkommen begrenzt, erzielt es einen wohlfahrtssteigernden Versicherungseffekt. Die Konsequenzen des Scheiterns werden gemildert, aber dafür müssen die Schuldner höhere Zinsen bezahlen, solange sie Erfolg haben und zahlungsfähig sind. Auf diese Weise wird Einkommen von den günstigen zu den ungünstigen Lebenslagen verschoben, um allzu große 
Einbrüche zu vermeiden und den Wohlstand zu glätten. Ein solcher Versicherungseffekt entlastet auch den Sozialstaat.

Das Insolvenzrecht soll den Schuldnern ein akzeptables zukünftiges Fortkommen ermöglichen und muss dabei einen großen Interessensgegensatz zwischen Gläubigern und Schuldnern auflösen. Diese Abwägung fällt in verschiedenen Ländern ganz unterschiedlich aus. Manche gewähren hohe Freibeträge für das Privatvermögen und schließen, um einen Neustart zu unterstützen, das zukünftige Lohneinkommen von der Haftung aus, so dass nur das Finanz- und Sachvermögen über die Freigrenze hinaus verwertbar ist. Andere Länder setzen auf einen stärkeren Gläubigerschutz, halten die Freigrenzen für das Privatvermögen gering und beziehen auch das zukünftige Arbeitseinkommen über einen langen Zeitraum in die Rückzahlungspflicht mit ein. Die Betonung der Gläubigerrechte beeinträchtigt jedoch das berufliche Fortkommen der Schuldner und erschwert einen Neubeginn. Die Regelungen des Insolvenzrechts unterscheiden sich recht stark, sowohl zwischen den Bundesstaaten der U.S.A. als auch zwischen den Mitgliedsländern der EU.32

Das Insolvenzrecht hat wichtige Auswirkungen auf das wirtschaftliche Verhalten aller Beteiligten und kann damit den Strukturwandel in einer innovativen Wirtschaft erleichtern oder bremsen. Wenn das Insolvenzrecht gegenüber den Schuldnern zu weich ist, kommt es tendenziell häufiger und früher zu Insolvenzen. In den vielen Insolvenzverfahren wird zu viel Vermögen vernichtet und die Vermögenswerte gehen an andere Nutzer über, obwohl die ertragreichste Verwendung in der Fortführung des Unternehmens nach einer Restrukturierung läge. Ein zu weiches Insolvenzrecht verleitet die Manager und Unternehmer tendenziell zu einem zu riskanten Investitionsverhalten. In Kapitalgesellschaften mit beschränkter Haftung gehen die Gewinne vollständig an die Eigentümer, während sie für die Verluste nur bis zum Wert ihrer Beteiligung haften. Bei riskanteren Projekten werden sowohl Gewinne als auch Verluste grösser. Die Eigentümer bevorzugen riskantere Projekte, weil alle zusätzlichen Gewinne an sie selbst gehen, während sie im Insolvenzfall nicht mehr als ihr Beteiligungsvermögen verlieren können und die zusätzlichen Verluste bei den Gläubigern hängen bleiben. Aus demselben Grund mindert ein weiches Insolvenzrecht auch die Managementanstrengungen und begünstigt einen Anstieg der Insolvenzen.

Bei einem so riskanten Vorhaben wie einer Unternehmensgründung müssen schon von Beginn an die Folgen einer möglichen Insolvenz einkalkuliert werden. Ein besonders schuldnerfreundliches Insolvenzrecht mindert die Kosten eines Fehlschlags, fördert den Mut

32 Das österreichische Insolvenzrecht ist in der Insolvenzordnung geregelt, die 2010 reformiert wurde. Es unterscheidet ähnlich wie vorhin zwischen Sanierungs- und Konkursverfahren. Bei einem Sanierungsverfahren ohne Eigenverantwortung geht die Verfügungsgewalt über das Unternehmen auf einen gerichtlich bestellten Insolvenzverwalter über, bei einem Verfahren mit Eigenverantwortung leitet der Schuldner weiterhin unter gewissen Auflagen das Unternehmen. In beiden Fällen erhält der Schuldner nach Erfüllung des Sanierungsplans die volle Autonomie zurück und erlöschen die Restschulden. Im Konkursverfahren erlischt die Gewerbeberechtigung nach der geordneten Abwicklung durch den Insolvenzverwalter. Privatinsolvenzen erfolgen nach dem Schuldenregulierungsverfahren in drei Fällen. Erstens, die Gläubiger können einem Sanierungsplan zustimmen, mit dem eine Mindestquote von 20\% innerhalb von fünf Jahren zurückzuzahlen ist. Wenn die Gläubiger nicht zustimmen, kommt es zweitens zum Privatkonkurs mit einem gerichtlich festgelegten Zahlungsplan über maximal 7 Jahre (ohne Mindestquote), wonach das Privatvermögen verwertet und auch das künftige Einkommen einschließlich Lohn herangezogen wird. Wenn die Gläubiger nicht zustimmen, startet das Abschöpfungsverfahren mit Restschuldbefreiung nach maximal 7 Jahren. Das Verfahren endet erfolgreich, wenn innerhalb von 3 Jahren mindestens 50\% und innerhalb von 7 Jahren mindestens 10\% der Gläubigerforderungen befriedigt werden. 
zum unternehmerischen Risiko, und stärkt die Neigung zur Unternehmensgründung. Der Vorteil der Schuldner ist jedoch der Nachteil der Gläubiger. Wenn die Häufigkeit der Insolvenzen und die dabei auftretenden Verluste zunehmen, müssen die Gläubiger höhere Zinsen fordern und werden öfter die Finanzierung ganz versagen, wenn sie im Insolvenzfall hohe Vermögensverluste erwarten müssen. Die Verteuerung und Verknappung der Finanzierung bremsen das Unternehmenswachstum und die Neigung zur Gründung. Dagegen mindert ein scharfes Insolvenzrecht das Risiko der Gläubiger und steht für einen hohen Investoren- und Gläubigerschutz, der die Verfügbarkeit von Risikokapital und damit die Chancen für eine Innovationsfinanzierung zu geringen Kosten steigert.

Eine umfangreiche empirische Literatur bestätigt die genannten Zusammenhänge. Zunächst stellt man erhebliche Insolvenzkosten fest. Nach White (2011) machen die direkten Kosten für Anwaltshonorare und administrative Gebühren in den U.S.A. im Durchschnitt 3-4\% der Vermögenswerte aus. Dazu kommen indirekte Kosten wie z.B. missbräuchliche Abzweigung von Vermögenswerten, Verlust von wichtigem Personal, verschlechterter Zugang zu Finanzierung und entgangene Geschäfte aufgrund von erhöhtem Misstrauen von Kunden und Lieferanten. Diese sind deutlich grösser und können im Extremfall bis zu 20 Mal höher ausfallen wie die direkten Insolvenzkosten. Bei Sanierungen nach einer Einigung der betroffenen Parteien kommt es in den meisten Fällen zu einer Abweichung von der Prioritätenregel für die Haftung, wonach die Gläubiger auf einen Teil ihrer Forderungen verzichten und auch die Alteigentümer am Sanierungserfolg teilweise partizipieren. Diese Abweichung beträgt etwa 3-7\%, d.h., wenn die Gläubiger noch 100`000 als Rückzahlung erhalten, dann ist nach dem Restrukturierungsplan für die Eigentümer typischerweise ein Dividendenanspruch von 3 bis 7 Tausend vereinbart.

Daneben besteht ein systematischer Einfluss auf wichtige Verhaltensanreize schon lange vor einer Insolvenz. Ein weiches Insolvenzrecht mit großzügigen Freibeträgen und einer Freistellung zukünftiger Arbeitseinkommen hat einen starken Einfluss auf die Zahl der Insolvenzanmeldungen. Auch private Haushalte können einen Konkursantrag stellen, um ihre Zahlungsunfähigkeit geordnet zu lösen und einen Zahlungsausfall mit Zwangsvollstreckung zu vermeiden. Mit der Reform des Insolvenzrechts im Jahr 2005 haben sich die Kosten für ein Konkursverfahren wegen strengerer Dokumentations- und Beweispflicht stark erhöht. So sind die direkten Kosten von 600 auf 2‘500\$ bei einer Insolvenz nach Chapter 7 und von 1‘600 auf 3‘500\$ bei einer Sanierung nach Chapter 13 gestiegen. Allein die Anwaltskosten, die etwa drei Viertel der gesamten Kosten ausmachen, haben um etwa 35\% zugenommen (Albanesi und Nosal, 2015). In der Folge ist die Anzahl der Konkursanträge stark gefallen, während die Zahlungsausfälle und Zwangsvollstreckungen stark zugenommen haben. Li, White und Zhu (2011) haben im Bereich der Eigenheimfinanzierungen eine Zunahme der Zahlungsausfälle und Betreibungen bei sogenannten Prime- und Subprime-Hypotheken um 23 bzw. 14\% geschätzt, während Albanesi und Nosal (2015) spiegelbildlich einen ähnlich großen Rückgang der Konkursanträge geschätzt haben.

Während vor der Reform die Eigenheimbefreiungen im Konkursverfahren in den Bundesstaaten von 0 bis unbegrenzt variierten, wurde mit der Reform 2005 eine neue Obergrenze von 125‘000\$ eingeführt. Die Befreiung hat den Erwerb von Eigenheimen stark begünstigt. Corradin u.a. (2016) schätzen, dass eine Erhöhung der Eigenheimbefreiung von 0 auf etwa 350`000\$ (das entspricht vor der Reform einer Standardabweichung zwischen den 
Bundesstaaten) den Anteil des Netto-Immobilienvermögens (Gesamtwert minus Schulden) am gesamten Finanz- und Sachvermögen um 22\% steigert. Die Forscher argumentieren, dass ein solches Portfolio nicht effizient ist und die Haushalte einem zu großen Risiko aussetzt. Andererseits bieten Befreiungen im Konkursverfahren mehr Sicherheit bei großem beruflichem Risiko und fördern die Neigung zur Gründung, wenn die Unternehmer ihr Eigenheim auch bei einer Insolvenz behalten können. Fan und White (2003) schätzen, dass Eigenheimbesitzer in Staaten mit hohen oder unbegrenzten Befreiungen eine um 35\% höhere Wahrscheinlichkeit haben, ein Unternehmen zu gründen, als in Staaten ohne oder mit sehr geringen Befreiungen. Zu ähnlichen Ergebnissen kommen Armour und Cumming (2008) für 15 Länder in Nordamerika und Europa.

Ein schwaches Insolvenzrecht bietet den Schuldnern mehr Sicherheit und steigert die Risikobereitschaft. Andererseits reduzieren hohe Befreiungen die Verwertungsrate bei Insolvenzen und verursachen den Banken und Investoren hohe Verluste. Diese müssen jedoch das so gesteigerte Risiko kompensieren und höhere Zinsen verlangen. Kleinere Unternehmen und Privathaushalte zahlen daher in Staaten mit hohen Befreiungen entsprechend höhere Zinsen und erhalten öfter keinen Kredit als in anderen Staaten mit geringen Befreiungen. Höhere Befreiungen stärken eben die Stellung der Schuldner auf Kosten der Gläubiger und stehen für einen schwachen Gläubigerschutz. La Porta u.a. (2013) stellen große Unterschiede zwischen den Staaten im Gläubigerschutz und damit im Kreditzugang fest. Sie messen die Stellung der Gläubigerrechte und die damit verbundene Verwertungsrate im Konkursverfahren. Demnach geht eine Verbesserung der Gläubigerrechte von einem Land zum anderen (um zwei Standardabweichungen) mit einem um 15 Prozentpunkte höheren Anteil von Privatkrediten am BIP einher. Die Verwertungsrate bei notleidenden Krediten hängt jedoch nicht nur von den gesetzlichen Regelungen ab, sondern auch von der effektiven Rechtsdurchsetzung in den Konkursverfahren. Nach La Porta u.a. (2013) geht eine Verbesserung der Effizienz in der Schuldenbetreibung (um zwei Standardabweichungen) mit einem um 27 Prozentpunkte höheren BIP-Anteil von Privatkrediten einher. Abbildung 31 zeigt für Österreich im EU-Vergleich eine überdurchschnittlich gute Verwertungsrate aus dem Insolvenzvermögen, was auf einen relativ gut ausgebauten Gläubigerschutz schließen lässt.

Die Regeln des Insolvenzrechts spiegeln eine schwierige Abwägung zwischen Gläubigerschutz und den beruflichen Chancen der Schuldner wider. Indem nur das vorhandene Finanz- und Sachvermögen verwertet wird, während zukünftiges Einkommen frei bleibt, kann das Insolvenzrecht den Neustart erleichtern. Gerade wenn es gilt, die Anzahl von Unternehmensgründungen zu steigern, dann kann der zweite Versuch eine ergiebige Quelle dafür sein. Selbst das Scheitern im ersten Versuch kann wichtige Erfahrungen generieren, so dass ein zweiter Anlauf eine mindestens so hohe Erfolgswahrscheinlichkeit hat wie eine erstmalige Unternehmensgründung. Gompers u.a. (2010) untersuchen Daten zu Unternehmensgründungen in den U.S.A., wovon ungefähr 10\% auf Mehrfachgründer entfallen. Demnach hat ein wagnisfinanzierter Unternehmer, der schon bei seiner ersten Gründung das Unternehmen an der Börse einführen konnte, eine Wahrscheinlichkeit von $30 \%$, auch seine nächste Gründung erfolgreich zu platzieren. Demgegenüber hat ein erstmaliger Gründer nur eine Erfolgswahrscheinlichkeit von 21\%, und ein vorher gescheiterter Unternehmer eine Chance von 22\%. Auch nach einem ersten Fehlschlag haben Mehrfachgründer keine schlechteren Erfolgsaussichten als andere erstmalige Gründer. Die 
Autoren betonen, dass Mehrfachgründer sich von vornherein durch besondere unternehmerische Fähigkeiten auszeichnen und eine positive Auswahl darstellen. Sie sind bereits bei ihrem ersten Versuch wesentlich erfolgreicher als andere Unternehmer, die nur einen einzigen Start-up gründen. Dazu kommt, dass bei Kapitalgebern, Beschäftigten und Kunden nichts so überzeugend ist wie Erfolg. Gründer, die im ersten Versuch erfolgreich waren, haben es bei der nächsten Gründung leichter, Kapital und Personal anzuwerben und Kunden zu überzeugen, was ihre Erfolgsaussichten noch einmal steigert.

\section{Abbildung 31: Verwertungsrate in den Konkursverfahren, 2016}

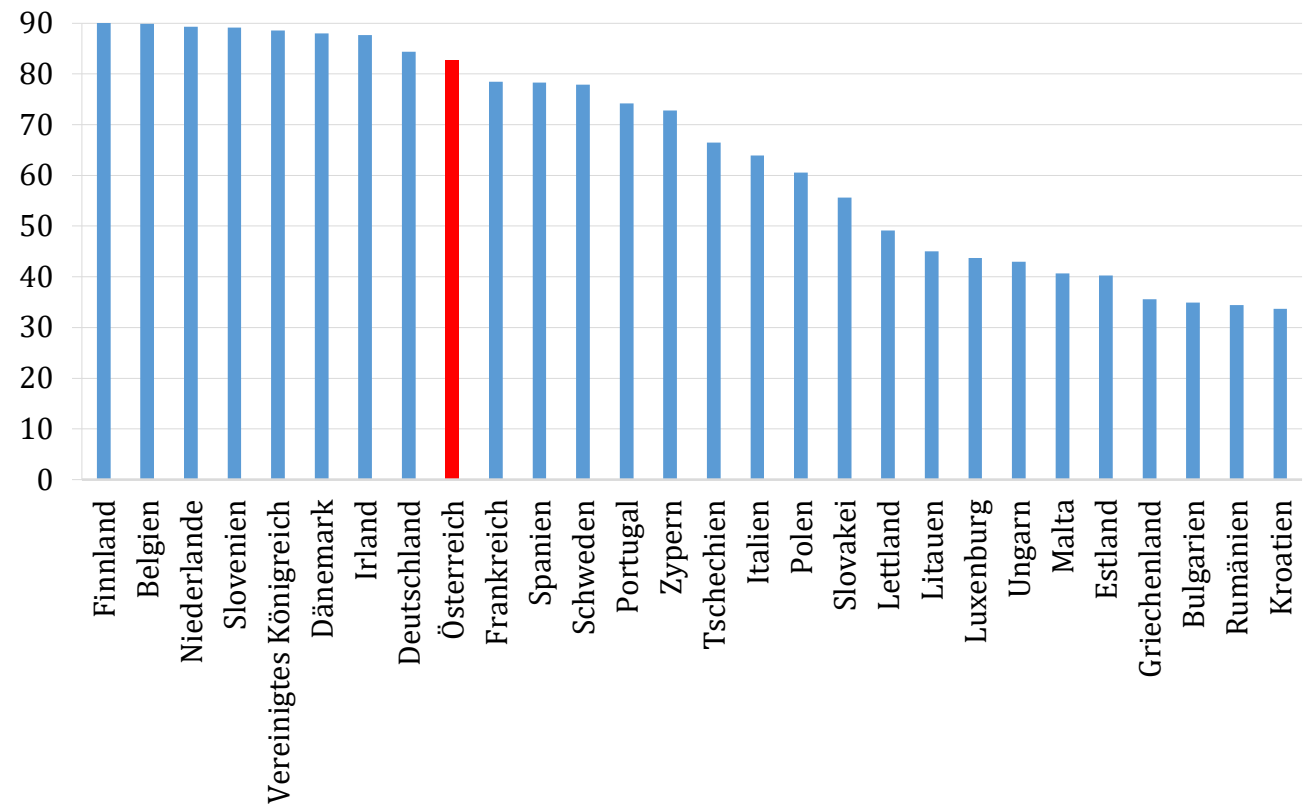

Quelle: www.doingbusiness.org/data/exploretopics/resolving-insolvency, abgerufen am 2. 3. 2017, Doing Business - World Bank.

Ein klug austariertes Insolvenzrecht unterstützt Innovation und Strukturwandel, damit das Kapital dort zum Einsatz kommt, wo es am meisten zum Einkommen und zum Fortschritt beitragen kann. Die Diskussion macht jedoch deutlich, dass das Insolvenzrecht viele Abwägungen treffen muss, für die es keine eindeutige Lösung gibt. Ein Konsens der Forschung ist, dass im Hinblick auf die Erleichterung zum Neustart das künftige Arbeitseinkommen geschützt werden soll, während die Freibeträge für das private Finanz- und Sachvermögen im Hinblick auf einen guten Gläubiger- und Investorenschutz eher gering bleiben sollen. Der Schutz des Humankapitals erleichtert den Neustart für eine zweite Unternehmensgründung mit mehr Erfahrung. Ein geringer Freibetrag für das geschützte Vermögen verbessert den Investorenschutz und damit die Verfügbarkeit von günstigerer Kreditfinanzierung und Risikokapital. Eine innovative Wirtschaft braucht risikobereite Unternehmer (Neustart) und mehr Risikokapital (strenger Gläubigerschutz) gleichermaßen. In Österreich gibt es im Insolvenzrecht grundsätzlich keine Freibeträge im Finanz- und Sachvermögen, was den Gläubigerschutz und damit die Verfügbarkeit von neuer Finanzierung stärkt. Allerdings können Arbeitseinkommen in der Privatinsolvenz bis zu 7 Jahre herangezogen werden. Eine 
Verkürzung und Einschränkung dieser Regelung würde die Neigung zu risikobehafteten Unternehmensgründungen und den Neustart erleichtern.

\subsection{Wettbewerbspolitik und Strukturwandel}

Innovation hängt eng mit Wettbewerb und Strukturwandel zusammen. Abbildung 32 veranschaulicht den beidseitigen Zusammenhang. Wettbewerb zwingt die Unternehmen, sich von der Konkurrenz abzuheben. Erfolgreiche Innovation schafft den Qualitäts- und Kostenvorsprung, mit dem sie am Markt höhere Gewinnspannen erzielen können. Eine einmal errungene marktbeherrschende Position und die damit möglichen Gewinne locken jedoch neue Konkurrenten an. Wenn ein Unternehmen seine F\&E-Aktivitäten und damit die Erneuerung seines Angebots vernachlässigt, büßt es rasch Marktanteile ein und wird von der Konkurrenz verdrängt. Innovation führt daher zu permanentem Strukturwandel und zu einer Umlenkung von Arbeit und Kapital. Große Konzerne treiben Strukturwandel und Faktorreallokation innerhalb der Gruppe an, indem sie reife Produkte und Verfahren durch neue und profitablere ersetzen und im Zuge dieses Produktzyklus Arbeit und Kapital von alten auf neue Verwendungen umlenken. Gleichzeitig scheiden träge Anbieter aus dem Markt aus oder werden von leistungsfähigeren Konkurrenten übernommen, und neue Start-ups kommen dazu.

Abbildung 32: Wettbewerb, Innovation und Strukturwandel

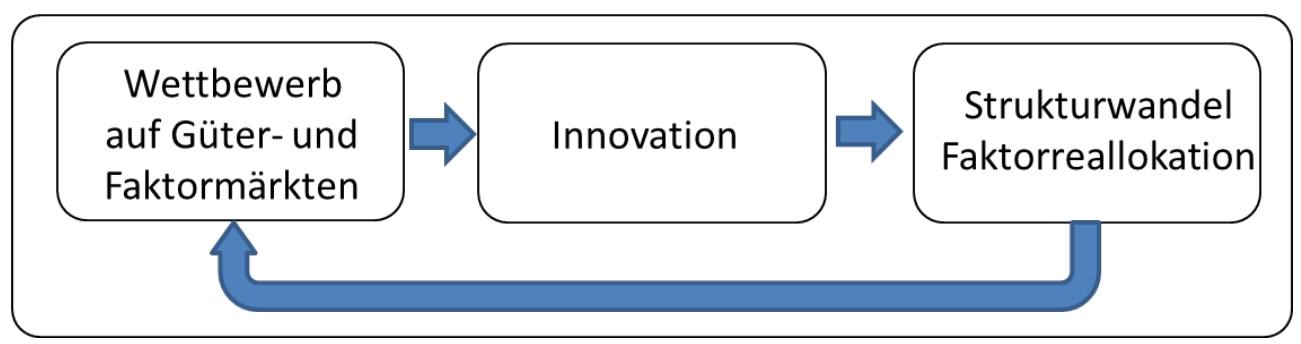

Quelle: eigene Darstellung

Marktzutritt und Austritt wälzen die Unternehmenslandschaft laufend um und begünstigen eine Auswahl der Besten. In Österreich gab es im Jahr 2014 etwa 242.165 Arbeitgeberunternehmen (d.h. Unternehmen mit mindestens einem unselbständig Beschäftigten, Statistik Austria, Arbeitgeberunternehmensdemografie ab 2015). Im Durchschnitt der Jahre 2012-2014 haben die Neugründungsrate 8,9\% und die Schließungsrate 8,2\% betragen.

Arbeit und Kapital müssen von schrumpfenden zu wachsenden Unternehmen fließen. Erst indem sie sich von überkommenen und unrentablen Verwendungen lösen und sich zu profitablen und wachsenden Unternehmen hinwenden, können sie von Innovation

profitieren. Produktivität und Einkommen nehmen erst zu, wenn Arbeit und Kapital dorthin fließen, wo ihr Einsatz überdurchschnittlich rentabel ist, anstatt in alten Verwendungen zu verharren, wo nur wenig Wertschöpfung möglich ist und die Jobs unsicher sind. 


\subsubsection{Wettbewerb und Innovation}

Wettbewerb steigert die Leistungsfähigkeit der Unternehmen, begünstigt neue Entdeckungen und erhöht die Produktivität (Romer, 2007). Allerdings kommt es auf die richtige Natur des Wettbewerbs an. Ohne Aussicht auf Gewinne durch eine beschränkte Marktmacht wäre Innovation nicht möglich. Die Unternehmen könnten keinen Ertrag auf die vorausgehenden F\&E-Investitionen erzielen und die F\&E-Kosten nicht erwirtschaften. Mit einem Qualitätsund Kostenvorsprung können sie jedoch einen Preisaufschlag gegenüber dem nächst besten Konkurrenten durchsetzen und eine Gewinnspanne erwirtschaften, um die F\&E-Kosten gerade noch abzudecken. Entscheidend ist, dass ein Markt „bestreitbar“ bleibt, d.h., dass jederzeit ein Marktzutritt von neuen Konkurrenten möglich ist. Diese potentielle Konkurrenz zwingt die etablierten Unternehmen, den Preisaufschlag auf einen Betrag zu begrenzen, der gerade dem Qualitäts- und Kostenvorsprung entspricht. Wird jedoch der Marktzutritt behindert, entsteht mehr Marktmacht für die etablierten Anbieter. Sie können Preisaufschläge durchsetzen, die den Qualitäts- und Kostenvorsprung gegenüber neuen Anbietern übersteigen und den Unternehmen mehr Gewinne zukommen lassen, als zur Abdeckung der F\&E-Investitionen notwendig ist. Sie können mehr Kaufkraft der Nachfrager abschöpfen, als tatsächlich durch das bessere Preis-Leistungs-Verhältnis gerechtfertigt wäre. Solche übernormalen Gewinne steigern den Innovationsanreiz über das notwendige Ausmaß hinaus, aber die Innovationen dienen nur mehr den Unternehmen und nicht mehr den Konsumenten, bei denen zu viel Kaufkraft abgeschöpft wird.

Es kann also ein zu viel und ein zu wenig an Wettbewerb geben (vgl. De Bondt und Vandekerckhobe, 2012, zu einem Forschungsüberblick zum Verhältnis von Wettbewerbsund Innovationsintensität). Nach Shapiro (2012) hat sich folgender Konsens herausgebildet: (1) Die Aneignung der Innovationserträge durch das innovierende Unternehmen stärkt den Innovationsanreiz. (2) Damit die Märkte dynamisch effizient sind, müssen sie „bestreitbar“ sein. Innovationen können nur dann im richtigen Ausmaß erfolgen, wenn die Unternehmen sich tatsächlich alle F\&E-Erträge aneignen können, damit alle gesellschaftlich relevanten Kosten und Erträge in die private F\&E-Entscheidung einfließen können. Deshalb spielt der Patentschutz eine große Rolle, der den Unternehmen die Erträge in Form eines exklusiven Verwertungsrechts ihrer Erfindung über eine beschränkte Zeit zusichert (vgl. Abschnitt 4.2). Nach Ablauf des Patentschutzes wird der Markt „bestreitbar" und das Unternehmen ist wieder dem Wettbewerb ausgesetzt. Die Dauer des Patentschutzes muss die richtige Abwägung treffen zwischen der Aneignung von Innovationserträgen und der Verhinderung von Marktmissbrauch. Im Ergebnis sollen die Gewinne aus der privaten Verwertung der Erfindung gerade ausreichen, um die vorausgehenden F\&E-Kosten abzudecken, ohne dass Netto ein überschüssiger Barwert von Gewinnen entstünde.

Was in statischer Sicht als Ausnutzung von Marktmacht erscheint, ist in dynamischer Betrachtung ein vollständiger Wettbewerb mit freiem Marktzutritt bei Innovationen („Bestreitbarkeit"), der einen Nettogewinn über den gesamten Investitionshorizont ganz beseitigt. Solange also die Wettbewerbspolitik die Aneignung von Innovationserträgen sicherstellt und die Bestreitbarkeit durch Reduktion von Markteintrittsbarrieren fördert, kann sie die richtige Innovationsintensität erreichen (vgl. European Commission, 2016, und die Positionen von Schumpeter, 1942, und Arrow, 1962). Aghion u.a. (2005) verorten mit ihren ökonometrischen Schätzungen die maximale Innovationsintensität bei einem 
Wettbewerbsgrad nahe der vollständigen Konkurrenz. Im Einklang damit argumentieren Aghion u.a. (2011), dass industriepolitische Interventionen die technologische Leistungsfähigkeit einer Branche umso wirkungsvoller steigern können, je höher der Wettbewerbsgrad in diesem Sektor ist.

Empirische Studien zeigen vorwiegend positive Effekte der Wettbewerbsintensität auf die Produktivität von Unternehmen (Holmes und Schmitz, 2010; Syverson, 2011):

(1) Nickel (1996) zeigt für Großbritannien, dass ein intensiverer Wettbewerb das Wachstum der Faktorproduktivität beschleunigt. Die geringere Wettbewerbsintensität im Sektor „Electrical Engineering" hätte das Produktivitätswachstum um 2,4 Prozentpunkte unter den Durchschnitt gedrückt, während sie in „Mechanical Engineering“ mit höherer Wettbewerbsintensität einen Prozentpunkt über dem Durchschnitt lag.

(2) Buccirossi u.a. (2013) analysieren für 22 Branchen in 12 OECD-Staaten zwischen 19952005, wie die Wettbewerbspolitik das Wachstum der Multifaktorproduktivität beeinflusst. Sie stellen einen positiven und signifikanten Effekt der Wettbewerbspolitik fest. Verschärfungen der Wettbewerbspolitik hätten anfangs der 2000er Jahre zu einem Produktivitätswachstum von $22 \%$ im britischen Nahrungsmittelsektor beigetragen.

(3) Griffith u.a. (2010) untersuchen, wie die Verschärfung des Wettbewerbs durch das Binnenmarktprogramm auf die Innovationsintensität europäischer Unternehmen wirkte. Ihre Daten umfassen neun Mitgliedsstaaten und 12 Industriesektoren von 1987 bis 2000. Die Binnenmarktreformen hätten die F\&E-Intensität des „Metal products sector“ um 1,2 Prozentpunkte gesteigert und damit das Produktivitätswachstum um 0,7 Prozentpunkte beschleunigt, was einem Drittel des gesamten Anstiegs der Wachstumsrate entspricht.

In Österreich wird die Bedeutung des Wettbewerbs oft unterschätzt. Der hohe Anteil der verstaatlichten Industrie, korporatistische Übereinkünfte und das hochregulierte Gewerbe prägten lange Zeit die Entwicklung. Der EU-Beitritt hat jedoch viele Verbesserungen bewirkt. Allerdings erscheint die Wettbewerbsbehörde nach wie vor als relativ schwach und ist unterausgestattet (Böheim, 2010). Die OECD (Alemani u.a., 2013) hat Indikatoren entwickelt, um die wettbewerbliche Orientierung zu vergleichen. Tabelle 14 zeigt vier Indikatoren, wobei 0 die stärkste und 6 die schwächste Orientierung am Wettbewerbsprinzip darstellt.

Nach dem OECD-Index zählt Österreich bei der Aktionsreichweite der Wettbewerbsbehörde sowie bei der Bekämpfung von wettbewerbsschädlichem Verhalten sogar zu den Staaten mit der stärksten Wettbewerbsorientierung. Ein Mangel besteht bei der Qualität und Transparenz in der Durchsetzung des Wettbewerbsrechts. Auch der Einsatz der Wettbewerbsbehörde für die Stärkung des Wettbewerbs in Österreich liegt hinter jenem von Staaten wie Dänemark oder den Niederlanden. Abgesehen von der Durchsetzung des Wettbewerbsrechts scheint Österreich nach Tabelle 14 aber nicht signifikant weniger wettbewerbsorientiert als andere Länder zu sein. Neben der Wettbewerbspolitik wird die Intensität des Wettbewerbs vor allem durch die geltenden Marktregulierungen auf den Güterund Faktormärkten beeinflusst. Eine geringere Regulierungsdichte geht mit einer höheren Wettbewerbsintensität einher, wenn zugleich eine starke Wettbewerbspolitik existiert, und begünstigt damit ein stärkeres Produktivitätswachstum. 


\begin{tabular}{llllc}
\hline & Aktionsreichweite & $\begin{array}{l}\text { Bekämpfung von } \\
\text { wettbewerbsschäd- } \\
\text { lichem Verhalten }\end{array}$ & $\begin{array}{l}\text { Qualität und } \\
\text { Transparenz bei der } \\
\text { Durchsetzung des } \\
\text { Wettbewerbsrechts }\end{array}$ & $\begin{array}{c}\text { Einsatz der } \\
\text { Wettbewerbsbehörde } \\
\text { für eine Stärkung des } \\
\text { Wettbewerbs }\end{array}$ \\
\hline Österreich & 0,14 & 0,00 & 0,90 & 0,86 \\
Dänemark & 0,29 & 0,00 & 0,00 & 0,21 \\
Finnland & 0,29 & 0,16 & 0,15 & 0,64 \\
Deutschland & 0,00 & 0,00 & 0,30 & 0,86 \\
Niederlande & 0,29 & 0,32 & 0,15 & 0,64 \\
Schweden & 0,29 & 0,00 & 0,60 & 0,86 \\
Schweiz & 0,29 & 0,00 & 1,20 & 0,43 \\
\hline
\end{tabular}

Anmerkung: Der Index liegt zwischen 0-6, wobei 0 die stärkste Durchsetzung bzw. Orientierung am Wettbewerbsprinzip anzeigt und 6 die schwächste.

Quelle: Alemani u.a. (2013)

Arnold u.a. (2011) zeigen empirisch, dass in schwach regulierten Branchen der Anteil der Unternehmen mit hohem Produktivitätswachstum und auch der Median der Produktivität höher ist als bei hohem Regulierungsgrad. In Abbildung 33 sind die Länder nach dem Wert für 2013 in absteigender Reihenfolge geordnet.

Abbildung 33: Regulierungsdichte der Produktmärkte (1998-2013)

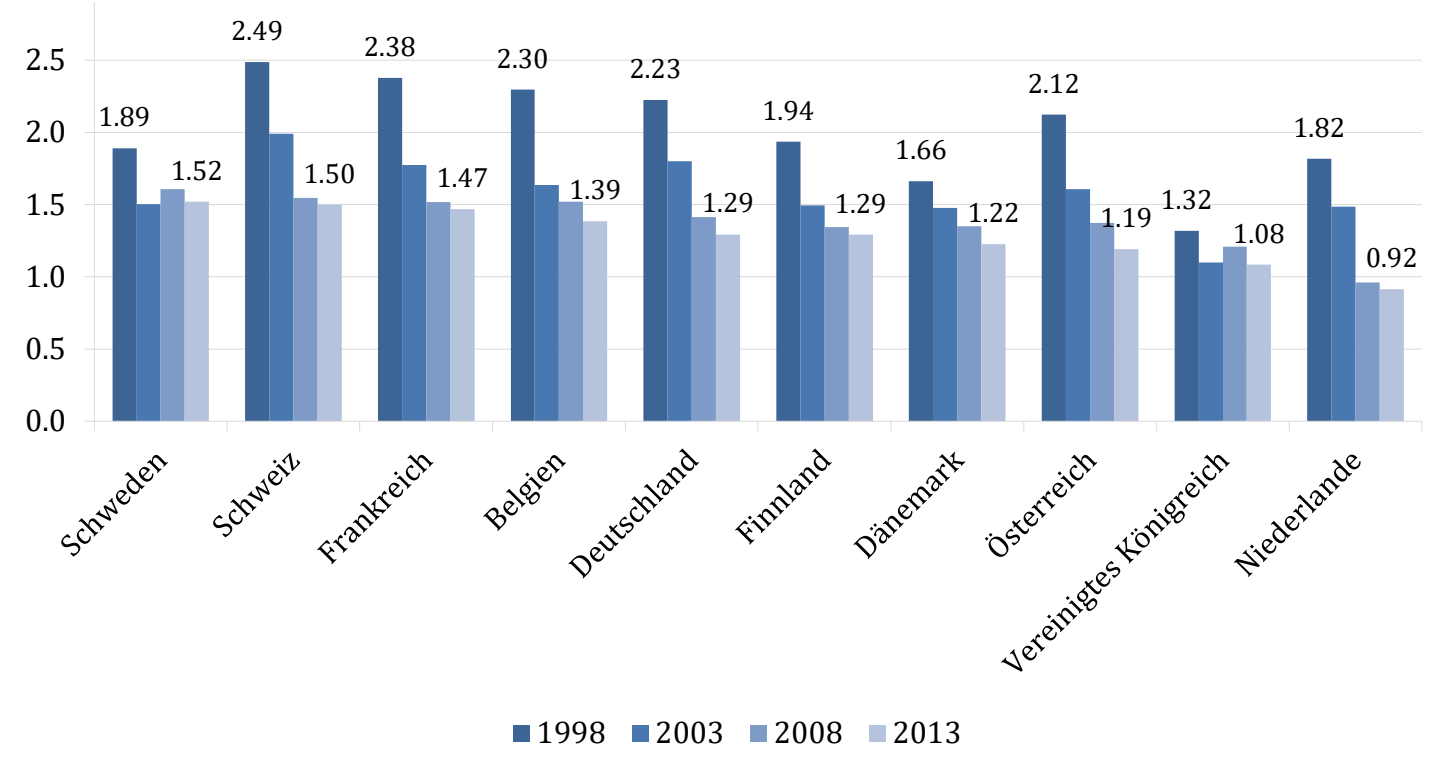

Bemerkung: 0 ist die geringste und 6 die stärkste Wettbewerbseinschränkung durch Regulierung. Der Indikator der OECD folgt aus einer Aggregation von 18 Teilindikatoren wie z.B. Umfang staatlich kontrollierter Unternehmen, Einmischung des Staates in Netzwerkindustrien, Existenz von Preiskontrollen, administrative Belastungen, oder rechtliche Eintrittsbarrieren für Unternehmen. Quelle: Koske (2015) 
Demnach zählt Österreich nach den Niederlanden und Großbritannien zu den Ländern mit relativ liberaler Produktmarktregulierung. Österreich hat seit dem EU-Beitritt deutliche Liberalisierungsschritte gesetzt und liegt nun vor Staaten wie Schweden oder der Schweiz.

Marktmacht erlaubt den Unternehmen, höhere Preise durchzusetzen. Je höher die Preis- und Gewinnaufschläge sind, desto geringer ist im Allgemeinen die Wettbewerbsintensität. Tabelle 15 erlaubt daher eine direkte Beschreibung der Wettbewerbsintensität verschiedener Branchen und Länder.

Tabelle 15: Durchschnittliche Gewinnaufschläge 1981-2004 nach Branchen

\begin{tabular}{lccc}
\hline & Sachgütererzeugung und Bauwesen & Dienstleistungen & Insgesamt \\
\hline Frankreich & 1,15 & 1,26 & 1,21 \\
Niederlande & 1,13 & 1,31 & 1,22 \\
Belgien & 1,14 & 1,29 & 1,22 \\
Spanien & 1,18 & 1,37 & 1,26 \\
Finnland & 1,22 & 1,39 & 1,28 \\
Österreich & 1,2 & 1,45 & 1,32 \\
USA & 1,28 & 1,36 & 1,33 \\
Deutschland & 1,16 & 1,54 & 1,37 \\
Eurozone & 1,18 & 1,56 & 1,61 \\
Italien & 1,23 & 1,87 & 1,3 \\
\hline
\end{tabular}

Bemerkung: Die Eurozone umfasst alle angeführten Staaten mit Ausnahme der USA.

Quelle: Christopoulou und Vermeulen (2012), Tabelle 1.

In allen Staaten sind die Preisaufschläge im stärker regulierten Dienstleistungssektor höher als in der Industrie, die wesentlich stärker der internationalen Konkurrenz ausgesetzt ist. Das zeigt einmal mehr, dass eine höhere Regulierung die Wettbewerbsintensität schwächt, während beidseitiger Marktzugang den Wettbewerb verschärft, die Preise drückt und damit die Kaufkraft der Nachfrager stärkt. Im Vergleich zu den Innovationsführern Finnland und Niederlande sind die Gewinnaufschläge in Österreich höher, allerdings liegen sie niedriger als im EU-Durchschnitt und in Deutschland. Die Wettbewerbsintensität kann also in Österreich im internationalen Vergleich als durchaus gut gewertet werden und dürfte kein besonderes Innovationshemmnis darstellen.

\subsubsection{Innovation und Strukturwandel}

Innovation bedeutet: Neues ersetzt Altes. Erfolgreiche Innovation ist der Schlüssel für das Unternehmenswachstum. Innovative Wachstumsunternehmen erobern Marktanteile oder schaffen völlig neue Märkte, die Nachfrage von anderen Märkten abziehen. Weniger innovative Unternehmen können angesichts besserer Leistungen der Konkurrenz bei den Kunden nicht mehr punkten, verlieren Marktanteile, müssen schrumpfen oder scheiden ganz aus. Beschäftigung und Investitionen folgen der Entwicklung der Unternehmen. Marktzutritt und Austritt erneuern die Unternehmenslandschaft und führen dazu, dass Arbeit und Kapital dorthin wandern, wo mehr Wertschöpfung und Wachstum möglich sind. Indem weniger produktive durch produktive Unternehmen ersetzt werden, steigt die durchschnittliche 
Produktivität. Auch die großen, multinationalen Konzerne sind keine unsinkbaren Schiffe. Sie können nur mit andauernder Innovation ihre Stellung halten, indem sie im Produktzyklus ihr Angebot ständig erneuern, also innovative und profitable Produkte und Anwendungen ausbauen und ein obsoletes Angebot vom Markt nehmen und Produktionskapazitäten stilllegen. Um ihre überdurchschnittliche Produktivität zu erhalten, müssen sie auf internen Arbeits- und Kapitalmärkten Belegschaft und Investitionskapital ständig neu einsetzen. Ohne Strukturwandel und nachfolgende Faktorreallokation können Innovationen sich nicht durchsetzen und damit Produktivität, Beschäftigung und Einkommen steigern (Keuschnigg u.a., 2014).

Der Strukturwandel durch Innovation und die nachfolgende Umlenkung von Arbeit und Kapital von altem zu neuem Einsatz ist in der empirischen Forschung eindrücklich dokumentiert und trägt zum größten Teil des Produktivitätswachstums in der Wirtschaft bei. Wettbewerbliche und flexible Güter-, Arbeits- und Kapitalmärkte sind entscheidend. Je erfolgreicher eine Marktwirtschaft diesen Wandel bewältigt, desto mehr Produktivität und Wachstum kann ein Land aus F\&E-Investitionen herausholen. In manchen Branchen ist etwa die Hälfte des Produktivitätswachstums auf die Verschiebung von Marktanteilen und der dafür notwendigen Wanderung von Arbeit und Kapital zwischen erfolgreichen und weniger erfolgreichen Unternehmen zurückzuführen (Haltiwanger, 2011). Auch große Konzerne wie z.B. die VOEST, Toyota und Siemens müssen stets ihre Angebotspalette erneuern und ihre Produktivität steigern, damit sie angesichts der laufenden Herausforderung durch die Konkurrenz wettbewerbsfähig bleiben.

Tabelle 16: Faktorreallokation zwischen innovativen und nicht innovativen Unternehmen

\begin{tabular}{lccc}
\hline & $\begin{array}{c}\text { Physisches Kapital } \\
\text { (Nettoinvestitionsrate }\end{array}$ & $\begin{array}{c}\text { Arbeit } \\
\text { (Aufnahmerate) }\end{array}$ & $\begin{array}{c}\text { Finanzkapital } \\
\text { (Nettozuflüsse) }\end{array}$ \\
\hline $\begin{array}{l}\text { Innovative Unternehmen } \\
\text { Nicht innovative Unternehmen }\end{array}$ & $+0,4 \%$ bis $+0,9 \%$ & $-0,1 \%$ bis $0,7 \%$ & $+0,6 \%$ bis $0,7 \%$ \\
$\begin{array}{l}\text { Medianwerte gesamte } \\
\text { Stichprobe }\end{array}$ & $-1,2 \%$ bis $-1,6 \%$ & $-1 \%$ bis $-3 \%$ & $-0,1 \%$ bis $-0,6 \%$ \\
\hline
\end{tabular}

Quelle: Kogan u.a. (2012).

Tabelle 16 illustriert mit Daten aus den USA, wie die Innovation den Strukturwandel befördert und Arbeit und Kapital von nicht innovativen und wenig profitablen Unternehmen $\mathrm{zu}$ innovativen und stark expandierenden Firmen umgelenkt werden. Die Nettoinvestitionsrate für Sachkapital beträgt im Durchschnitt 10\%. Die innovativen Unternehmen investieren um 0,4-0,9 Prozentpunkte mehr, und nicht innovative Firmen um 1,2-1,6 Prozentpunkte weniger als der Durchschnitt. Während ein durchschnittliches Unternehmen die Beschäftigung um 3\% ausdehnt, wächst die Beschäftigung bei innovativen Firmen deutlich rascher $(+2,9$ bis $+3,7 \%)$, aber bei nicht innovativen Firmen nur unwesentlich ( 0 bis $+2 \%$ ). Auch das Finanzkapital fließt von nicht innovativen zu innovativen Unternehmen.

Die Faktorreallokation erfolgt durch Umschichtung „innerhalb“ großer Konzerne und durch Wanderung „zwischen“ Unternehmen und beeinflusst auf beiden Wegen das Produktivitätswachstum (Syverson, 2011). Eine Studie von Lentz und Mortensen (2008) zur 
industriellen Dynamik in Dänemark liefert ein eindrückliches Bild davon. Demnach sind ganze 75\% des Produktivitätswachstums von 1992 bis 1997 durch die Umlenkung der Produktionsfaktoren hin zu innovativen Verwendungen zustande gekommen. Von diesen Reallokationsgewinnen gehen ein Drittel auf Marktzutritte und Austritte und zwei Drittel auf den Neueinsatz von Ressourcen innerhalb bestehender Unternehmen zurück. Für die USA zeigen Foster u.a. (2001), dass von 1995 bis 1999 das Produktivitätswachstum im Einzelhandel zu 98\% durch den Eintritt von produktiven und den Austritt von weniger produktiven Unternehmen verursacht wurde. Haltiwanger (2011) spaltet das durchschnittliche Wachstum der Multifaktorproduktivität in der US-Industrie über den Zeitraum 1977-1997 in seine Komponenten auf. Nach Abbildung 34 betrug das Produktivitätswachstum insgesamt 5,13\%, wovon 3,44 Prozentpunkte auf Produktivitätszuwächse innerhalb bestehender Firmen und 1,7 Prozentpunkte auf die Reallokation zwischen Unternehmen entfielen. Davon wiederum entfällt mit 1,35\% der größte Teil auf den Nettozutritt neuer, überdurchschnittlich produktiver Unternehmen und nur 0,35\% auf den Ersatz von Marktaustritten durch ebenso viele Zutritte.

Abbildung 34: Beiträge zum Produktivitätswachstum der US-Industrie, 1977-1997

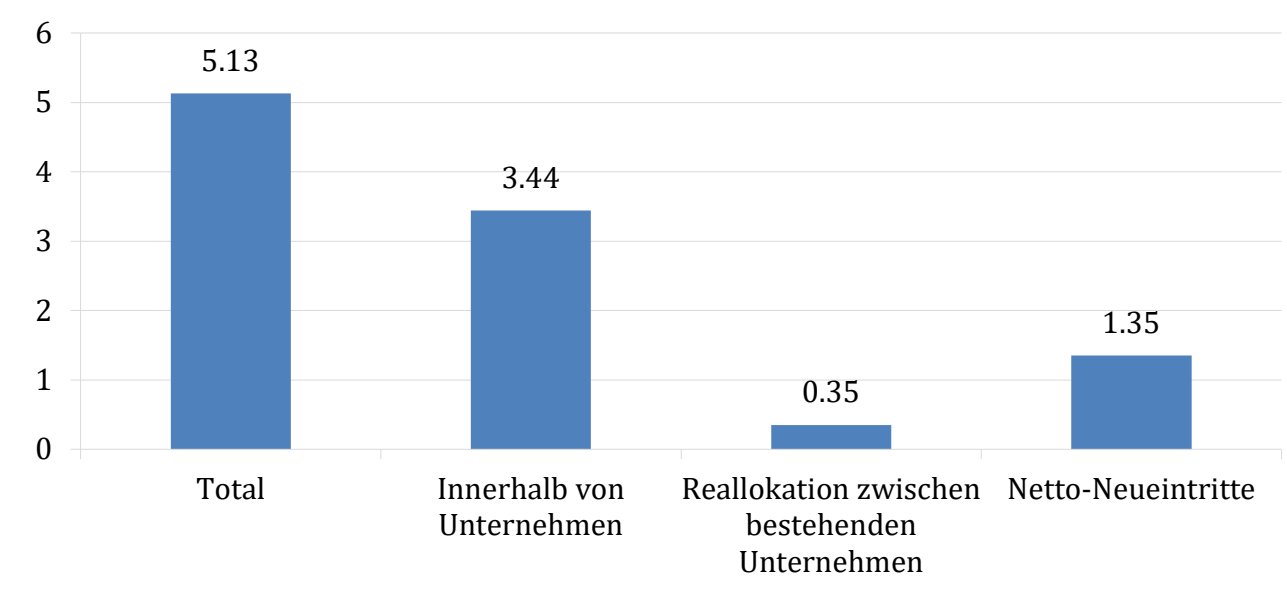

Bemerkung: Wachstumsrate Multifaktorproduktivität in 5-Jahres-Schritten, ausgewählte Branchen Quelle: Haltiwanger (2011)

Eine Aufspaltung der Produktivitätsentwicklung in der österreichischen Industrie für den Zeitraum von 2002-2007 unterstreicht ebenfalls die große Bedeutung der Reallokation. Die Studie von Hölzl und Lang (2011) ist allerdings nicht ganz vergleichbar, weil sie nicht die Faktor-, sondern die Umsatzproduktivität betrachtet, zwischen exportierenden und nicht exportierenden Unternehmen unterscheidet und eine etwas andere Aufspaltung ausweist. Exportfirmen sind typischer Weise produktiver und F\&E-intensiver. In Tabelle 17 ist daher wenig überraschend das Wachstum der Umsatzproduktivität bei Exportfirmen mit 21,5\% über die gesamte Periode deutlich höher als bei nicht exportierenden Unternehmen (12,3\%). Schon in dieser unterschiedlichen Expansion ist ein Reallokationseffekt enthalten, der hier nicht berücksichtigt ist. Bei Exportfirmen entfielen $78 \%$ des gesamten Produktivitätswachstums (21,5\%) auf Produktivitätsgewinne innerhalb bestehender Unternehmen (16,8\%). Entsprechend geringer fiel der Beitrag der Reallokationskomponente 
(Anteil 22\%) aus, wobei mit 4,5\% der Großteil der Reallokation innerhalb der Unternehmensgrenzen erfolgte, während Marktzutritte und Austritte mit 0,2\% nur einen verschwindend kleinen Teil beisteuerten. Bei dem überdurchschnittlich hohen Wachstum der innovativen Exportfirmen spielt eben der Marktaustritt eine weniger bedeutende Rolle. Bei den weniger rasch wachsenden Nicht-Exportfirmen machte dagegen die Wachstumskomponente innerhalb der Unternehmen nur $45 \%$ des gesamten Produktivitätswachstums aus, während ein wesentlich größerer Teil auf Zutritte und Austritte zurückzuführen war.

Tabelle 17: Zerlegung der Umsatzproduktivität für die österreichische Industrie, 2002-2007

\begin{tabular}{lcccc}
\hline & $\begin{array}{c}\text { Gesamte } \\
\text { Veränderung }\end{array}$ & \multicolumn{2}{c}{ Ständige Unternehmen } & Marktzutritt \\
und Austritt
\end{tabular}

Anmerkung: Veränderungen in Prozent; Daten: Statistik Austria, Konjunkturstatistik; WKO, WIFO Quelle: Hölzl und Lang (2011)

Wie können Reallokation und Strukturwandel erleichtert werden, damit Innovation sich besser durchsetzt? Dazu ist es zuerst notwendig, die Barrieren für einen Marktzutritt innovativer Unternehmen abzubauen und die Kosten für den Marktaustritt zu reduzieren. Produktmarktregulierungen dienen zwar auch der Konsumentensicherheit, dienen aber allzu oft dem Schutz vor Wettbewerb und laufen den Interessen der Konsumenten sogar zuwider. Die hohen Regulierungskosten wiegen gerade für innovative junge Unternehmen schwer und steigern die Markteintrittskosten, welche den Strukturwandel erschweren und das Produktivitätswachstum hemmen. Poschke (2010) schätzte, dass ein Anstieg der Eintrittskosten vom niedrigen dänischen Niveau auf ein mittleres Niveau wie etwa in Spanien das BIP pro Kopf längerfristig um 7,5 bis 10\% senken würde. Weniger Unternehmen im Markt bedeuten zunächst geringeren Wettbewerb, höhere Preise und niedrigeren Output. Im Laufe der Zeit erhöhen sich die Überlebenschancen von weniger produktiven Unternehmen, die den produktiveren Firmen Marktanteile stehlen und ihre Expansion bremsen. Das durchschnittliche Produktivitätswachstum wird langsamer. Zudem ist die Substituierbarkeit von Produkten geringer, wenn es nur wenige Angebote gibt, und die Kundenbindung an die existierenden Anbieter größer. Das erschwert den Eintritt und das Wachstum neuer Konkurrenten, weil es schwieriger wird, Kunden von den existierenden Unternehmen abzuwerben. F\&E-Investitionen werden weniger rentabel und die Anreize für die Einführung neuer Technologien sinken (Poschke, 2010). Diese langfristigen Produktivitätseffekte multiplizieren die statischen Realeinkommensverluste von Wettbewerbsbeschränkungen und führen zu den stark negativen langfristigen Auswirkungen von Zutrittsbarrieren.

Auch Marktaustrittsbarrieren beeinflussen die Innovationsanreize ungünstig. Hohe Austrittskosten, insbesondere hoher Kündigungsschutz, reduzieren die Bereitschaft zu riskanten F\&E-Investitionen, weil bei einem Scheitern die Kosten höher sind. Die Einführung von innovativen IKT-Technologien erhöht die Volatilität und damit das Risiko für die 
Unternehmen. Van Ark u.a. (2008) und Bartelsman (2013) zeigen, dass IKT-intensive Sektoren kleiner sind in Staaten, die über einen rigoroseren Kündigungsschutz verfügen. Damit erklären die ForscherInnen die raschere Diffusion von IKT-Technologien in den USA.

Handelsbarrieren haben über die Regulierung der Importkonkurrenz einen starken Einfluss auf die Wettbewerbsintensität und in der Folge auf Innovation und Strukturwandel im Inland. Der Zugang zu preisgünstigen Importprodukten guter Qualität stärkt das Realeinkommen und damit den Wohlstand der Konsumenten, solange in der Produktion eine günstige Spezialisierung der heimischen Wirtschaft gelingt. Wer im Status Quo verhaftet ist, für den wird die Importkonkurrenz zur Gefahr. Wenn man offen für Wandel ist und neue Beschäftigung in anderen wachsenden Branchen und Unternehmen mit Exportvorteilen sieht, dann ist die Spezialisierung im Welthandel und der damit verbundene Zugang zu günstigen Produkten eine Chance für mehr Wohlstand.

Bloom u.a. (2011) untersuchen die Auswirkungen von Billigimporten aus China in zwölf europäischen Staaten und stellen eine Verdrängung von weniger produktiven Unternehmen fest, während die produktivsten Unternehmen auf die Importkonkurrenz mit mehr Innovation reagieren und ihre Marktanteile erfolgreich behaupten oder sogar ausbauen. Die ForscherInnen erklären etwa 15\% des technologischen Wandels im letzten Jahrzehnt mit dem „technologischen Upgrading“ der importkonkurrierenden Unternehmen. Die Expansion der innovativsten und profitabelsten Unternehmen in den Exportbranchen und die Verdrängung wenig produktiver Firmen durch Importkonkurrenz ermöglichen bedeutende Produktivitätssteigerungen und eine günstigere Spezialisierung der heimischen Wirtschaft. Abbildung 35 zeigt das Wachstum bzw. Schrumpfen von unterschiedlich technologieintensiven Unternehmen in Sektoren, die stark bzw. schwach von Importen aus China betroffen sind. Während die Unternehmen mit hoher Technologieintensität unabhängig von der Importkonkurrenz etwa gleich rasch gewachsen sind, hat die Importkonkurrenz für eine raschere Selektion der weniger technologieintensiven Unternehmen in jenen Branchen geführt, in denen die Importe aus China besonders stark gewachsen sind. Die Importkonkurrenz lenkt Arbeit und Kapital rascher von weniger produktiven zu hochproduktiven Unternehmen und trägt zu einem rascheren Produktivitätswachstum bei.

Die von der Importkonkurrenz ausgelöste Reallokation von Ressourcen untersuchen auch Iacovone u.a. (2013) am Beispiel Mexikos. Die Zunahme chinesischer Importe wirkt sehr unterschiedlich auf mexikanische Unternehmen. Große, produktive Firmen wachsen, während die kleinen und weniger profitablen schrumpfen. Der Auswahlprozess findet dabei auch auf der Ebene von Produkten statt. Der Umsatz von marginalen Gütern schrumpft, während der Umsatz der wichtigsten und profitabelsten Produktlinien steigt. Das Importwachstum aus China bedeutet für die mexikanischen Unternehmen nicht schärfere Importkonkurrenz, sondern verbilligt die aus China importierten Vorleistungen, was die Kosten senkt und die Wettbewerbsfähigkeit stärkt. Wieder konnten die großen Unternehmen und die Kernprodukte stärker von der Erweiterung des Beschaffungsmarkts profitieren.

Strukturwandel und Faktorreallokation schaffen unvermeidlich Gewinner und Verlierer, wobei die Verlierer z.B. bei Betriebsschließungen oder beim Niedergang von benachteiligten Regionen meist wesentlich sichtbarer sind. Eine aktive Wettbewerbspolitik, die Zu- und Austritt erleichtert, ist andererseits eine wesentliche Quelle für Produktivitätssteigerungen und Innovation. Eine Verhinderung oder Verschleppung von Insolvenzen durch 
Überbrückungshilfen und andere Subventionen würde den Strukturwandel hemmen und das Neue verhindern. Zwar könnten zunächst Jobs erhalten werden, aber gleichzeitig wird dies mit einer Schwächung von anderen Unternehmen erkauft, die am Markt besser aufgestellt sind. Die Fortführung von unrentablen Firmen in der Hoffnung auf Besserung führt dazu, dass diese den Konkurrenten Marktanteile wegnehmen und damit „still und leise“ die Jobs in Firmen mit besseren Zukunftsperspektiven gefährden. Selbst für die betroffenen ArbeitnehmerInnen mag es letztendlich attraktiver sein, zu Unternehmen mit guten Wachstumsaussichten und damit sicherer Beschäftigung zu wechseln anstatt in den von Insolvenz bedrohten Firmen zu verharren. Indem die weitere Finanzierung gestoppt wird, wird Kapital von unrentablen Verwendungen ferngehalten, damit es in anderen, innovativeren Unternehmen mit höherer Rendite eingesetzt werden kann.

\section{Abbildung 35: Reallokation bei schwacher und starker Importkonkurrenz aus China, 12 EU-Staaten}

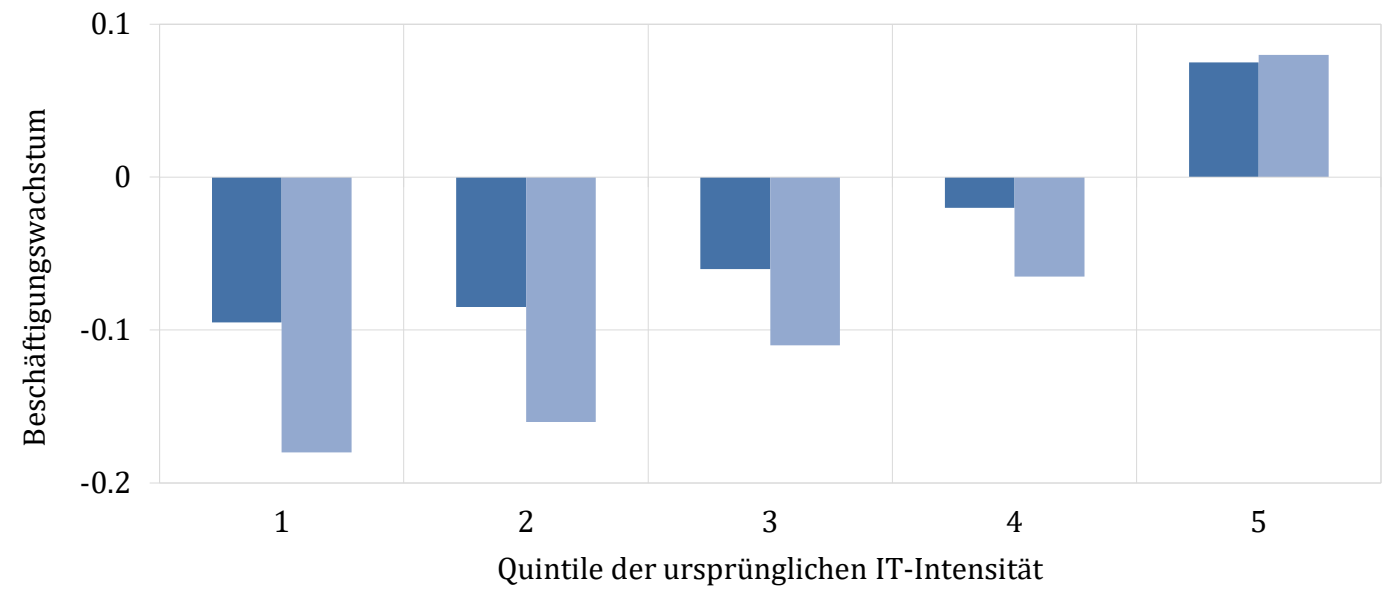

Niedriges Wachstum von chinesischen Importen $\begin{aligned} & \text { Hohes Wachstum von chinesischen Importen } \\ & \text { (Niedrigstes Quintil) }\end{aligned}$
(Höchstes Quintil)

Bemerkung: Beschäftigungswachstum je nach Innovationsgrad der Firmen in Branchen mit schwach und stark steigenden Importen aus China. Die IT-Intensität ist von Quintil 1 zu 5 ansteigend.

Quelle: Bloom u.a. (2011), eigene Darstellung

Innovation und Strukturwandel müssen auch auf den Faktormärkten unterstützt werden. Die ArbeitnehmerInnen setzen sich einem höheren Beschäftigungs- und Einkommensrisiko aus. Sie wollen nicht nur Einkommen, sondern auch wirtschaftliche Sicherheit. Es gehört zu den zentralen Staatsaufgaben, solche Risiken, für die auf dem freien Markt keine adäquate Absicherung möglich ist, über die Sozialversicherung aufzufangen. In einer innovativen Wirtschaft mit raschem technologischem Wandel sind die Arbeitsrisiken größer. Umso mehr braucht es einen gut ausgebauten Sozialstaat. Auf der anderen Seite müssen Strukturwandel und Reallokation von Arbeit und Kapital stattfinden, damit die Innovation sich entfalten und zu mehr Produktivität und Einkommen führen kann. Die Ausgestaltung des Sozialstaats sollte also beides ermöglichen, mehr wirtschaftliche Sicherheit und gleichzeitig raschen Wandel der Arbeit. Am ehesten kann das Modell der „Flexicurity“ diese beiden Ansprüche verbinden und 
auch bei den ArbeitnehmerInnen Akzeptanz für flexible Märkte schaffen (vgl. die Analyse in Davoine und Keuschnigg, 2015, und die dort zitierte Literatur). Nach dem dänischen Vorbild sollte ein Sozialstaat nach dem Flexicurity-Ansatz drei zentrale Elemente verbinden, nämlich (1) Sicherheit, (2) Flexibilität und (3) Aktivierung.

Sicherheit bietet eine Arbeitslosenunterstützung mit angemessenen Ersatzquoten und Dauer der Unterstützung, bevor die ArbeitnehmerInnen auf das niedrigere Niveau der Sozialhilfe bei Langzeitarbeitslosigkeit fallen. Die Höhe der Unterstützung muss zwischen Absicherung des verfügbaren Einkommens und den Anreizen zur aktiven Jobsuche abwägen. Eine Arbeitslosenversicherung ist auch für die Unternehmen wichtig, weil sie die Risikoprämie in den Löhnen, die für riskante Beschäftigung gefordert werden, und damit die Lohnkosten senkt. Nicht zuletzt aufgrund des höheren Risikos sind die Löhne in der Privatwirtschaft höher als in der vergleichsweise sicheren Beschäftigung der Beamten, und sie müssten noch höher sein, wenn es keine oder nur eine rudimentäre Arbeitslosenunterstützung gibt. Flexibilität erfordert einen angemessenen, aber nicht überhöhten Kündigungsschutz, damit die Unternehmen Beschäftigung abbauen können, wenn sie nicht mehr rentabel ist.33 Aktivierung ist das Ziel der aktiven Arbeitsmarktpolitik. Sie unterstützt lebenslanges Lernen sowie Qualifikation und Umschulung während der Arbeitslosigkeit. Damit fördert sie eine schnellere Wiederbeschäftigung in anderen Unternehmen mit besseren Zukunftsaussichten.

Die Länder unterscheiden sich auch sehr stark in ihrer Fähigkeit, Kapital von wenig produktiven Verwendungen abzuziehen und auf innovative wachsende Unternehmen und Branchen hinzulenken (Wurgler, 2000, und Bertrand u.a., 2007). Die Bedeutung des Finanzplatzes Österreich für Innovation und Wachstum streichen Keuschnigg und Kogler (2016) heraus und betonen drei Aspekte: Erstens, je näher ein Land an der Technologiegrenze ist, desto größere und schwierigere Innovationserfolge müssen die Unternehmen erzielen. Die Unternehmen müssen mehr Risiko eingehen und brauchen mehr risikotragendes Beteiligungskapital. Die Kapitalmärkte spielen im Vergleich zu Bankkrediten eine größere Rolle. Zweitens hat im riskantesten Segment der innovativen Firmen Wagniskapital eine wichtige Rolle. Wagniskapital kann auch sehr riskante Firmen noch finanzieren, die keine Bankkredite mehr erhalten würden. Dadurch werden innovative Projekte mit großem Potential möglich, die sonst mangels Finanzierung nicht starten könnten. Zudem ist Wagnisfinanzierung beratungsintensiv und multipliziert das Unternehmenswachstum, so dass Innovationen mehr Wertschöpfung erzielen (siehe Abschnitt 4.5). Drittens braucht es einen gut kapitalisierten Bankensektor, der Kredite in unrentablen Verwendungen fällig stellt und das frei werdende Kapital in Form von neuen Krediten an wachsende Unternehmen vergibt. Die Kreditwürdigkeitsprüfung bei Neuvergabe oder Verlängerung eines Kredits stellt die Tragbarkeit des Kredits fest und wirkt wie eine externe Rentabilitätsprüfung. Banken mit wenig Eigenkapital zögern dagegen oft, faule Kredite fällig zu stellen und Verluste zu realisieren, um ihr schwaches Eigenkapital zu schonen, und verlängern anstatt dessen bestehende Kreditlinien. Umso weniger können sie

33 Angemessen bedeutet eine Abwägung zwischen Flexibilität, um einen Neueinsatz zu ermöglichen, und Stabilität der Beschäftigungsverhältnisse, damit die Unternehmen eher in das Humankapital ihrer Belegschaft investieren und den Ertrag länger nützen können. Ein kumulativer Wissensaufbau ist für innovative Unternehmen ein wichtiger Wettbewerbsvorteil. Dann wäre zu erwarten, dass sie selbst Anstrengungen unternehmen, um die Belegschaft zu kultivieren und an sich zu binden. $\mathrm{Ob}$ Flexibilität oder Stabilität vorteilhafter sind, hängt auch von der Technologie ab (Bartelsman, 2013). 
an neue Unternehmen Kredite vergeben. Banken mit robuster Eigenkapitalausstattung realisieren Verluste früher, schreiben Problemkredite rascher ab und vergeben mehr neue Kredite. Auf diesem Weg unterstützen sie die Kapitalreallokation, Innovation und Wachstum. 


\section{Zentrale Elemente einer neuen Innovationsstrategie}

Österreich hat in den letzten 20 Jahren bei den F\&E-Investitionen bemerkenswert aufgeholt. Beim wichtigsten Indikator, dem Anteil der F\&E-Investitionen am BIP, lag Österreich Mitte der 1990er-Jahre weit hinter den führenden Industriestaaten und hat inzwischen die meisten überholt. Österreich übertrifft beim Anteil F\&E-Investitionen am BIP bereits heute das von der EU für 2020 anvisierte Ziel von drei Prozent. In absoluten Zahlen hat sich das Volumen der F\&E-Investitionen seit 1995 mehr als verdoppelt.

Eine wichtige Rolle in diesem Aufhol- und Überholprozess spielt der industrielle Sektor. Das zeigt sich zunächst bei der F\&E-Tätigkeit des Sektors selbst. Die Betriebe des Verarbeitenden Gewerbes in Österreich geben knapp neun Prozent der Bruttowertschöpfung für F\&EInvestitionen aus. Dieser hohe Anteil sorgt dafür, dass mit 62 Prozent der überwiegende Anteil aller unternehmerischen F\&E-Investitionen auf das Verarbeitende Gewerbe entfällt. Dennoch liegt die F\&E-Intensität des Verarbeitenden Gewerbes deutlich niedriger als in anderen, vergleichbaren EU-Staaten wie Finnland oder Schweden.

Innerhalb der EU zeigt sich eine bemerkenswert hohe Korrelation des Anteils des Verarbeitenden Gewerbes und des Anteils der F\&E-Investitionen am BIP. Es besteht offenbar ein kausaler Zusammenhang zwischen industriellen Aktivitäten und der Fähigkeit zu hohen hohe F\&E-Investitionen. In Österreich hat die Industrie im Vergleich zur restlichen EU zwar einen hohen Stellenwert, aber ihre Forschungsintensität kann noch gesteigert werden. Die heimische Industrie hat nicht nur die Zunahmen der F\&E-Aktivitäten getragen, sondern hat auch das Potenzial zur weiteren Erhöhung und Verbesserung in der Umsetzung.

Auch wenn Österreich bei den F\&E-Investitionen aufholen und sogar überholen konnte, so zeigen sich dennoch in der Umsetzung immer noch Schwächen. Die EU klassifiziert anhand einer Reihe von Indikatoren Dänemark, Deutschland, Finnland, die Niederlande und Schweden als Innovation Leader. Österreich hat es trotz vergleichbarer Werte beim Anteil der Industrie, bei der Produktivität und bei den F\&E-Investitionen bislang allerdings nicht geschafft, in diese Gruppe vorzustoßen. Die der Klassifikation zugrundeliegenden Indikatoren mögen bis zu einem gewissen Grad willkürlich gewählt sein. Dennoch zeigt sich eine gewisse Schwäche bei der Umsetzung von F\&E-Inputs zu Innovationen, also zu neuen Verfahren und Produkten, mit denen die Unternehmen Marktanteile gewinnen können.

Direkte Vergleiche mit den Innovation Leader zeigen deutlich, dass Österreich bei Indikatoren, die die Innovationstätigkeit abbilden, Rückstände aufweist. Beim wichtigsten Indikator, den Patentierungen, sind die führenden Innovationsländer Österreich und dem Rest der EU weit voraus. Auch beim Export wissensintensiver Dienstleistungen sowie dem Anteil innovativer Unternehmen sind Rückstände zu verzeichnen. Dennoch erzielt Österreich ein hohes Produktivitätswachstum und liegt darin vor den meisten Innovation Leader. Insbesondere in den mittleren Technologiesegmenten ist die Produktivität bemerkenswert hoch, was zum sehr guten Abschneiden bei Prozess- und organisatorischen Innovationen passt.

Je weiter ein Land technologisch voranschreitet, umso eher fällt ihm die Rolle zu, selbst die Technologiegrenze zu verschieben, anstatt vorhandene Technologien zu verbessern. Hier zeigt sich eine weitere relative Schwäche. Zwar ist der Anteil der Grundlagenforschung an den gesamten F\&E-Investitionen gestiegen, dennoch liegt dieser Anteil am BIP weit hinter 
führenden Ländern wie Japan oder der Schweiz zurück. Ein Grund ist das Fehlen einer größeren Anzahl von multinationalen Konzernen, die am ehesten in die Grundlagenforschung investieren. Aber auch im universitären Bereich steckt noch viel Verbesserungspotenzial. So hat Österreich durchaus Schwierigkeiten, sowohl hoch qualifizierte ArbeitnehmerInnen und ForscherInnen aus dem Ausland anzulocken als auch das eigenen Spitzenpersonal zu halten.

Die wohl größte Herausforderung Österreichs besteht wohl darin, dass die Unternehmen radikalere Innovationsstrategien verfolgen und neue Produkte und Prozesse mit größerem technologischem Vorsprung entwickeln und zur Marktreife bringen. Diese sind wegen ihres hohen Technologiegehalts nicht einfach zu kopieren und können auf den Weltmärkten eine dominantere Position sichern. Oft sind es gerade Start-Ups, die nicht von den Erfahrungen mit vorhandenen Produkten und Prozessen geprägt sind und eher ganz neue Lösungen ausprobieren. Hier zeigt sich jedoch beim Zugang zu Wagniskapital eine weitere Schwäche. Für Start-Ups ist es hierzulande schwieriger als in anderen Ländern, mit Wagniskapital eine Finanzierung und strategische Beratung aus einer Hand $\mathrm{zu}$ erhalten. Aktive Wagniskapitalgeber drängen ihre Unternehmen zu radikaleren Innovationsstrategien und trimmen sie auf Wachstumskurs. In der Intensivierung der Grundlagenforschung an den Universitäten und der Bereitstellung von Risiko- und Wagniskapital für innovative Unternehmen liegt das größte Potenzial zur weiteren Verbesserung.

Eine neue Innovationsstrategie für den Aufstieg zum Innovation Leader muss die gesamte Wertschöpfungskette der Innovation berücksichtigen, von der staatlichen Grundlagenforschung über die private F\&E bis hin zur Kommerzialisierung in der Produktion. Sie soll eine klare Aufgabenteilung zwischen Staat und Privatwirtschaft ermöglichen, wonach der Staat wichtige Vorleistungen für die private Innovation erbringt und mit fiskalischen und regulatorischen Instrumenten Marktstörungen abbaut, nicht zuletzt damit der Wettbewerb ein besseres Ergebnis erzielt.

Die Innovationsstrategie soll die Heterogenität der Firmen berücksichtigen. Junge innovative Unternehmen leiden häufig an Finanzierungsproblemen wie Mangel an Risikokapital, welcher die rasche Expansion drosselt, und können in der Folge rentable Investitions- und Beschäftigungschancen nicht ausschöpfen. Große Konzerne, die den größten Teil der privaten F\&E-Ausgaben verantworten, haben dagegen wenig Mühe, Finanzierung aufzutreiben. Sie sind an vielen Standorten weltweit präsent und international mobil. Bei ihnen hat die Standortattraktivität Priorität. Damit ist die Politik gefordert, die zunehmende F\&EFörderung im Ausland wie auch andere Standortnachteile wie z.B. den kleinen Binnenmarkt wenigstens teilweise zu kompensieren. Eine Innovationsstrategie soll aber nicht darauf abzielen, die Internationalisierung der Unternehmen zu bremsen, sondern der Abwanderung von F\&E, Produktion und anderen Funktionen über das natürliche Niveau hinaus entgegenzuwirken. Damit trägt das Inland nicht nur die Kosten, sondern sichert sich auch den Ertrag der getätigten F\&E-Investitionen in Form von Einkommen und Beschäftigung.

Die Innovationsstrategie soll einen systemischen Ansatz verfolgen und die Komplementaritäten zwischen den verschiedenen Politikfeldern ausnutzen. Allzu oft wird der Fokus zu eng auf die F\&E-Förderung gelegt. Diese kann jedoch einen kleinen oder großen volkswirtschaftlichen Ertrag haben, je nachdem ob an anderen Stellen Engpässe in der Wertschöpfungskette der Innovation bestehen oder nicht. Wenn es an Innovationsfinanzierung mangelt und die Grundlagenforschung vernachlässigt wird, dann 
kann auch eine noch so großzügige F\&E-Förderung nur beschränkte Wirkung entfalten. Nur wenn ein Politikelement die Wirksamkeit anderer Maßnahmen positiv beeinflusst, also die Elemente komplementär zueinander sind, wird das Ganze mehr als die Summe der Teile. Die folgende Innovationsstrategie in zehn Punkten ist daher als Gesamtkonzept zu verstehen. Angesichts der Zielsetzung der Studie legt sie einen Schwerpunkt auf die Stärkung von F\&Ebetreibenden Unternehmen in Österreich.

(1) Grundlagenforschung ausbauen. Sie erbringt zentrale Vorleistungen für die private Innovation, indem sie F\&E-Personal an der Grenze der Forschung ausbildet und selbst neues, allgemein zugängliches Wissen schafft, das für die Kommerzialisierung verfügbar ist. Neben den Gehältern bestimmen der Zugang zu Forschungsmitteln, die Lehrbelastung und die Finanzierung von forschungsintensiven PhD-Programmen die Wettbewerbsfähigkeit der Grundlagenforschung und forschungsnahen Lehre. Angesichts der Überlastung der Universitäten in der Lehre und ihrer Unterausstattung mit Forschungsmitteln sowie der Budgetknappheit des FWF für die Auswahl und Finanzierung von Forschungsprojekten sind erhebliche öffentliche Mehrausgaben notwendig. Der Ausbau der Grundlagenforschung soll zudem effektive Anreize für universitäre ForscherInnen setzen, mehr Aufmerksamkeit auf mögliche Anwendungen und den Technologietransfer in die Privatwirtschaft richten.34 Die Bedeutung der Grundlagenforschung im Innovationssystem steigt, je mehr sich ein Land der Technologiegrenze annähert und zu einem führenden Innovationsland aufsteigt. Deshalb ist das Quotenziel von 2\% des BIP für den tertiären Bildungssektor eine strategische Zielgröße, die eher früher als später erreicht werden sollte.35 Angesichts der staatlichen Budgetengpässe werden wohl private Mittel als Finanzierungsquelle für F\&E noch wichtiger werden. Es ist zu hinterfragen, ob es bislang tatsächlich gelungen ist, hierfür günstige Rahmenbedingungen wie z.B. ein attraktives Stiftungsrecht in Österreich zu schaffen.

(2) Patentierung unterstützen. Für Erfindungen mit großem wirtschaftlichem Potenzial ist eine Patentierung die vielleicht wichtigste Vorkehrung für einen wirksamen Schutz der Eigentumsrechte. Ein ausgebauter Informationsservice und administrative Unterstützung können die Registrierung eines (Europäischen) Patents erleichtern. Wichtig ist auch, bei Patentstreitigkeiten eine effektive und zügige Rechtsdurchsetzung zu erreichen, damit nicht im Laufe von langen Verfahren neue Rechts- und Investitionsunsicherheiten entstehen.

(3) Gründungsdynamik stärken. Große Konzerne finanzieren zwar den größten Teil der privaten F\&E-Ausgaben, kleine Firmen sind jedoch pro Euro an F\&E-Ausgaben tendenziell effektiver. Junge wagnisfinanzierte Start-Ups haben das größte Wachstumspotenzial, aber auch das größte Risiko. Nur ganz wenige entwickeln sich zu großen Konzernen. Umso wichtiger ist es, viele Firmen zu gründen und mögliche Gründungshemmnisse zu beseitigen. Aktives Unternehmertum braucht eine Wertschätzung der Risikobereitschaft und nicht eine Stigmatisierung des Scheiterns. Das Insolvenzrecht muss zwar einen ausreichenden Gläubigerschutz gewährleisten, sollte aber nicht den Neustart von UnternehmerInnen verhindern. Unter den risikobereiten Personen gibt es SeriengründerInnen, die oft bei der zweiten und dritten Gründung wesentlich erfolgreicher sind, wenn ihnen der Konkurs nach

34 Vgl. die Empfehlungen des RFTE (2016, S. 29) zur Erhöhung der finanziellen Mittel für Grundlagenforschung und Wissenstransfer.

35 Auch die Stärkung der Grundfinanzierung für Universitäten hat der RFTE (2016, S. 24 und S. 49) in seinen Empfehlungen festgehalten. 
dem ersten Versuch noch eine Chance lässt. Entrepreneurship-Trainings an den Universitäten sollen Studierende und junge NachwuchsforscherInnen mit der Option einer Unternehmensgründung als Alternative zur unselbständigen Karriere vertraut machen. Eine weitere Maßnahme zur Steigerung der potenziellen Gründungspopulation wäre ein erleichterter Zugang von gründungswilligen Personen $\mathrm{zu}$ einer Arbeits- und Aufenthaltsbewilligung in Österreich. Bürokratieerleichterungen wie z.B. One-Stop-Shops und E-Government vereinfachen den Schritt in die Selbstständigkeit. 36 Gründerparks schaffen eine günstige Umgebung, in der viele spezialisierte Dienstleister sozusagen in „Rufnähe“ sind und eine Gründung erleichtern. Österreichs Universitäten haben noch Potential, Infrastruktur zu schaffen und zur Verfügung zu stellen, um Spin-offs wie auch Start-ups eine Stütze zu sein. Das Gründungsökosystem an den Universitäten sollte zusätzlich an Stärke gewinnen. 37

(4) Steuerliche Diskriminierung des Risikokapitals abbauen. Unternehmertum erfordert Risikobereitschaft. Bankkredite sind auf Sicherheit aus und beanspruchen die volle Verzinsung und Rückzahlung auch in schwierigen Zeiten. Eigenkapital nimmt dagegen den Unternehmen einen Teil des Risikos ab, indem es an Erträgen und Verlusten gleichermaßen beteiligt ist. Je ehrgeiziger die Innovationen und je größer die Risiken sind, desto mehr sind Unternehmen auf Risikokapital angewiesen. Um die Bereitstellung von Risikokapital nicht zu blockieren, soll die Besteuerung daher einen vollen und unbeschränkten Verlustausgleich und Verlustvortrag auf Investorenseite garantieren und auf Unternehmensebene neben der Abzugsfähigkeit von Fremdkapitalzinsen auch den Steuerabzug von kalkulatorischen Eigenkapitalzinsen zulassen. Damit würde sich auch der Staat gleichmäßig an Gewinnen und Verlusten beteiligen und einen Teil des Risikos mittragen. Eine solche Reform führt keine Steuerbegünstigung ein, sondern beseitigt eine bestehende Diskriminierung. Beide Maßnahmen zusammen würden die Krisenrobustheit der Unternehmen und die Standortattraktivität nachhaltig stärken. Die Reform würde vor allem riskantere und innovativere Unternehmen, die mehr Risikokapital benötigen, überproportional entlasten. Innovative Branchen würden stärker expandieren.

(5) Fiskalische F\&E-Förderung erhalten. Auch bei einer sonst skeptischen Einstellung gegenüber Subventionen gibt es wenigstens drei gute Gründe dafür im Bereich von F\&E: Erstens fließt ein Teil der privaten F\&E-Erträge anderen, nicht zahlenden Nutzern zu. Die fiskalische Förderung soll nach dem Verursacherprinzip diese externen Erträge kompensieren. Zweitens gibt es den strategischen Grund im Systemwettbewerb, nämlich ausländische Förderungen mit einer eigenen F\&E-Begünstigung zu kompensieren und auch den Nachteil des kleinen Binnenmarktes gegenüber großen Ländern auszugleichen.38 Drittens mindert die fiskalische F\&E-Förderung die Steuerlast und baut damit gezielt Finanzierungsschranken dort ab, wo sie am häufigsten vorkommen, nämlich bei jungen innovativen Wachstumsunternehmen. Daher sollte die Forschungsprämie, ein automatischer

36 Österreich ist im E-Government sehr erfolgreich. Nach dem Bericht „Doing Business“ der Weltbank ist jedoch eine Unternehmensgründung immer noch deutlich zeit- und kostenaufwendiger als in vielen vergleichbaren Ländern. Siehe hierzu auch die Empfehlungen des RFTE (2016), S. 40f.

37 Siehe ergänzend die BMWFW-Studie zum universitären Ökosystem von Ecker und Gassler (2016).

38 Fiskalische F\&E-Anreize sind eine sparsame Standortförderung. F\&E-intensive Firmen sind auf dem Weltmarkt tätig und gehören daher zu den mobilsten Unternehmen. Steueranreize für F\&E entlasten gezielt dort, wo die Stärkung der Standortattraktivität am dringendsten ist. Eine Senkung des KÖSt-Steuersatzes entlastet dagegen alle Firmen, ob sie nun mobil sind oder nicht, und ob eine Stärkung der Standortattraktivität notwendig ist oder nicht. 
Steuervorteil für alle F\&E-treibenden Unternehmen, neben den direkten Förderungen (welche Mittel nur bei erfolgreicher Antragstellung vergeben und daher nur ausgewählte Unternehmen unterstützen) generös ausgestaltet bleiben.

(6) Wagnisfinanzierung stärken. Wagniskapitalisten finanzieren hoch riskante Innovationen, wo Banken meist keinen Kredit mehr geben. Sie unterstützen zudem als aktive Investoren das Unternehmenswachstum mit strategischer Beratung und Überwachung. Ein aktiver Wagniskapitalsektor kann sich nur in einem kapitalmarktorientierten Umfeld nachhaltig entwickeln. Priorität sollte daher der Ausbau des privaten Wagniskapitalsektors haben, der langfristig selbsttragend und unabhängig von der aws als öffentlicher Risikokapitalgeber leistungsfähig ist. Wichtig ist, auf eine strikte Wettbewerbsneutralität zwischen öffentlichen und privaten Wagniskapitalgesellschaften zu achten. Angesichts des viel zu kleinen Kapitalmarkts können Wagniskapital und private Beteiligungsgesellschaften in Österreich derzeit kaum genügend Refinanzierung mobilisieren. Daher wird wohl die aws einen Ersatz für die Refinanzierung privater Gesellschaften auf dem Kapitalmarkt bieten müssen. Die aws sollte daher wenigstens vorübergehend das Fund-in-Fund Geschäft stark ausbauen und sich mehr in privaten Beteiligungsgesellschaften engagieren, die ihrerseits die Portfolio-Investments auswählen und betreuen. Alle anderen Maßnahmen zur Stärkung des Kapitalmarktes, wie z.B. die Beseitigung der steuerlichen Diskriminierung des Risikokapitals, betreffen den Wagniskapitalsektor a fortiori.

(7) Kapitalmarkt und Börse stärken. Eigenkapitalfinanzierung ist nicht das Kerngeschäft der Banken, sondern erfolgt vorwiegend über den Kapitalmarkt. Je näher ein Land an der technologischen Grenze ist, desto ehrgeiziger und riskanter müssen die Innovationen werden, und desto wichtiger sind Kapitalmärkte und Börse. Ein Bündel von Reformen ist notwendig, um den Finanzplatz Österreich auf zwei starke Beine zu stellen, nämlich leistungsfähige Banken und liquide Kapitalmärkte. 39 Ein derart großes Anlagevolumen kann jedoch nur von institutionellen Großanlegern kommen. Neben der Beseitigung von steuerlichen Hindernissen, einem starken Kapitalmarktrecht für mehr Rechtssicherheit, um Vertrauen bei den Investoren zu schaffen, und einer Verbesserung des Finanzwissens über gute Anlageprinzipien müssten daher eine Diversifizierung der Altersvorsorge und ein moderater Aufbau einer kapitalgedeckten Säule der Pensionsversicherung erfolgen. Auf allen großen Kapitalmärkten spielen Pensionsfonds eine sehr bedeutsame Rolle.

(8) Märkte offen halten. Wenn der Zugang zu den in- und ausländischen Absatzmärkten versperrt ist, machen Innovationen wenig Sinn. Reibungsloser Marktzugang erleichtert die Kommerzialisierung und stärkt den privaten F\&E-Ertrag. $\mathrm{Zu}$ den Faktoren, die den Marktzugang im Inland erleichtern, zählen ein durchsetzbares Wettbewerbsrecht mit einer klaren Insolvenzordnung, eine weniger kostspielige und maßvolle Regulierung (die bei weitem nicht nur Konsumenten, sondern allzu oft etablierten Anbietern nützen, und vor allem kleine Unternehmen benachteiligen), und eine schnelle und unbürokratische Abwicklung von Gründungen. Auch muss ein Funktionieren der Wettbewerbsbehörde gesichert sein. Gerade in einem kleinen Land müssen innovative Unternehmen rasch die ausländischen Exportmärkte erobern. Der nationale Binnenmarkt ist für die innovativen und hoch spezialisierten Anbieter viel zu klein, um weiter wachsen zu können. Deshalb müssen

39 Siehe Keuschnigg und Kogler (2016) für eine detaillierte Darstellung von Reformen zur Stärkung des Kapitalmarkts. 
die Weiterentwicklung der EU-Mitgliedschaft sowie der Abbau von Handelshemmnissen mit anderen Handelszonen ein wichtiger Bestandteil einer Innovationsstrategie sein.

(9) Reallokation und Strukturwandel erleichtern. Nach der Innovation kommt der Wandel. Die Digitalisierung der Wirtschaft, die Verfügbarkeit von großen Datenmengen und die Automatisierung mit zunehmendem Robotereinsatz auf dem Weg zur Industrie 4.0 beschleunigen den Strukturwandel. Kapital und Arbeit müssen weg von schrumpfenden hin zu innovativen, wachsenden Unternehmen wandern. Die Faktorreallokation macht etwa die Hälfte des Produktivitätswachstums aus. Arbeits- und Kapitalmarktreformen sollen die Reallokation unterstützen und nicht bremsen. In Österreich behindern die Defizite des Kapitalmarktes (geringes Kapitalmarktvolumen, wenige Beteiligungsgesellschaften, wenig Wagniskapital usw.) die Reallokation des Kapitals. Abgesehen von der hohen Steuer- und Abgabenbelastung sind ein moderater Kündigungsschutz und eine ausgebaute aktive Arbeitsmarktpolitik mit dem notwendigen Wandel auf dem Arbeitsmarkt gut vereinbar. Neben den vorgeschlagenen Kapitalmarktreformen ist vor allem aber auch eine Unterstützung der betrieblichen Innovationsprozesse durch die Bildungspolitik essentiell, angefangen von einer Stärkung der IKT-Kompetenzen in den Schulen bis hin zur Umschulung und einem Ausbau der Aus- und Weiterbildung für ältere ArbeitnehmerInnen (lebenslanges Lernen). 40

(10) Innovationsstandort für multinationale Unternehmen sichern. Die Forschungsaktivitäten von multinationalen Unternehmen spielen eine wichtige Rolle für das heimische Innovationssystem. Im Vergleich zu den führenden Innovationsnationen Europas erweist sich Österreich noch als attraktiver Standort für ausländische F\&E-Aktivitäten. Angesichts der steigenden Mobilität von F\&E-Einheiten sowie der abnehmenden Bedeutung Europas als globaler F\&E-Standort wird der Erhalt der Standortattraktivität wichtiger. Die Präsenz von Spitzenuniversitäten wie auch die Verfügbarkeit von hoch qualitativem Humankapitel sind zentrale Standortvorteile für die F\&E-Einheiten multinationaler Unternehmen. Neben den vorgeschlagenen fiskalischen Maßnahmen ist daher eine Stärkung der universitären Ausbildung und der Grundlagenforschung vordringlich. Diese Prioritätensetzung fördert die Standortattraktivität für multinationale Unternehmen, die den produktivsten und innovativsten Teil der Unternehmenslandschaft ausmachen. Sie ist auch eine Voraussetzung, um an die Grenze der technologischen Entwicklung vorzustoßen und damit zum führenden Innovationsland aufzusteigen. Standortvorteile für F\&E-Aktivitäten helfen zudem, den Wandel zur Industrie 4.0 und zur Digitalisierung im globalen Wettbewerb erfolgreich zu meistern. 41

40 Nach der Leitbetriebe Standortstrategie des BMWFW könnte ein Aufbau von Demonstrationsfabriken wichtige Erfahrungen zur besseren Bewältigung des Wandels hin $\mathrm{zu}$ Industrie 4.0 bringen. In Verbindung mit WissenschaftWirtschaftsförderprogrammen wie z.B. COMET können solche Demonstrationsfabriken auch in Schwerpunkte eingebettet werden und dabei gezielt auch Personen der Arbeitswelt miteinbeziehen.

${ }_{41}$ Die Stärke Österreichs bei den Prozessinnovationen ist eine gute Voraussetzung, um die Chancen der Digitalisierung zu nutzen. Da der Einsatz von Industrierobotern vornehmlich bei mittlerer Produktkomplexität rentabel ist, könnte Österreich damit wieder neue komparative Vorteile auch gegenüber osteuropäischen Nachbarländern oder Billiglohnländern wie China gewinnen. Da das Verarbeitende Gewerbe auch in Krisenzeiten die F\&E-Ausgaben aufrechterhält, trägt die Stärkung des industriellen Sektors zu einer stabilen Positionierung als führendes Innovationsland bei. 


\section{Referenzen}

Abramovsky, Laura, Rupert Harrison und Helen Simpson (2007), University Research and the Location of Business R\&D, Economic Journal 117, 114-141.

Acemoglu, Daron (2009), Introduction to Modern Economic Growth, Princeton, Princeton University Press.

Acemoglu, Daron und David Autor (2011), Skills, Tasks and Technologies: Implications for Employment and Earnings, in: Orley Ashenfelter und David Card (Hrsg.), Handbook of Labor Economics 4, Teil B, 1043-1171.

Aghion, Philippe (2016), Competitiveness and Growth Policy Design, in: Christian Keuschnigg (Hrsg.), Moving to the Innovation Frontier, London, Centre for Economic Policy Research, 5-30.

Aghion, Philippe und Peter Howitt (1992), A Model of Growth through Creative Destruction, Econometrica 60, 323-351.

Aghion, Philippe, Nick Bloom, Richard Blundell, Rachel Griffith und Peter Howitt (2005), Competition and Innovation: An Inverted-U Relationship, Quarterly Journal of Economics 120, 701-728.

Aghion, Philippe., Richard Blundell, Rachel Griffith, Peter Howitt und Susanne Prantl (2009), The Effects of Entry on Incumbent Innovation and Productivity, Review of Economics and Statistics 91, 20-32.

Aghion, Phillipe, Julian Boulanger und Elie Cohen (2011), Rethinking Industrial Policy, Bruegel Policy Brief 04.

Agrawal Ajay, Carlos Rosell und Timothy S. Simcoe (2014), Do Tax Credits Affect R\&D Expenditures by Small Firms? Evidence from Canada, NBER WP 20615.

Akcigit, Ufuk, Salomé Baslandze und Stefanie Stantcheva (2015), Taxation and the International Mobility of Inventors, NBER WP 21024.

Alemani, Enrico, Caroline Klein, Isabell Koske, Cristiana Vitale und Isabelle Wanner (2013), New Indicators of Competition Law and Policy in 2013 for OECD and non-OECD Countries, OECD Economics Department WP 1104.

Alstadsaeter, Annette, Salvador Barrios, Gaetan Nicodeme, Agnieszka Maria Skonieczna und Antonio Vezzani (2015), Patent Boxes Design, Patents Location and Local R\&D, European Commission, JCR Technical Reports.

Altomonte, Carlo, Tommaso Aquilante, Gabor Bekes und Gianmarco I.P. Ottaviano (2013), Internationalization and Innovation in Firms: Evidence and Policy, Economic Policy, October, 663-700.

Altomonte, Carlo, Tomasso Aquilante und Gianmarco I.P. Ottaviano (2012), The Triggers of Competitiveness: The EFIGE Cross-Country Report, Bruegel Blueprint No. 17, Brussels.

Altomonte, Carlo, Giorgio Barba Navaretti, Filippo di Mauro und Gianmarco I. P. Ottaviano (2011), Assessing Competitiveness: How Firm-level Data can Help, Policy Contribution 2011/16, Bruegel

Antras, Pol (2005), Incomplete Contracts and the Product Cycle, American Economic Review 95, 1054-1073. 
Arkolakis, Costas, Natalia Ramondo, Andrés Rodríguez-Clare und Stephen Yeaple (2013), Innovation and Production in the Global Economy, NBER WP No. 18972.

Arnold, Jens, Giuseppe Nicoletti und Stefano Scarpetta (2011), Firm Dynamics and Productivity Growth, EIB Papers 16, 90-115.

Arntz, Melanie, Terry Gregory und Ulrich Zierahn (2016), The Risk of Automation for Jobs in OECD Countries: A Comparative Analysis, OECD Social, Employment and Migration Working Papers 189.

Arrow, Kenneth (1962): Economic Welfare and the Allocation of Resources for Invention. In: Universities-National Bureau Committee for Economic Research, Committee on Economic Growth of the Social Science Research Council (Hrsg): The Rate and Direction of Inventive Activity: Economic and Social Factors, 609-410.

AT Kearney (2012), Optimales Wachstum, Wien.

Audretsch, David B., Alex Coad und Agustí Segarra (2014), Innovation and Growth, Small Business Economics 43, 743-749.

Autor, David H. und Michael J. Handel (2013), Putting Tasks to the Test: Human Capital, Job Tasks, and Wages, Journal of Labor Economics 31, 59-96.

Autor, David H., Frank Levy und Richard J. Murnane (2003), The Skill Content of Recent Technological Change: An Empirical Exploration, Quarterly Journal of Economics 118, 1279-1333.

Aw, Bee Yan, Mark. J. Roberts und Daniel Y. Xu (2011), R\&D Investment, Exporting, and Productivity Dynamics. American Economic Review 101, 1312-1344.

Baileya David und De Propris, Lisa (2014) Manufacturing Reshoring and its Limits: the UK Automotive Case, Cambridge J Regions Econ Soc.

Bartelsman, E. (2013), ICT, Reallocation and Productivity. Fellowship Initiative: The Future of EMU, Economic Papers 486.

Bartelsman, Eric, John Haltiwanger und Stefano Scarpetta (2013): Cross-Country Differences in Productivity: The Role of Allocation and Selection, American Economic Review 103, 305334

Becker, Bettina (2015), Public R\&D Policies and Private R\&D Investment: A Survey of the Empirical Evidence, Journal of Economic Surveys 29, 917-942.

Belderbos, Rene, Leo Sleuwaegen, Dieter Somers und Koen De Backer (2016), Where to Locate Innovative Activities in Global Value Chains? Does Co-location Matter? OECD Science, Technology and Industry Policy Paper No. 30, OECD Publishing, Paris.

Berger, Martin, Helmut Gassler und Susanne Meyer (2011), Untersuchung der Kooperationspotentiale österreichischer Unternehmen im Bereich Forschung und Entwicklung außerhalb Europa, Joanneum Research Report 110-2011, Wien.

Bernard, Andrew B., J. Bradford Jensen, Stephen J. Redding und Peter K. Schott (2012), The Empirics of Firm Heterogeneity and International Trade, NBER WP 17627.

Bernard, Andrew B., Valerie Smeets und Frederic Warzynski (2016), Rethinking DeIndustrialization, NBER WP 22114.

Bilir, Kamran L. und Eduardo Morales (2016), The Impact of Innovation in the Multinational Firm, NBER WP 22160. 
Bloom, Nicholas, Mirko Draca und John Van Reenen (2011), Trade Induces Technical Change: The Impact of Chinese Imports in Innovation and Productivity, NBER WP 16717.

BMWF, BMVIT und BMWA (2009), Österreichischer Forschungs- und Technologiebericht 2009, Wien.

BMWF, BMVIT und BMWFJ (2010), Österreichischer Forschungs- und Technologiebericht 2010, Wien.

BMWF, BMVIT und BMWFJ (2013), Österreichischer Forschungs- und Technologiebericht 2013, Wien.

BMWFW (2014a), Universitätsbericht 2014, Wien.

BMWFW (2014b), Leitbetriebe Standortstrategie, Wien.

BMWFW (2016), Wissenschaft in Österreich/Statistiken, Wien.

BMWFW und BMVIT (2014), Österreichischer Forschungs- und Technologiebericht 2014, Wien.

BMWFW und BMVIT (2015), Österreichischer Forschungs- und Technologiebericht 2015, Wien.

BMWFW und BMVIT (2016), Österreichischer Forschungs- und Technologiebericht 2016, Wien.

Böheim, Michael (2010), Wettbewerbspolitik nach der Wirtschaftskrise, WIFOMonatsberichte 10, 831-846.

Boldrin, Michele und David K. Levine (2008), Intellectual Property, in: Steven N. Durlauf und Lawrence E. Blume (Hrsg.), The New Palgrave Dictionary in Economics, 2nd edition, online.

Bradley, Sebastien, Estelle Dauchy und Leslie Robinson (2015), Cross-Country Evidence on the Preliminary Effects of Patent Box Regimes on Patent Activity and Ownership, Tuck School of Business WP No. 2681433.

Brynjolfsson, Erik und Andrew McAfee (2011), Race Against the Machine: How the Digital Revolution is Accelerating Innovation, Driving Productivity, and Irreversibly Transforming Employment and the Economy, Digital Frontier Press, Lexington.

Buccirossi, Paolo (2013), Competition Policy and Productivity Growth: An Empirical Assessment, Review of Economics and Statistics 95, 1324-1336.

Bundesregierung (2011), Der Weg zum Innovation Leader.

Butschek, Felix (2012), Österreichische Wirtschaftsgeschichte, 2. Auflage, Böhlau Verlag, Wien.

Campagna, Mirella Luigia, Attilio Pasetto und Antonio Riti (2012): EFIGE country report: Austria. Brussels.

Capasso, Marco, Tania Treibich und Bart Verspagen (2015), The Medium-term Effect of R\&D on Firm Growth, Small Business Economics 45, 39-62.

Cassiman, Bruno und Elena Golovko (2011), Innovation and Internationalization through Exports, Journal of International Business Studies 42, 56-75.

Chandler, Alfred D. (1977), The Visible Hand, Harvard.

Chiao, Chaoshin (2002), Relationship between Debt, R\&D and Physical Investment: Evidence from US Firm-Level Data, Applied Financial Economics 12, 105-121. 
Christopoulou, Rebekka und Vermeulen, Philip (2012), Markups in the Euro Area over the Period 1981-2004: A Comparison of 50 Sectors, Empirical Economics 42, 53-77.

Coad, Alex (2007), Firm Growth: A Survey, Papers on Economics \& Evolution 0703.

Coad, Alex, Sven-Olov Daunfeldt, Werner Hölzl, Dan Johansson und Paul Nightingale (2014), High-growth Firms: Introduction to the Special Section, Industrial and Corporate Change 23, 91-112.

Colombelli, Alessandra, Jackie Krafft und Francesco Quatraro (2015), Eco-Innovation and Firm Growth: Do Green Gazelles Run Faster? Microeconometric Evidence from a Sample of European Firms, WWW for Europe Working Paper 88.

Colovic, Ana (2010), Towards a Better Understanding of Multinational Enterprises' R\&D Location Choices, in: Farok J. Contractor, Vikas Kumar, Sumit K. Kundu und Torben Pedersen (Hrsg.), Global Outsourcing and Offshoring: An Integrated Approach to Theory and Corporate Strategy, Cambridge, 168-190.

Colovic, Ana und Ulrike Mayrhofer (2011): Optimizing the Location of R\&D and Production Activities: Trends in the Automotive Industry, European Planning Studies 19, 1481-1498.

Culpepper, Pepper D. (2007), Small States and Skill Specificity - Austria, Switzerland, and Interemployer Cleavages in Coordinated Capitalism, Comparative Political Studies 40, 611637.

Czernich, Nina (2014), Forschung und Entwicklung deutscher Unternehmen im Ausland Zielländer: Motive und Schwierigkeiten, Studien zum deutschen Innovationssystem, 132014.

Dachs, Bernhard (2016), Internationalisierung als Aufholprozess im österreichischen Innovationssystem, Wirtschaftspolitische Blätter 2, 427-446.

Dachs, Bernhard und Bernd Ebersberger (2013), The Effects of Production Offshoring on R\&D and Innovation in the Home Country, FIW-Research Reports No. 14.

Dachs, Bernhard, Franziska Kampik, Thomas Scherngell, Georg Zahradnik, Doris HanzlWeiss, Gabor Hunya, Neil Foster, Sandra Leitner, Robert Stehrer und Waltraut Urban (2013), Internationalisation of Business Investments in R\&D and Analysis of their Economic Impact, Innovation Union Competitiveness Papers 2013/1.

Dachs, Bernhard, Martina Dünser und Thomas, Zahradnik (2015), Internationalisation of business investments in R\&D and analysis of their economic impact (BERD Flows), Contract Nr. RTD/DirC/C3/2010/SI2.563818, Brussels.

Da Rin, Marco, Thomas Hellmann und Manju Puri (2013), A Survey of Venture Capital Research, Handbook of the Economics of Finance, 573-648.

Da Rin, Marco, Giovanna Nicodano und Alessandro Sembenelli (2006), Public Policy and the Creation of Active Venture Capital Markets, Journal of Public Economics 90, 1699-1723.

Davoine, Thomas, und Christian Keuschnigg (2015), Flexicurity, Taxes and Job Reallocation, CESifo WP No. 5302.

De Bondt, Raymond und Jan Vandekerckhove (2012), Reflections on the Relation between Competition and Innovation, Journal of Industry, Competition and Trade 12, 7-19. 
Dechezlepretre, Antoine, Elias Einiö, Ralf Martin, Kieu-Trang Nguyen und John Van Reenen (2016), Do Tax Incentives For Research Increase Firm Innovation? An RD Design for R\&D, NBER WP 22405.

Degryse, Christophe (2016), Digitalisation of the Economy and its Impact on Labour Markets, European Trade Union Institute Working Paper 2016.02, Brussels.

Demirel, Pelin und Mariana Mazzucato (2012), Innovation and firm growth: Is R\&D worth it? Industry and Innovation 19(1), 45-62.

Doralt, Werner (2012), Steuerrecht 2012/13, 14. Auflage, Manz Verlag, Wien.

Dunning, J. H. (1998), Location and the Multinational Enterprise: A Neglected Factor? Journal of International Business Studies 29, 45-66.

Ecker, Brigitte und Helmut Gassler (2016), Akademische Spin-offs: Das universitäre Gründungsökosystem in Österreich und der Nutzen von Spin-offs für die Herkunftsuniversität, Studie im Auftrag des BMWFW, Wien.

Egger, Peter und Christian Keuschnigg (2015), Innovation, Trade and Finance, American Economic Journal: Microeconomics 7, 121-157.

Elias, Bernhard und Gerlinde Pöchhacker-Tröscher (2012), Drittmittelfinanzierung universitärer Forschung in Österreich - Entwicklungen, Rahmenbedingungen und Ansatzpunkte zur Stärkung der Drittmittelforschung an österreichischen Universitäten, www.rat-fte.at/publikationen.html\#2012.

Ellram, L. M., Tate, W. L. und Petersen, K. J. (2013), Offshoring and Reshoring: An Update on the Manufacturing Location Decision. Journal of Supply Chain Management 49, 14-22.

Ernst, Christof, Katharina Richter und Nadine Riedel (2014), Corporate Taxation and the Quality of Research and Development, International Tax and Public Finance 21, 694-719.

Esteve-Pérez, Silviano und Diego Rodríguez (2013), The Dynamics of Exports and R\&D in SMEs, Small Business Economics 41, 219-240.

Europäische Kommission (2016), European Innovation Scoreboard 2016, Annex B, Performance per indicator, Brussels.

European Commission (2015), A digital single market strategy for Europe, Communication from the Commission to the European Parliament, the Council, the European Economic and Social Committee and the Committee Of The Regions - COM(2015) 192 final, https://ec.europa.eu/digital-single-market/en/news/digital-single-market-strategyeurope-com2015-192-final.

European Commission (2016), Competition Policy Brief: EU Merger Control and Innovation, Brussels.

EVCA (2015), Annual Report.

Evers, Lisa, Helen Miller und Christoph Spengel (2015), Intellectual Property Box Regimes: Effective Tax Rates and Tax Policy Considerations, International Tax and Public Finance $22,502-530$.

Ewens, Michael und Matthew Rhodes-Kropf (2013), Is a VC Partnership Greater than the Sum of its Partners? NBER WP 19120.

Falck, Oliver, Alexandra Heimisch und Simon Wiederhold (2016), Returns to ICT Skills, Cesifo WP No. 5720. 
Falk, Martin (2014), The Impact of New Goods and Service Products on Firm Growth: Evidence from Austrian Linked Firm-Level Data, Economics of Innovation and New Technology 23, 378-397.

Falk, Martin (2009), Einfluss der Forschungs- und Entwicklungsaktivitäten auf das Unternehmenswachstum in Österreich, WIFO-Monatsberichte 3, 181-194.

Farre-Mensa Joan, Deepak Hegde und Alexander P. Ljungqvist (2016), The Bright Side of Patents, CEPR DP No. 11091.

Ford, Martin (2015), Rise of the Robots: Technology and the Threat of a Jobless Future, Basic Books, New York.

Foster, Lucia, Thomas Haltiwanger und C. J. Krizan (2001), Aggregate Productivity Growth: Lessons from Microeconomics Evidence, in: Charles R. Hulten, Edwin R. Dean and Michael J. Harper (Hrsg.), New developments in Productivity Analysis, Chicago, 303-372.

Fritsch, Michael, Thomas Wein und Hans-Jürgen Ewers (2007), Marktversagen und Wirtschaftspolitik, 7. Auflage, München.

Fritsch, Ursula und Holger Görg (2015), Outsourcing, Importing and Innovation: Evidence from Firm-Level Data for Emerging Economies, Review of International Economics 23, 687714.

Galasso, Alberto und Mark Schankerman (2016), Patent Rights, Innovation and Firm Exit, NBER WP 21769.

Galasso, Alberto und Mark Schankerman (2015), Patent Rights and Innovation by Small and Large Firms, NBER WP 21769.

Garcia-Manjon, Juan Vicente und Enrique Romero-Merino (2012), Research, Development, and Firm Growth: Emprical Evidence From European Top R\&D Spending Firms, Research Policy 41, 1084-1092.

Gassler, Helmut und Brigitte Nones (2008), Internationalisation of R\&D and Embeddedness: the Case of Austria, Journal of Technology Transfer 33, 407-421.

Gersbach, Hans, Ulrich Schetter und Maik T. Schneider (2015), How Much Science? The 5 WS (and 1 H) of Investing in Basic Research, CEPR DP 10482.

Graetz, Georg und Guy Michaels (2015), Robots at Work, CEP DP 1335.

Gray, John. V., Keith Skowronski, Gökce Esenduran und Johnny Rungtusanatham, M. (2013), The Reshoring Phenomenon: What Supply Chain Academics Ought to know and Should Do. Journal of Supply Chain Management 49, 27-33.

Gregory, Terry, Anna Salomons und Ulrich Zierahn (2016), Racing With or Against the Machine? Evidence from Europe, ZEW DP 16-053.

Griffith, Rachel, Rupert Harrison und Helen Simpson (2010), Product Market Reform and Innovation in the EU, Scandinavian Journal of Economics 112, 389-415.

Griffith, Rachel, Helen Miller und Martin O'Connell (2014), Ownership of Intellectual Property and Corporate Taxation, Journal of Public Economics 112, 12-23.

Griffith, Rachel, Stephen Redding und John Van Reenen (2004), Mapping the Two Faces of R\&D: Productivity Growth in a Panel of OECD Countries, Review of Economics and Statistics 86, 883-895. 
Guellec, Dominique und Bruno Van Pottelsberghe (2000), The Impact of Public R\&D Expenditure on Business R\&D, OECD Science, Technology and Industry WP 2000/4.

Håkanson, Lars und Philip Kappen (2016), Live and Let Die: A Survival Analysis of Foreign R\&D Units in Swedish MNEs, International Business Review 25, 1185-1196.

Hall, Brownyn H. (2008), Patents, in: Steven N. Durlauf und Lawrence E. Blume (Hrsg.), The New Palgrave Dictionary in Economics, 2nd edition, online.

Hall, Bronwyn H. und Raffaele Oriani (2006), Does the Market Value R\&D Investment by European Firms? Evidence From a Panel of Manufacturing Firms in France, Germany and Italy, International Journal of Industrial Organization 24, 971-993.

Haltiwanger, John (2011), Firm Dynamics and Productivity Growth, EIB Papers 16, 116-136.

Harrison, Rupert, Jordi Jaumandreu, Jacques Mairesse und Bettina Peters (2008), Does Innovation Stimulate Employment? A Firm-Level Analysis Using Comparable Micro-Data from Four European Countries, NBER WP 14216.

Hellmann, Thomas und Manju Puri (2000), The Interaction Between Product Market and Financing Strategy: The Role of Venture Capital, Review of Financial Studies 13, 959-984.

Hellmann, Thomas, Laura Lindsey und Manju Puri (2008), Building Relationships Early: Banks in Venture Capital, Review of Financial Studies 21, 513-541.

Helpman, Elhanan, Marc J. Melitz und Stephen R. Yeaple (2004), Exports Versus FDI with Heterogeneous Firms, American Economic Review 94, 300-316.

Hochberg, Yael, Calros J. Serrano und Rosemarie H. Ziedonis (2014), Patent Collateral, Investor Committment, and the Market for Venture Lending, NBER WP 20587.

Hölzl, Werner (2011), Unternehmenswachstum im internationalen Vergleich, WIFOMonatsberichte 8, 557-567.

Hölzl, Werner und Lang, Petra (2011), Unternehmensdynamik: Exportstatus und Umsatzproduktivität, WIFO-Monatsberichte 11, 743-754.

Holmes, Thomas und James Schmitz (2010), Competition and Productivity: A Review of the Evidence, Federal Reserve Bank of Minneapolis, Research Department Staff Report 439.

Hvide, Hans K. und Benjamin F. Jones (2016), University Innovation and the Professor's Priviledge, CEPR DP 22057.

Iacovone, Leonardo, Ferdinand Rauch und Alan Winters (2013), Trade as an Engine of Creative Destruction: Mexican Experience with Chinese Competition, Journal of International Economics 89, 379-392.

ISI (2002), Regionale Verteilung von Innovations- und Technologiepotentialen in Deutschland und Europa, Karlsruhe.

IW Köln (Institut der deutschen Wirtschaft Köln, IW Consult) (2015), Digitalisierung, Vernetzung und Strukturwandel: Wege zu mehr Wohlstand, Erster IW-Strukturbericht, Köln.

Jäger, Angela, Cornelius Moll, Oliver Som, Christoph Zanker, Steffen Kinkel und Ralph Lichtner (2015), Analysis of the Impact of Robotic Systems on Employment in the European Union, Studie im Auftrag der Europäischen Kommission, Luxemburg. 
Jaffe, Adam B. (1989), Real Effects of Academic Research, American Economic Review 79, 957970.

Jaffe, Adam B. (1986), Technological Opportunity and Spillovers of R\&D: Evidence from Firms' Patents, Profits, and Market Value, American Economic Review 75, 984-1002.

Jaffe, Adam B. und Trinh Le (2015), The Impact of R\&D Subsidy on Innovation: A Study of New Zealand Firms, NBER WP No. 21479.

Jones, Charles I. und John C. Williams (1998), Measuring the Social Return to R\&D, Quarterly Journal of Economics 113, 1119-1135.

Kanniainen, Vesa und Christian Keuschnigg (2004), Start-up Investment With Scarce Venture Capital Support, Journal of Banking and Finance 28, 1935-1959.

Kaplan, Steven N. und Per Strömberg (2000), Financial Contracting Theory Meets the Real World: An Empirical Study of Venture Capital Contracts, Review of Economic Studies 70, 281-315.

Karkinsky, Tom, und Nadine Riedel (2012), Corporate Taxation and the Choice of Patent Location Within Multinational Firms, Journal of International Economics 88, 176-185.

Keuschnigg, Christian (2016), Unternehmensbesteuerung und Standortattraktivität, Wirtschaftspolitische Blätter 1/2016, 131-153.

Keuschnigg, Christian (2004a), Venture Capital Backed Growth, Journal of Economic Growth 9, 239-261.

Keuschnigg, Christian (2004b), Taxation of a Venture Capitalist with a Portfolio of Firms, Oxford Economic Papers 56, 285-306.

Keuschnigg, Christian und Michael Kogler (2016), Finanzplatz Österreich. Eine Strategie für Wachstum und Stabilität, WPZ Studie, St. Gallen und Wien, www.wpz.com/forschung/studien.

Keuschnigg, Christian und Sardadvar, Sascha (2016), Österreich in der EU oder Öxit?, WPZ Studie, St. Gallen und Wien, www.wpz.com/forschung/studien.

Keuschnigg, Christian, Brigitte Ecker, Helmut Gassler, Helmut Hofer, Sebastian Koch, Hermann Kuschej, Lorenz Lassing, Christian Reiner, Richard Sellner, Edith Skriner und Stefen Vogtenhuber (2014): Vision Österreich 2050. Vorsprung durch Bildung, Forschung und Innovation, Wien.

Klette, Tor J., Jarle Moen und Zvi Griliches (2000), Do Subsidies to Commercial R\&D Reduce Market Failures? Microeconometric Evaluation Studies, Research Policy 29, 471-495.

Kogan, Leonid, Dimitris Papanikolaou, Amit Seru und Noah Stoman (2012), Technological innovation, resource allocation and growth, NBER WP 117769.

Kortum, Sam und Josh Lerner (2000), Assessing the Contribution of Venture Capital to Innovation, Rand Journal of Economics 31, 674-692.

Koske, Isabell, Isabelle Wanner, Rosamaria Bitetti und Omar Barbiero (2015), The 2013 Update of the OECD's Database on Product Market Regulation, OECD Economics Department WP Nr. 1200.

Kromann, Lene, Jan Rose Skaksen und Anders Sorensen (2011), Automation, Labor Productivity and Employment - a Cross Country Comparison, www.aimprojekt.dk/files/robot-employment.pdf. 
Krugman, Paul (1994), Competitiveness: A Dangerous Obsession, in: Paul Krugman (Hrsg.) (1997), Pop Internationalism, The MIT Press, Cambridge [MA] and London.

Lach, Saul (2002), Do R\&D Subsidies Stimulate or Displace Private R\&D? Evidence from Israel, Journal of Industrial Economics 50, 368-390.

Lach, Saul und Mark Schankerman (1989), Dynamics of R\&D and Investment in the Scientific Sector, Journal of Political Economy 97, 880-904.

Leitner, Karl Heinz (2003), Von der Idee zum Markt: Die 50 besten Innovationen Österreichs, Verlag Böhlau, Wien.

Leitner, Karl-Heinz, Bernhard Dachs, Alexander Degelsegger, Brigitte Ecker, Helmut Gassler, Barbara Heller-Schuh, Josef Hochgerner, Jürgen Janger, Dietmar Lampert, Michael Peneder, Michael Ploder, Wolfgang Polt, Thomas Scherngell, Klaus Schuch, Gerhard Streicher, Maximilian Unger, Fabian Unterlass und Georg Zahradnik (2015), Stärkefelder im Innovationssystem: Wissenschaftliche Profilbildung und wirtschaftliche Synergien, AITIS-Report 103, Wien.

Lentz, Rasmus und Dale T. Mortensen (2008), An Empirical Model of Growth Through Product Innovation, Econometrica 76, 1317-1373.

Lerner, Josh (1999), The Government as Venture Capitalist: The Long-Run Impact of the SBIR Program, Journal of Business 72, 285-318.

Lerner, Josh, Norten Sorensen und Per Strömberg (2011), Private Equity and Long-Run Investment: The Case of Innovation, Journal of Finance 66, 445-477.

Lewin, Arie, Silvia Massini und Carine Peeters (2009), Why are Companies Offshoring Innovation? The Emerging Global Race for Talent, Journal of International Business Studies 40, 901-925.

Melitz, Marc J. und Stephen J. Redding (2012), Heterogenous Firms and Trade, NBER WP 18652.

Mettling, Bruno M. (2015), Transformation numérique et vie au travail, Rapport a l'attention de Madame la ministre du Travail, de l'Emploi, de la Formation Professionnelle et du Dialogue Social, http://zevillage.net/wp-content/uploads/2015/09/rapportMettling.pdf.

Miguélez, Ernest und Fink, Carsten (2013), Measuring the International Mobility of Inventors: A New Database, WIPO Economics \& Statistics Series WP 8

Mion, Giordano und Linke Zhu (2013), Import Competition from and Offshoring to China: A Curse or Blessing? Journal of International Economics 89, 202-215.

Mirrlees, James (2011), Tax by Design: The Mirrlees Review, Oxford University Press.

Muûls, Mirabelle und Mauro Pisu (2009) Imports and Exports at the Level of the Firm: Evidence from Belgium, The World Economy 32, 692-734.

Nanda, Ramana, und Matthew Rhodes-Kropf (2016), Regional Variation in Venture Capital: Causes and Consequences, in: Christian Keuschnigg (Hrsg.), Moving to the Innovation Frontier, London, Centre for Economic Policy Research, 55-72,

Nickel, Stephen (1996), Competition and Corporate Performance, Journal of Political Economy 104, 724-746. 
Oberhofer, Harald (2014), Die Wettbewerbsfähigkeit der österreichischen Exportwirtschaft: Die Rolle der KMUs, FIW Policy Brief Nr. 25, Wien.

OECD (2011), Attractiveness for Innovation: Location factors for international investment, OECD Publishing, Paris.

OECD (2015), OECD Innovation Strategy 2015: An Agenda for Policy Action, OECD Publishing, Paris.

OECD (2016), OECD Pensions Outlook 2016, Paris.

Pagani, Laura, Gianluca Argentin, Marco Gui und Luca Stanca (2015), The Impact of Digital Skills on Educational Outcomes: Evidence from Performance Tests, DEMS Working Paper Series No. 304.

Paqué, Karl-Heinz (2009): Die Bilanz. Eine wirtschaftliche Analyse der deutschen Einheit. München.

Peneder, Michael, Julia Bock-Schappelwein, Matthias Firgo, Oliver Fritz und Gerhard Streicher (2016), Österreich im Wandel der Digitalisierung, WIFO-Studie im Auftrag der A1 Telekom Austria AG, Wien.

Peneder, Michael und Gerhard Streicher (2016), De-vs. Re-Industrialisation: Is Structural Change Reversible? www.iioa.org/conferences/23rd/papers/files/1966_20150523051_ Peneder-StreicherIIOA2015.pdf.

Peters, Bettina, Bernhard Dachs, Martina Dünser, Martin Hud, Christian Köhler und Christian Rammer (2014), Firm Growth, Innovation and the Business Cycle, Background Report for the $2014 \quad$ Competitiveness Report, ftp://ftp.zew.de/pub/zewdocs/gutachten/CR2014_BackgroundStudy.pdf.

Pisano, Gary und Shih, Willy (2009), Restoring American Competitiveness, Harvard Business Review July-August, 2-13.

PROGNOS (2015), Digitalisierung als Rahmenbedingung für Wachstum - update, Studie für die vbw, München.

Pöchacker Innovation Consulting und IMAS International (2013), Evaluierung des Förderungsprojektes „Internationale Kommunikationskampagne Forschungsplatz Österreich“, Studie im Auftrag des BMWFJ, Wien.

Poschke, Markus (2010), The Regulation of Entry and Aggregate Productivity Growth, Economic Journal 120, 1175-1200.

Puri, Manju und Rebecca Zarutskie (2012), On the Life-Cycle Dynamics of Venture-Capital and Non-Venture-Capital-Financed Firms, Journal of Finance 67, 2247-2293.

PWC (2013), Unleashing the power of innovation: Global Innovation Survey, Innovation, growth and business strategy, www.pwc.com/gx/en/innovationsurvey/files/innovation_full_report.pdf.

Reiner, Christian (2012), Play it again, Sam: Die Renaissance der Industriepolitik in der Großen Rezession, Wirtschaft und Gesellschaft 38(1), 15-56.

Reinstaller, Andreas (2014), Technologiegeber Österreich. Österreichs Wettbewerbsfähigkeit in Schlüsseltechnologien und Entwicklungspotentiale als Technologiegeber, WIFO, Wien.

Republik Österreich (2011), Der Weg zum Innovation Leader - Strategie der Bundesregierung für Forschung, Technologie und Innovation, Wien. 
RFTE (2014), Finanzierung der Universitäten und der Forschung bis zum Jahr 2020, www.ratfte.at/tl_files/uploads/Presseaussendungen/140127_Pressemappe.pdf.

RFTE (2016), Bericht zur wissenschaftlichen Leistungsfähigkeit Österreichs 2016. Wien.

Rodrik, Dani (2015), Premature Deindustrialization, NBER WP 20935.

Romer, Paul M. (1986), Increasing Returns and Long Run Growth, Journal of Political Economy 94, 1002-1037.

Romer, Paul M. (1990), Endogenous Technological Change, Journal of Political Economy 98, 71-102.

Romer, Paul M. (2007), An Interview with Paul Romer on Economic Growth, http://www.econlib.org/library/Columns/y2007/Romergrowth.html [20.11.2016].

Roubini, Nouriel (2015), Labour in the Digital Age (Part 1 and Part 2), Roubini Global Economics January, https://www.roubini.com/analysis.

Rubine, Loris, Klaus Desmet, Facundo Piguillem und Ananzazu Crespo (2012), Breaking Down the Barriers to Growth in Europe, the fourth EFIGE Policy Report, Breuegel Blueprint Series.

Sachwald, Frédérique (2008): Location Choices within Global Innovation Networks: the Case of Europe, Journal of Technology Transfer 33, 364-378.

Sardadvar, Sascha und Christian Reiner (2016), Does the Presence of High-Skilled Employees Increase Total and High-skilled Employment in the Long Run? Evidence from Austria, Empirica (i.E.)

Schumpeter, Alois (1942), Kapitalismus, Sozialismus und Demokratie, Tübingen.

Schwab, Klaus (2015), The Global Competitiveness Report 2015-2016, Genf, Weltwirtschaftsforum.

Shapiro, Carl (2012): Competition and Innovation. Did Arrow Hit the Bull's Eye? In: Josh Lerner und Scott Stern (Hrsg.): The Rate and Direction of Inventive Activity Revisited, 361410.

Sieber, Susanne (2008), Österreichs Attraktivität für ausländische Direktinvestitionen sowie als Standort für Headquarter-Funktionen, FIW-Research Reports, Nr. 21, Wien.

Siedschlag, Iulia, Donal Smith, Camelia Turcu und Xiaoheng Zhang (2013), What Determines the Location Choice of R\&D Activities by Multinational Firms? Research Policy 42, 14201430.

Sorensen, Morton (2007), How Smart Is Smart Money? A Two-Sided Matching Model of Venture Capital. Journal of Finance 62, 2725-2762.

Stettes, Oliver (2016), Arbeitswelt und Arbeitsmarktordnung der Zukunft: Welche Schlüsse können aus der vorliegenden empirischen Evidenz bereits geschlossen werden? Gutachten der Initiative Neue Soziale Marktwirtschaft, Köln.

Stöllinger, Roman (2015), Eine Anatomie des österreichischen Exportwachstums nach der Krise, FIW Policy Brief 26.

Syverson, Chad (2011), What Determines Productivity? Journal of Economic Literature 49, 326-365. 
Tate, Wendy L. (2014) Offshoring and Reshoring: U.S. Insights and Research Challenges, Journal of Purchasing and Supply Management 20, 66-68

Thursby, Jerry und Marie Thursby (2006), Here or there? A Survey of Factors in Multinational R\&D Location, Washington.

Tichy, Gunther (2015), Wirtschaftsstandort Österreich - von der 'Überholspur' aufs 'Abstellgleis', WIFO-Monatsberichte 88, 635-648

Tomiura, Eiichi (2008), Foreign Outsourcing and the Product Cycle: Evidence from Micro Data, Applied Economics Letters 15, 1019-1022.

Toole, Andrew A. (2012), The Impact of Public Basic Research on Industrial Innovation: Evidence from the Pharmaceutical Industry, Research Policy 41, 1-12.

Tübke, Alexander, Fernando Hervás, Nicola Grassano und Petros Gkotsis (2015), EU R\&D Survey: The 2015 EU Survey on Industrial R\&D Investment Trends, Publications Office of the European Union, Luxembourg.

Türkcan, K. (2014), Exports Margins in Austria's Export Growth. MPRA Paper 53085.

U.S. President's Advisory Panel on Federal Tax Reform (2006), Simple, Fair, and Pro-Growth: Proposals to Fix America's Tax System, Washington, D.C.

Valsamis, Daphné, An de Coen, Valentijn Vanoeteren und Wim Van der Beken (2015), Employment and skills aspects of the digital single market strategy, European Parliament, www.europarl.europa.eu/RegData/etudes/STUD/2015/569967/ IPOL_STU(2015)569967_EN.pdf.

Van Ark, Bart, Mary O'Mahoney und Marcel P. Timmer (2008), The Productivity Gap between Europe and the Unites States: Trends and Causes, Journal of Economic Perspectives 22, 2544.

Veliyath, Rajaram und Rakesh Sambharya (2011), R\&D investment of multinational corporations, Management International Review 51(3), 407-428.

Wallsten, Scott J. (2000), The Effects of Government-industry R\&D Programs on Private R\&D: The Case of the Small Business Innovation Research Program, Rand Journal of Economics 31, 82-100.

World Bank (2016), Doing Business 2016: Measuring Regulatory Quality and Efficiency, World Bank, Washington, DC.

Weyerstraß, Klaus (2016), Analyse der Produktivität Österreichs im internationalen Vergleich, FIW Policy Brief Nr. 31, Wien.

White House (1999), Economic Report to the US-President, Washington, www.presidency.ucsb.edu/economic_reports/1999.pdf.

Wisbauer, Alexander und Regina Fuchs (2014), Außenwanderungen 2012, Statistische Nachrichten Nr. 3, Wien.

Wurgler, Jeffrey (2000), Financial Markets and the Allocation of Capital, Journal of Financial Economics 58, 187-214. 


\section{Abbildungsverzeichnis}

Abbildung 1: Innovation, Unternehmensentwicklung und Standortattraktivität......................

Abbildung 2: F\&E-Quote Österreichs im Vergleich mit den führenden Industriestaaten,

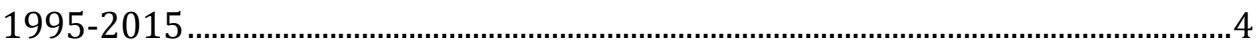

Abbildung 3: F\&E-Intensität des Verarbeitenden Gewerbes, 2009-2013 .................................5

Abbildung 4: Ausgaben für F\&E nach Durchführungssektoren und Forschungsarten, Mio. Euro, 2013

Abbildung 5: Anteile des Verarbeitenden Gewerbes an BWS und F\&E-Ausgaben, EU15Staaten, 2014 ......................................................................................................

Abbildung 6: Akademikerquoten und F\&E-Personal in den EU-Mitgliedstaaten, 2014 .........8

Abbildung 7: Entwicklung der Arbeitsproduktivität, 1998-2015 $(1998=100)$.....................14

Abbildung 8: Arbeitsproduktivität nach Technologieklassen in Tsd. Euro je Beschäftigten,

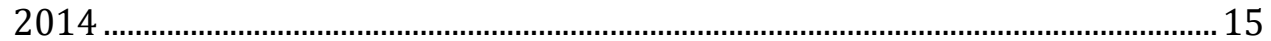

Abbildung 9: Zitierfähige wissenschaftliche Publikationen aus Österreich, 1996-2014 .....17

Abbildung 10: Durchführungs- (links) und Finanzierungssektoren (rechts) der österreichischen F\&E, 2013 .................................................................................... 19

Abbildung 11: Geförderte in \% aller Unternehmen mit Produkt- und/oder

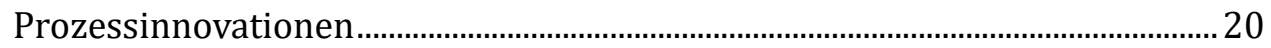

Abbildung 12: Direkte und indirekte F\&E-Fördermaßnahmen in \% des BIP, 2013 .................20

Abbildung 13: Innovation, Unternehmenswachstum und Internationalisierung......................24

Abbildung 14: Anteil Industrie an Bruttowertschöpfung und Arbeitsinput in Stunden, 2015

Abbildung 15: Anteil Industrie (ohne Baugewerbe) an der Bruttowertschöpfung, 1995-2015

26

Abbildung 16: Anteil Industrie an Bruttowertschöpfung und Produktivitätswachstum, EU15Staaten.

Abbildung 17: Bruttoinvestitionen und Bruttoinlandsausgaben für F\&E in \% des BIP, 19952015 33

Abbildung 18: Beschäftigungswachstum, F\&E-Intensität und Unternehmensgröße .............. 35

Abbildung 19: Industrielles Produktionsvolumen nach Technologieniveau (2005=100), Österreich 43

Abbildung 20: Innovation und Beschäftigungswachstum im Konjunkturzyklus, Industrie 1998-2008, in \%.

Abbildung 21: Innovationen und Beschäftigungswachstum im Konjunkturzyklus, 19982008 , in \%

Abbildung 22: Entwicklung der Arbeitsproduktivität, 2010-2015 $(2010=100) \ldots \ldots \ldots \ldots \ldots \ldots . . . . . . . . .49$

Abbildung 23: Investitionsprojekte in Forschung, Entwicklung, Design und Testen (RDDT), 2003-2011

Abbildung 24: Ausländischer Anteil an den privaten F\&E-Ausgaben in \%, 2003 und 2013.54 Abbildung 25: Motive für F\&E-Aktivitäten von deutschen Unternehmen im Ausland, in \% 56 Abbildung 26: Triadische Patentfamilien pro Mio. Einwohner, 2013 ............................................62

Abbildung 27: Grundlagenforschung und wirtschaftliche Entwicklung ......................................65

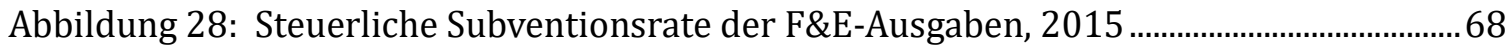

Abbildung 29: Wagniskapital für Unternehmen, 2014 oder letztes verfügbares Jahr.............79 
Abbildung 30: Wagniskapital und Börsenkapitalisierung in Prozent des BIP, 2014 ................82

Abbildung 31: Verwertungsrate in den Konkursverfahren, 2016 ................................................ 88

Abbildung 32: Wettbewerb, Innovation und Strukturwandel .................................................... 89

Abbildung 33: Regulierungsdichte der Produktmärkte (1998-2013)........................................ 92

Abbildung 34: Beiträge zum Produktivitätswachstum der US Industrie, 1977-1997 .............95

Abbildung 35: Reallokation bei schwacher und starker Importkonkurrenz aus China, 12 EU

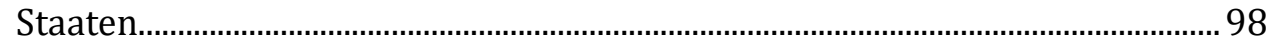




\section{Tabellenverzeichnis}

Tabelle 1: Vergleich Universitäten in Österreich und der Schweiz, 2013 ......................................

Tabelle 2: Produktion, F\&E, Patente im Vergleich, 2014 …........................................................ 13

Tabelle 3: Anteil innovativer Unternehmen (Rang innerhalb der EU in Klammern), 2010-

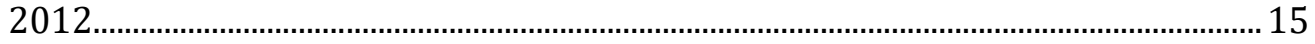

Tabelle 4: Publikationen und Zitierungen, Summen 2010-2014 …..........................................18

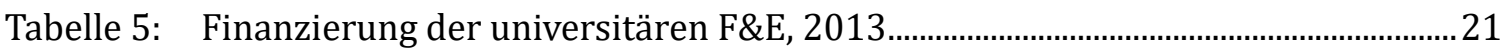

Tabelle 6: Anteil rasch wachsender Unternehmen in Industrie und wissensintensiven

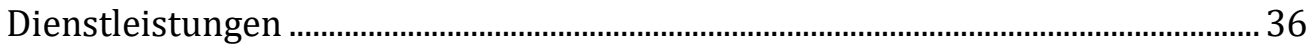

Tabelle 7: Unternehmensgrößen nach Beschäftigung im Industriesektor 2014, Anteile in \%

Tabelle 8: Anteil rasch wachsender Unternehmen in der Industrie ........................................38

Tabelle 9: Beschäftigungsanteile von Wachstumsunternehmen in innovativen Branchen, in \%

Tabelle 10: Wachstumsbarrieren und Unternehmenswachstum, in \% .......................................41

Tabelle 11: Absolute und komparative Handelsvorteile Österreichs, 2014 ...............................50

Tabelle 12: Motive für F\&E-Aktivitäten von deutschen Multis im Ausland (2011) ..................55

Tabelle 13: Patentboxen in Europa, Stand 2014 .......................................................................... 72

Tabelle 14: Wettbewerbspolitische Indikatoren der OECD 2013 im internationalen Vergleich 92

Tabelle 15: Durchschnittliche Gewinnaufschläge 1981-2004 nach Branchen ..........................93 Tabelle 16: Faktorreallokation zwischen innovativen und nicht innovativen Unternehmen94 Tabelle 17: Zerlegung der Umsatzproduktivität für die österreichische Industrie, 2002-2007 


\section{Wirtschaftspolitisches Zentrum WPZ}

Forschung und Kommunikation auf Spitzenniveau für Politik, Wirtschaft und Gesellschaft

Das Wirtschaftspolitische Zentrum (WPZ) ist eine Initiative der Forschungsgemeinschaft für Nationalökonomie (FGN-HSG) an der Universität St. Gallen und ist folgenden Aufgaben gewidmet:

- Spitzenforschung mit Anwendungsbezug

- Wissenstransfer in die wirtschaftspolitische Praxis

- Förderung der wissenschaftlichen Nachwuchstalente

- Information der Öffentlichkeit

Unsere Aktivitäten in der Forschung reichen von wegweisenden Studien in Kooperation mit international führenden Wissenschaftlern bis hin zu fortlaufenden wirtschaftspolitischen Kommentaren. Damit wollen wir die wirtschaftspolitische Diskussion mit grundlegenden Denkanstößen beleben und eine konsequente Reformagenda für Österreich entwickeln, um die großen Herausforderungen besser zu lösen. Die Erkenntnisse und Ergebnisse der modernen Theorie und empirischen Forschung sollen zugänglich aufbereitet und kommuniziert werden, damit sie von Entscheidungsträgern und der Öffentlichkeit wahrgenommen und genutzt werden können und für die politische Entscheidungsfindung Relevanz entwickeln.

Wir freuen uns, wenn Sie unsere Initiativen unterstützen und das WPZ weiterempfehlen. Informieren Sie sich auf www.wpz-fgn.com über unsere Aktivitäten und kontaktieren Sie uns unter office@wpz-fgn.com.

Wirtschaftspolitisches Zentrum | www.wpz-fgn.com | office@wpz-fgn.com 